\title{
All roads lead to thrombus formation : demystifying platelet signaling pathways
}

Citation for published version (APA):

Gilio, K. (2012). All roads lead to thrombus formation : demystifying platelet signaling pathways. [Doctoral Thesis, Maastricht University]. Maastricht University. https://doi.org/10.26481/dis.20121112kg

Document status and date:

Published: 01/01/2012

DOI:

$10.26481 /$ dis.20121112kg

Document Version:

Publisher's PDF, also known as Version of record

\section{Please check the document version of this publication:}

- A submitted manuscript is the version of the article upon submission and before peer-review. There can be important differences between the submitted version and the official published version of record.

People interested in the research are advised to contact the author for the final version of the publication, or visit the DOI to the publisher's website.

- The final author version and the galley proof are versions of the publication after peer review.

- The final published version features the final layout of the paper including the volume, issue and page numbers.

Link to publication

\footnotetext{
General rights rights.

- You may freely distribute the URL identifying the publication in the public portal. please follow below link for the End User Agreement:

www.umlib.nl/taverne-license

Take down policy

If you believe that this document breaches copyright please contact us at:

repository@maastrichtuniversity.nl

providing details and we will investigate your claim.
}

Copyright and moral rights for the publications made accessible in the public portal are retained by the authors and/or other copyright owners and it is a condition of accessing publications that users recognise and abide by the legal requirements associated with these

- Users may download and print one copy of any publication from the public portal for the purpose of private study or research.

- You may not further distribute the material or use it for any profit-making activity or commercial gain

If the publication is distributed under the terms of Article $25 \mathrm{fa}$ of the Dutch Copyright Act, indicated by the "Taverne" license above, 


\section{ALLROADSLEADTO THROMBUSFORMATION}

Demystifying platelet signaling pathways 
ALL ROADS LEAD TO THROMBUS FORMATION

Demystifying platelet signaling pathways

Thesis Universiteit Maastricht

ISBN 978-90-902715-0-7

(c) Karen Gilio, Maastricht 2012

Printed by Drukkerij Gijsemberg

Cover layout by Emile Voeten 


\title{
ALLROADSLEADTO THROMBUSFORMATION
}

Demystifying platelet signaling pathways

\author{
PROEFSCHRIFT
}

ter verkrijging van de graad van doctor aan de Universiteit Maastricht, op gezag van de Rector Magnificus,

Prof. dr. L.L.G. Soete, volgens het besluit van het College van Decanen, in het openbaar te verdedigen op

maandag 12 november 2012 om 14.00 uur

door

Karen Gilio

Geboren op 14 juli 1983 te Maaseik, België 


\section{Promotor}

Prof. Dr. J. W. M. Heemskerk

Department of Biochemistry, CARIM, Maastricht University

\section{Copromotor}

Dr. J. M. E. M. Cosemans

Department of Biochemistry, CARIM, Maastricht University

\section{Beoordelingscommissie}

Prof. Dr. J. G. R. De Mey (voorzitter)

Prof. Dr. H. ten Cate

Prof. Dr. M. G. A. oude Egbrink

Dr. R. Nieuwland (Academisch Medisch Centrum Amsterdam)

Prof. Dr. A. W. Poole (School of Medical Sciences, Bristol, UK)

Financial support by the Netherlands Heart Foundation for the publication of this thesis is gratefully acknowledged.

Additional financial support for the publication of this thesis by Pfizer, ABN AMRO, and Emfret Analytics is acknowledged.

Additional financial support for the publication of this thesis by Stichting Hartsvrienden RESCAR is gratefully acknowledged. 
Nil volentibus arduum

Voor Willem 



\section{Contents}

Chapter $1 \quad$ General introduction 9

Chapter 2 Collagen-mimetic peptides mediate flow-dependent thrombus formation by high- or low-affinity binding of integrin $\alpha_{2} \beta_{1}$ and glycoprotein VI

Chapter 3 Non-redundant roles of phosphoinositide 3-kinase isoforms $\alpha$ and $\beta$ in glycoprotein $\mathrm{VI}$-induced platelet signaling and thrombus formation

Chapter $4 \quad$ PKC $\alpha$ regulates platelet granule secretion and thrombus formation 65 in mice

Chapter 5 Functional divergence of platelet protein kinase $\mathrm{C}$ isoforms in thrombus formation on collagen

Chapter 6 Roles of platelet STIM1 and Orai1 in glycoprotein VI- and thrombindependent procoagulant activity and thrombus formation

Chapter 7 Regulation of integrin $\alpha$ llb $\beta_{3}$ closure in phosphatidylserineexposing platelets

Chapter 8 General discussion

Summary

Samenvatting 165

Dankwoord

Curriculum Vitae

Publications

Addendum 



\section{Chapter 1}

General introduction 
Hemostasis assures the cessation of bleeding at sites of vessel injury, while thrombosis is the pathophysiological process of uncontrolled thrombus formation, potentially developing into vessel occlusion and subsequent heart attack or stroke. The cellular key role in these processes is carried out by platelets. These 1-3 $\mu \mathrm{m}$ discoid anuclear blood cell elements are shed from megakaryocytes in the bone marrow, and circulate in the human blood at concentrations of $150-400 \times 10^{9} / \mathrm{L}$ during 7-10 days, after which they are cleared by the spleen, liver and bone marrow (1). This introductory chapter gives a general overview of key intracellular signaling processes and platelet activation in thrombus formation that are relevant for this thesis.

\section{Platelet activation, aggregation and procoagulant activity in thrombus formation}

Platelets are kept in a resting state because of their continuous encounter with vessel wall derived paracrine molecules like prostacyclin and nitric oxide. Upon vessel wall injury, exposed subendothelial extracellular matrix components, particularly collagen, together with von Willebrand factor (VWF), derived from endothelial cells and plasma (2), provide the main triggers for platelet adhesion and subsequent platelet aggregation and thrombus formation (Figure 1). Under arterial blood flow conditions, platelet adhesion and activation are mediated by the VWF receptor glycoprotein (GP)Ib-V-IX $(3,4)$, and the collagen receptors, integrin $\alpha_{2} \beta_{1}$ and immunoglobulin-like receptor GPVI $(5,6)$. In the context of this thesis, thrombus formation is defined as the overall result of platelet activation, aggregation, and procoagulant activity during whole blood flow over a thrombogenic surface in vitro or in vivo (Figure 1).

An essential step in platelet aggregation is activation of the integrin $\alpha$ llb $\beta_{3}$. In stimulated platelets, this abundantly expressed integrin changes in conformational state, in which its affinity increases for fibrinogen and vWF (7-10). By forming intercellular bridges, fibrinogen, and under high shear conditions also vWF, mediate platelet-platelet interactions. Interestingly, the activation of integrin $\alpha$ llb $\beta_{3}$ is a reversible process, and thus its continuous activation is required for stable thrombus formation (10).

Activated platelets release the contents of two types of secretory granules, namely dense granules (containing e.g. ADP, ATP, polyphosphates and $\mathrm{Ca}^{2+}$ ) and alpha-granules (containing multiple proteins, among which membrane-bound P-selectin, VWF, fibrinogen and coagulation factor $V$ ). Secretion of the granule contents by collagen-adhered platelets is an important step in the recruitment of platelets from flowing blood. In addition, activated platelets produce thromboxane $A_{2}$ from arachidonic acid released from the membrane phospholipids by phospholipase A2. These platelet-derived secondary mediators bind to signaling receptors on nearby platelets, i.e. thromboxane $A_{2}$ to the TP receptor (11), ADP to the $P_{2} Y_{1}$ and $P_{2} Y_{12}$ receptors (12), and ATP to the $P_{2} X_{1}$ receptor (13). 


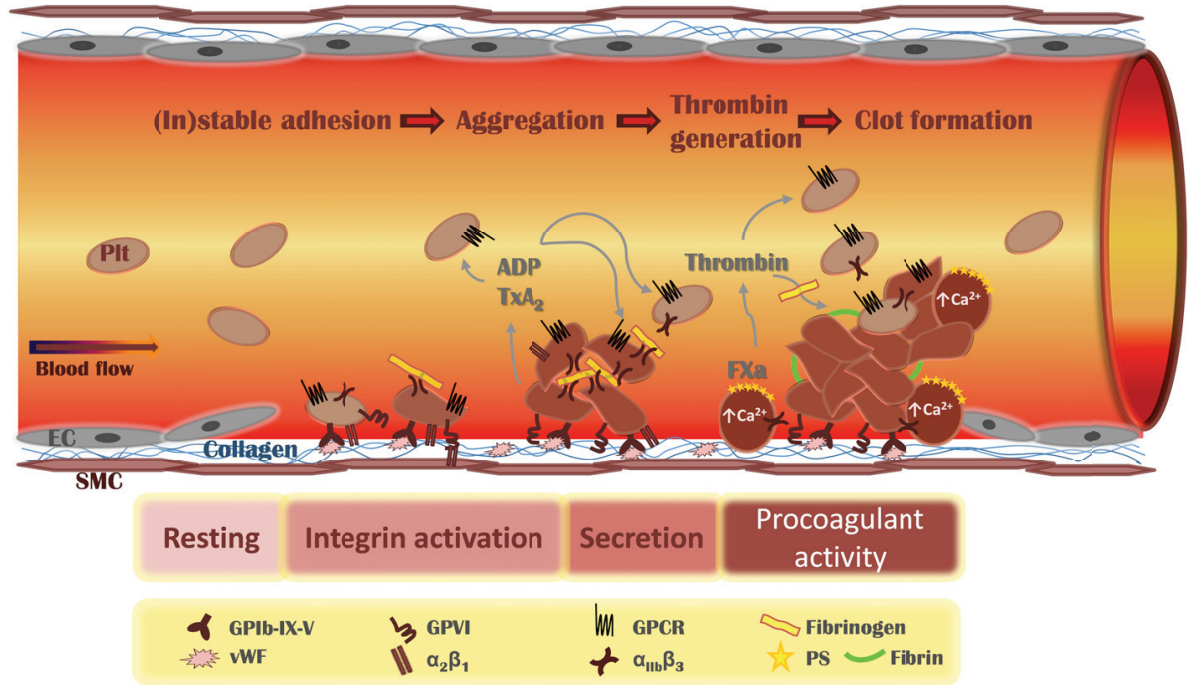

Figure 1. Schematic overview of platelet activation, aggregation and procoagulant activity in arterial thrombus formation. Collagen fibers exposed upon vascular damage form a thrombogenic surface for vWF binding, platelet adhesion and subsequent platelet integrin activation, secretion, aggregation, thrombin generation and fibrin clot formation. Initially, platelets transiently interact with collagen-bound vWF via GPIb-IX-V. They stably adhere and become activated via interaction with the collagen receptors, integrin $\alpha_{2} \beta_{1}$ and GPVI. This activation triggers integrin $\alpha_{\text {llb }} \beta_{3}$ to undergo a conformational transition into a high-affinity ligand binding state. The platelets aggregate via fibrinogen binding to the activated $\alpha \mathrm{llb} \beta_{3}$, thereby forming a platelet thrombus. Concomitant release of autocrine mediators, like ADP and thromboxane $A 2$, results in the recruitment of additional platelets to the growing thrombus. A subpopulation of platelets, e.g. directly in contact with collagen, has a balloonlike shape and sustained high cytosolic $\mathrm{Ca}^{2+}$ levels. These platelets expose negatively charged PS at their outer membrane, and then become procoagulant. The PS serves as a catalytic surface for generation of activated factor $\mathrm{Xa}(\mathrm{FXa})$ and thrombin. The formed thrombin consolidates the thrombus, e.g. by conversion of fibrinogen into fibrin and by acting as a strong platelet agonist. Abbreviations: EC, endothelial cells; GPCR, G-protein-coupled receptor; Plt, platelet; SMC, smooth muscle cells.

Upon thrombus formation, a subpopulation of the platelets in the thrombus typically displays a high cytosolic $\mathrm{Ca}^{2+}$ concentration (14). These platelets are characterized by the expression of negatively charged phosphatidylserine (PS) at their outer surface. Earlier work has shown that segregation of platelet aggregatory and procoagulant microdomains in thrombus formation is regulated by transient activation of integrin $\alpha$ llb $\beta_{3}$. Thrombin, generated by factors $\mathrm{Xa}$ and $\mathrm{Va}$ at the surface of procoagulant platelets, is a central connecting component between the activation of platelets and the coagulation system. Thrombin contributes to the coagulation process by activating other coagulation factors and by converting fibrinogen into fibrin, which consolidates the thrombus. In addition, thrombin acts as a potent platelet agonist by proteolytically activating the G-protein coupled, proteaseactivated receptors, PAR1 and PAR4 (15). Only very little is known yet on the causes, intracellular signaling processes and consequences of this heterogeneity in thrombus formation, although it is likely that thrombin can play an important role herein. 


\section{Collagen-based substrates for adhesive platelet receptors}

The fibril-forming collagens type I and III are among the most potent platelet-activating components of the vascular sub-endothelium and atherosclerotic plaques $(3,16)$. Platelet interaction to either collagen type involves GPVI and integrin $\alpha_{2} \beta_{1}$ receptors. While there is no doubt on the function of GPVI as the key platelet-activating collagen receptor, it is still controversial whether also integrin $\alpha_{2} \beta_{1}$ has a signaling role in collagen-induced platelet activation $(17,18)$. GPVI binds to and is activated by $(G P O)_{n}$ sequences within the triplehelical collagen chains $(19,20)$. In contrast, integrin $\alpha_{2} \beta_{1}$ recognizes collagen motifs with a GXX'GER sequence, which are partly different for collagen type I (GFOGER, GMOGER, GLOGER and GASGER) and collagen type III (GMOGER, GLSGER and GAOGER) (21-23). Under static conditions, triple-helical collagen-like peptides containing such GXX'GER motifs indeed bind integrin $\alpha_{2} \beta_{1}$, albeit with different affinities $(20,22)$. The variation of integrin-binding motifs has made it difficult to precisely dissect the function of integrin $\alpha_{2} \beta_{1}$ in platelet-collagen interactions. We reasoned that flow perfusion studies are needed, using the collagen-mimetic peptides with various integrin-binding motifs, to better understand the role of integrin $\alpha_{2} \beta_{1}$ in thrombus formation.

\section{Platelet signaling through phosphoinositide 3-kinase isoforms}

Human and mice platelets contain four different phosphoinositide 3-kinase ( $\mathrm{Pl}_{3} \mathrm{~K}$ ) isoforms, distinguished according to their catalytic subunit, e.g. $\mathrm{p} 110 \alpha, \mathrm{p} 110 \beta$ and $\mathrm{p} 110 \delta$ (class IA isoforms) and p110y (class IB isoform) (24). The class IA and IB isoforms use separate sets of regulatory subunits. For class $I A$, these are the subunits indicated as $p 85 \alpha / \beta, p 55 \alpha / \gamma$ and P50 $\alpha$, whereas for class IB the regulatory subunit is p101Y (Figure 2A). Below, the isoforms will be named according to their catalytic subunit, i.e. $\mathrm{Pl}_{3} \mathrm{~K} \alpha, \beta, \gamma$ and $\delta$.

Platelet activation via GPVI depends on phosphorylation of the co-receptor, Fc receptor $\gamma$-chain (25), caused by the Src family kinases, Fyn and Lyn (26). Once phosphorylated, the Fc receptor $\gamma$-chain binds the tyrosine kinase Syk via its immunoreceptor tyrosine-based activation motif (ITAM) (27). This initiates a Syk-dependent signaling cascade, leading to formation of a signalosome around the transmembrane linker for activation of T cells (LAT), which serves as a docking site for other signaling proteins, including $\mathrm{Pl}_{3} \mathrm{~K}$ isoforms and phospholipase $C_{\gamma 2}(28,29)$. This is briefly schematized in Figure $2 \mathrm{~B}$.

For GPVI-stimulated platelets, the general concept is that $\mathrm{SH}_{2}$ domain-containing $\mathrm{Pl}_{3} \mathrm{~K}$ regulatory subunits bind to the LAT signalosome, and recruit the corresponding catalytic subunits to the plasma membrane. This may lead to a positive feedback signal, since $\mathrm{Pl}_{3} \mathrm{~K}$ contributes to phospholipase $\mathrm{C}_{2} \gamma$ activation downstream of GPVI activation (28). Other agonists that evoke recruitment of $\mathrm{Pl}_{3} \mathrm{~K}$ isoforms to the plasma membrane are ADP via the $\mathrm{P}_{2} \mathrm{Y}_{12}$ receptor, and thrombin via the $\mathrm{PAR}_{1} / 4$ receptors. 
Class IA

\begin{tabular}{|c|c|c|c|c|c|c|c|}
\hline \multicolumn{4}{|r|}{$\mathrm{N}$} & ADr & \multicolumn{2}{|c|}{ Ras-B } & $\mathrm{C} 2$ \\
\hline $\mathrm{SH} 3$ & $\mathrm{Pr}$ & $\mathrm{BH}$ & $\mathrm{Pr}$ & $\mathrm{SH} 2$ & Int-5H2 & $\mathrm{SH} 2$ & $p 85 \alpha, \beta$ \\
\hline & & & $\mathrm{Pr}$ & $\mathrm{SH} 2$ & Int-SH2 & $\mathrm{SH}_{2}$ & $p 55 \alpha, \gamma$ \\
\hline & & & Pr & $\mathrm{SH} 2$ & Int-SH2 & $\mathrm{SH} 2$ & $p 50 \alpha$ \\
\hline
\end{tabular}

C $p 110 \alpha, \beta, \delta$

$\mathrm{N}$ ADr $\quad$ Ras-B

\section{C2 101}

B

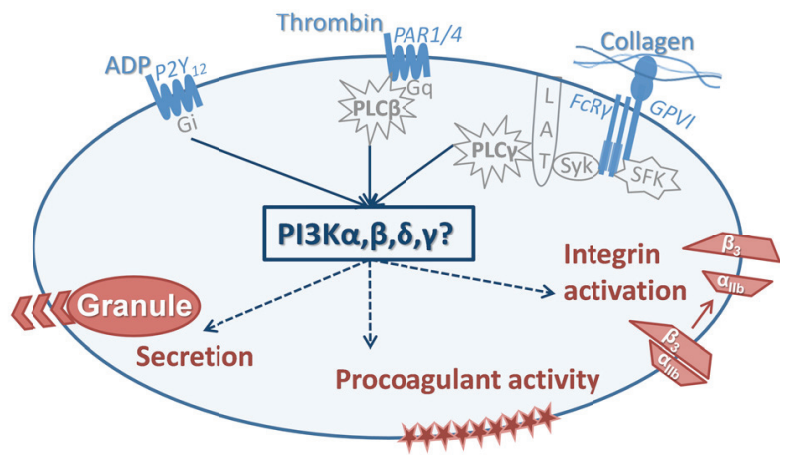

Figure 2. Domain structure and functions of phosphoinositide 3-kinases in platelet activation. $A_{\text {, }}$ Structure of class IA and IB $\mathrm{Pl}_{3} \mathrm{~K}$ isoforms, expressed in mouse and human platelets. Isoforms of both classes contain separate catalytic and regulatory subunits. In class IA isoforms, the inter-SH2 (Int-SH2) domains of the regulatory subunits $\mathrm{p} 85 \alpha / \beta, \mathrm{p} 55 \alpha / \gamma$ or $\mathrm{p}_{50} \alpha$ interact with $\mathrm{N}$-terminal domains of the catalytic subunits, $\mathrm{p} 110 \alpha, \beta$, or $\delta$. Subsequent binding of the $\mathrm{SH}_{2}$ domains with the adaptor region (ADr) in the catalytic subunit is a prerequisite for $\mathrm{Pl}_{3} \mathrm{~K}$ activity. The class IB $\mathrm{p}_{101}$ regulatory subunit contains neither Int-SH2 nor $\mathrm{SH}_{2}$ domains, and specifically interacts with the N-terminal of the p110y catalytic subunit. $\mathrm{B}$, Agonist-induced activation of unspecified $\mathrm{Pl}_{3} \mathrm{~K}$ isoforms can be triggered by: ADPreceptor $\mathrm{P}_{2} \mathrm{Y}_{12}$ stimulation via the GTP-binding protein $\mathrm{Gi}$; thrombin-receptor PAR1/4 stimulation via $\mathrm{Gq}$ and phospholipase $\mathrm{C} \beta(\mathrm{PLC} \beta)$; or by collagen-receptor GPVI stimulation via the LAT signalosome (involving the protein kinase Syk and Src-family kinases (SFK)) and PLC 2 2. The Pl3K isoforms are considered to stimulate platelet secretion, integrin activation, and procoagulant activity. Which $\mathrm{Pl}_{3} \mathrm{~K}$ isoforms are involved in these key platelet responses and which isoform exactly becomes activated by which platelet agonist remains to be elucidated. Abbreviations: $\mathrm{BH}, \mathrm{Bcr}$ homology domain; $\mathrm{C}_{2}$, phospholipid binding domain; CATr, catalytic domain; PIK, helical phosphatidylinositol kinase homology

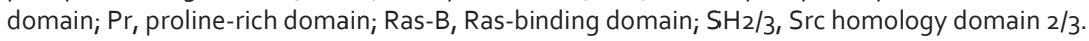

Class IA and IB PI3K isoforms act by phosphorylating the 3-hydroxyl position of phosphoinositides, in particular phosphatidylinositol 4,5-bisphosphate, generating phosphatidylinositol 3,4,5-trisphosphate (30). Interestingly, they compete herein with phospholipase C isoforms, which catalyze the hydrolysis of phosphatidylinositol 4,5-biphosphate into inositol 1,4,5-trisphosphate (IP3) and diacylglycerol. With respect to platelets, there is limited evidence that individual class I $\mathrm{Pl}_{3} \mathrm{~K}$ isoforms have distinct cellular functions. For instance, $\mathrm{P}_{2} \mathrm{Y}_{12}$ stimulation with $\mathrm{ADP}$ activates only the $\mathrm{p}_{110} \beta\left(\mathrm{Pl}_{3} \mathrm{~K} \beta\right)$ and $\mathrm{p}_{110} \mathrm{O}\left(\mathrm{Pl}_{3} \mathrm{~K} \gamma\right)$ isoforms, which contribute to integrin $\alpha$ llb $\beta_{3}$ activation and platelet aggregation (31-34). It is still debated which of the $\mathrm{PI}_{3} \mathrm{~K}$ isoforms are directly activated through the GPVI signaling path- 
ways, and which isoforms are activated indirectly, e.g. following ADP/P2 $Y_{12}$ receptor stimulation.

Also unclear are the downstream signaling events evoked by the various $\mathrm{Pl}_{3} \mathrm{~K}$ isoforms in platelets. A possible, though poorly explored, target of $\mathrm{Pl}_{3} \mathrm{~K}$ is the small GTPase Rapib, which is considered to play a major role in $\alpha \mathrm{llb} \beta 3$ activation. Platelet agonists such as ADP and thrombin produce the active GTP-bound form of Rap 1 b, partly in a $\mathrm{Pl}_{3} \mathrm{~K} \beta$-dependent manner $(35,36)$. Whether and how Rap1b is activated via GPVI or $\mathrm{P}_{2} \mathrm{Y}_{12}$, and which (other) $\mathrm{Pl}_{3} \mathrm{~K}$ isoforms are involved have remained vague. Given their central place in the platelet signaling network, $\mathrm{Pl}_{3} \mathrm{~K}$ isoforms likely play a role in granule secretion and procoagulant activity (Figure 2B). Again, precisely how is unclear. We addressed these issues by a combined pharmacological ( $\mathrm{Pl}_{3} \mathrm{~K}$ isoforms specifice inhibitors) and genetic approach. For the latter the only relevant mice available were deficient in $\mathrm{p} 85 \alpha$, which is the main regulatory subunit of class IA isoforms, or in p110y, being the catalytic subunit of $\mathrm{Pl}_{3} \mathrm{~K} \gamma$.

\section{Platelet signaling through protein kinases $\mathrm{C}$ isoforms}

The protein kinase $C$ (PKC) family of serine-threonine kinases comprises multiple, abundantly expressed members. These PKC isoforms are grouped into three classes (37): conventional isoforms $(\alpha, \beta \mathrm{I}, \beta \mathrm{II}, \gamma)$, which are activated by $\mathrm{Ca}^{2+} /$ diacylglycerol; novel isoforms $(\delta, \varepsilon, \eta, \theta)$, activated by diacylglycerol alone; and atypical isoforms $(\zeta, 1 / \lambda)$, which are diacylglycerol-independent (Figure $3 \mathrm{~A}$ ). All isoforms consist of one protein chain, containing both a catalytic and regulatory subunit. Human and mouse platelets highly express the conventional PKC isoforms $\alpha$ and $\beta$, and the novel isoforms $\delta$ and $\theta$ (38-42). Not much is known about atypical PKC isoforms in platelets.

Pharmacological studies have demonstrated an important positive role for PKC activity in key platelet responses, such as integrin $\alpha$ llb $\beta_{3}$ activation, secretion and aggregation $(43-46)$. On the other hand, there is evidence for a negative regulatory role of PKC in other processes, e.g. lowering of the $\mathrm{Ca}^{2+}$ responses by promotion of $\mathrm{Ca}^{2+}$ extrusion $(47,48)$, and suppression of platelet procoagulant activity. This raises the question whether perhaps the conventional and novel PKC isoforms may have distinct or even opposing roles in the general control of platelet activation (49). This is addressed in Figure $3 \mathrm{~B}$.

At the start of this thesis, only little was known about functions of individual PKC isoforms in platelets. Using biochemical approaches, $\mathrm{PKC} \alpha$ had been identified as a stimulator of $\alpha$-granule and dense-granule secretion (50) as well as platelet aggregation (51). With biochemical and pharmacological approaches, it was shown that PKC $\alpha$ interacts with two tyrosine kinases, Syk and Src, to regulate their activity (52). PKC $\beta$ was reported to be a positive regulator of outside-in $\alpha$ llb $\beta_{3}$ signaling in platelets (53). In contrast, there was uncertainty about the functions of the novel PKC isoforms, PKC $\delta$ and PKC $\theta$, because for both either positive or negative signaling functions have been reported (54-57). 
A

\begin{tabular}{|c|c|c|c|c|c|}
\hline \multirow[b]{2}{*}{ CPKC } & \multicolumn{2}{|c|}{ Regulatory } & Hinge & Catalytic region & \multirow[b]{2}{*}{ C $P K C \alpha, \beta, \gamma$} \\
\hline & DAG & & ATP & Substrate & \\
\hline$K C \mathrm{~N}$ & $\mathrm{Ca}^{2+}$-like & DAG & ATP & Substrate & C $P K C \delta, \varepsilon, \eta, \theta$ \\
\hline$K C$ & & $P$ & ATP & Substrate & C $P K C \zeta, 1 / \lambda$ \\
\hline
\end{tabular}

B

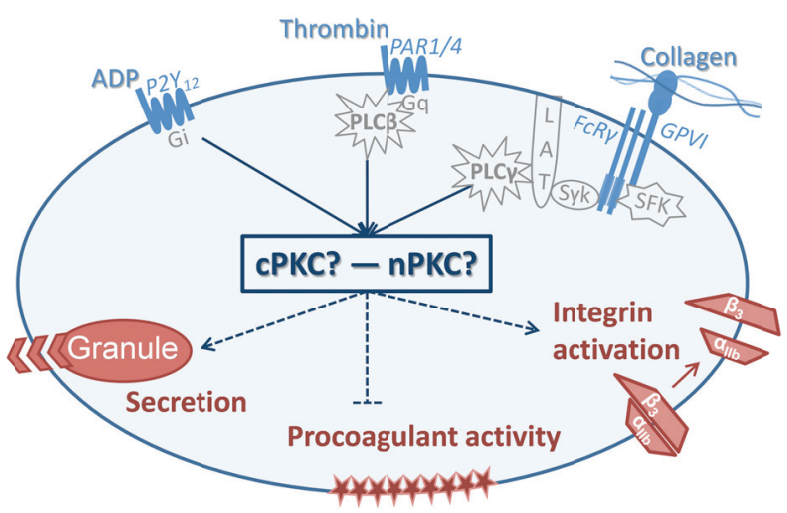

Figure 3. Domain structure and functions of protein kinase $C$ in platelet activation. $A$, Human and mouse platelets express PKC isoforms that are classified into three subgroups: the conventional (cPKC: $\mathrm{PKC} \alpha, \beta, \gamma)$, novel (nPKC: PKC $\delta, \varepsilon, \eta, \theta$ ), and atypical (aPKC: $\mathrm{PKC} \zeta, 1 / \lambda)$ PKC isoforms. All PKC isoforms contain a regulatory and catalytic region separated by a hinge sequence. Substrate and ATP-binding sites are located in the catalytic region near the C-terminal of the protein. The regulatory region differs among the three subgroups, and determines their response to agonists. CPKC isoforms have a diacylglycerol (DAG) and a $\mathrm{Ca}^{2+}$ binding site. nPKC isoforms lack the $\mathrm{Ca}^{2+}$ binding site, while the aPKC isoforms have a completely deviant regulatory region. $B$, In platelets stimulated with ADP, thrombin or collagen, unspecified cPKC or nPKC isoforms stimulate granule secretion and integrin $\alpha$ llb $\beta_{3}$ activation, whereas other(?) PKC isoforms suppress procoagulant activity by inhibiting PS exposure. See further, Figure 2.

Given the availability of mice deficient in $\mathrm{PKC} \alpha, \beta, \delta$ or $\theta$, and the presence of several PKC inhibitors with isoform selectivity, we found it important to combine pharmacological and genetic approaches to study the individual contribution of these major PKC isoforms in platelet activation. For a complete insight, in addition to determining integrin activation, secretion and thrombus formation, also their role in $\mathrm{Ca}^{2+}$ signaling and procoagulant activity should be addressed.

\section{Calcium flux-regulating proteins in platelet function}

The majority of platelet agonists signal to elevation of cytosolic $\mathrm{Ca}^{2+}$ concentration $(58,59)$, which is an indispensable event for secretion and integrin $\alpha$ llb $\beta_{3}$ activation, and, when this elevation is high and prolonged, for PS exposure and platelet procoagulant activity (Figure 4).

Increased cytosolic $\mathrm{Ca}^{2+}$ is one of the main triggers to provoke the active conformation of $\alpha \mathrm{llb} \beta_{3}$ in activated platelets. Currently, there are two models for the transition of this integrin from the resting to the high-affinity binding state, the latter of which is needed for 
fibrinogen binding. One activation model involves unclasping of the $\alpha$ llb and $\beta_{3}$ chains (10), the other model the tilting of $\beta_{3}$ ( 9 ) by intracellular binding of talin to the its transmembrane domain $(8,60)$. Integrin $\alpha \mathrm{llb} \beta_{3}$ receptors bound to fibrinogen molecules can form micro-clusters, allowing the formation of a cytosolic signaling complex that is capable to integrin outside-in signaling. In the signalosome, Src-family kinase members, including Src itself which is constitutively bound to the $\beta_{3}$ cytoplasmic tail, become activated to phosphorylate the protein kinase Syk, leading to phospholipase $C_{\gamma 2}$ activation. This effector enzyme causes reinforcement of the increase in $\mathrm{Ca}^{2+}$, which may lead to PS exposure.

Strikingly, the process of PS exposure, which occurs at distinct sites in a thrombus, is accompanied by closure of activated $\alpha$ llb $\beta_{3}$ integrins (Figure 4 ). How the latter process is regulated and whether $\mathrm{Ca}^{2+}$ is involved has remained unclear. An indirect indication for a role for $\mathrm{Ca}^{2+}$ in integrin closure is the finding that the $\mathrm{Ca}^{2+}$-dependent thiol protease, calpain, cleaves the cytoplasmic tail of the $\beta_{3}$ chain, an event that is not compatible with the activated conformational state of $\alpha \mathrm{llb} \beta_{3}(61)$. There may also be a role of mitochondrial membrane depolarization in $\mathrm{Ca}^{2+}$-dependent integrin closure, since also PS exposure is suggested to be under mitochondrial control (62). Evidence in favour of this idea also comes from the observation that mouse platelets lacking cyclophilin $D$, which is a component of the mitochondrial permeability transition pore (mPTP), are deficient in PS exposure in response to receptor agonists $(63,64)$. On the other hand, mouse platelets lacking other mitochondrial-associated proteins, i.e. the apoptosis-regulating factors Bax and Bad, have a normal rate of PS exposure, suggesting that the mitochondrial apoptosis pathway is regulated in a distinct way than agonist-induced PS exposure.

The platelet-activating conditions required for PS exposure and platelet procoagulant activity have extensively been investigated (65). Stimulation of the collagen receptor GPVI by itself results in a moderate procoagulant response $(66,67)$, while the combined stimulation of collagen and thrombin receptors results in high PS exposure, likely because these agonists use different signaling pathways (58). In mouse and human platelets, the conventional route for cytosolic $\mathrm{Ca}^{2+}$ elevation is by activation of phospholipase $\mathrm{C} \beta$ (evoked by $\mathrm{G}$ protein coupled receptors for ADP and thrombin) and of phospholipase $C_{\gamma 2}$ (evoked by GPVI). Both isoforms of phospholipase $C$, as described above, catalyze the hydrolysis of phosphatidylinositol 4,5-biphosphate into diacylglycerol and IP3. The latter interacts with $\mathrm{IP}_{3}$ receptor channels in the endoplasmic reticulum, which release $\mathrm{Ca}^{2+}$ from the intracellular stores. Mobilization of $\mathrm{Ca}^{2+}$ from these stores is accompanied by $\mathrm{Ca}^{2+}$ entry from the extracellular medium via a process, called store-operated $\mathrm{Ca}^{2+}$ entry (SOCE) (Figure 4). It has been recognized that SOCE is essential to reach sufficiently high $\mathrm{Ca}^{2+}$ levels for PS exposure $(58,68,69)$. The entry of $\mathrm{Ca}^{2+}$ can also be facilitated by another process, called receptor-operated $\mathrm{Ca}^{2+}$ entry (ROCE) (70). 


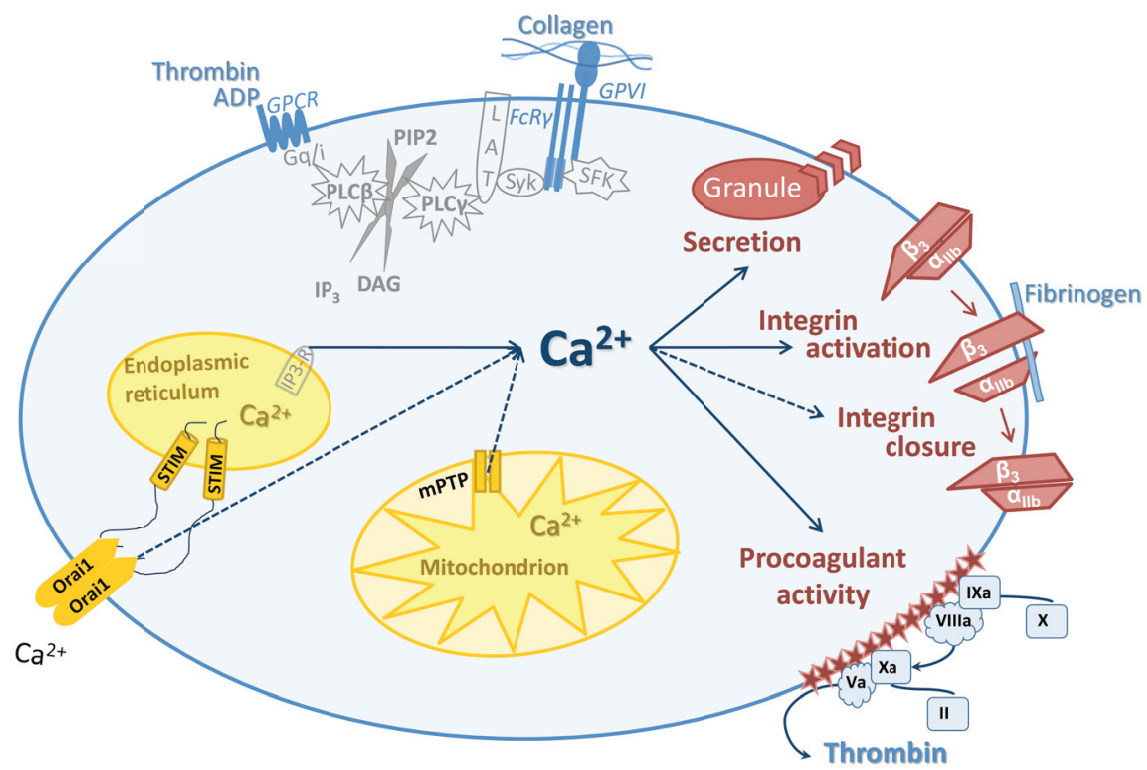

Figure 4. Central role of $\mathrm{Ca}^{2+}$ in platelet activation processes. Upon ADP, thrombin or collagen receptor activation, $\mathrm{PLC} \beta$ and $\gamma$ isoforms hydrolyze phosphatidylinositol 4,5 -biphosphate (PIP2) into inositol 1,4,5-trisphosphase (IP3) and diacylglycerol (DAG). The interaction of IP3 with its receptor (IP3R) elicits $\mathrm{Ca}^{2+}$ mobilization from intracellular stores in the endoplasmic reticulum. Subsequently, the transmembrane $\mathrm{Ca}^{2+}$-sensing protein STIM interacts with the plasma membrane $\mathrm{Ca}^{2+}$ channel, Orai1, to induce store-operated $\mathrm{Ca}^{2+}$ entry (SOCE), contributing to the $\mathrm{Ca}^{2+}$ rise. Mitochondria function as $\mathrm{Ca}^{2+}$ buffering systems, with the capacity for release, e.g. upon formation of the mitochondrial permeability transition pore (mPTP). Elevated $\mathrm{Ca}^{2+}$ stimulates integrin activation and secretion, while sustained high $\mathrm{Ca}^{2+}$ is required for PS exposure. The latter provides a procoagulant surface for activation of factor $\mathrm{X}$ (complex of factors VIIIa, IXa and X), and for activation of prothrombin (complex of factors II, Va and Xa). Procoagulant platelets have lost the capacity to bind fibrinogen through activated $\alpha$ llb $\beta_{3}$ integrins. How the integrins $\alpha$ llb $\beta_{3}$ are closed remains to be investigated. See further, Figure 2.

First studies to find the $\mathrm{Ca}^{2+}$ channel responsible for SOCE concentrated on members of the family of transient receptor potential channels (Trpc). In platelets, however, it soon appeared that Trpc6 acts as a diacylglycerol-activated non-SOCE channel (71). In work from other authors, the isoform Trpc1 was believed to be involved in SOCE (72), but this was negated later as described in this thesis. Major progress in this field was made with the discovery of the Orai class of plasma membrane $\mathrm{Ca}^{2+}$ channels. The isoform Orai1 (also called $\mathrm{CRACM}_{1}$ ) oligomerizes and opens following depletion of the $\mathrm{Ca}^{2+}$ stores, by interacting with the $\mathrm{STIM}_{1}$, which is a $\mathrm{Ca}^{2+}$-sensing transmembrane protein located in the endoplasmic reticulum $(73,74)$. Both Orai1 and STIM1 are expressed in human and mouse platelets, and both regulate platelet functions (Figure 4). Studies in other cell types suggest that the homologous protein STIM2 may have a similar regulatory role in $\mathrm{Ca}^{2+}$ entry (75). The precise role of $\mathrm{Ca}^{2+}$ entry via Orai1 and the STIM isoforms in $\mathrm{Ca}^{2+}$-dependent thrombus formation and platelet PS exposure and procoagulant activity remains to be elucidated. 


\section{Outline of this thesis}

This thesis aims to investigate different molecular signaling proteins and mechanisms, by which platelets contribute to thrombus formation and the hemostatic response. Particular attention is paid to the different signaling mechanisms involved in platelet aggregation and platelet procoagulant activity. The general introductory chapter 1 gives a brief overview of relevant signaling pathways underlying both responses, with emphasis on the crucial roles of the platelet signaling proteins, $\mathrm{Pl}_{3} \mathrm{~K}$ and $\mathrm{PKC}$, and the secondary mediator $\mathrm{Ca}^{2+}$. Furthermore, this chapter addresses the property of collagen as an important platelet-activating component, exposed in the extracellular matrix. Chapter 2 concentrates on the first stage of thrombus formation. Studied is how platelets at high (arterial) and low (venous) shear rates interact via their collagen receptors, GPVI and integrin $\alpha_{2} \beta_{1}$, using specifically designed triple-helical collagen-related peptides in the presence or absence of VWF. This work also assesses the suitability of collagen-related peptides as substitutes for native collagen surfaces to cause induction of thrombus formation under flow. The contribution of the various $\mathrm{Pl}_{3} \mathrm{~K}$ isoforms to collagen- and GPVI-induced thrombus formation is studied in chapter 3. Particular attention is paid to possible differences in functions between the class $\mathrm{IA}$ isoforms, $\mathrm{Pl}_{3} \mathrm{~K} \alpha$ and $\beta$, and the class IB isoform, $\mathrm{Pl}_{3} \mathrm{~K} \gamma$. It is also determined how $\mathrm{Pl}_{3} \mathrm{~K}$ isoforms contribute to activation of the small GTPase, Rap1b, and subsequent integrin $\alpha$ llb $\beta_{3}$ activation. The next two chapters focus on the PKC family of serine/threonine kinases with respect to platelet activation and thrombus formation. In chapter $\mathbf{4}_{\text {, a new knockout }}$ mouse is described to identify the function of the conventional isoform, $\mathrm{PKC} \alpha$, in platelet morphology, activation and thrombus formation. Chapter 5 combines genetic and pharmaceutical approaches to compare the functions of the four major PKC isoforms in platelet responses and thrombus formation. Particular attention is paid to possible functional differences between the conventional, diacylglycerol-dependent PKC isoforms $(P K C \alpha, \beta)$ and the novel, diacylglycerol-independent PKC isoforms (PKC $\delta, \theta)$. Given the key role of $\mathrm{Ca}^{2+}$ as a second messenger in platelet activation, chapter 6 presents a study regarding the importance of $\mathrm{Ca}^{2+}$ mobilization and subsequent $\mathrm{Ca}^{2+}$ entry in thrombus formation. In particular, this chapter considers the contribution of STIM1, the key regulatory protein in SOCE, and, of Orai1, a well-recognized SOCE channel, to the thrombotic process under conditions where coagulation is either blocked or promoted. Whereas a moderate elevation in cytosolic $\mathrm{Ca}^{2+}$ promotes integrin $\alpha$ llb $\beta_{3}$ activation, a potent $\mathrm{Ca}^{2+}$ rise causes PS exposure along with integrin closure. The regulation of $\alpha \mathrm{llb} \beta 3$ closure in PS-exposing platelets is the central theme of chapter 7. The experiments concentrate on the role of the $\mathrm{Ca}^{2+}$-dependent protease calpain and of relevant protein kinases. In addition, the role of mitochondria via MPTP formation is considered. The general discussion of chapter $\mathbf{8}$ describes the most important findings of this thesis in relation to the current literature. 


\section{References}

1 Hartwig JH. Platelet structure in Platelets, edited by A. D. Michelson. Academic Press, Elsevier Science, San Diego, USA, 2002, pp. 37-52.

2 Ruggeri ZM \& Mendolicchio GL. Adhesion mechanisms in platelet function. Circ Res 100, 167385 (2007).

3 Nieswandt B \& Watson SP. Platelet-collagen interaction: is GPVI the central receptor? Blood 102, 449-61 (2003).

4 Savage B, Saldivar E, \& Ruggeri ZM. Initiation of platelet adhesion by arrest onto fibrinogen or translocation on von Willebrand factor. Cell 84, 289-97 (1996).

5 Auger JM, Kuijpers MJ, Senis YA, Watson SP, \& Heemskerk JW. Adhesion of human and mouse platelets to collagen under shear: a unifying model. FASEB J 19, 825-7 (2005).

6 Siljander PR, Munnix IC, Smethurst PA, Deckmyn H, Lindhout T, Ouwehand WH, Farndale RW, \& Heemskerk JW. Platelet receptor interplay regulates collagen-induced thrombus formation in flowing human blood. Blood 103, 1333-41 (2004).

$7 \quad$ Kulkarni S, Dopheide SM, Yap CL, Ravanat C, Freund M, Mangin P, Heel KA, Street A, Harper IS, Lanza F, \& Jackson SP. A revised model of platelet aggregation. J Clin Invest 105, 783-91 (2000).

$8 \quad \mathrm{Ma} Y \mathrm{Y}$, Qin J, \& Plow EF. Platelet integrin $\alpha$ llb $\beta_{3}$ : activation mechanisms. J Thromb Haemost 5, 1345-52 (2007).

9 Kim C, Ye F, Hu X, \& Ginsberg MH. Talin activates integrins by altering the topology of the $\beta$ transmembrane domain. J Cell Biol 197, 605-11 (2012).

10 Cosemans JM, Iserbyt BF, Deckmyn H, \& Heemskerk JW. Multiple ways to switch platelet integrins on and off. J Thromb Haemost 6, 1253-61 (2008).

11 Li Z, Zhang G, Le Breton GC, Gao X, Malik AB, \& Du X. Two waves of platelet secretion induced by thromboxane $A_{2}$ receptor and a critical role for phosphoinositide 3-kinases. J Biol Chem 278, 30725-31 (2003).

12 Gachet C. ADP receptors of platelets and their inhibition. Thromb Haemost 86, 222-32 (2001).

13 Oury C, Kuijpers MJ, Toth-Zsamboki E, Bonnefoy A, Danloy S, Vreys I, Feijge MA, De Vos R, Vermylen J, Heemskerk JW, \& Hoylaerts MF. Overexpression of the platelet $\mathrm{P}_{2} \mathrm{X}_{1}$ ion channel in transgenic mice generates a novel prothrombotic phenotype. Blood 101, 3969-76 (2003).

14 Munnix IC, Kuijpers MJ, Auger J, Thomassen CM, Panizzi P, van Zandvoort MA, Rosing J, Bock PE, Watson SP, \& Heemskerk JW. Segregation of platelet aggregatory and procoagulant microdomains in thrombus formation: regulation by transient integrin activation. Arterioscler Thromb Vasc Biol 27, 2484-90 (2007).

15 Hemker HC. Thrombin generation, an essential step in haemostasis and thrombosis in Haemostasis and thrombosis edited by A.L. Bloom, C.D. Forbes, D.P. Thomas, and E.G. Tuddenham. Churchill Linvingstone, Edinburgh, 1994, pp. 477-90.

16 Cosemans JM, Kuijpers MJ, Lecut C, Loubele ST, Heeneman S, Jandrot-Perrus M, \& Heemskerk JW. Contribution of platelet glycoprotein VI to the thrombogenic effect of collagens in fibrous atherosclerotic lesions. Atherosclerosis 181, 19-27 (2005).

17 Inoue O, Suzuki-Inoue K, Dean WL, Frampton J, \& Watson SP. Integrin a2b1 mediates outside-in regulation of platelet spreading on collagen through activation of Src kinases and PLC $\gamma 2$. J Cell Biol 160, 769-80 (2003).

18 Nieswandt B, Brakebusch C, Bergmeier W, Schulte V, Bouvard D, Mokhtari-Nejad R, Lindhout T, Heemskerk JW, Zirngibl H, \& Fassler R. Glycoprotein VI but not $\alpha_{2} \beta_{1}$ integrin is essential for platelet interaction with collagen. EMBO J 20, 2120-30 (2001).

19 Farndale RW, Sixma JJ, Barnes MJ, \& de Groot PG. The role of collagen in thrombosis and hemostasis. J Thromb Haemost 2, 561-73 (2004).

20 Smethurst PA, Onley DJ, Jarvis GE, O'Connor MN, Knight CG, Herr AB, Ouwehand WH, \& Farndale RW. Structural basis for the platelet-collagen interaction: The smallest motif within collagen that recognizes and activates platelet glycoprotein VI contains two glycine-prolinehydroxyproline triplets. J Biol Chem 282, 1296-304 (2007).

21 Knight CG, Morton LF, Peachey AR, Tuckwell DS, Farndale RW, \& Barnes MJ. The collagenbinding $A$-domains of integrins $\alpha_{1} \beta_{1}$ and $\alpha_{2} \beta_{1}$ recognize the same specific amino acid sequence, GFOGER, in native (triple-helical) collagens. J Biol Chem 275, 35-40 (2000). 
Siljander PR, Hamaia S, Peachey AR, Slatter DA, Smethurst PA, Ouwehand WH, Knight CG, \& Farndale RW. Integrin activation state determines selectivity for novel recognition sites in fibrillar collagens. J Biol Chem 279, 47763-72 (2004).

23 Xu Y, Gurusiddappa S, Rich RL, Owens RT, Keene DR, Mayne R, Hook A, \& Hook M. Multiple binding sites in collagen type I for the integrins $\alpha_{1} \beta_{1}$ and $\alpha_{2} \beta_{1}$. J Biol Chem 275, 38981-9 (2000).

24 Jackson SF \& Schoenwaelder SM. Type I phosphoinositide 3-kinases: potential antithrombotic targets? Cell Mol Life Sci 63, 1085-90 (2006).

25 Gibbins J, Asselin J, Farndale R, Barnes M, Law CL, \& Watson SP. Tyrosine phosphorylation of the Fc receptor $Y$-chain in collagen-stimulated platelets. J Biol Chem 271, 18095-9 (1996).

26 Suzuki-Inoue K, Tulasne D, Shen Y, Bori-Sanz T, Inoue O, Jung SM, Moroi M, Andrews RK, Berndt MC, \& Watson SP. Association of Fyn and Lyn with the proline-rich domain of glycoprotein VI regulates intracellular signaling. J Biol Chem 277, 21561-6 (2002).

27 Asselin J, Gibbins JM, Achison M, Lee YH, Morton LF, Farndale RW, Barnes MJ, \& Watson SP. A collagen-like peptide stimulates tyrosine phosphorylation of syk and phospholipase $\mathrm{C}_{22}$ in platelets independent of the integrin $\alpha_{2} \beta_{1}$. Blood 89, 1235-42 (1997).

28 Gibbins JM, Briddon S, Shutes A, van Vugt MJ, van de Winkel JG, Saito T, \& Watson SP. The p85 subunit of phosphatidylinositol 3 -kinase associates with the Fc receptor $\gamma$-chain and linker for activitor of T cells in platelets stimulated by collagen and convulxin. J Biol Chem 273, 34437-43 (1998).

29 Watson SP, Auger JM, McCarty OJ, \& Pearce AC. GPVI and integrin $\alpha$ llb $\beta_{3}$ signaling in platelets. J Thromb Haemost 3, 1752-62 (2005).

30 Pasquet JM, Bobe R, Gross B, Gratacap MP, Tomlinson MG, Payrastre B, \& Watson SP. A collagen-related peptide regulates phospholipase $\mathrm{Cg} 2$ via phosphatidylinositol 3-kinase in human platelets. Biochem J 342 ( Pt 1), 171-7 (1999).

31 Cosemans JM, Munnix IC, Wetzker R, Heller R, Jackson SP, \& Heemskerk JW. Continuous signaling via $\mathrm{Pl}_{3} \mathrm{~K}$ isoforms $\beta$ and $\gamma$ is required for platelet $\mathrm{ADP}$ receptor function in dynamic thrombus stabilization. Blood 108, 3045-52 (2006).

32 Hirsch E, Bosco O, Tropel P, Laffargue M, Calvez R, Altruda F, Wymann M, \& Montrucchio G. Resistance to thromboembolism in $\mathrm{Pl}_{3} \mathrm{~K} Y$-deficient mice. FASEB J 15, 2019-21 (2001).

33 Jackson SP, Schoenwaelder SM, Goncalves I, Nesbitt WS, Yap CL, Wright $C E$, Kenche $V$, Anderson KE, Dopheide SM, Yuan $Y$, Sturgeon SA, Prabaharan $H$, Thompson PE, Smith GD, Shepherd PR, Daniele N, Kulkarni S, Abbott B, Saylik D, Jones C, Lu L, Giuliano S, Hughan SC, Angus JA, Robertson $A D, \&$ Salem HH. Pl 3-kinase $p 110 \beta$ : a new target for antithrombotic therapy. Nat Med 11, 507-14 (2005).

34 Schoenwaelder SM, Ono A, Sturgeon S, Chan SM, Mangin P, Maxwell MJ, Turnbull S, Mulchan dani M, Anderson K, Kauffenstein G, Rewcastle GW, Kendall J, Gachet C, Salem HH, \& Jackson SP. Identification of a unique co-operative phosphoinositide 3-kinase signaling mechanism regulating integrin $\alpha \mathrm{llb} \beta_{3}$ adhesive function in platelets. J Biol Chem 282, 28648-58 (2007).

35 Lova P, Paganini S, Hirsch E, Barberis L, Wymann M, Sinigaglia F, Balduini C, \& Torti M. A selective role for phosphatidylinositol 3,4,5-trisphosphate in the Gi-dependent activation of platelet Rap1B. J Biol Chem 278, 131-8 (2003).

36 Woulfe $D$, Jiang $H$, Mortensen R, Yang J, \& Brass LF. Activation of Rap1B by Gi family members in platelets. J Biol Chem 277, 23382-90 (2002).

37 Parker PJ \& Murray-Rust J. PKC at a glance. J Cell Sci 117, 131-2 (2004).

38 Baldassare JJ, Henderson PA, Burns D, Loomis C, \& Fisher GJ. Translocation of protein kinase C isozymes in thrombin-stimulated human platelets. Correlation with 1,2-diacylglycerol levels. J Biol Chem 267, 15585-90 (1992).

39 Crabos $M$, Imber R, Woodtli T, Fabbro D, \& Erne P. Different translocation of three distinct PKC isoforms with tumor-promoting phorbol ester in human platelets. Biochem Biophys Res Commun $178,878-83$ (1991).

40 Grabarek J, Raychowdhury M, Ravid K, Kent KC, Newman PJ, \& Ware JA. Identification and functional characterization of protein kinase $C$ isozymes in platelets and HEL cells. J Biol Chem 267, 10011-7 (1992).

41 Khan WA, Blobe G, Halpern A, Taylor W, Wetsel WC, Burns D, Loomis C, \& Hannun YA. Selective regulation of protein kinase $C$ isoenzymes by oleic acid in human platelets. J Biol Chem 268, 5063-8 (1993). 
42 Wang F, Naik UP, Ehrlich YH, Freyberg Z, Osada S, Ohno S, Kuroki T, Suzuki K, \& Kornecki E. A new protein kinase $\mathrm{C}, \mathrm{nPKC} \eta$ and $\mathrm{nPKC} \theta$ are expressed in human platelets: involvement of $\mathrm{nPKC} \eta$ and nPKC $\theta$ in signal transduction stimulated by PAF. Biochem Biophys Res Commun 191, 240-6 (1993).

43 Hers I, Donath J, van Willigen G, \& Akkerman JW. Differential involvement of tyrosine and serine/threonine kinases in platelet integrin $\alpha$ llb $\beta_{3}$ exposure. Arterioscler Thromb Vasc Biol 18, 40414 (1998).

Quinton TM, Kim S, Dangelmaier C, Dorsam RT, Jin J, Daniel JL, \& Kunapuli SP. Protein kinase Cand calcium-regulated pathways independently synergize with Gi pathways in agonist-induced fibrinogen receptor activation. Biochem J 368, 535-43 (2002).

45 Toullec D, Pianetti P, Coste H, Bellevergue $\mathrm{P}$, Grand-Perret $\mathrm{T}$, Ajakane M, Baudet V, Boissin P, Boursier E, Loriolle F, Duhamel L, Charon D, \& Kirikovsky J. The bisindolylmaleimide GF109203X is a potent and selective inhibitor of protein kinase C. J Biol Chem 266, 15771-81 (1991).

46 Walker TR \& Watson SP. Synergy between $\mathrm{Ca}^{2+}$ and protein kinase $\mathrm{C}$ is the major factor in determining the level of secretion from human platelets. Biochem J 289, 277-82 (1993).

47 Cavallini L \& Alexandre A. $\mathrm{Ca}^{2+}$ efflux from platelets. Control by protein kinase $\mathrm{C}$ and the filling state of the intracellular $\mathrm{Ca}^{2+}$ stores. Eur J Biochem 222, $693-702$ (1994).

48 Rink TJ \& Sage SO. Calcium signaling in human platelets. Annu Rev Physiol 52, 431-49 (1990).

49 Strehl A, Munnix IC, Kuijpers MJ, van der Meijden PE, Cosemans JM, Feijge MA, Nieswandt B, \& Heemskerk JW. Dual role of platelet protein kinase $C$ in thrombus formation: stimulation of proaggregatory and suppression of procoagulant activity in platelets. J Biol Chem 282, 7046-55 (2007).

50 Yoshioka A, Shirakawa R, Nishioka H, Tabuchi A, Higashi T, Ozaki H, Yamamoto A, Kita T, \& Horiuchi $\mathrm{H}$. Identification of protein kinase $\mathrm{C} \alpha$ as an essential, but not sufficient, cytosolic factor for $\mathrm{Ca}^{2+}$-induced a- and d-core granule secretion in platelets. J Biol Chem 276, 39379-85 (2001).

51 Tabuchi A, Yoshioka A, Higashi T, Shirakawa R, Nishioka H, Kita T, \& Horiuchi H. Direct demonstration of involvement of protein kinase $\mathrm{C} \alpha$ in the $\mathrm{Ca}^{2+}$-induced platelet aggregation. J Biol Chem 278, 26374-9 (2003).

52 Pula G, Crosby D, Baker J, \& Poole AW. Functional interaction of protein kinase C $\alpha$ with the tyrosine kinases Syk and Src in human platelets. J Biol Chem 280, 7194-205 (2005).

53 Buensuceso CS, Obergfell A, Soriani A, Eto K, Kiosses WB, Arias-Salgado EG, Kawakami T, \& Shattil SJ. Regulation of outside-in signaling in platelets by integrin-associated protein kinase $C \beta$. J Biol Chem 280, 644-53 (2005).

54 Chari R, Getz T, Nagy B, Jr., Bhavaraju K, Mao Y, Bynagari YS, Murugappan S, Nakayama K, \& Kunapuli SP. Protein kinase $C \delta$ differentially regulates platelet functional responses. Arterioscler Thromb Vasc Biol 29, 699-705 (2009).

55 Hall KJ, Harper MT, Gilio K, Cosemans JM, Heemskerk JW, \& Poole AW. Genetic analysis of the role of protein kinase $C \theta$ in platelet function and thrombus formation. PLoS One 3, e3277 (2008).

56 Nagy B, Jr., Bhavaraju K, Getz T, Bynagari YS, Kim S, \& Kunapuli SP. Impaired activation of platelets lacking protein kinase $C \theta$ isoform. Blood 113, 2557-67 (2009).

57 Pula G, Schuh K, Nakayama K, Nakayama KI, Walter U, \& Poole AW. PKC $\delta$ regulates collageninduced platelet aggregation through inhibition of VASP-mediated filopodia formation. Blood 108, 4035-44 (2006).

58 Heemskerk JW, Bevers EM, \& Lindhout T. Platelet activation and blood coagulation. Thromb Haemost 88, 186-93 (2002).

59 Heemskerk JW, Feijge MA, Henneman L, Rosing J, \& Hemker HC. The $\mathrm{Ca}^{2+}$-mobilizing potency of a-thrombin and thrombin-receptor-activating peptide on human platelets - concentration and time effects of thrombin-induced $\mathrm{Ca}^{2+}$ signaling. Eur J Biochem 249, 547-55 (1997).

60 Partridge AW, Liu S, Kim S, Bowie JU, \& Ginsberg MH. Transmembrane domain helix packing stabilizes integrin $\alpha$ llb $\beta_{3}$ in the low affinity state. J Biol Chem 280, 7294-300 (2005).

61 Kulkarni S \& Jackson SP. Platelet factor XIII and calpain negatively regulate integrin $\alpha \mathrm{llb} \beta_{3}$ adhesive function and thrombus growth. J Biol Chem 279, 30697-706 (2004).

62 Arachiche A, Kerbiriou-Nabias D, Garcin I, Letellier T, \& Dachary-Prigent J. Rapid procoagulant phosphatidylserine exposure relies on high cytosolic calcium rather than on mitochondrial depolarization. Arterioscler Thromb Vasc Biol 29, 1883-9 (2009). 
63 Baines CP, Kaiser RA, Purcell NH, Blair NS, Osinska H, Hambleton MA, Brunskill EW, Sayen MR, Gottlieb RA, Dorn GW, Robbins J, \& Molkentin JD. Loss of cyclophilin D reveals a critical role for mitochondrial permeability transition in cell death. Nature 434, 658-62 (2005).

64 Jobe SM, Wilson KM, Leo L, Raimondi A, Molkentin JD, Lentz SR, \& Di Paola J. Critical role for the mitochondrial permeability transition pore and cyclophilin $D$ in platelet activation and thrombosis. Blood 111, 1257-65 (2008).

65 Munnix IC, Cosemans JM, Auger JM, \& Heemskerk JW. Platelet response heterogeneity in thrombus formation. Thromb Haemost 102, 1149-56 (2009).

66 Siljander P, Farndale RW, Feijge MA, Comfurius P, Kos S, Bevers EM, \& Heemskerk JW. Platelet adhesion enhances the glycoprotein VI-dependent procoagulant response: Involvement of p38 MAP kinase and calpain. Arterioscler Thromb Vasc Biol 21, 618-27 (2001).

67 Thiagarajan P \& Tait JF. Collagen-induced exposure of anionic phospholipid in platelets and platelet-derived microparticles. J Biol Chem 266, 24302-7 (1991).

68 Bevers EM, Comfurius P, van Rijn JL, Hemker HC, \& Zwaal RF. Generation of prothrombinconverting activity and the exposure of phosphatidylserine at the outer surface of platelets. Eur J Biochem 122, 429-36 (1982).

69 Dachary-Prigent J, Pasquet JM, Freyssinet JM, \& Nurden AT. Calcium involvement in aminophospholipid exposure and microparticle formation during platelet activation: a study using $\mathrm{Ca}^{2+}$. ATPase inhibitors. Biochemistry 34, 11625-34 (1995).

70 Sage SO. The wellcome prize lecture. Calcium entry mechanisms in human platelets. Exp Physiol 82, 807-23 (1997).

71 Hassock SR, Zhu MX, Trost C, Flockerzi V, \& Authi KS. Expression and role of TRPC proteins in human platelets: evidence that TRPC6 forms the store-independent calcium entry channel. Blood 100, 2801-11 (2002).

72 Huang GN, Zeng W, Kim JY, Yuan JP, Han L, Muallem S, \& Worley PF. STIM1 carboxyl-terminus activates native SOC, I(crac) and TRPC1 channels. Nat Cell Biol 8, 1003-10 (2006).

73 Soboloff J, Spassova MA, Tang XD, Hewavitharana T, Xu W, \& Gill DL. Orai1 and STIM reconstitute store-operated calcium channel function. J Biol Chem 281, 20661-5 (2006).

74 Zhang SL, Kozak JA, Jiang W, Yeromin AV, Chen J, Yu Y, Penna A, Shen W, Chi V, \& Cahalan MD. Store-dependent and -independent modes regulating $\mathrm{Ca}^{2+}$ release-activated $\mathrm{Ca}^{2+}$ channel activity of human Orai1 and Orai3. J Biol Chem 283, 17662-71 (2008).

75 Parvez S, Beck A, Peinelt C, Soboloff J, Lis A, Monteilh-Zoller M, Gill DL, Fleig A, \& Penner R. $\mathrm{STIM} 2$ protein mediates distinct store-dependent and store-independent modes of CRAC channel activation. FASEB J 22, 752-61 (2008). 


\section{Chapter 2}

Collagen-mimetic peptides mediate flow-dependent
thrombus formation by high-or low-affinity binding of
integrin $\alpha 2 \beta i$ and glycoprotein VI

Munnix IC, Gilio K, Siljander PR, Raynal N, Feijge MA, Hackeng TM, Deckmyn H, Smethurst PA, Farndale RW, and Heemskerk JW

J Thromb Haemost. 2008;6:2132-42

Reprinted with permission 


\begin{abstract}
Collagen acts as a potent surface for platelet adhesion and thrombus formation under conditions of blood flow. Studies using collagen-derived triple-helical peptides have identified the GXX'GER motif as an adhesive ligand for platelet integrin $\alpha_{2} \beta_{1}$, and $(G P O)_{n}$ as a binding sequence for the signaling collagen receptor, glycoprotein VI (GPVI). The potency was investigated of triple-helical peptides, consisting of GXX'GER sequences within (GPO) ${ }_{n}$ or $(G P P)_{n}$ motifs, to support flow-dependent thrombus formation. At high shear rate, immobilised peptides containing both the high affinity $\alpha_{2} \beta_{1}$-binding motif GFOGER and the (GPO) motif supported platelet aggregation and procoagulant activity, even in the absence of von Willebrand factor (VWF). With peptides containing only one of these motifs, co-immobilised vWF was needed for thrombus formation. The (GPO) $n$ but not the (GPP) $)_{n}$ sequence induced GPVI-dependent platelet aggregation and procoagulant activity. Peptides with intermediate affinity (GLSGER, GMOGER) or low-affinity (GASGER, GAOGER) $\alpha_{2} \beta_{1}$-binding motifs formed procoagulant thrombi only if both $(\mathrm{GPO})_{\mathrm{n}}$ and vWF were present. At low shear rate, immobilised peptides with high- or low-affinity $\alpha_{2} \beta_{1}$-binding motifs mediated formation of thrombi with procoagulant platelets only in combination with (GPO) $)_{n}$. In conclusion, triplehelical peptides with specific receptor-binding motifs mimic the properties of native collagen I in thrombus formation by binding to platelet collagen receptors. At high shear rate, either GPIb or high-affinity (but not low-affinity) GXX'GER mediates GPVI-dependent formation of procoagulant thrombi. By extension, high-affinity binding for $\alpha_{2} \beta_{1}$ can control the overall platelet-adhesive activity of native collagens.
\end{abstract}

\title{
Introduction
}

Platelet interaction with subendothelial collagen is a key initial step in thrombus formation following vessel wall damage. At an intermediate or high shear rates, the von Willebrand factor (VWF) receptor, glycoprotein (GP) Ib-IX-V, tethers platelets to VWF that is bound to collagen fibres $(1,2)$. Subsequent stable platelet adhesion and activation on collagen involves two collagen receptors, the immunoglobulin-like receptor GPVI and integrin $\alpha_{2} \beta_{1}$. We and others have shown that thrombus formation on collagen/vWF depends on concerted interplay between these receptors, where GPIb in synergy with $\alpha_{2} \beta_{1}$ or GPVI mediates adhesion, while $\alpha_{2} \beta_{1}$ reinforces signaling through GPVI to cause platelet activation (3-6). Release of the autocrine mediators, ADP and thromboxane $A 2$, plays a key supportive role in the recruitment and capture of passing platelets. Together with GPVI, these mediators induce affinity changes of integrins $\alpha_{\mathrm{llb}} \beta_{3}$ and $\alpha_{2} \beta_{1}$, which are required for stable interactions of platelets with fibrinogen and collagen, respectively, and hence, control thrombus stability under flow conditions (7-9). In addition, increases in cytosolic $\mathrm{Ca}^{2+}$ caused by signaling via GPVI triggers the platelet procoagulant response, by stimulating surface exposure of phosphatidylserine (PS). This reaction strongly potentiates local formation of thrombin and establishes coagulation $(5,10)$.

The precise role of integrin $\alpha_{2} \beta_{1}$ in collagen-induced thrombus formation however is still under debate. There is evidence from adhesion experiments that activated $\alpha_{2} \beta_{1}$ can stimulate platelets by triggering signaling events similar to those of GPVI (11). On the other 
hand, it is published that binding to $\alpha_{2} \beta_{1}$ passively enforces platelet activation via GPVI, and perhaps GPIb $(3,7,12)$. A complicating factor in establishing the precise function of $\alpha_{2} \beta_{1}$ in platelet-collagen interaction is the recent discovery that type I and III collagens contain various integrin-binding motifs with very different affinity for the resting and activated conformations of platelet $\alpha_{2} \beta_{1}$ (13). Thus, heterogeneity in adhesive sites may explain why unstimulated platelets can adhere to collagen in the apparent absence of activated $\alpha_{2} \beta_{1}$.

Identification of the minimal binding sequences for $\alpha_{2} \beta_{1}$ and GPVI within the collagen triple helix is a challenging task in hemostasis and thrombosis research. For more than a decade, it has been recognised that GPVI binds to and is activated by (GPO) n sequences (where $O$ is hydroxyproline) $(14,15)$. Indeed, a cross-linked form of triple-helical (GPO) known as collagen-related peptide (CRP), is widely used as a specific GPVI-activating platelet agonist. Recent work has shown that two or more GPO triplets are required to promote platelet aggregation through GPVI (16). For integrin $\alpha_{2} \beta_{1}$, several recognition motifs have been identified within the platelet-activating collagens, types I, III and IV. These include GXX'GER motifs, of which GFOGER is found in collagens I and IV, while GMOGER, GLOGER and GASGER are present in the collagen type I $\alpha_{1}$ chain; and further, GMOGER, GLSGER and GAOGER are expressed in collagen type III $(13,17,18)$. These GXX'GER motifs appear to interact with the metal-ion dependent adhesion site (MIDAS) of the integrin $\alpha 2$ chain (19). Earlier studies have shown that peptides containing GXX'GER motifs bind with different affinities to integrin $\alpha_{2} \beta_{1}$, and can be used to model collagen- $\alpha_{2} \beta_{1}$ interactions $(13,16)$.

Here, it was investigated how triple-helical GXX'GER peptides with high, intermediate or low affinity to $\alpha_{2} \beta_{1}$ support flow-dependent platelet adhesion and thrombus formation.

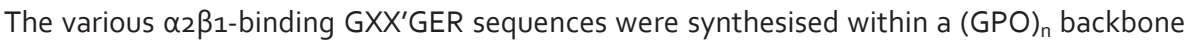
to provide synergy with GPVI activation, or within a (GPP) $n_{n}$ backbone as a GPVIindependent control. These peptides were immobilised to glass in the presence or absence of VWF to establish potential synergism with platelet GPIb interactions under high shear.

\section{Experimental procedures}

\section{Materials}

Fibrillar type I collagen (Horm) from equine tendon was purchased from Nycomed (Munich, Germany). Bovine type III collagen was from Sigma (St. Louis, MO, USA); the preparation was dialyzed overnight against phosphate-buffered saline (PBS; in mmol/l: $\mathrm{KCl} 2.7, \mathrm{KH}_{2} \mathrm{PO}_{4}$ 1.5, $\mathrm{NaCl}$ 100, $\mathrm{Na}_{2} \mathrm{HPO}_{4} 7.3, \mathrm{pH} 8.0$ ), and allowed to polymerize by a 5-min incubation at $35^{\circ} \mathrm{C}$ before use. D-phenylalanyl-L-prolyl-L-arginine chloromethylketone (PPACK) and calcein were from Calbiochem (La Jolla, CA, USA). Human vWF isolated from plasma was from LFB (Les Ulis, France). Lyophilised VWF protein was used at a final concentration of $0.35 \mathrm{~g} \mathrm{~L}^{-1}$ in Hepes buffer, $\mathrm{pH} 7.45\left(136 \mathrm{mmol} \mathrm{L}^{-1} \mathrm{NaCl}, 10 \mathrm{mmol} \mathrm{L}{ }^{-1}\right.$ Hepes, $2.7 \mathrm{mmol} \mathrm{L}{ }^{-1} \mathrm{KCl}$ and $2 \mathrm{mmol} \mathrm{L}^{-1} \mathrm{MgCl}_{2}, 1 \mathrm{~g} \mathrm{~L}^{-1}$ bovine serum albumin, and $1 \mathrm{~g} \mathrm{~L}^{-1}$ glucose). Unfractionated and low-molecular weight heparin came from Sigma. The GPVI-specific single-chain variable fragment (scFv) antibody 10B12 was selected from the Cambridge Antibody Technology (Medlmmune Cambridge) phage display libraries, as described (20). Fab fragments of the blocking monoclonal antibodies (mAb) $6 \mathrm{~B}_{4}$ against $\mathrm{GPIb} \alpha$ and ${ }_{15} \mathrm{D}_{7}$ against integrin $\alpha_{2} \beta_{1}$ 
were generated as before $(9,21)$. FITC-labelled annexin $A_{5}$ was from Nexins Research (Hoeven, the Netherlands); FITC-labelled monoclonal antibody against human P-selectin $(\alpha-C D 62 \mathrm{mAb}$ ) from WAK Chemie (Steinbach, Germany). Polyclonal rabbit anti-human vWF antibody was from Dako (Glostrup, Denmark); FITC-labelled goat anti-rabbit IgG (GARFITC) from Southern Biotech (Birmingham, AL, USA). Sources of other chemicals were as described (5).

\section{Peptide synthesis}

Collagen-like peptides were synthesised as C-terminal amides on TentaGel R-RAM resin in a Applied Biosystems Pioneer automated synthesizer, using Fmoc [N-(9-fluorenyl) methoxycarbonyl] chemistry $(22,23)$. Peptide melting temperature and peptide cross-linking of purified peptides were assessed as described (16). Cross-linked CRP, i.e. triple-helical (GPO)10, was synthesised as before (22). The $\alpha_{2} \beta_{1}$-binding peptides had the general struc-

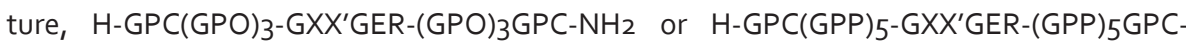
$\mathrm{NH} 2$, where amino acids $X$ and $X^{\prime}$ may differ between peptides. Triple-helical conformation was maintained by the GPO (GPVI-binding) or GPP (non-GPVI-binding) triplets, introduced at each end of the sequence. In this paper, the triple-helical peptides are indicated as GXX'GER-(GPO) ${ }_{n}$ and GXX'GER-(GPP) $n$. Table 1 gives the full amino acid composition.

\section{Preparation of adhesive surfaces}

Pre-cleaned glass coverslips were coated with one of the collagen-mimicking peptides. For static platelet adhesion, peptides were coated using a solution of $0.1-1000 \mathrm{~g} \mathrm{~mL}^{-1}$. For flow studies, coating was mostly with $5 \mu \mathrm{L}$ of a $1 \mathrm{mg} \mathrm{mL}^{-1}$ solution applied on a surface area of 15 $\mathrm{mm}^{2}$. In case of (co-)coating with $v W F$, mixtures were prepared containing $0.5 \mathrm{mg} \mathrm{mL}^{-1}$ of peptide and/or $v W F$, and $5 \mu \mathrm{L}$ of this mixture was applied to an area of $15 \mathrm{~mm}^{2}$. Coating was during $1 \mathrm{~h}$ in a humid chamber, after which the coverslips were rinsed twice with saline to remove the unbound peptide (vWF). Blocking was with Hepes buffer $\mathrm{pH} 7.45$ containing $1 \mathrm{~g}$ $\mathrm{L}^{-1}$ bovine serum albumin. Markers at the bottom side of the coverslips indicated the site of coating. In order to quantify the immobilisation of peptides, coverslips were coated with multiple spots of one peptide $\left(0.5 \mathrm{mg} \mathrm{mL}^{-1}\right)$, as described above. The adhered peptide was extracted from the glass by repetitive treatment with $6 \mathrm{~mol} \mathrm{~L}^{-1}$ guanidine in $100 \mathrm{mmol} \mathrm{L}^{-1}$ sodium acetate buffer. The collected material was reduced with $3 \%$ ß-mercaptoethanol and applied to reversed-phase HPLC. Peptides were eluted in a linear gradient of water/acetonitrile with $0.1 \%$ trifluoroacetic acid, and peaks were analysed by electrospray ionization quadrupole mass spectrometry (ESI-MS). Calibration with purified reduced peptide on HPLC was used to determine the amount of immobilized peptide on coverslip.

\section{Blood collection and flow experiments}

Blood was taken from healthy volunteers and collected into 0.1 vol saline containing 40 $\mu \mathrm{mol} \mathrm{L}{ }^{-1}$ PPACK, and supplemented hourly with $20 \mu \mathrm{mol} \mathrm{L}^{-1}$ PPACK. Blood was used within $3.5 \mathrm{~h}\left(37^{\circ} \mathrm{C}\right)$, in which time interval platelet count and platelet adhesion tendency remained unaltered (5). No formation of fibrin (clot) was detected in the blood, and D-dimer levels 
remained below the threshold value of $200 \mathrm{ng} \mathrm{mL}^{-1}$. Furthermore, extra addition of lowmolecular weight heparin (fragmin, 5 unit $\mathrm{mL}^{-1}$ ) did not influence the experimental outcome. Donors had not taken aspirin or related drugs for at least two weeks, and gave full informed consent.

Whole-blood flow experiments were performed, as described in detail elsewhere $(5,7)$. Briefly, blood was placed in a syringe and perfused over a peptide-coated coverslip through a transparent, $50 \mu \mathrm{m}$ deep and $3 \mathrm{~mm}$ wide parallel-plate flow chamber, using a pulse-free pump at a (wall) shear rate of $1000 \mathrm{~s}^{-1}$ for 4 min or $150 \mathrm{~s}^{-1}$ for $6 \mathrm{~min}$. Corresponding blood flow rates were $4.5 \mathrm{~mL} \mathrm{~h}^{-1}$ or $0.675 \mathrm{~mL} \mathrm{~h}^{-1}$. Where indicated, the blood was pre-incubated for 15 min with scFv mAb $10 B_{12}$ (blocking GPVI) or with Fab fragments of $6 \mathrm{~B}_{4}$ (blocking GPIb) or ${ }_{15} \mathrm{D}_{7}$ (blocking $\alpha_{2} \beta_{1}$ ) mAb. Coverslips were post-stained with FITC-annexin $A_{5}(0.5 \mu \mathrm{g}$ $\mathrm{mL}^{-1}$ ) or FITC- $\alpha-C D 62 \mathrm{mAb}\left(1.25 \mu \mathrm{g} \mathrm{mL}^{-1}\right)$ by a 4 -min perfusion at the same flow rate with Hepes buffer $\mathrm{pH} 7.45$, supplemented with 1 unit $\mathrm{mL}^{-1}$ heparin and $2 \mathrm{mmol} \mathrm{L}^{-1} \mathrm{CaCl}_{2}$. This staining did not change the size of platelet aggregates on the coverslip. A Visitech imaging system (Sunderland, UK), equipped with two intensified, charged-coupled device cameras, was used to collect phase-contrast and fluorescence images (24). Confocal fluorescence images were generated as described (10).

Image analysis

Size of recorded phase-contrast image was $120 \times 120 \mu \mathrm{m}$; size of fluorescence images was $150 \times 150 \mu \mathrm{m}$. Surface area coverage was analysed with Image-Pro software (Media Cybernetics) and Quanticell software (Visitech), respectively.

\section{Static platelet adhesion}

Adhesion of washed platelets $\left(1.25 \times 10^{8} \mathrm{~mL}^{-1}\right)$ to coated surfaces was determined colorimetrically (absorbance $405 \mathrm{~nm}$ ), as described (25).

\section{Statistical analysis}

Differences were tested on significance with the Mann-Whitney $U$ test using the statistical package for social sciences (SPSS 11.0, Chicago, IL, USA). Results are given as mean values \pm SEM, unless otherwise indicated.

\section{Results}

Efficacy of coating with triple-helical GXX'GER-(GPO) $)_{n}$ and $G X X^{\prime} G E R-(G P P)_{n}$ peptides

Triple-helical collagen-mimetic peptides were synthesised with high-affinity (GFOGER, GLOGER), intermediate affinity (GLSGER, GMOGER) or low affinity (GASGER, GAOGER) binding to $\alpha_{2} \beta_{1}$; these motifs were either within a GPVI-binding $(G P O)_{n}$ backbone or a nonGPVI-binding (GPP) $\mathrm{n}$ backbone (Table 1). These peptides were coated at various concentrations onto a glass surface in order to optimize the coating method. After wash of non-bound material, washed human platelets were added to determine the ability of the immobilised peptides to support static platelet adhesion (Fig. 1). In general, the adhesion was higher to $(\mathrm{GPO})_{\mathrm{n}}$ than to $(\mathrm{GPP})_{\mathrm{n}}$ peptides, and it decreased in the order of GFOGER > GLOGER > 
GLSGER > GAOGER, such in agreement with the lower binding affinity for $\alpha_{2} \beta_{1}$. For all peptides, a coating concentration of $500 \mathrm{~g} \mathrm{~mL}^{-1}$ was sufficient for optimal platelet adhesion.

Table 1. Properties of the synthesised triple-helical, collagen-mimetic GXX'GER peptides. Shown are the abbreviated hexapeptide sequences, incorporated into GPO or GPP triple helices; further the number of loci of these sequences in collagen types I, III and IV. IC 50 values are given of replacement of platelet binding to monomeric collagen (13).

\begin{tabular}{|c|c|c|c|}
\hline Abbreviation & Full sequence & $\begin{array}{l}\text { Collagen } \\
\text { loci }\end{array}$ & $\begin{array}{l}\mathrm{IC}_{50} \\
(\mu \mathrm{M})\end{array}$ \\
\hline GFOGER-(GPO)n & $\mathrm{H}-\mathrm{GPC}(\mathrm{GPO})_{3} \mathrm{GFOGER}(\mathrm{GPO})_{3} \mathrm{GPC}-\mathrm{NH}_{2}$ & 5 & 1.3 \\
\hline GFOGER-(GPP) $n$ & $\mathrm{H}-\mathrm{GPC}(\mathrm{GPP})_{5} \mathrm{GFOGER}(\mathrm{GPP})_{5} \mathrm{GPC}-\mathrm{NH}_{2}$ & & 0.2 \\
\hline GLOGER-(GPO)n & $\mathrm{H}-\mathrm{GPC}(\mathrm{GPO})_{3} \mathrm{GLOGER}(\mathrm{GPO})_{3} \mathrm{GPC}-\mathrm{NH}_{2}$ & 3 & 3.9 \\
\hline GLOGER-(GPP) $)_{n}$ & $\mathrm{H}-\mathrm{GPC}(\mathrm{GPP})_{5} \mathrm{GLOGER}(\mathrm{GPP})_{5} \mathrm{GPC}-\mathrm{NH}_{2}$ & & 6.3 \\
\hline GLSGER-(GPO) $n$ & $\mathrm{H}-\mathrm{GPC}(\mathrm{GPO})_{3} \mathrm{GLSGER}(\mathrm{GPO})_{3} \mathrm{GPC}-\mathrm{NH}_{2}$ & 1 & 4.8 \\
\hline GLSGER-(GPP) $)_{n}$ & H-GPC $(\mathrm{GPP})_{5}$ GLSGER(GPP $)_{5}$ GPC-NH ${ }_{2}$ & & 34.6 \\
\hline GMOGER-(GPO) $n$ & $\mathrm{H}-\mathrm{GPC}(\mathrm{GPO})_{3} \mathrm{GMOGER}(\mathrm{GPO})_{3} \mathrm{GPC}-\mathrm{NH}_{2}$ & 2 & 16.5 \\
\hline GMOGER-(GPP) $)_{n}$ & $\mathrm{H}-\mathrm{GPC}(\mathrm{GPP})_{5} \mathrm{GMOGER}(\mathrm{GPP})_{5} \mathrm{GPC}-\mathrm{NH}_{2}$ & & 95.8 \\
\hline GASGER-(GPO) $n$ & $\mathrm{H}-\mathrm{GPC}(\mathrm{GPO})_{3} \mathrm{GASGER}(\mathrm{GPO})_{3} \mathrm{GPC}-\mathrm{NH}_{2}$ & 1 & 109 \\
\hline GASGER-(GPP) $)_{n}$ & $\mathrm{H}-\mathrm{GPC}(\mathrm{GPP})_{5} \mathrm{GASGER}(\mathrm{GPP})_{5} \mathrm{GPC}-\mathrm{NH}_{2}$ & & $>200$ \\
\hline GAOGER-(GPO) $n$ & $\mathrm{H}-\mathrm{GPC}(\mathrm{GPO})_{3} \mathrm{GAOGER}(\mathrm{GPO})_{3} \mathrm{GPC}-\mathrm{NH}_{2}$ & 1 & 153 \\
\hline GAOGER-(GPP) $)_{n}$ & $\mathrm{H}-\mathrm{GPC}(\mathrm{GPP})_{5} \mathrm{GAOGER}(\mathrm{GPP})_{5} \mathrm{GPC}-\mathrm{NH}_{2}$ & & $>200$ \\
\hline
\end{tabular}

To determine the coating efficiency, glass coverslips containing multiple spots of two representative peptides, i.e. GLSGER-(GPO) ${ }_{n}$ and GAOGER-(GPP) ${ }_{n}$ (coating concentration $500 \mu \mathrm{g} \mathrm{mL}^{-1}$ ), were analysed for actually bound material. Extraction of the immobilized peptides required a treatment with chaotropic agent, $6 \mathrm{~mol} \mathrm{~L}^{-1}$ guanidine in acetate buffer. The extracted material was reduced with $ß$-mercaptoethanol, and applied to reversedphase HPLC. As shown in Fig. 2A, the eluted GLSGER-(GPO)n appeared as a double peak at 25-26\% acetonitrile, representing its reduced form (peak 1) and a ß-mercaptoethanol adduct (peak 2). ESI-MS showed that the eluted peptide had a molecular mass of 2718.6 Da (Fig. 2A), which approximated the theoretical mono-isotopic mass (2717.2 Da) and the average mass (2719.0 Da). Using the same procedure, the recovered GAOGER-(GPP) $n$ peptide eluted in HPLC as one major peak at $34 \%$ acetonitrile, which was identified as the reduced peptide and ß-mercaptoethanol adduct (Fig. 2B, peak 3). The observed molecular mass was 3643.8 Da, which fitted well between the theoretical mono-isotopic mass (3641.7 $\mathrm{Da}$ ) and the average mass (3644.2 Da). The mass spectrum showed an additional $\mathrm{m} / \mathrm{z}$ value 
of 1240.8 , representing the triple protonated state of the ß-mercaptoethanol adduct of GAOGER-(GPP) .

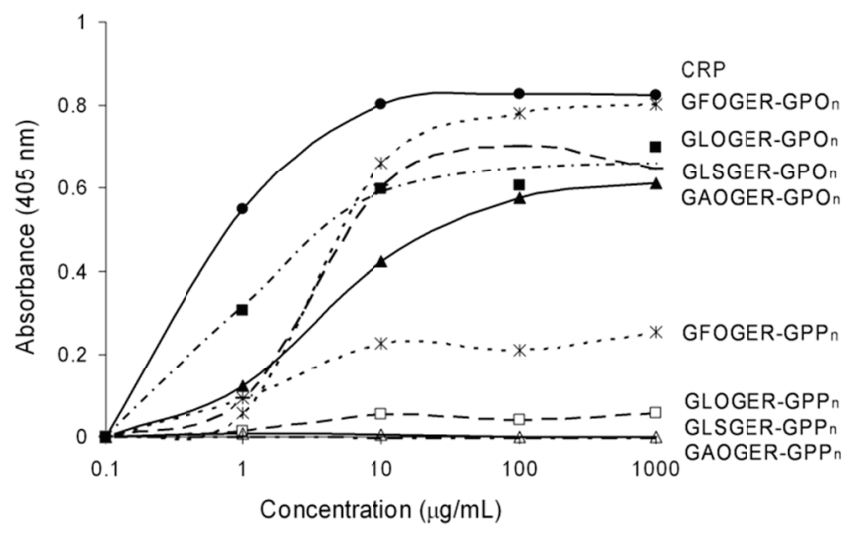

Figure 1. Effect of coating density of collagen-mimetic peptides on platelet adhesion. Glass coverslips were coated with solutions of $0.1-1000 \mu \mathrm{g} \mathrm{L}^{-1}$ of GXX'GER-(GPO) ${ }_{n}$ or GXX'GER-(GPP) ${ }_{n}$ peptide, as indicated. After wash of all unbound peptide, static adhesion of washed platelets ( 1 h) was determined colorimetrically (absorbance $405 \mathrm{~nm}$ ). Data are means \pm SEM $(n=3)$.

By calibrating with pure reduced peptides, peak areas from the HPLC profiles allowed semi-quantitative determination of the recovery of the peptides from glass coverslips. For GLSGER-(GPO) $n$ and GAOGER-(GPP) $n$ the mean recovery was about $20 \%$ ( $n=7-10$ spots), corresponding to $\sim 1.1 \mu \mathrm{g} / \mathrm{sppot}$ or a density of $\sim 70 \mathrm{ng} / \mathrm{mm}^{2}$. Since the peptides could only be eluted from coverslips by guanidine and not by acetate buffer alone, this pointed to a firm and high-density coating to the glass surface.

Coated triple-helical GFOGER-(GPO) ${ }_{n}$ peptide with vWF mediates thrombus formation at high shear rate

The coated triple-helical GXX'GER peptides were used to study platelet adhesion and thrombus formation under high-shear flow conditions, using a validated experimental setup $(5,7,10)$. Human blood was anticoagulated with PPACK, to completely prevent fibrin formation, and was perfused over the various surfaces at a moderately high shear rate of 1000 $\mathrm{s}^{-1}$. Coverslips coated with native collagen types I and III were used as reference surfaces. Flow over collagen type I and III resulted in rapid platelet adhesion and aggregate formation (Fig. 3A a, b). Staining for PS-exposing platelets (FITC-annexin $A_{5}$ ) or for platelets expressing P-selectin (FITC anti-CD62 mAb) gave many single platelets which exposed PS and aggregates of $\mathrm{P}$-selectin positive platelets. This heterogeneity in platelet activation in flowdependent thrombus formation has been described elsewhere (26). In the absence of coating, no platelets adhered, as reported before (5). 

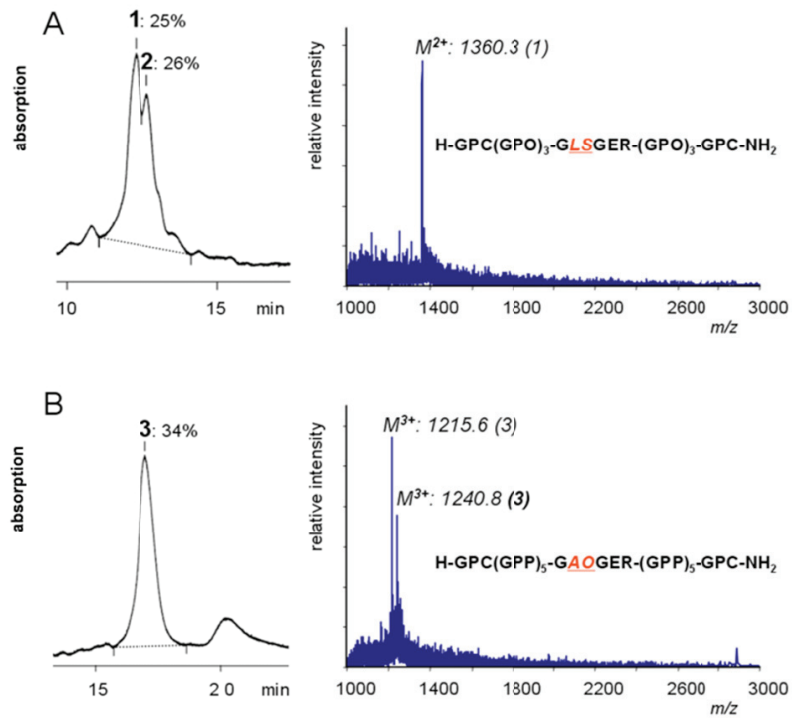

Figure 2. Recovery and analysis of coated GLSGER-(GPO $)_{n}$ and GAOGER-(GPP) ${ }_{n}$ peptides by HPLC and ESI-MS. Coated GLSGER-(GPO) $)_{n}(A)$ and GAOGER-(GPP) $)_{n}(B)$ peptides were extracted from glass coverslips, and analysed using HPLC (left panels) and ESI-MS (right panels). Coating was at $500 \mathrm{\mu g} \mathrm{mL}^{-1}$, followed by wash. ESI-MS showed a double protonated state of reduced GLSGER-(GPO) $(1)$ and a triple protonated state of reduced GAOGER-(GPP) $(3)$, correlating well with the theoretical peptide masses of 2718.2 and $3641.7 \mathrm{Da}$, respectively. When calibrated against pure reduced peptides by HPLC, coating efficiencies on coverslips were determined at about $20 \%$ for GLSGER-(GPO) $n$ and GAOGER$(\mathrm{GPP})_{\mathrm{n}}$.

As VWF is required for collagen-dependent platelet adhesion under shear (27), coverslips were also coated with $v W F$, alone or in combination with triple-helical peptides. Staining of the coated coverslips with anti-vWF mAb showed 5 -fold (co-coating with peptide and VWF) or 10-fold (coating with vWF alone) increased signal intensities in comparison to uncoated coverslips. This points to adequate immobilisation of VWF on the glass surface. Perfusion of blood (1000 $\mathrm{s}^{-1}$ ) over coverslips coated with only vWF resulted in adhesion of mostly single platelets with limited spreading (Fig. 3A C), such in agreement with published data $(27,28)$. In contrast, using GFOGER- $(\text { GPO })_{n}$ as coating, platelets stably adhered and formed compact aggregates (Fig. 3A d). Co-coating of GFOGER-(GPO) $)_{n}$ and vWF resulted in increased adhesion of platelets, which again formed aggregates (Fig. $3 \mathrm{~A}$ e). Staining of activated platelets on coverslips with GFOGER-(GPO) $n \pm v W F$ showed clusters of single PSexposing platelets and aggregates of P-selectin positive platelets (Fig. 3A c-e).

The adhesion of platelets on GFOGER-(GPO) was linear in time, up to a period of 4 min, which is consistent with previous observations for collagen type I surface (7). At this time point, confocal image stacks showed that thrombi of calcein-labelled platelets had an average volume of 140 and $110 \mu \mathrm{m}^{3}$ per $1000 \mu \mathrm{m}^{3}$ on coated GFOGER-(GPO) $n$ and collagen I, respectively. 
Quantitative analysis of platelet deposition was performed at the 4-min end point, by recording phase-contrast and fluorescence images from the coated surfaces. Single platelets on collagen, but not on VWF alone, did not bind FITC-annexin A5 (Fig. 3B). The platelet aggregates formed on immobilised GFOGER-(GPO) $n$ with(out) vWF occupied a surface area of $23-27 \%$ and were somewhat reduced in PS exposure in comparison to platelets on collagen. The activated state of platelets on coverslips coated with GFOGER-(GPO) $/$ VWF was confirmed by measuring single-cell $\mathrm{Ca}^{2+}$ fluxes, which showed strong and prolonged increases in cytosolic $\mathrm{Ca}^{2+}$, similar to those in platelets on type I collagen (I. Munnix, unpublished data, 2008).

A
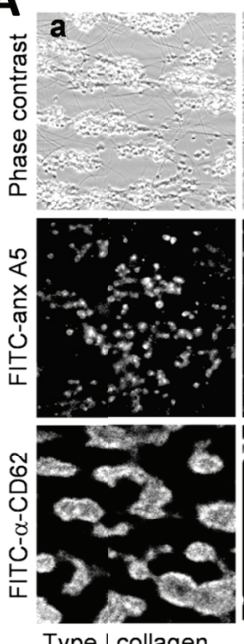

Type $\mid$ collagen
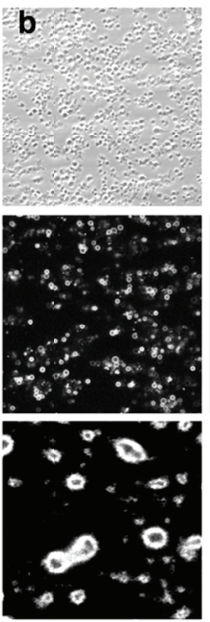

Type III collagen
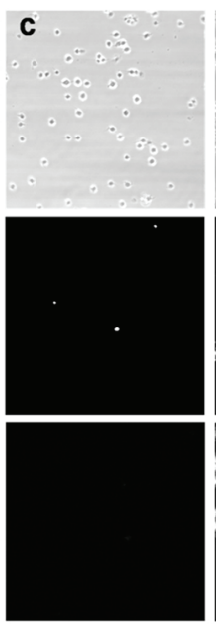

VWF
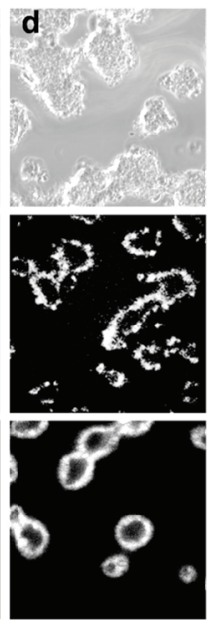

GFOGER-(GPO)r
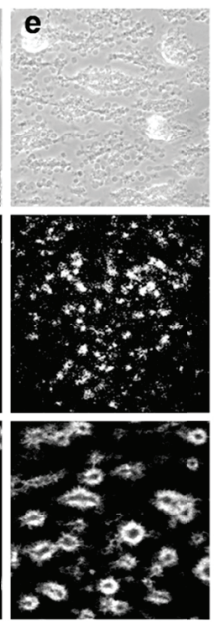

VWF +

GFOGER-(GPO)n

B

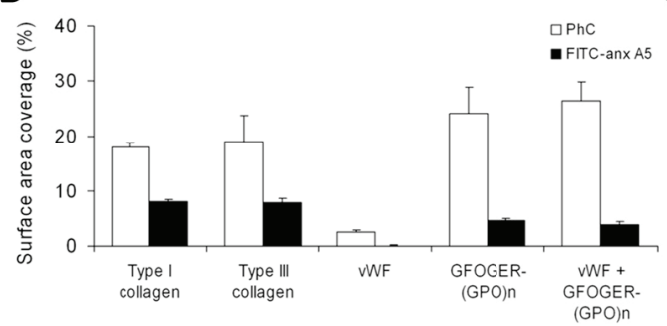

C

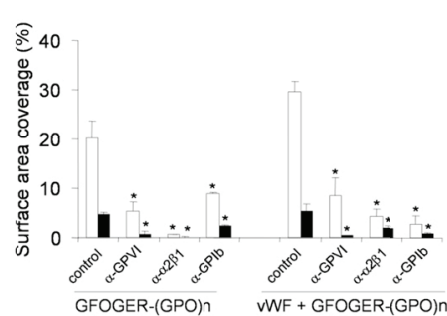

Figure 3. Flow-induced thrombus formation on collagen or coated GFOGER-(GPO) in the presence or absence of vWF. Coverslips were coated with collagen type I (a), type III (b), vWF (c), GFOGER$(G P O)_{n}$ alone (d) or with VWF (e). Blood was flowed over these coatings for 4 min at $1000 \mathrm{~s}^{-1}$, followed by stain with FITC-annexin $A_{5}$ or FITC- $\alpha-C_{2} 62$ mAb. (A) Representative phase-contrast and fluorescence images after perfusion. (B) Platelet deposition and PS exposure. (C) Effects of inhibiting antibodies: scFv $10 B_{12}$ to block GPVI ( $\left.\alpha-G P V I, 100 \mu \mathrm{gL}^{-1}\right),{ }_{15} \mathrm{D}_{7} \mathrm{mAb}$ to block $\alpha_{2} \beta_{1}\left(\alpha-\alpha_{2} \beta_{1}, 20 \mu \mathrm{gL}^{-1}\right)$ or $6 \mathrm{~B}_{4}$ to block GPIb ( $\alpha-\mathrm{GPIb}, 20 \mu \mathrm{g} \mathrm{mL}^{-1}$ ). Bars indicate coveragle of all platelets (white) and annexin $\mathrm{A}_{5}$ binding platelets (black). Mean \pm SEM $(n=6-8), * p<0.05$ compared to vehicle control. 
To dissect specific involvement of the collagen and vWF receptors in platelet adhesion to the coated peptide surfaces, blood was preincubated with maximally effective concentrations of blocking mAbs against GPVI, $\alpha_{2} \beta_{1}$ or GPIb. Inhibition of GPVI with scFv $10 B_{12}$ (5) permitted stable adherence of mostly single platelets to coated GFOGER-(GPO) $n \pm v W F$, but large platelet aggregates and PS-exposing platelets were no longer present (Fig. ${ }_{3} \mathrm{C}$ ). Blocking of $\alpha_{2} \beta_{1}$ integrin by ${ }_{15} \mathrm{D}_{7} \mathrm{mAb}$ (9) markedly reduced the area coverage and PS exposure of platelets on GFOGER-(GPO) \pm vWF. When GPIb $\alpha$ was inhibited by $6 \mathrm{~B}_{4} \mathrm{mAb}$ (21), area coverage of platelets again markedly decreased on GFOGER-(GPO)n/VWF, and to a lesser degree on GFOGER-(GPO) alone. Together, these results indicate that immobilised triple-helical GFOGER-(GPO) $n$ peptide in combination with vWF effectively mimics native type I collagen in mediating $\alpha_{2} \beta_{1-}$, GPVI- and GPIb-dependent platelet adhesion and aggregation under flow.

Coated triple-helical peptides with either GFOGER or (GPO) ${ }_{n}$ sequences require vWF for full thrombus formation at high shear rate

To distinguish between the contribution of the $\alpha_{2} \beta_{1}$ - and GPVI-binding motifs of GFOGER$(\mathrm{GPO})_{n}$, homologous peptides were used that contained only one of these motifs. Coverslips were coated with $C R P$, i.e. the classical triple-helical $(G P O)_{n}$ peptide which activates platelets exclusively via GPVI. At $1000 \mathrm{~s}^{-1}$ platelets did not adhere to coated CRP, similarly as to VWF (Fig. 4A a-b). In contrast, on coverslips coated with CRP plus VWF, platelets adhered well and formed aggregates, although these were smaller in size than those on GFOGER$(\mathrm{GPO})_{n} /$ VWF surface (Fig. 4A c). Staining with FITC-annexin $\mathrm{A}_{5}$ showed many PS-exposing platelets on CRP/VWF, while again platelet aggregates were $P$-selectin positive (Fig. $4 A, B$ ).

Flow experiments performed at a higher shear rate of $1700 \mathrm{~s}^{-1}$, representative for that in middle-sized arteries, indicated formation of single platelets and micro-aggregates on coated vWF alone (area coverage $<2 \%$ ). Further, increase in shear rate from 1000 to $1700 \mathrm{~s}^{-1}$ increased platelet deposition and aggregation on coated CRP/VWF (area coverage from $19.2 \pm 3.9$ to $47.6 \pm 1.1 \%$ ) and on coated GFOGER-(GPO) $/$ VWF (area coverage from $26.5 \pm 3.4$ to $47.3 \pm 1.2 \%$ ), thus underlining the cooperative activity of poly-GPO peptides with vWF. 
A

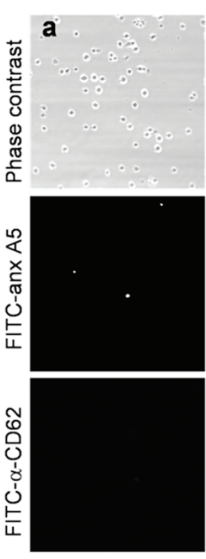

vWF
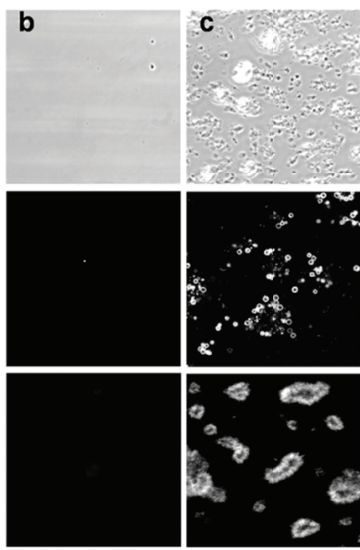

CRP
B

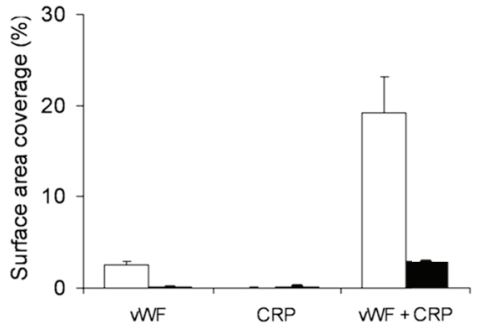

Figure 4. Flow-induced thrombus formation on coated CRP in the presence or absence of vWF. Blood was flowed for 4-min at $1000 \mathrm{~s}^{-1}$ using coverslips coated with vWF (a), CRP (b) or combination of both (c). Staining was with FITC-annexin A5 or FITC- $\alpha-C D 62$ mAb. (A) Representative phase-contrast and fluorescence microscopic images after perfusion. (B) Surface area coverage of all platelets (white bars) and annexin A5-binding platelets (black bars) for each surface type $(n=6-8)$.

Complementary experiments were performed using coated GFOGER-(GPP) n $_{n}$ peptide in which the GPVI-activating GPO stretches were replaced by inactive GPP triplets. At high shear, platelets did adhere to GFOGER-(GPP) ${ }_{n}$ but formed only small aggregates (Fig. $5 \mathrm{~A}$ a). However, large aggregates were formed on coverslips co-coated with GFOGER-(GPP) $/$ /vWF (Fig. $5 \mathrm{~A} \mathrm{~b}$ ). These aggregates were typically loose in structure and disintegrated upon prolonged rinse with Hepes buffer. Platelets which adhered to GFOGER-(GPP) \pm vWF showed little, but still detectable staining with FITC-annexin A 5 (Fig. ${ }_{5} \mathrm{~B}$ ).

On GFOGER-(GPP)n/vWF surface, blocking of GPVI with scFv $10 \mathrm{~B} 12$ substantially reduced platelet deposition, aggregate formation and, interestingly, PS exposure (Fig. $5 \mathrm{~A}$ c). Blocking of $\alpha_{2} \beta_{1}$ (15 77 mAb) had similar effects (Fig. 5A, d), but blocking of GPIb (6B4 $\mathrm{mAb}$ ) did not affect PS exposure (Fig. 5A, B). Furthermore, GPVI blocking reduced numbers of annexin A5-binding platelets on GFOGER-(GPP) $n$ surface. Together, this suggests residual involvement of GPVI next to $\alpha_{2} \beta_{1}$ in platelet aggregation and PS exposure on coated GFOGER-(GPP) $)_{n}$. 


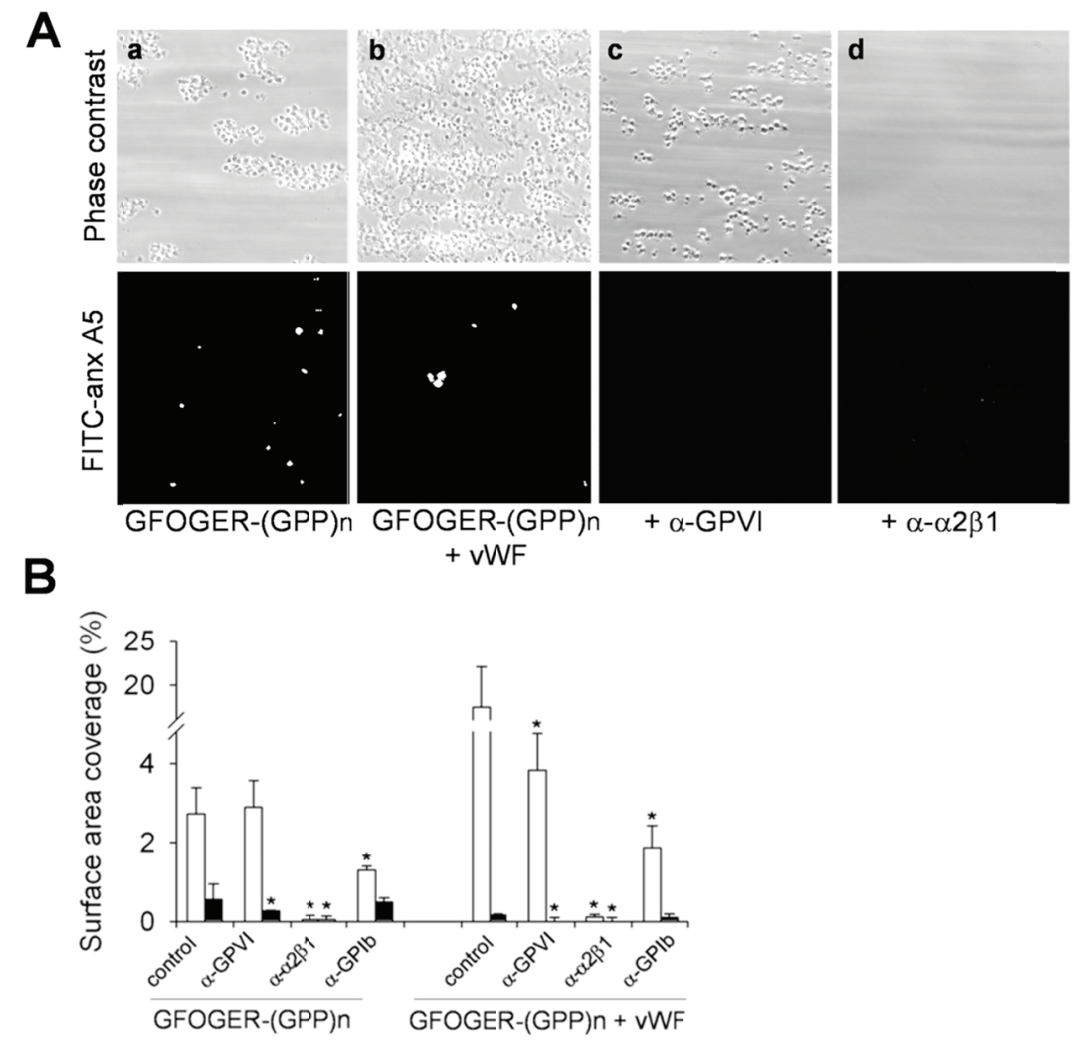

Figure 5. Flow-induced thrombus formation on GFOGER-(GPP) $n$ surfaces in the presence or absence of vWF. Blood was flowed for 4-min at $1000 \mathrm{~s}^{-1}$ over coverslips coated with GFOGER-(GPP) $n$ alone or in combination with vWF. (A) Representative phase-contrast and fluorescence images after flow in the presence of (a-b) vehicle, (c) $100 \mu \mathrm{g} \mathrm{mL}^{-1} \mathrm{scFv} 10 \mathrm{~B} 12$ to block GPVI ( $\alpha-\mathrm{GPVI}$ ), or (d) $20 \mu \mathrm{g} \mathrm{mL}^{-1} 15 \mathrm{D} 7 \mathrm{mAb}$ to block $\alpha_{2} \beta_{1}\left(\alpha-\alpha_{2} \beta_{1}\right)$. (B) Effect of the blocking antibodies including $6 B_{4}(\alpha-G P I b)\left(20 \mu g \mathrm{~mL}^{-1}\right)$ on platelet deposition. Bars show percentages of surface area coverage of all platelets (white) and annexin A5-binding platelets (black) $(n=3-5, * p<0.05$ compared to vehicle control).

Coated lower- $\alpha_{2} b_{1}$ affinity $G X X^{\prime} G E R-(G P O)_{n}$ and $G X X^{\prime} G E R-(G P P)_{n}$ peptides differ in potency to induce thrombus formation at high shear rate

To determine how modulation of integrin $\alpha_{2} \beta_{1}$ binding affects the adhesive properties of the triple-helical peptides, lower affinity homologues of GFOGER-(GPO) $n$ and GFOGER$(G P P)_{n}$ were used. As indicated in Table 1, triple-helical peptides containing a GLOGER, GLSGER or GMOGER sequence, have a reduced, moderate affinity for $\alpha_{2} \beta_{1}$. Furthermore, peptides with a GASGER or GAOGER sequence are low in affinity for the integrin (IC50 > 100 $\mu \mathrm{M})(13)$.

Unlike GFOGER-(GPO) and GFOGER-(GPP) $)_{n}$ coated peptides with low-affinity GASGER or GAOGER motifs, in either a $(\mathrm{GPO})_{n}$ or a (GPP) ${ }_{n}$ backbone, were unable to support platelet adhesion, aggregation or PS exposure under shear conditions (Fig. 6A, B). However, when coated together with vWF, the GASGER and GAOGER peptides supported 
platelet adhesion particularly when incorporated into (GPO) ${ }_{n}$ sequences (Fig. 6C). Staining with FITC-annexin $A_{5}$ indicated significant PS exposure when both vWF and $(G P O)_{n}$ were present. Together, these results suggest that peptides with low affinity $\alpha_{2} \beta_{1}$-binding motifs (GASGER and GAOGER) are highly platelet adhesive only in combination with binding sites for GPVI (poly-GPO) and GPIb (vWF).
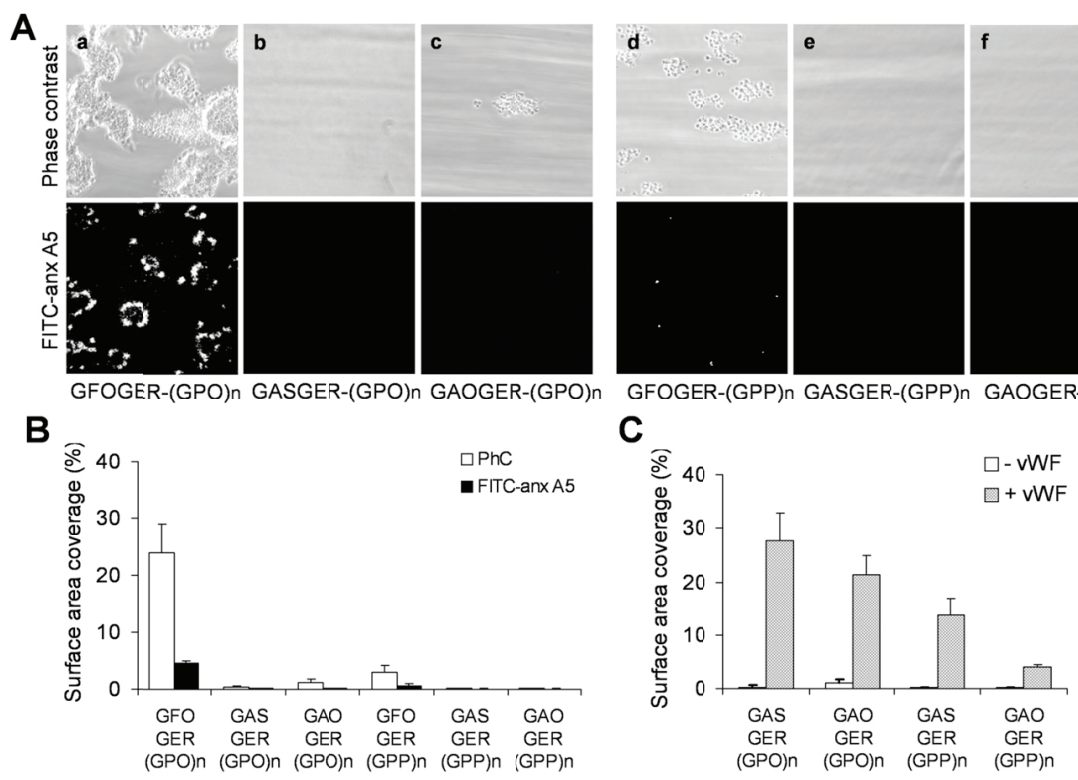

Figure 6. Flow-induced thrombus formation on surfaces of various $\alpha_{2} \beta_{1}$-binding triple helical peptides. Blood was flowed over coverslips with coated $G X X^{\prime} G E R-(G P O)_{n}$ or GXX'GER-(GPP) $n$ and vWF, as indicated. (A) Representative phase-contrast and fluorescence images are given after perfusion over indicated coatings for $4 \mathrm{~min}$ at $1000 \mathrm{~s}^{-1}$. (B) Surface area coverage of all platelets (white bars) and PSexposing platelets (black bars) with each type of surface. (C) Effect of co-coated vWF on surface area coverage of all platelets in the absence (white bars) or presence (crossed bars) of vWF $(n=3-7)$.

Coated $G X X^{\prime} G E R-(G P O)_{n}$ and $G X X^{\prime} G E R-(G P P)_{n}$ peptides differ in platelet adhesion and aggregation at low shear rate

The platelet-adhesive potency of the triple-helical peptides was also studied under low shear conditions, where VWF and GPIb do not play a role. On coverslips with GFOGER$(G P O)_{n}$ few large aggregates with annexin A5-binding platelets were formed (Fig. 7A). In contrast, on GFOGER-(GPP) $n$ only single platelets and small aggregates were formed, although some platelets stained with annexin $\mathrm{A}_{5}$. Coated CRP was moderately effective. For the other $(G P O)_{n}$ peptides, the potency of platelet adhesion decreased in the order of GFOGER, GLOGER > GMOGER, GAOGER, GLSGER > GASGER (Fig. 7B). Annexin A5 staining also followed this order. Interestingly, the low-affinity $\alpha_{2} \beta_{1}$ binding peptide GAOGER$(G P O)_{n}$ supported platelet adhesion/aggregation and PS exposure better than its homologue GASGER-(GPO) . For the (GPP) $)_{n}$ containing peptides, adhesion was in the order of predicted $\alpha_{2} \beta_{1}$ affinities: GFOGER > GLOGER > GMOGER, GLSGER, GASGER, GAOGER 
(Fig. 7B). Annexin A5 staining was negligible. Together, the data show that, at low shear rate, only the combination of GFOGER and (GPO) $)_{n}$-but not (GPP) $)_{n}-$ motifs leads to formation of aggregates with procoagulant platelets.
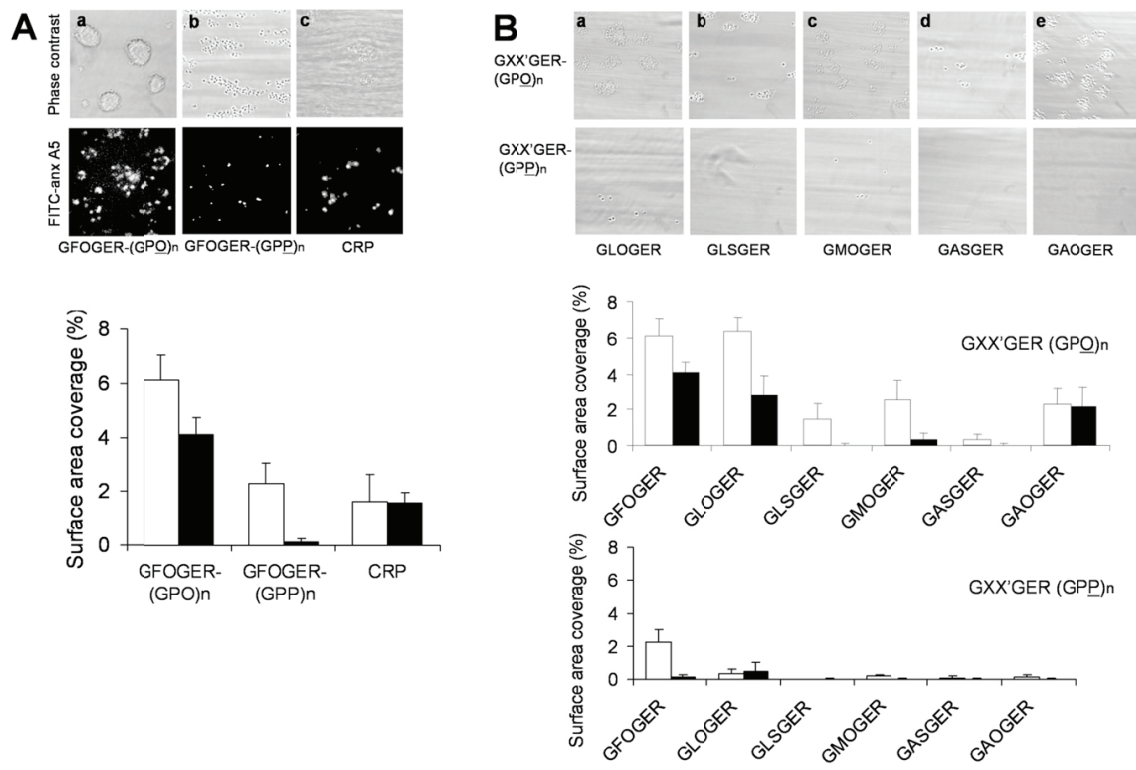

Figure 7. Flow-induced thrombus formation on surfaces of various $\alpha_{2} \beta_{1}$-binding triple helical peptides at low shear rate. Blood was flowed for 6 min at $150 \mathrm{~s}^{-1}$ over indicated coatings. (A) Representative phase-contrast and fluorescence images of coverslips coated with (a) GFOGER-(GPO)n, (b) GFOGER-(GPP) , or (c) CRP. (B) Representative phase-contrast and fluorescence images of coverslips coated with GXX'GER-(GPO) $)_{n}$ or GXX'GER-(GPP) $)_{n}$ peptides. Bars give surface area coverage of all platelets (white) and annexin $A_{5}$ binding platelets (black) per type of surface $(n=3-5)$.

\section{Discussion}

The presented findings indicate that collagen-mimetic, triple-helical peptides, immobilised to glass, effectively and efficiently can take over the functions of native collagens in mediating platelet adhesion and activation via the receptors GPVI and $\alpha_{2} \beta_{1}$. At high shear rate and with vWF present, peptides with the GPVI-binding $(\mathrm{GPO})_{\mathrm{n}}$ sequence or the high-affinity $\alpha_{2} \beta_{1}$-binding GFOGER motif caused stable adhesion, activation and aggregation of platelets. Adhesion and aggregation were sensitive to blockade of the VWF receptor GPIb. In the absence of $\mathrm{VWF}$, the combination of GFOGER and (GPO) $n$ sequences was required for platelet adhesion and activation. In general, on $(\mathrm{GPO})_{\mathrm{n}}$ containing surfaces, including CRP, platelet activation was detected as P-selectin expression and exposure of PS, measured by binding of FITC-annexin $A_{5}$. The GFOGER sequence has been identified as the major highaffinity $\alpha_{2} \beta_{1}$-binding stretch in type I collagen (13) but not type III collagen (29), which given the present results implicates that it is readily exposed for platelet adhesion in native type I collagen fibers. 
The integrin-binding peptides used in this study consisted of one copy of high-affinity (GF/LOGER), intermediate affinity (GLO/SGER) or low-affinity (GAO/SGER) $\alpha_{2} \beta_{1}$ recognition site within the backbone of repeated GPP or GPO sequences to provide triple-helical conformation $(13,30)$. The GER part functions to recruit platelets via $\alpha_{2} \beta_{1}$, and the GPO part acts to activate GPVI. The peptides were synthesised based on the assumption that the integrin-binding motif spans a 6 amino acid sequence, as demonstrated in the co-crystal model of $\alpha_{2}$ I-domain and GFOGER (19). Surprisingly, when immobilised on a surface, especially the high-affinity GFOGER peptides are active in trapping platelets and forming aggregates under flow. At high-shear conditions they synergise with GPIb-vWF interactions. In addition, combination of the $\alpha_{2} \beta_{1}$-binding domain and $(G P O)_{n}$ sequences markedly increases platelet adhesion and activation via GPVI under flow.

When comparing the various triple-helical peptides, the most potent surface is provided by GFOGER-(GPO) $)_{n}$, which mediates platelet adhesion and aggregate formation via $\alpha_{2} \beta_{1}$ as well as GPVI. Platelet adhesion to this peptide leads to full GPVI-induced platelet responses, including secretion (P-selectin exposure) and procoagulant activity (PS exposure), both of which rely on stimulation of the LAT/phospholipase $C_{\gamma 2}$ activation pathway $(7,26)$.

Co-coating with vWF further stimulated adhesion and increased platelet accumulation into aggregates. Experiments with blocking antibodies confirmed the involvement of $\alpha_{2} \beta_{1}$, GPVI and GPIb in platelet adhesion to the peptide surfaces, but also supported the concept that interplay between these adhesive receptors is required for stable platelet adhesion and activation under high shear conditions (3-6). An interesting observation is that the previously observed heterogeneity of platelet aggregates, containing discrete populations of aggregated (P-selectin positive) and PS-exposing platelets (26) is not restricted to thrombus formation on native collagen fibres, but also extends to immobilised GFOGER-(GPO) $n$, as, in aggregates, only platelets which were in direct contact with the coated peptide, bound annexin $\mathrm{A}_{5}$.

At high shear rate, CRP, which contains just $(G P O)_{n}$ sequences, mediated platelet adhesion and thrombus formation only when co-immobilised with VWF. The same was true for $(G P O)_{n}$-containing peptides with low-affinity $\alpha_{2} \beta_{1}$ binding motifs, GAOGER and GASGER. These results confirm and extend earlier evidence that combined adhesion via GPIb and GPVI is sufficient to induce thrombus formation, not only on native collagen (5), but also on synthetic peptides (this chapter).

An unexpected finding was that the $\alpha_{2} \beta_{1}$-binding GFOGER-(GPP) neptide, lacking GPVI-activating (GPO) $)_{n}$ stretches, caused low, though readily-detectable platelet aggregation and PS exposure. This was clear in both high- and low-shear experiments. This is in good agreement with the earlier determined high binding affinity to $\alpha_{2} \beta_{1}$ on platelets (Table 1). Since these platelet responses are suppressed by blocking GPVI with scFv 10B12, it was concluded that the GFOGER-(GPP) ${ }_{n}$ surface has limited GPVI-dependent activity. This is not without precedence, as others have shown that static adhesion of platelets to the GFOGER peptide results in tyrosine phosphorylation of proteins of the GPVI-FCR $\gamma$-chain cascade, including Src, Syk, SLP76 and phospholipase $C \gamma_{2}$ (11). The authors suggested that 
$\alpha_{2} \beta_{1}$ engagement via outside-in signaling leads to a similar activation pathway as GPVI ligation. Effects of GPVI-blocking agents were not determined in this study, but it was demonstrated that platelets deficient in GPVI/FcR spread normally on GFOGER-(GPP) ${ }_{n}$ while phospholipase $C_{\gamma} \gamma_{2}$ deficient platelets showed impaired spreading. It is possible that the GFOGER peptide signals differently under static and flow conditions. However, one possibility is that $\alpha_{2} \beta_{1}$ engagement with GFOGER does lead to activation (clustering?) of GPVI, as these receptors have been shown to interact during collagen activation of platelets (3). This needs further investigation.

The results of low-shear experiments further point to marked synergism between GPVI (GPO) and high-affinity $\alpha_{2} \beta_{1}$ binding (GFOGER) in adhesion and aggregation, but not between GPVI and low-affinity $\alpha_{2} \beta_{1}$ binding. In other words, the low-affinity $\alpha_{2} \beta_{1}$ GAO/SGER peptides were poorly adhesive within a (GPO) $)_{n}$ backbone, and not at all active if presented within a (GPP) ${ }_{n}$ backbone unless VWF was also present. It is known that type I and III collagens contain more low-affinity than high-affinity $\alpha_{2} \beta_{1}$ binding sites $(13,29)$. Hence, the current study with model peptides would predict that, in platelet interaction with native collagens, especially the high-affinity integrin binding sites contribute to shear-dependent thrombus formation.

\section{Acknowledgements}

We thank J. Fletcher and N. Pugh for assistance. This work was on a Marie-Curie EST 2005020706-3 grant from the European Commission (KG). Support was from the UK Medical Research Council, the British Heart Foundation, the Netherlands Organization for Scientific Research (114000076) and the Academy of Finland.

\section{References}

1 Savage B, Shattil SJ, \& Ruggeri ZM. Modulation of platelet function through adhesion receptors. A dual role for glycoprotein IIb/IIla mediated by fibrinogen and glycoprotein Ilb-von Willebrand factor. J Biol Chem 267, 11300-6 (1992).

2 Nieswandt B \& Watson SP. Platelet-collagen interaction: is GPVI the central receptor? Blood 102, 449-61 (2003).

3 Chen $\mathrm{H}$ \& Kahn ML. Reciprocal signaling by integrin and nonintegrin receptors during collagen activation of platelets. Mol Cell Biol 23, 4764-77 (2003).

4 Atkinson BT, Jarvis GE, \& Watson SP. Activation of GPVI by collagen is regulated by $\alpha_{2} \beta_{1}$ and secondary mediators. J Thromb Haemost 1, 1278-87 (2003).

5 Siljander PR, Munnix IC, Smethurst PA, Deckmyn H, Lindhout T, Ouwehand WH, Farndale RW, \& Heemskerk JW. Platelet receptor interplay regulates collagen-induced thrombus formation in flowing human blood. Blood 103, 1333-41 (2004).

6 Auger JM, Kuijpers MJ, Senis YA, Watson SP, \& Heemskerk JW. Adhesion of human and mouse platelets to collagen under shear: a unifying model. FASEB J 19, 825-7 (2005).

7 Lecut C, Schoolmeester A, Kuijpers MJ, Broers JL, van Zandvoort MA, Vanhoorelbeke K,

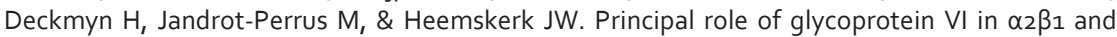
$\alpha \mathrm{llb} \beta_{3}$ activation during collagen-induced thrombus formation. Arterioscler Thromb Vasc Biol 24, 1727-33 (2004).

8 Cosemans JM, Munnix IC, Wetzker R, Heller R, Jackson SP, \& Heemskerk JW. Continuous signaling via phosphoinositide 3 -kinase isoforms $\beta$ and $\gamma$ is required for platelet ADP receptor function in dynamic thrombus stabilization. Blood 108, 3045-52 (2006). 

Nurden A, Vanhoorelbeke $K$, \& Deckmyn $H$. Activation of $\alpha$ llb $\beta_{3}$ is sufficient but also an imperative prerequisite to activate a2b1 on platelets. Blood 109, 595-602 (2007).

10 Munnix IC, Strehl A, Kuijpers MJ, Auger JM, van der Meijden PE, van Zandvoort MA, oude Egbrink M, Nieswandt B, \& Heemskerk JW. The glycoprotein VI-phospholipase C $C_{2}$ signaling pathway controls thrombus formation induced by collagen and tissue factor in vitro and in vivo. Arterioscler Thromb Vasc Biol 25, 2673-8 (2005).

11 Inoue O, Suzuki-Inoue K, Dean WL, Frampton J, \& Watson SP. Integrin $\alpha_{2} \beta_{1}$ mediates outside-in regulation of platelet spreading on collagen through activation of Src kinases and PLCY2. J Cell Biol 160, 769-80 (2003).

12 Nieswandt B, Brakebusch C, Bergmeier W, Schulte V, Bouvard D, Mohtari-Nejad R, Lindhout T, Heemskerk JW, Zirngibl H, \& Fässler R. Glycoprotein VI but not $\alpha_{2} \beta_{1}$ integrin is essential for platelet interaction with collagen. EMBO J 20, 2120-30 (2001).

13 Siljander PR, Hamaia S, Peachey AR, Slatter DA, Smethurst PA, Ouwehand WH, Knight CG, \& Farndale RW. Integrin activation state determines selectivity for novel recognition sites in fibrillar collagens. J Biol Chem 279, 47763-72 (2004).

14 Kehrel B, Wierwille S, Clemetson KJ, Anders O, Steiner M, Knight CG, Farndale RW, Okuma M, \& Barnes MJ. Glycoprotein VI is a major collagen receptor for platelet activation: it recognizes the platelet-activating quaternary structure of collagen, whereas $\mathrm{CD}_{3} 6$, glycoprotein IIb/IIla, and von Willebrand factor do not. Blood 91, 491-9 (1998).

15 Farndale RW, Sixma JJ, Barnes MJ, \& de Groot PG. The role of collagen in thrombosis and haemostasis. J Thromb Haemost 2, 561-73 (2004).

16 Smethurst PA, Onley DJ, Jarvis GE, O'Connor MN, Knight CG, Herr AB, Ouwehand WH, \& Farndale RW. Structural basis for the platelet-collage interaction. The smallest motif within collagen that recognizes and activates platelet glycoprotein $\mathrm{VI}$ contains to glycine-prolinehydroxyproline triplets. J Biol Chem 282, 1296-304 (2007).

17 Xu Y, Gurusiddappa S, Rich RL, Owens RT, Keene DR, Mayne R, Hook A, \& Hook M. Multiple binding sites in collagen type I for the integrins $\alpha_{1} \beta_{1}$ and $\alpha_{2} \beta_{1}$. J Biol Chem 275, 35981-9 (2000).

18 Knight CG, Morton LF, Peachey AR, Tuckwell DS, Farndale RW, \& Barnes MJ. The collagenbinding $A$-domains of integrins $\alpha_{1} \beta_{1}$ and $\alpha_{2} \beta_{1}$ recognize the same specific amino acid sequence, GFOGER, in native (triple-helical) collagens. J Biol Chem 275, 35-40 (2000).

19 Emsley J, Knight CG, Farndale RW, Barnes MJ, \& Liddington RC. Structural basis of collagen recognition by integrin $\alpha_{2} \beta_{1}$. Cell 101, 47-56 (2000).

20 Smethurst PA, Joutsi-Korhonen L, O'Connor MN, Wilson E, Jennings NS, Garner SF, Zhang $Y$, Knight CG, Dafforn TR, Buckle A, IJsseldijk MJ, de Groot PG, Watkins NA, Farndale RW, \& Ouwehand $\mathrm{WH}$. Identification of the primary collagen-binding surface on human glycoprotein $\mathrm{VI}$ by site-directed mutagenesis and by a blocking phage antibody. Blood 103, 903-11 (2004).

21 Cauwenberghs N, Vanhoorelbeke K, Vauterin S, Westra DF, Romo G, Huizinga EG, Lopez JA, Berndt MC, Harsfalvi J, \& Deckmyn H. Epitope mapping of inhibitory antibodies against platelet glycoprotein Ib $\alpha$ reveals interaction between the leucine-rich repeat $\mathrm{N}$-terminal and C-terminal flanking domains of glycoprotein Iba. Blood 98, 652-60 (2001).

22 Morton LF, Hargreaves PG, Farndale RW, Young RD, \& Barnes MJ. Integrin $\alpha_{2} \beta_{1}$-independent activation of platelets by simple collagen-like peptides, collagen tertiary (triple-helical) and quaternary (polymeric) structures are sufficient alone for $\alpha_{2} \beta_{1}$-independent platelet reactivity. Biochem J 306, 337-44 (1995).

23 Knight CG, Onley CM, \& Farndale RW. Peptide synthesis in the study of collagen-platelet interact tions. Methods Mol Biol 273, 349-64 (2004).

24 Heemskerk JW, Vuist WM, Feijge MA, Reutelingsperger CP, \& Lindhout T. Collagen but not fibrinogen surfaces induce bleb formation, exposure of phosphatidylserine and procoagulant activity of adherent platelets. Blood 90, 2615-25 (1997).

25 Onley DJ, Knight CG, Tuckwell DS, Barnes MJ, \& Farndale RW. Micromolar $\mathrm{Ca}^{2+}$ concentrations are essential for $\mathrm{Mg}^{2+}$-dependent binding of collagen by the integrin $\alpha_{2} \beta_{1}$ in human platelets. J Biol Chem 275, 24560-4 (2000).

26 Munnix IC, Kuijpers MJ, Auger J, Thomassen CM, Panizzi P, van Zandvoort MA, Rosing J, Bock PE, Watson SP, \& Heemskerk JW. Segregation of platelet aggregatory and procoagulant micro- 
domains in thrombus formation: regulation by transient integrin activation. Arterioscler Thromb Vasc Biol 27, 2484-90 (2007).

27 Savage B, Saldivar E, \& Ruggeri ZM. Initiation of platelet adhesion by arrest onto fibrinogen or translocation on von Willebrand factor. Cell 84, 289-97 (1996).

28 van Lier M, Lee F, Farndale RW, Gorter G, Verhoef S, Ohno-Iwashita Y, Akkerman JW, \& Heijnen $\mathrm{HF}$. Adhesive surface determines raft composition in platelets adhered under flow. J Thromb Haemost 3, 2514-25 (2005).

29 Raynal N, Hamaia SW, Siljander PR, Maddox B, Peachey AR, Fernandez R, Foley LJ, Slatter DA, Jarvis GE, \& Farndale RW. Use of synthetic peptides to locate novel integrin $\alpha_{2} \beta_{1}$-binding motifs in human collagen III. J Biol Chem 281, 3821-31 (2006).

30 Knight CG, Morton LF, Onley DJ, Peachey AR, Messent AJ, Smethurst PA, Tuckwell DS, Farndale RW, \& Barnes MJ. Identification in collagen type I of an integrin $\alpha_{2} \beta_{1}$-binding site containing an essential GER sequence. J Biol Chem 273, 33287-94 (1998). 


\title{
Chapter 3
}

\author{
Non-redundant roles of phosphoinositide 3-kinase \\ isoforms $\alpha$ and $\beta$ in glycoprotein $\mathrm{V}$-induced platelet \\ signaling and thrombus formation
}

Gilio K, Munnix IC, Mangin P, Cosemans JM, Feijge MA, van der Meijden PE, Olieslagers $\mathrm{S}$, Chrzanowska-Wodnicka MB, Lillian R, Schoenwaelder S, Koyasu S, Sage SO, Jackson SP, and Heemskerk JW

J Biol Chem. 2009;284:33750-62

Reprinted with permission 


\begin{abstract}
Platelets are activated by adhesion to vascular collagen via the immunoglobulin receptor, glycoprotein VI (GPVI). This causes potent signaling towards activation of phospholipase $\mathrm{C}_{\gamma 2}$, which bears similarity to the signaling pathway evoked by $\mathrm{T}$ - and B-cell receptors. Phosphoinositide 3-kinase $\left(\mathrm{PI}_{3} \mathrm{~K}\right)$ plays an important role in collagen-induced platelet activation, as this activity modulates the autocrine effects of secreted ADP. Here, we identified the $\mathrm{PI}_{3} \mathrm{~K}$ isoforms directly downstream of GPVI in human and mouse platelets, and determined their role in GPVIdependent thrombus formation. The targeting of platelet $\mathrm{Pl}_{3} \mathrm{~K} \alpha$ or $\beta$ strongly and selectively suppressed GPVI-induced $\mathrm{Ca}^{2+}$ mobilization and inositol 1,4,5-triphosphate production, thus demonstrating enhancement of phospholipase $\mathrm{C}_{2}$ by $\mathrm{Pl}_{3} \mathrm{~K} \alpha / \beta$. That $\mathrm{Pl}_{3} \mathrm{~K} \alpha$ and $\beta$ have a nonredundant function in GPVI-induced platelet activation and thrombus formation was concluded from measurements of: (i) serine phosphorylation of Akt, (ii) dense granule secretion, (iii) intracellular $\mathrm{Ca}^{2+}$ rises and surface expression of phosphatidylserine under flow and, (iv) thrombus formation, under conditions where $\mathrm{Pl}_{3} \mathrm{~K} \alpha / \beta$ was blocked or $\mathrm{p} 85 \alpha$ was deficient. In contrast, GPVI-induced platelet activation was insensitive to inhibition or deficiency of $\mathrm{Pl}_{3} \mathrm{~K} \delta$ or $\gamma$. Furthermore, $\mathrm{PI}{ }_{3} \mathrm{~K} \alpha / \beta$ but not $\mathrm{Pl}_{3} \mathrm{~K} \gamma$, contributed to GPVI-induced Rap1b activation and, surprisingly, also to Rapıb-independent platelet activation via GPVI. Together, these findings demonstrate that both $\mathrm{PI}_{3} \mathrm{~K} \alpha$ and $\beta$ isoforms are required for full GPVI-dependent platelet $\mathrm{Ca}^{2+}$ signaling and thrombus formation, partly independently of Rapıb. This provides a new mechanistic explanation for the anti-thrombotic effect of $\mathrm{Pl}_{3} \mathrm{~K}$ inhibition, and makes $\mathrm{Pl}_{3} \mathrm{~K} \alpha$ an interesting new target for antiplatelet therapy.
\end{abstract}

\title{
Introduction
}

Exposed collagen in a damaged vessel wall activates platelets via their immunoglobulin family receptor, glycoprotein VI (GPVI), by using a complex signal transduction pathway, which is reminiscent to the pathway employed by immune receptors in T- and B-cells $(1,2)$. In platelets, tyrosine phosphorylation of the Fc receptor $\gamma$-chain, linked to GPVI via Src family kinases, leads to a cascade of protein phosphorylation events, cumulating in the activation of phospholipase $\mathrm{C}_{\gamma 2}$ (PLC $\gamma_{2}$ ). This key effector enzyme triggers many downstream events including: production of inositol 1,4,5-trisphosphate (InsP3); mobilization of cytosolic $\mathrm{Ca}^{2+}{ }^{2}$ activation of integrin $\alpha \mathrm{llb} \beta_{3}$; secretion of platelet granules loaded with autocrine stimulating agents (ADP, ATP); and exposure of negatively charged phosphatidylserine (PS) at the platelet surface to ensure coagulation $(1,3,4)$. All these responses are potently triggered by GPVI ligands which, besides collagen, include collagen-related peptides and the snake venom convulxin (5-7).

One of the GPVI-induced signaling events contributing to PLC $\gamma_{2}$ activation is activation of the protein/lipid kinase, phosphoinositide 3-kinase $\left(\mathrm{Pl}_{3} \mathrm{~K}\right)$ in both human and mouse platelets (8-11). Evidence for this role came from the finding that in platelets stimulated with GPVI agonists, the $\mathrm{p}_{5} \alpha$ regulatory subunit of $\mathrm{Pl}_{3} \mathrm{~K}$ co-precipitates with the $\mathrm{Fc}$ receptor $\gamma$-chain and the LAT adaptor protein (8). The p85a subunit pulls p110 catalytic subunits to the membrane, where they catalyze the formation of 3-phosphorylated inositol phospholipids, primarily the phosphoinositide 3,4,5-trisphosphate $\left[\mathrm{PI}(3,4,5) \mathrm{P}_{3}\right](10)$.

Currently, there is evidence that individual class I PI3K isoforms, which are distinguished according to their catalytic subunits, have specific cellular and physiological functions. For in- 
stance, the p110 $\alpha$ isoform $\left(\mathrm{Pl}_{3} \mathrm{~K} \alpha\right)$ has been implicated in oncogenesis, and isoform-selective $\mathrm{Pl}_{3} \mathrm{~K} \alpha$ inhibitors can reduce tumor formation (12). The p110y isoform ( $\left.\mathrm{Pl}_{3} \mathrm{~K} \gamma\right)$ is involved in innate immunity and various inflammatory diseases (13), while $p 110 \delta$ has a more important role in adaptive immunity, e.g. in T- and B-cells (14). Human and mouse platelets contain four different $\mathrm{Pl}_{3} \mathrm{~K}$ isoforms, among which the class IA catalytic subunits, $\mathrm{p}_{110} \alpha, \beta$ and $\delta\left(\mathrm{Pl}_{3} \mathrm{~K} \alpha, \beta\right.$ and $\left.\delta\right)$ and the class IB catalytic subunit, p110y ( $\left.\mathrm{PI}_{3} \mathrm{~K} \gamma\right)(15-17)$. For class IA, the corresponding regulatory subunits are $p_{5}{ }_{5} \alpha / \beta p_{55} \alpha / \gamma$ and $p_{50} \alpha$, while for class IB the regulatory subunit is p101 $\gamma$. Structural studies in other cells have indicated that the regulatory class IA subunits, particularly $p 85 \alpha$, can interact with tyrosine kinase-linked receptors via the $\mathrm{SH}_{2}$ domains (18). In contrast, class IB isoforms may rather interact with $\mathrm{G}$-protein coupled receptors (16). This concept was recently challenged by the observation that, in platelets, both $\mathrm{PI}_{3} \mathrm{~K} \beta$ and $\gamma$ are activated via the $\mathrm{P}_{2} \mathrm{Y}_{12}$ receptor for ADP, which is coupled to $\mathrm{Gi}$, and that both isoforms contribute to integrin $\alpha$ llb $\beta_{3}$ activation and platelet aggregation (17,19-21). Hence, it is clear that $\mathrm{Pl}_{3} \mathrm{~K}$ isoforms can be activated by other platelet receptors than only GPVI.

To date, it is debated which of the $\mathrm{Pl}_{3} \mathrm{~K}$ isoforms become directly activated by GPVI signaling, and which are activated indirectly, e.g. following ADP receptor stimulation. Also unclear is which are the downstream events mediated by the various isoforms. Reasons for this lack of clarity include: (i) the large contribution to GPVI-induced responses of secondary, autocrine stimulators particularly ADP and thromboxane $(1,17,22)$; (ii) the proposed stimulation by $\mathrm{Pl}_{3} \mathrm{~K}$ and its product $\mathrm{PIP}_{3}$ to $\mathrm{Ca}^{2+}$ entry rather than to PLC activity $(23,24)$; (iii) the limited knowledge on the effector targets of $\mathrm{Pl}_{3} \mathrm{~K}$ and $\mathrm{PIP}_{3}$ in platelets, of which only PDK and Akt are well studied $(21,25)$; (iv) the observation that $1110 \delta$ is not a major isoform implicated in GPVI-induced platelet activation (26); ( $v$ ) the limited availability of mice deficient in $\mathrm{Pl}_{3} \mathrm{~K}$ subunits.

One possible, poorly explored target of $\mathrm{Pl}_{3} \mathrm{~K}$ in platelets is the small GTPase Rapıb, which is highly expressed in these cells, and is considered to play a key role in the activation process particularly towards $\alpha \mathrm{llb} \beta_{3}$ activation. Platelet agonists such as ADP and thrombin produce the active GTP-bound form of Rap1b, partly in a Pl3K-dependent manner $(27,28)$. Recent findings suggest that $\mathrm{Pl}_{3} \mathrm{KK} \beta$ is the main isoform responsible for the ADP-induced activation of Rap1b and Akt, while $\mathrm{Pl}_{3} \mathrm{~K} \gamma$ contributes to $\alpha$ llb $\beta_{3}$ activation by a separate pathway (21). However, whether and how Rap1b is activated by GPVI is unresolved, as is the role of different $\mathrm{PI}_{3} \mathrm{~K}$ isoforms herein.

In the present paper, we studied the identity of the $\mathrm{Pl}_{3} \mathrm{~K}$ isoforms downstream of GPVI, and determined their role in platelet activation and thrombus formation. To discriminate between direct and indirect GPVI-induced effects, the cells were stimulated in the presence of autocrine stimulation inhibitors, blocking the signaling contributions of both ADP and thromboxane. For the studies, we combined a pharmacological approach using a panel of isoformspecific $\mathrm{Pl}_{3} \mathrm{~K}$ inhibitors with experiments using mice deficient in distinct $\mathrm{Pl}_{3} \mathrm{~K}$ catalytic or regulatory subunits. We find that $\mathrm{Pl} 3 \mathrm{~K} \alpha$ and $\beta$ as key mediators of GPVI-induced thrombus formation, and show that these isoforms affect the activity of both PLC $\gamma_{2}$ and Rap1b. 


\section{Experimental procedures}

\section{Mouse strains}

Mice deficient in the $8_{5} \alpha$ regulatory $\mathrm{Pl}_{3} \mathrm{~K}$ subunit had a $\mathrm{C}_{57} \mathrm{BL} / 6$ genetic background $(11,29)$.

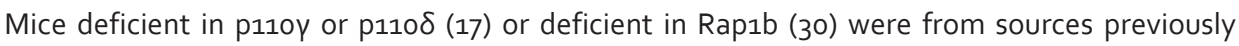
described. All knock-out mice had normal platelet counts. Wildtype mice were used of the same background and same breeding program. Animal experiments were approved by the local animal experimental committees.

\section{Materials}

Fura-2 and Fluo-3 acetoxymethyl esters, Pluronic F-127 and Oregon green 488-labeled fibrinogen (OG-fibrinogen) were from Molecular Probes; OG-annexin A5 was from Nexins Research; FITC-labeled anti-human CD62 monoclonal antibody (mAb) was from Sanquin. Antibodies against Akt were from Cell Signaling Technology; anti-Rap1 antibody was from BD Biosciences. LY294002 and H-Phe-Pro-Arg chloromethyl ketone (PPACK) were from Calbiochem. Wortmannin, apyrase (grade V), $\alpha$-thrombin and MRS-2179, a $\mathrm{P}_{2} \mathrm{Y}_{1}$ antagonist were from Sigma. Inhibitors of $\mathrm{Pl}_{3} \mathrm{~K}$ isoforms (Suppl. Table 1 ) and control substance PIK-112 were kind gifts from the Baker Heart Research Institute, and synthesized as described (17,31). Cangrelor (ARC69931MX), a P2Y12 antagonist, was kindly provided by The Medicines Company. Other reagents were from sources indicated before $(32,33)$.

\section{Blood collection and platelet isolation}

For platelet isolation, blood was collected into one-sixth volume of acid-citrate-dextrose anticoagulant ( $85 \mathrm{mM}$ sodium citrate, $78 \mathrm{mM}$ citric acid, $11 \mathrm{mM}$ D-glucose). Donors gave full informed consent according to the Helsinki declaration, and had not taken medication for 2 weeks. Platelet-rich plasma (PRP) and washed platelets were obtained by centrifugation, as described for human $(7)$ and mouse $(32,33)$ platelets. Washed human platelets were resuspended in Hepes buffer pH 7.45 (137 mM NaCl, 10 mM Hepes, $2.7 \mathrm{mM} \mathrm{KCl}, 2 \mathrm{mM} \mathrm{MgSO}$, $0.42 \mathrm{mM}$ $\mathrm{D}$-glucose, $0.2 \mathrm{U} / \mathrm{ml}$ apyrase and $0.1 \%$ bovine serum albumin, $\mathrm{pH} 7.45$ ). Mouse platelets were resuspended in a modified Hepes buffer (33). Platelets were counted with a Coulter counter. For whole-blood flow experiments, human blood was collected into $40 \mu \mathrm{M}$ PPACK (34), while mouse blood was collected into $40 \mu \mathrm{M}$ PPACK plus $5 \mathrm{U} / \mathrm{ml}$ heparin (32).

Intracellular $\mathrm{Ca}^{2+}$ measurement in platelet suspensions

Human PRP $\left(2 \times 10^{8}\right.$ platelets $\left./ \mathrm{ml}\right)$ was loaded with $2.5 \mu \mathrm{M}$ Fura-2 acetoxymethyl ester in the presence of aspirin $(100 \mu \mathrm{M})$ and apyrase $(0.2 \mathrm{U}$ ADPase $/ \mathrm{ml})$ at $37^{\circ} \mathrm{C}$ for $45 \mathrm{~min}$ (35). The loaded platelets were resuspended in Hepes buffer $\mathrm{pH} 7.45\left(1 \times 10^{8} / \mathrm{ml}\right)$, and were used within $90 \mathrm{~min}$. Before addition of agonist, the cells were preincubated with apyrase $(0.1 \mathrm{U} / \mathrm{ml})$ and ADP receptor blockers ( $40 \mu \mathrm{M}$ MRS-2179 for $\mathrm{P}_{2} \mathrm{Y}_{1}$ and $10 \mu \mathrm{M}$ ARC-69931MX for $\mathrm{P}_{2} \mathrm{Y}_{12}$ ) to inhibit autocrine stimulation. In some experiments, apyrase was added at a high concentration (0.6 U/ml). Platelet preincubation with indicated $\mathrm{Pl}_{3} \mathrm{~K}$ inhibitors or Me2SO vehicle was for $10 \mathrm{~min}\left(37^{\circ} \mathrm{C}\right)$. Calcium responses were recorded under stirring with an SLM-Aminco or a Cairn Research spectrofluorometer, at alternate excitation wavelengths of 340 and $380 \mathrm{~nm}\left(37^{\circ} \mathrm{C}\right)$. The $340 / 380-\mathrm{nm}$ ratio values were converted into nanomolar concentrations of $\left[\mathrm{Ca}^{2+}\right]_{\mathrm{i}}$ as described (35). Sepa- 
rate calibrations were done for incubations containing colored $\mathrm{Pl}_{3} \mathrm{~K}$ inhibitors. Rises in $\left[\mathrm{Ca}^{2+}\right]_{i}$ were expressed as 5 min-time integrals, to quantify prolonged $\mathrm{Ca}^{2+}$ signaling effects (36).

\section{Platelet aggregation and flow cytometry}

Human PRP was pretreated with aspirin, and platelets were washed. The washed platelets were preincubated for 10 min with autocrine stimulation inhibitors (see above) and $\mathrm{Pl}_{3} \mathrm{~K}$ blockers or $\mathrm{Me} 2 \mathrm{SO}$ vehicle. After $10 \mathrm{~min}$ of activation, samples were analyzed by flow cytometry for Pselectin expression using FITC-anti-CD62 mAb (1:100), or for $\alpha$ llb $\beta_{3}$ activation using FITClabeled PAC1 mAb (37).

Washed mouse platelets were prepared from PRP (21), and preincubated with a cocktail of autocrine stimulation inhibitors (100 $\mu \mathrm{M}$ MRS-2179, $10 \mu \mathrm{M}$ AR-C69931MX and $10 \mu \mathrm{M}$ indomethacin). Platelet aggregation was measured, as described (21). OG488-fibrinogen binding to the platelets was assessed by flow cytometry (33).

\section{InsP measurement}

Accumulation of InsP1 due to InsP3 production was measured with a IP-One Elisa kit from CisBio, determining InsP1 levels. Aspirin-treated platelets $\left(1 \times 10^{8} / \mathrm{ml}\right)$ were preincubated with ADP receptor blockers, $\mathrm{LiCl}(1 \mathrm{mM})$, and $\mathrm{Me} 2 \mathrm{SO}$ vehicle or $\mathrm{Pl}_{3} \mathrm{~K}$ blocker. After stimulation for 5-10 min with convulxin or thrombin, the platelets were lysed. Cell lysates were incubated with Ins 1 1-HRP conjugate and anti-InsP1 mAb, according to the manufacturer's instructions.

\section{Activation of Akt and Rapı by western blotting}

Akt activation was measured by Western blot analysis of platelet lysates. A polyclonal antiphosphoserine-473 Akt Ab was used to detect active Akt, and a polyclonal anti-Akt Ab to determine total Akt (19). Activation of Rap1 was measured in platelet lysates by selective precipitation of GTP-bound Rap1 using GST-RaIGDS bound to gluthatione-Sepharose (27). Western blotting was performed with anti-Rap1 Ab $(250 \mathrm{ng} / \mathrm{ml})$ and a sheep anti-mouse HRP-coupled secondary Ab (1:5000).

\section{Thrombus formation and procoagulant activity under flow}

Perfusion of PPACK-anticoagulated human blood (34) and of PPACK/heparin-anticoagulated mouse blood (32) was done in the absence of coagulation as described. Blood samples were preincubated with $\mathrm{Pl}_{3} \mathrm{~K}$ inhibitor or $\mathrm{Me} 2 \mathrm{SO}$ vehicle for $10 \mathrm{~min}$. Blood was flowed through a transparent, parallel-plate flow chamber, containing a collagen-coated coverslip, at defined shear rate for $4 \mathrm{~min}$. The thrombi in flow chambers were stained by rinse with Hepes buffer $\mathrm{pH}$ 7.45, supplemented with $1 \mathrm{U} / \mathrm{ml}$ heparin, $2 \mathrm{mM} \mathrm{CaCl} 2$ and $\mathrm{OG}_{488}$-annexin $\mathrm{A}_{5}(1 \mu \mathrm{g} / \mathrm{ml})$. Microscopic images were recorded in real-time using a Visitech imaging system, equipped with two intensified CCD cameras (34). Bright-field contrast and fluorescence digital images were taken from $>10$ randomly chosen fields. ImagePro software (Media Cybernetics) was used to quantify platelet deposition (38). 
Intracellular $\mathrm{Ca}^{2+}$ measurement under flow

Human PRP $\left(2 \times 10^{8} / \mathrm{ml}\right)$ was incubated with $7 \mu \mathrm{M}$ Fluo-3 acetoxymethyl ester for $45 \mathrm{~min}$ at $20^{\circ} \mathrm{C}$ under gentle rotation. Washed mouse platelets $\left(2 \times 10^{8} / \mathrm{ml}\right)$ were incubated with $5 \mu \mathrm{M}$ Fluo-3 acetoxymethyl ester plus $0.2 \mathrm{mg} / \mathrm{ml}$ Pluronic F-127. The dye-loaded cells were added to autologous blood (10\% labeled platelets), and used for flow experiments within $1 \mathrm{~h}$. Fluorescence images were recorded during high-speed $(5 \mathrm{~Hz}$ ) perfusion of blood over a collagen surface (39). Off-line, regions-of-interest representing 1 adhered cell were analyzed for fluorescence changes. Raw fluorescence data were converted into F/Fo values by pseudo-ratio analysis, and then into nanomolar concentrations of $\left[\mathrm{Ca}^{2+}\right]_{\mathrm{i}}(40)$. For quantification, traces from individual cells were superimposed, so that frame numbers of initial $\left[\mathrm{Ca}^{2+}\right]_{i}$ rises coincided.

\section{Statistics}

Differences between groups were tested with a Mann-Whitney $U$ test or ANOVA. Effects of inhibitors were tested with a Student's paired t test. The statistical package for social sciences was used (SPSS 15.0).

\section{Results}

Prominent roles of $\mathrm{Pl} / 3 \mathrm{~K} \alpha$ and $\mathrm{B}$ isoforms in GPVI-induced $\mathrm{Ca}^{2+}$ responses

Although $\mathrm{Pl}_{3} \mathrm{~K}$ activity has been implicated in GPVI-mediated platelet activation, it is unclear whether its role is direct or indirect, e.g. via autocrine ADP release and $\mathrm{P}_{2} \mathrm{Y}_{12}$ signaling. Another proposal is that $\mathrm{Pl}_{3} \mathrm{~K}$ may specifically stimulate GPVI-induced $\mathrm{Ca}^{2+}$ entry rather than mobilization of $\mathrm{Ca}^{2+}$ from internal stores (23). To investigate this further, human Fura-2-loaded platelets were activated with GPVI agonist under conditions where indirect effects of autocrine stimulators (thromboxane $A_{2}$ and ADP) were prevented with aspirin and a high concentration of ADP-degrading apyrase $(0.6 \mathrm{U} / \mathrm{ml})$. When platelets were stimulated with CRP in the presence of extracellular EGTA (where $\mathrm{Ca}^{2+}$ is only mobilized from internal stores), the general $\mathrm{Pl}_{3} \mathrm{~K}$ inhibitor wortmannin $(0.1 \mu \mathrm{M})$ caused $\sim 60 \%$ suppression of the $\mathrm{Ca}^{2+}$ signal (Fig. $1 \mathrm{~A}$ ). In the presence of extracellular $\mathrm{CaCl}_{2}$ (where $\mathrm{Ca}^{2+}$ entry takes place in addition), wortmannin had a similar inhibitory effect on the $\mathrm{Ca}^{2+}$ signal. The treatment of platelets with another general $\mathrm{Pl}_{3} \mathrm{~K}$ inhibitor, LY-294002 $(25 \mu \mathrm{M})$, was as effective as wortmannin. Furthermore, similar effects were obtained with both inhibitors, when platelets were stimulated with the GPVI ligand, convulxin (Fig. 1B). To directly determine the inhibitor effect on $\mathrm{Ca}^{2+}$ entry, platelets were first stimulated with GPVI ligand causing $\mathrm{Ca}^{2+}$ mobilization, after which $\mathrm{CaCl}_{2}$ was added and $\mathrm{Ca}^{2+}$ entry was allowed. Strikingly, wortmannin suppressed both parts of the $\mathrm{Ca}^{2+}$ signal at similar extent (Fig. 1C). A control experiment indicated that blocking anti-GPIb antibodies did not affect the convulxin-induced $\mathrm{Ca}^{2+}$ response (not shown), thus excluding involvement of GPIb-induced $\mathrm{Ca}^{2+}$ signal generation with this agonist.

Interestingly, treatment of the platelets with wortmannin or LY-294002 caused only small inhibition of the thrombin-evoked $\mathrm{Ca}^{2+}$ responses in both EGTA and $\mathrm{CaCl}_{2}$ medium (Fig. $1 \mathrm{~B}$ ). Together, these data suggest that $\mathrm{Pl}_{3} \mathrm{~K}$ activity primarily enhances $\mathrm{PLC} \mathrm{C}_{2}$-evoked $\mathrm{Ca}^{2+}$ mobilization from stores, whereas it only secondarily affects $\mathrm{Ca}^{2+}$ entry. 
Supplementary table 1. Inhibition of $\mathrm{Pl}_{3} \mathrm{~K}$ lipid kinase activity for purified enzymes in vitro. Listed are $\mathrm{IC}_{50}$ values for the indicated compounds, regarding inhibition of the catalytic activity of recombinant class I $\mathrm{Pl}_{3} \mathrm{~K}$ isoforms in cell-free system. Data were drawn from appropriate patent and publication information, see Refs. $(31,41)$.

\begin{tabular}{lcllll}
\hline Compound & $\begin{array}{c}\text { Relative } \\
\text { specificity }\end{array}$ & $\alpha$ & $\beta$ & $\gamma$ & $\mathrm{IC}_{50}(\mu \mathrm{M})$ \\
\cline { 2 - 5 } PIK-112 & none & $>100$ & $>100$ & $>100$ & $>100$ \\
PIK-75 & $\alpha$ & 0.008 & 0.34 & 0.9 & $>20$ \\
PI-103 & $\alpha / \beta$ & 0.004 & 0.018 & $>500$ & $>20$ \\
YM-024 & $\alpha / \delta$ & 0.30 & 2.65 & 9.1 & 0.33 \\
TGX-221 & $\beta$ & 7.0 & 0.008 & 3.5 & 0.2 \\
AS-252424 & $\gamma$ & 1.1 & $>20$ & 0.035 & $>20$ \\
IC-87114 & $\delta$ & $>100$ & 75 & 29 & 0.1 \\
LY-294002 & $\alpha-\delta$ & 0.50 & 1.0 & n.d. & 0.57 \\
Wortmannin & $\alpha-\delta$ & 0.001 & 0.003 & n.d. & 0.001 \\
\hline
\end{tabular}

Various new isoform-specific catalytic site inhibitors of class I PI3Ks have been developed and used for functional studies in other cell types $(31,41)$. The selectivity of these inhibitors for $\mathrm{Pl} 3 \mathrm{~K} \alpha-\delta$ isoforms is mostly determined in cell-free in vitro studies using isolated enzymes $\left(\mathrm{IC}_{50}\right.$ values are given in Supplementary Table I). The compound PIK-75 has a far higher affinity for $\mathrm{Pl}_{3} \mathrm{~K} \alpha\left(\mathrm{IC}_{50} 8 \mathrm{nM}\right)$ than for other isoforms. At nanomolar concentrations, the compound $\mathrm{PI}-103$ inhibits both $\mathrm{Pl}_{3} \mathrm{~K} \alpha$ and $\beta$ (PIK-112 is an inactive analog). YM-024 has a relatively high affinity for $\mathrm{Pl}_{3} \mathrm{~K} \alpha$ and $\delta$ whilst TGX-211 is most active against $\mathrm{Pl}_{3} \mathrm{KK} \beta\left(\mathrm{IC}_{50} 7 \mathrm{nM}\right)$. The compounds AS252424 and IC-87114 show highest activity towards $\mathrm{Pl}_{3} \mathrm{~K} \gamma$ and $\mathrm{PI}_{3} \mathrm{~K} \delta$ isoforms, respectively. It should be remarked that effective doses of these compounds for complete inhibition of the kinase activity in adipocytes, hepatoma cell lines and platelets are reported to be in the low micromolar range $(17,21,41)$. Hence, for adequate inhibition of $\mathrm{Pl}_{3} \mathrm{~K}$ activities in intact platelets, higher doses are needed than apparent from the $\mathrm{IC}_{50}$ values. Reasons for this are the high platelet counts in experiments and the high ATP concentration in cells (at least 10-fold higher than of in vitro kinase assays).

For the above-mentioned inhibitors, dose-effect relations were determined for convulxininduced $\mathrm{Ca}^{2+}$ responses in $\mathrm{CaCl}_{2}$ medium. In order to prevent autocrine stimulatory effects of thromboxane and ADP, all experiments were carried out with aspirin-treated platelets and blockers of both ADP receptors were added. Time integrals of increases in $\left[\mathrm{Ca}^{2+}\right]_{i}$ served as a read-out of the extent of GPVI-induced PLC activation (36). Strikingly, those inhibitors with a high-affinity towards $\mathrm{Pl}_{3} \mathrm{~K} \alpha(\mathrm{PIK}-75, \mathrm{PI}-103$ and $\mathrm{YM}-024,0.5-1 \mu \mathrm{M})$ reduced the $\mathrm{Ca}^{2+}$ signal with about 50\%, while the control substance PIK-112 was completely inactive (Fig. 2A,B). In addition, the $\mathrm{Pl}_{3} \mathrm{~K} \beta$ selective inhibitor TGX-221 (0.1-2.5 $\left.\mu \mathrm{M}\right)$ caused a similar reduction in $\mathrm{Ca}^{2+}$ signal. Combined application of PIK-75/TGX-221 or PI-103/TGX-221 did not further suppress the response. In general, the isoform specific $\mathrm{Pl}_{3} \mathrm{~K} \alpha$ or $\beta$ inhibitors were similarly effective as the general Pl 3 K inhibitors, wortmannin and LY-294002. On the other hand, AS-252424 (0.1-1 $\mu \mathrm{M})$, inhibiting $\mathrm{Pl} 3 \mathrm{~K} \gamma$, was without any effect, while the $\mathrm{PI} 3 \mathrm{~K} \delta$ inhibitor IC-87114 only slightly reduced the $\mathrm{Ca}^{2+}$ response. 
A
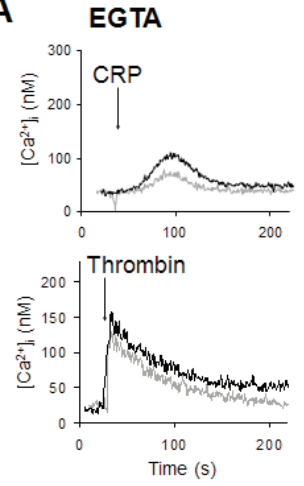

C

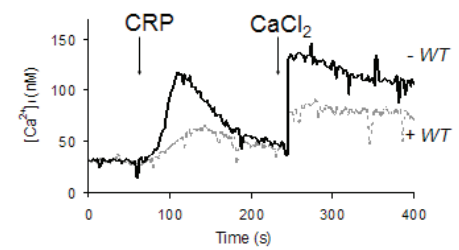

B

EGTA

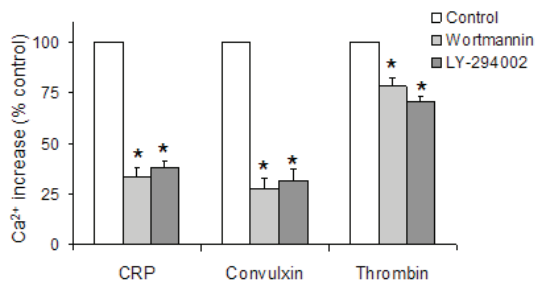

$\mathrm{CaCl}_{2}$

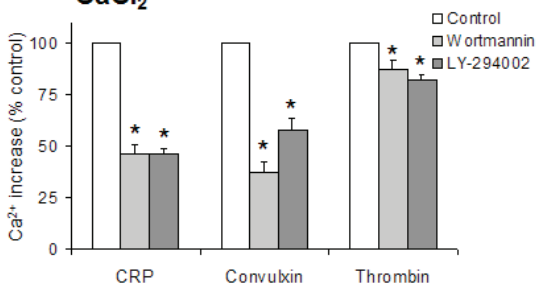

Figure 1. Major contribution of $\mathrm{Pl}_{3} \mathrm{~K}$ to $\mathrm{GPVI}$-induced versus thrombin receptor-induced $\mathrm{Ca}^{2+}$ responses in absence of autocrine mediators. Human platelets, treated with aspirin and loaded with Fura-2, were preincubated for 10 min with Me2SO vehicle (control), wortmannin $(0.1 \mu \mathrm{M})$ or LY-294002 (10 $\mu \mathrm{M})$ in the presence of apyrase $(0.6 \mathrm{U} / \mathrm{ml})$. The platelets were stimulated with GPVI ligand, CRP $(5 \mu \mathrm{g} / \mathrm{ml})$ or convulxin (70 ng/ml), or with thrombin (10 nM), in the presence of $1 \mathrm{mM} \mathrm{EGTA}$ or $1 \mathrm{mM} \mathrm{CaCl} 2$. A. Effect of wortmannin (WT) on CRP-induced $\left[\mathrm{Ca}^{2+}\right]_{\mathrm{i}}$ rises. B. Quantitative effect of wortmannin and LY-294002 on integrated $\mathrm{Ca}^{2+}$ responses in the presence of EGTA or $\mathrm{CaCl}_{2}$. Per agonist, time- $\left[\mathrm{Ca}^{2+}\right]_{i}$ integrals of the relevant control condition were set at $100 \%$. C. Effect of wortmannin on convulxin-induced $\mathrm{Ca}^{2+}$ mobilization and secondary $\mathrm{Ca}^{2+}$ entry $(1 \mathrm{mM} \mathrm{CaCl} 2)$. Representative traces are shown. Means \pm S. $E$. $(n=4-5) ;{ }^{*} p<0.05$ compared to control.

For comparison, the same panel of inhibitors was tested in measurements of thrombininduced $\mathrm{Ca}^{2+}$ responses. Here, only compounds with a high affinity towards $\mathrm{Pl}_{3} \mathrm{~K} \beta$ (i.e. $\mathrm{PI}-103$ and TGX-221) and the general PI3K inhibitors (wortmannin and LY-294002) reduced the $\mathrm{Ca}^{2+}$ signal by $15-20 \%$ (Supplementary Fig. 1). In this case, the $\mathrm{Pl}_{3} \mathrm{~K} \alpha$ inhibitor PIK-75 was without effect. Together, these results point to high and non-redundant roles of the $\mathrm{Pl}_{3} \mathrm{~K} \alpha$ and $\beta$ isoforms in $\mathrm{Ca}^{2+}$ signaling directly downstream of GPVI, but not of thrombin receptors.

Control experiments were performed to exclude that the high-affinity $\mathrm{Pl}_{3} \mathrm{~K} \alpha$ inhibitor, $\mathrm{PIK}$ 75 , acted by residual inhibition of $\mathrm{PIK}_{3} \beta$. We examined the effect of PIK-75 (0.5-1 $\left.\mu \mathrm{M}\right)$ on $\mathrm{P}_{2} \mathrm{Y}_{12}$ induced platelet responses and integrin $\alpha \mathrm{llb} \beta_{3}$ activation, since these are exclusively mediated by the $\mathrm{Pl}_{3} \mathrm{KK} \beta$ and $\gamma$ isoforms $(20,21)$. As shown in the supplementary Fig. 2, PIK-75 was completely inactive in both platelet aggregation and integrin activation in response to $A D P$, such in marked contrast to the established $\mathrm{PI}_{3} \mathrm{~K} \beta$ inhibitor, TGX-221. On the other hand, TGX-221 did not affect Akt phosphorylation at Ser473 evoked by insulin-like growth factor (IGF)-1, which is known to be mediated by only the PI3K $\alpha$ isoform (S. Kim, data not shown but see Ref. 42). 


\section{A Thrombin}
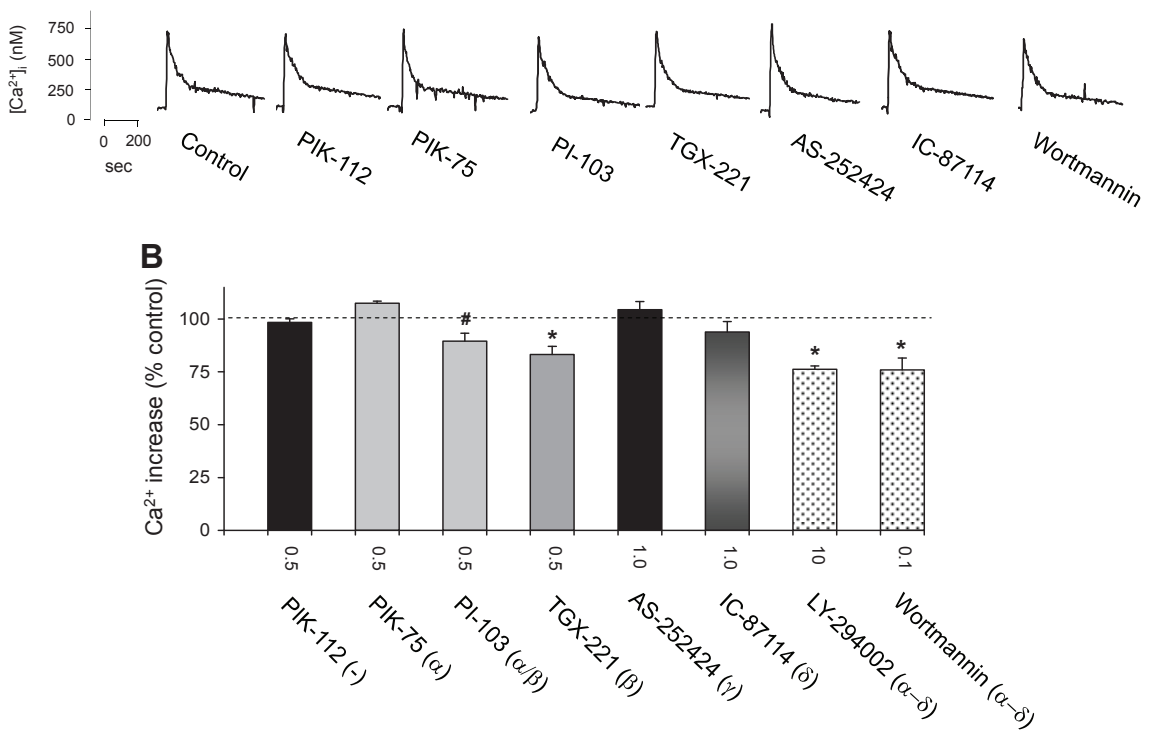

Supplementary figure 1. Inhibition of platelet $\mathrm{Pl}_{3} \mathrm{~K}$ isoforms moderately affects thrombin receptor induced $\mathrm{Ca}^{2+}$ responses. Human Fura-2-loaded platelets were treated with aspirin, preincubated with $\mathrm{ADP}$ receptor blockers, and treated with $\mathrm{Pl}_{3} \mathrm{~K}$ inhibitor, as described for Fig. 2. Shown are $\mathrm{Ca}^{2+}$ responses upon stimulation with thrombin $(10 \mathrm{nM})$. A. Representative $\left[\mathrm{Ca}^{2+}\right]_{i}$ traces; $\mathrm{B}$. inhibitor effects expressed as percentages of time- $\left[\mathrm{Ca}^{2+}\right]_{i}$ integrals relative to the control condition. Note that at a low dose of thrombin (1 nM), PIK-75, TGX-221 or the combination of both (0.5 $\mu \mathrm{M})$ suppressed the $\mathrm{Ca}^{2+}$ rise to $107 \pm 3 \%, 87 \pm 2 \%$ or $101 \pm 5 \%$ of control, respectively. Data are means \pm S.E. $(n=3-5) ;{ }^{*} p<0.05$ compared to control.

\section{Contribution of both $\mathrm{Pl} / 3 \mathrm{~K} \alpha$ and $\mathrm{B}$ isoforms to GPVI-induced signaling}

In platelets, the GPVI receptor activates the $\mathrm{PH}$-domain containing $\mathrm{PLC} \mathrm{C}_{2}$, such in contrast to thrombin receptors which activate PLC $\beta$ isoforms. For other cells, it was hypothesized that an increase in $\mathrm{PI}_{3}(3,4,5) \mathrm{P}_{3}$ level caused by $\mathrm{Pl}_{3} \mathrm{~K}$ pulls $\mathrm{PLC} \gamma_{2}$ to the membrane and increases its action (18). To verify this for GPVI-stimulated platelets (again with blocked autocrine responses), we determined overall PLC activity by measuring the accumulation of InsP1 due to $\operatorname{InsP}_{3}$ production. Platelet stimulation with convulxin led to a production of $\mathrm{InsP}_{3}$ for up to $10 \mathrm{~min}$ (Fig. $3 \mathrm{~A}$ ). Wortmannin treatment reduced the $\mathrm{InsP}_{3}$ formation and moreover confined it to $5 \mathrm{~min}$. Such a shortening of InsP3 production was not seen in thrombin-stimulated platelets. The isoform-specific inhibitors, PIK-75 or TGX-221 (targeting at $\mathrm{Pl}_{3} \mathrm{~K} \alpha$ and $\beta$, respectively) suppressed the InsP3 production to a similar extent as wortmannin, while the combination of the two did not have an additional effect (Fig. ${ }_{3} \mathrm{~B}$ ). In contrast, inhibition of $\mathrm{Pl}_{3} \mathrm{~K} \gamma$ (AS-252424) or $\mathrm{Pl}_{3} \mathrm{~K} \delta$ (IC87114) was not effective on InsP3 amounts. Hence, these results point to a GPVI-induced prolongation of $\mathrm{PLC}$ activity by $\mathrm{Pl}_{3} \mathrm{~K} \alpha / \beta$. 

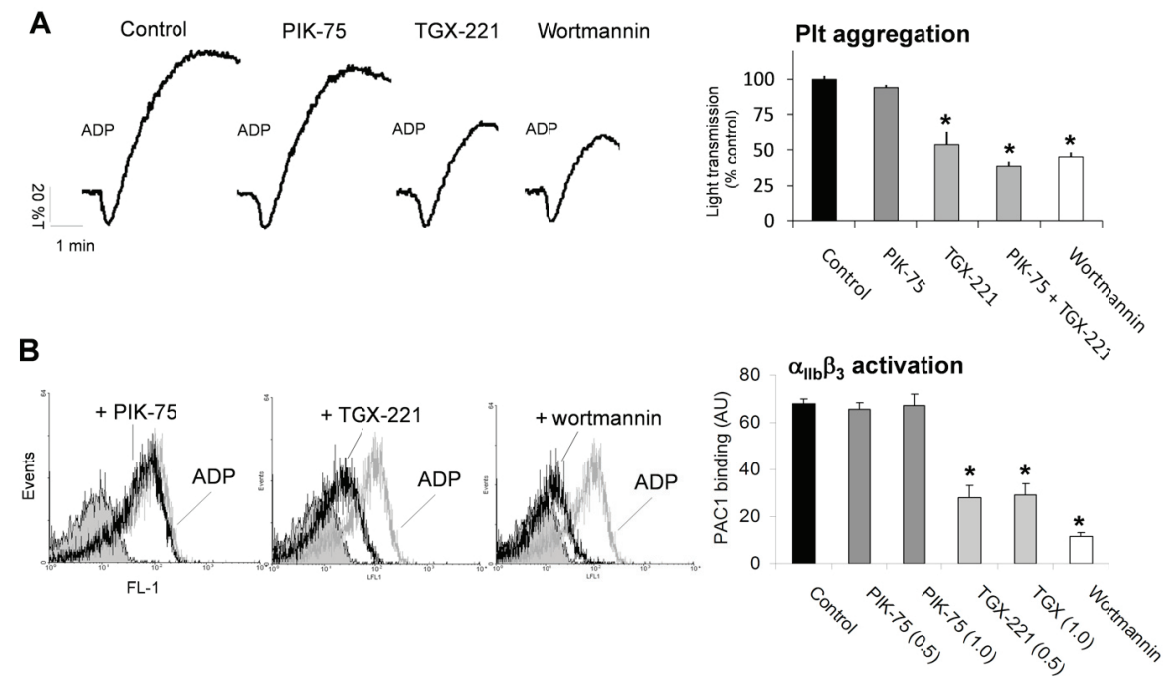

Supplementary figure 2. Specificity of $\mathrm{Pl}_{3} \mathrm{~K} \beta$ inhibitor TGX-221 for affecting ADP-induced platelet activation. Human PRP was preincubated with Me2SO vehicle (control), TGX-221 (0.5-1 $\mu \mathrm{M})$, PIK-75 $\left(0.5^{-1} \mu \mathrm{M}\right)$ or wortmannin $(1 \mu \mathrm{M})$, prior to stimulation with ADP. A. Representative light transmission aggregation traces induced by $5 \mu \mathrm{M} A D P$, and quantitative effect of inhibitors on ADP-induced platelet aggregation. B. Binding of FITC-PAC 1 to platelets stimulated with $20 \mu \mathrm{M}$ ADP (10 min). Fluorescence histograms are shown of unstimulated platelets (filled grey), vehicle controls (grey lines) and samples containing indicated inhibitor (black overlays). Means \pm S.E. $(n=4) ; * p<0.05$ compared to control.

We measured agonist-induced $\mathrm{Ser}^{473}$ phosphorylation of Akt as an established downstream effector of lipid $\mathrm{Pl}_{3} \mathrm{~K}$ (21). In platelets stimulated with convulxin (Fig. ${ }_{3} \mathrm{C}$ ) or CRP (not shown), wortmannin treatment suppressed the phosphorylation of Akt, as expected. Interestingly, also inhibition of $\mathrm{Pl}_{3} \mathrm{~K} \alpha$ (PIK-75) or $\beta$ (TGX-221) reduced this phosphorylation, while the combination was not more effective (Fig. 3D).

A key downstream effect of $\left[\mathrm{Ca}^{2+}\right]_{i}$ elevation in platelets is the secretion of $\alpha$-granules, which is measured as expression of granular P-selectin at the platelet surface. Platelet treatment with PIK-75 or TGX-221, alone or in combination, suppressed convulxin- or CRP-induced P-selectin expression with 40-50\%, i.e. to the same extent as did wortmannin or LY-294002 (Fig. 4). This points to a non-additive effect of both isoform-selective compounds on GPVIinduced secretion. 


\section{A Convulxin}
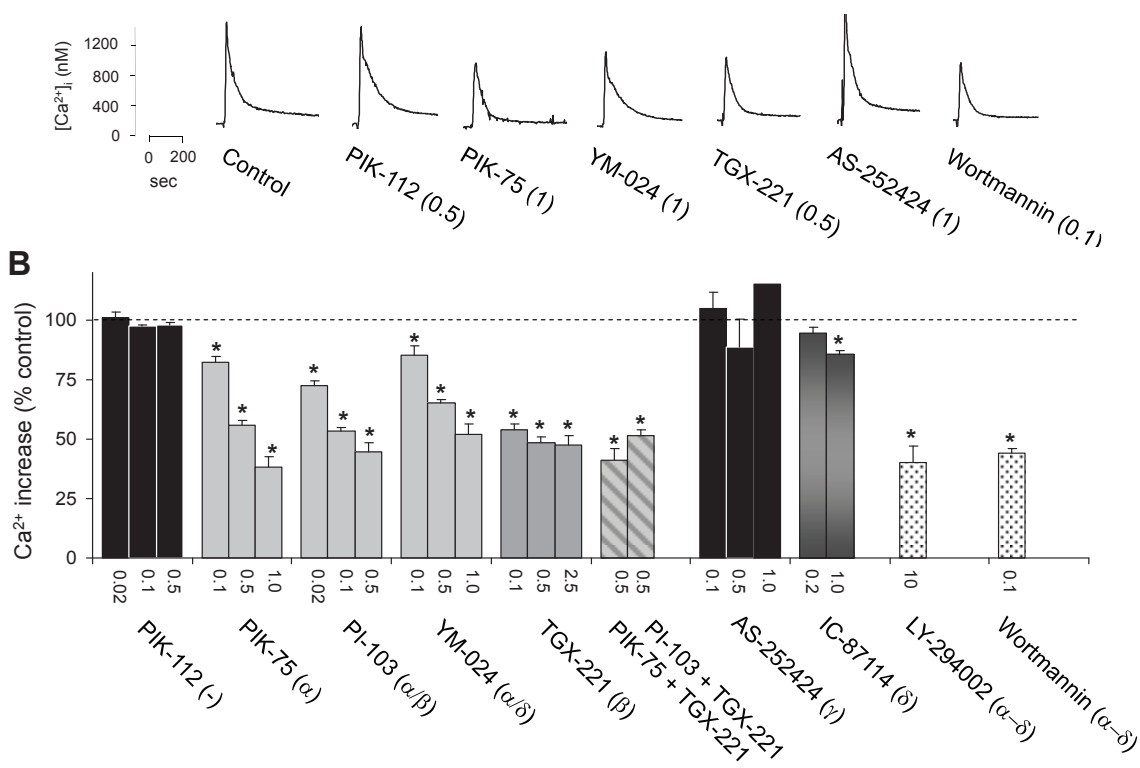

Figure 2. Inhibition of platelet $\mathrm{Pl}_{3} \mathrm{~K} \alpha$ and $\beta$ isoforms strongly suppresses $\mathrm{GPVI}$-induced $\mathrm{Ca}^{2+}$ responses. Aspirin-treated, Fura-2-loaded platelets were preincubated with ADP receptor blockers (10 $\mu \mathrm{M}$ AR-C69931MX, $40 \mu \mathrm{M}$ MRS-2179, $0.1 \mathrm{U} / \mathrm{ml}$ apyrase) and $1 \mathrm{mM} \mathrm{CaCl}$. Platelets were then treated with one of the following substances ( $\mathrm{Pl} 3 \mathrm{~K}$ isoform specificity in brackets): Me2SO vehicle (control), PIK-112 (inactive analog of PIK-75), PIK-75 ( $\alpha)$, PI-103 $(\alpha / \beta)$, YM-024 $(\alpha / \delta)$, TGX-221 $(\beta)$, AS-252424 ( $\gamma)$, IC-87114 $(\delta)$, LY-294002 $(\alpha-\delta)$ or wortmannin $(\alpha-\delta)$. Numbers give final concentrations in $\mu$ M. A. Representative $\left[\mathrm{Ca}^{2+}\right]_{i}$ traces upon stimulation with convulxin $(70 \mathrm{ng} / \mathrm{ml})$. B. Effects of inhibitors on $\mathrm{Ca}^{2+} \mathrm{re}$ sponses, expressed as percentages of time- $\left[\mathrm{Ca}^{2+}\right]_{i}$ integrals relative to the control condition. Note that at a low dose of convulxin $(7 \mathrm{ng} / \mathrm{ml})$, PIK-75, TGX-221 or the combination of both ( $0.5 \mu \mathrm{M})$ suppressed the $\mathrm{Ca}^{2+}$ rise to $51 \pm 3 \%, 40 \pm 6 \%$ or $31 \pm 1 \%$ of control, respectively. Data are means $\pm S$.E. $(n=3-5) ;{ }^{*} p<0.05$ compared to control.

Roles of $\mathrm{Pl} 3 \mathrm{~K} \alpha$ and $\mathrm{B}$ isoforms in GPVI-induced platelet activation and thrombus formation on collagen under flow

The pharmacological evidence so far points to a common role of the $\mathrm{Pl}_{3} \mathrm{~K} \alpha / \beta$ isoforms in GPVIinduced $\mathrm{Ca}^{2+}$ signaling and downstream platelet activation events. We then performed studies to determine the importance of these isoforms in flow-dependent thrombus formation at physiological shear rates. Earlier work has shown that, during platelet interaction with collagen under flow, GPVI-induced rises in $\left[\mathrm{Ca}^{2+}\right]_{i}$ are important for granule secretion, activation of integrins and exposure of PS $(22,34,42,43)$. In whole blood, supplemented with Fluo-3-loaded platelets, $\left[\mathrm{Ca}^{2+}\right]_{\mathrm{i}}$ rises were measured of individual platelets upon adhesion to collagen under flow. Interestingly, pretreatment with either PIK-75, TGX-221 or wortmannin led to a marked suppression in single cell $\mathrm{Ca}^{2+}$ responses, so that the adhered platelets showed low-amplitude spiking changes in $\left[\mathrm{Ca}^{2+}\right]_{\mathrm{i}}$ (Fig. 5). Control experiments indicated that the $\mathrm{Ca}^{2+}$ responses under flow were not influenced by aspirin and blockage of ADP receptors (not shown), thus confirming that autocrine ADP release did not contribute to this initial platelet response. Other controls indicated that these $\left[\mathrm{Ca}^{2+}\right]_{\mathrm{i}}$ rises were completely suppressed by blocking GPVI (22). 


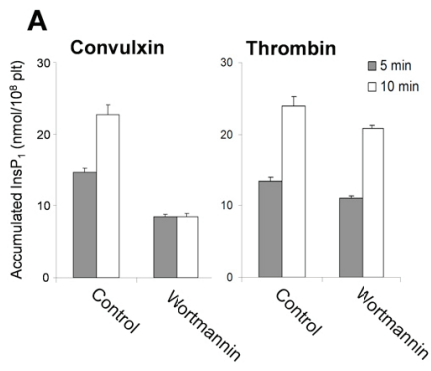

C

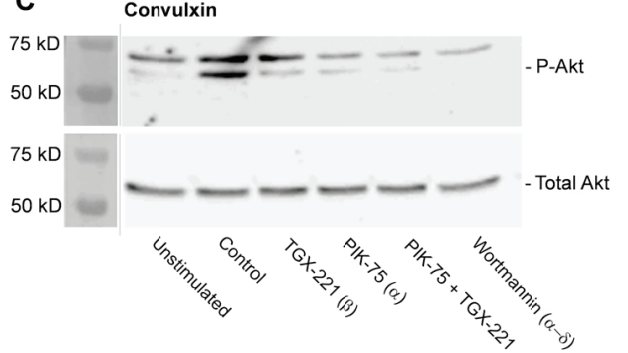

B

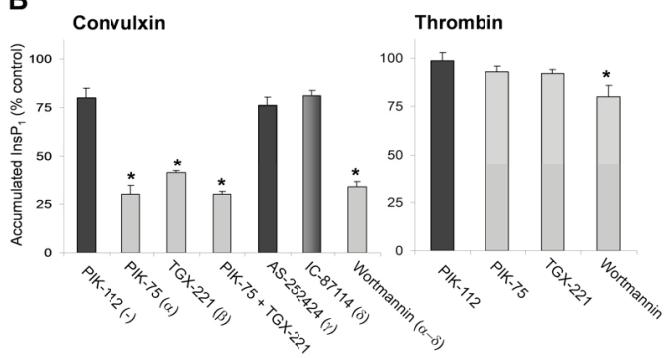

D

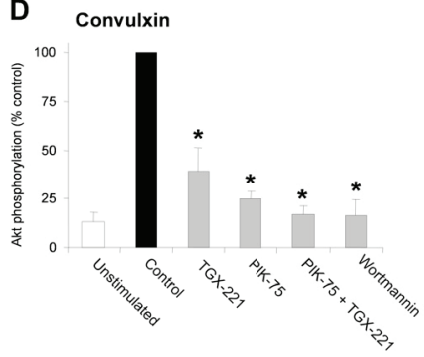

Figure 3. Contribution of $\mathrm{Pl}_{3} \mathrm{~K} \alpha$ and $\beta$ isoforms to GPVI-induced InsP3 production and Akt phosphorylation. Aspirin-treated human platelets $\left(1 \times 10^{8} / \mathrm{ml}\right)$ were preincubated with ADP receptor blockers (Fig. 2) and Me2SO vehicle (control) or $1 \mu \mathrm{M} \mathrm{Pl} 3 \mathrm{~K}$ inhibitor (25 $\mu \mathrm{M} \mathrm{LY}$-294002). A, B. Effect of $\mathrm{Pl}_{3} \mathrm{~K}$ blockers on accumulation of InsP1 due to Ins $\mathrm{P}_{3}$ production. Platelets were stimulated for 10 min with convulxin $(70 \mathrm{ng} / \mathrm{ml})$ or thrombin $(10 \mathrm{nM})$ in the presence of $1 \mathrm{mM} \mathrm{LiCl}$. C,D. Effect of $\mathrm{Pl}_{3} \mathrm{~K}$ blockers on convulxin-induced Ser ${ }^{473}$ Akt phosphorylation. C. Representative Western blots of phospho-Akt and total Akt (6o kD). D. Data are densitometric intensities of phospho-Akt/Akt, expressed as percentage of vehicle. Means \pm S.E. $(n=3-5)$; ${ }^{2}<0.05$ compared to PIK-112 or control.

The effects of inhibitors on thrombus formation were assessed from measurements of the deposition of platelets on the collagen surface (Fig. 6A). In addition, the exposure of PS of collagen-adhered platelets (detected with OG-annexin $A_{5}$ ) was measured, which response is shown by a subpopulation of the platelets in direct contact with collagen due to GPVI activation (38). Both parameters were substantially diminished with wortmannin, with the PI3K $\alpha$ inhibitors PIK75 and $\mathrm{YM}-024$, or with the $\mathrm{Pl}_{3} \mathrm{~K} \beta$ inhibitor TGX-221. In the presence of all inhibitors, platelet deposition on collagen was substantially reduced in comparison to the vehicle control (Fig. 6B). In addition, the number of PS-exposing platelets was reduced to $\sim 50 \%$ by PIK-75, YM-024 or TGX-221. On the other hand, inhibition of $\mathrm{Pl}_{3} \mathrm{~K} Y$ (AS-252424) or $\delta$ (IC-87114) was without effect. Together, these results suggest that inhibition of only $\mathrm{Pl}_{3} \mathrm{~K} \alpha$ or $\beta$ isoforms suppresses flowdependent thrombus formation on collagen, by reducing GPVI-dependent $\mathrm{Ca}^{2+}$ responses and downstream activation processes leading to platelet aggregation and PS exposure. 

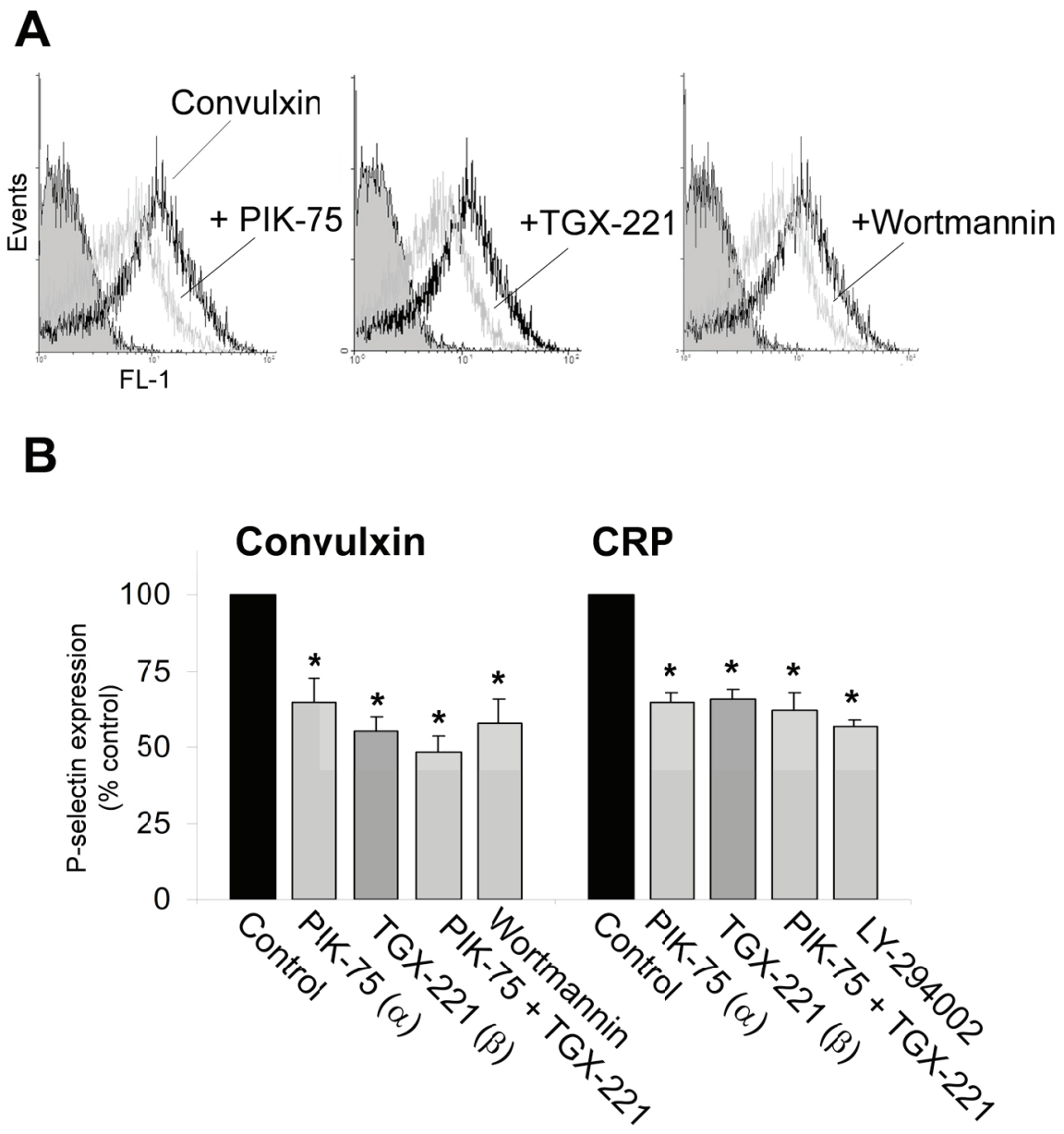

Figure 4. Contribution of $\mathrm{Pl}_{3} \mathrm{~K} \alpha$ and $\beta$ isoforms to GPVI-induced $\alpha$-granule secretion. Human washed aspirin-treated platelets $\left(1 \times 10^{8} / \mathrm{ml}\right)$ were preincubated with ADP receptor blockers (Fig. 2), and treated with Me2SO vehicle (control) or specific Pl3K inhibitor (in $\mu \mathrm{M}$ ): PIK-75 (1.0), TGX-221 (0.5), wortmannin (0.1), LY-294002 (25). The platelets were activated for $10 \mathrm{~min}$ with convulxin (70 $\mathrm{ng} / \mathrm{ml}$ ) or $\mathrm{CRP}(10 \mu \mathrm{g} / \mathrm{ml})$. A. Histogram showing effect of $\mathrm{Pl}_{3} \mathrm{~K} \alpha / \beta$ blockers on P-selectin expression (FL1 fluorescence). B. Quantitative effect of $\mathrm{Pl}_{3} \mathrm{~K}$ blockage on $\mathrm{P}$-selectin expression. Data are relative to the control condition. Means \pm S.E. $(n=3) ; * p<0.05$ compared to control.

\section{Roles of murine $\mathrm{Pl} / 3 \mathrm{~K}$ isoforms in GPVI-induced platelet activation and thrombus formation}

Platelets from mice lacking the $\mathrm{p}_{5} \alpha$ regulatory $\mathrm{Pl}_{3} \mathrm{~K}$ subunit are impaired in collagen-induced aggregation, which points to diminished GPVI signaling (11). In the $p 85 \alpha^{-1-}$ platelets, expression of the $p_{110} \alpha$ subunit $\left(\mathrm{Pl}_{3} \mathrm{~K} \alpha\right)$ is almost undetectable, while both $p_{8} \beta$ and $p_{11} \beta$ are still present at low levels $(11,29,44)$. We used blood from $p 85 \alpha^{-1-}$ mice to measure collagen-dependent $\left[\mathrm{Ca}^{2+}\right]_{\mathrm{i}}$ rises in adhered platelets, thrombus formation and PS exposure under flow. In contrast to the potent and prolonged $\mathrm{Ca}^{2+}$ responses of $\mathrm{p}_{5} \alpha^{+/+}$platelets, $\mathrm{p} 85 \alpha$-deficient platelets showed a markedly lower $\mathrm{Ca}^{2+}$ signal, which in $36 \%$ of the cells were spiking in appearance (Fig. $7 \mathrm{~A}$ ). 
Treatment of $\mathrm{p} 85 \alpha^{+/+}$blood with TGX-221 reduced the average $\mathrm{Ca}^{2+}$ signal to the level seen in p85 $\alpha^{-1-}$ platelets. Treatment of wildtype blood with YM-024 had a similar suppressive effect on the $\mathrm{Ca}^{2+}$ response $(\mathrm{p}=0.012)$. As a comparison, blood was used from $\mathrm{p} 110 \gamma^{-1-}$ mice, in which case $\mathrm{Ca}^{2+}$ responses under flow were not different from those of corresponding wildtype platelets (I. Munnix, unpublished data 2008).

\section{A}

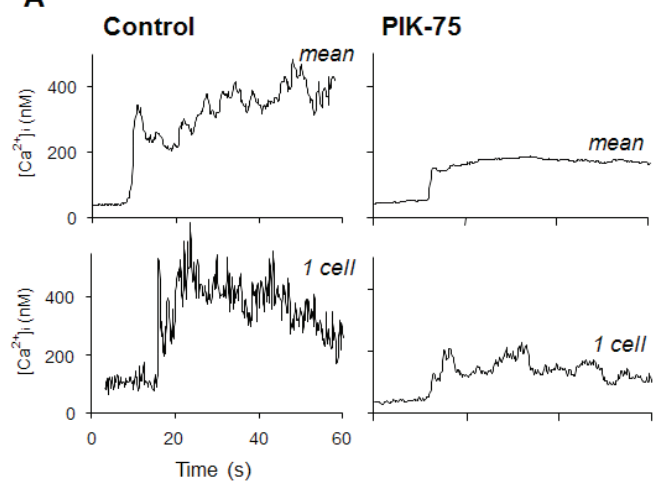

TGX-221
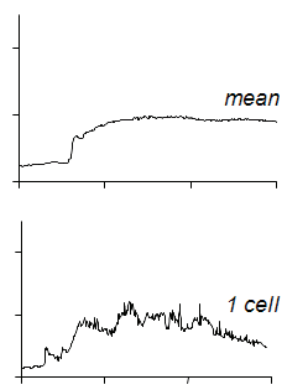

$\square \mathrm{t}=20 \mathrm{~s}$

$\square \mathrm{t}=60 \mathrm{~s}$

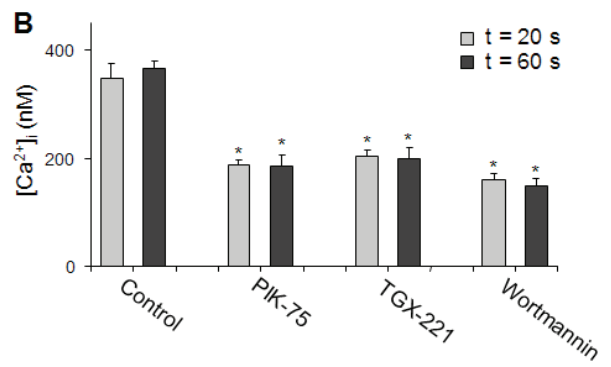

Figure 5. Role of $\mathrm{Pl}_{3} \mathrm{~K}$ in collagen-induced $\mathrm{Ca}^{2+}$ responses under flow in the absence of secondary mediators. PPACK-anticoagulated blood, containing $10 \%$ autologous Fluo-3-loaded platelets, was preincubated with Me2SO vehicle (control), PIK-75, TGX-221 or wortmannin (each $1 \mu \mathrm{M}$ ). A. Single cell $\mathrm{Ca}^{2+}$ responses during flow of blood over collagen in the presence of indicated inhibitor at a shear rate of $1000 \mathrm{~s}^{-1}$. Upper panels: mean overlays of $\left[\mathrm{Ca}^{2+}\right]_{i}$ traces from $>35$ individual platelets; lower panels: traces from representative single platelets. B. Quantification of rises in $\left[\mathrm{Ca}^{2+}\right]_{i}$ at $20 \mathrm{~s}$ (gray) or $60 \mathrm{~s}$ (dark gray) after initial platelet activation. Means \pm S.E. $(n=27-50) ; * p<0.05$ compared to control.

Measurements of flow-dependent thrombus formation on collagen showed a reduced deposition of $\mathrm{p} 85 \alpha^{-/-}$platelets, as well as a reduced PS exposure of the cells in comparison to wildtype blood (Fig. 7B). Similarly, addition of TGX-221 to $p 85 \alpha^{+/+}$blood, but not to $p 85 \alpha^{-/-}$ blood, significantly decreased thrombus formation and number of PS-exposing platelets. A role for $\mathrm{PI}_{3} \mathrm{~K} \alpha$ and $\beta$ in murine thrombus formation was further supported by inhibitor studies using blood from wildtype mice. Treatment with PIK-75 and/or TGX-221 led to a similar reduction in platelet deposition and PS exposure as treatment with the general $\mathrm{Pl}_{3} \mathrm{~K}$ inhibitor, wortmannin (Fig. $7 \mathrm{C}$ ). Together, these results demonstrated that, in mouse, the regulatory p $85 \alpha$ subunit and the catalytic $\mathrm{Pl}_{3} \mathrm{~K} \alpha$ and $\beta$ subunits are essential in flow-dependent $\mathrm{Ca}^{2+}$ signaling, $\mathrm{PS}$ exposure and thrombus formation on collagen. 
A Human

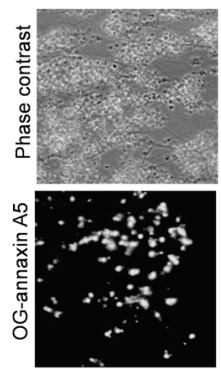

Control

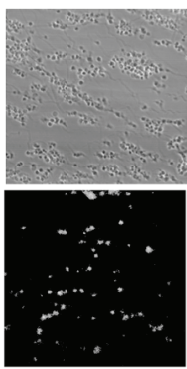

PIK-75 $(\alpha)$
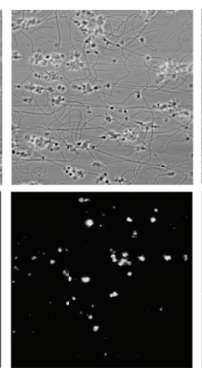

YM-024 ( $\alpha / \delta)$
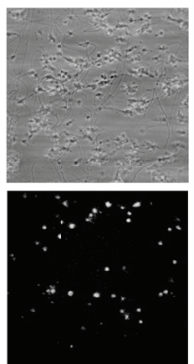

TGX-221 ( $\beta)$
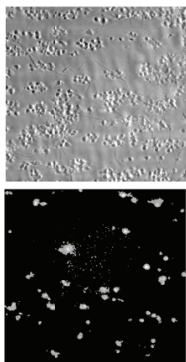

Wortmannin $(\alpha-\delta)$

B

30 PIt deposition

PS exposure
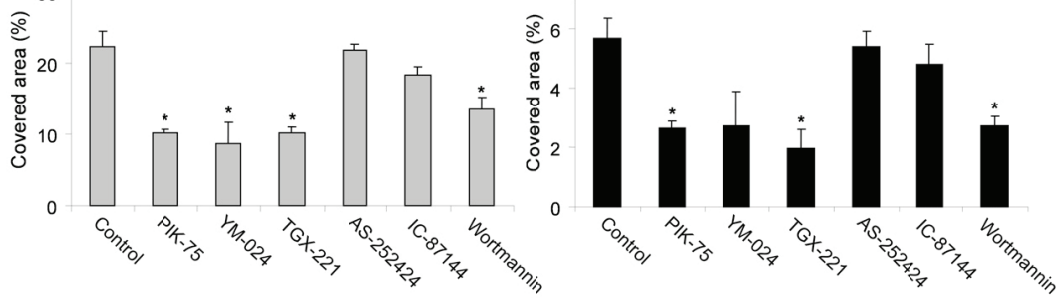

Figure 6. Role of $\mathrm{Pl}_{3} \mathrm{~K} \alpha$ and $\beta$ isoforms in collagen-induced thrombus formation and PS exposure under flow. PPACK-anticoagulated blood was flowed during 4 min over collagen at a shear rate of 1000 $\mathrm{s}^{-1}$. Preincubation was with Me2SO vehicle (control) or one of the PI3K inhibitors: PIK-75, YM-024, TGX221, AS-252424, IC-87144 or wortmannin (all $1 \mu \mathrm{M}$ ). A. Representative images of phase contrast $(120 \times 120 \mu \mathrm{m})$ and $O \mathrm{G}$-annexin A5 fluorescence $(150 \times 150 \mu \mathrm{m})$ after $4 \mathrm{~min}$ flow. B,C. Surface area coverage of all deposited platelets and PS-exposing platelets. Data are expressed as percentages of control condition. Means \pm S.E. $(n=4) ; * p<0.05$ compared to control.

Experiments carried out with washed mouse platelets, in the presence of autocrine stimulation inhibitors, confirmed that GPVI-induced activation relied on $\mathrm{Pl}_{3} \mathrm{~K} \alpha / \beta$ activity, since LY294002, PIK-75 and TGX-221 all inhibited CRP-induced aggregation (not shown). In addition, platelets from mice lacking the catalytic p110y or $1110 \delta$ subunits were essentially unaltered in aggregation, when compared to wildtype platelets (P. Mangin, unpublished data 2008). Together, this points to major functions of murine $\mathrm{p}_{110} \alpha$ and $\mathrm{p} 110 \beta \mathrm{Pl}_{3} \mathrm{~K}$ isoforms and to minor roles for $\mathrm{p} 110 \mathrm{y}$ and $\mathrm{p} 110 \delta$ in the aggregation response. 


\section{A}
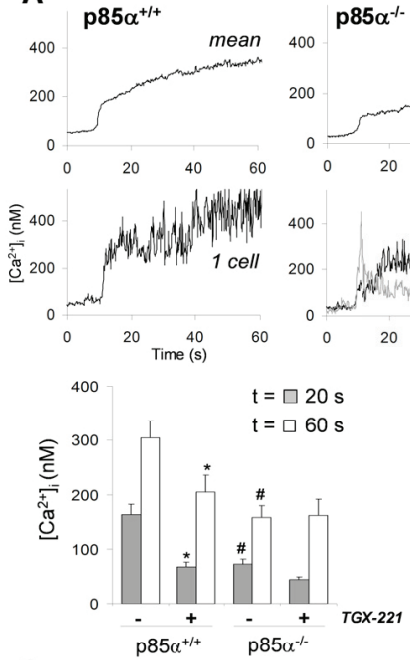

C Wildtype mouse

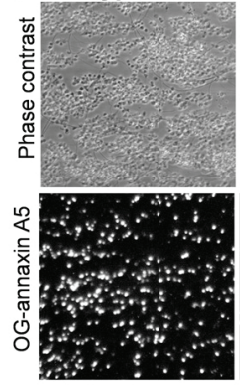

Control
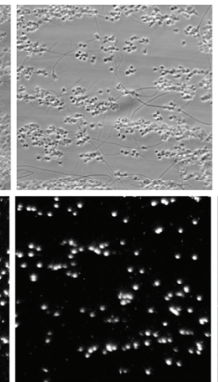

PIK-75 $(\alpha)$
B

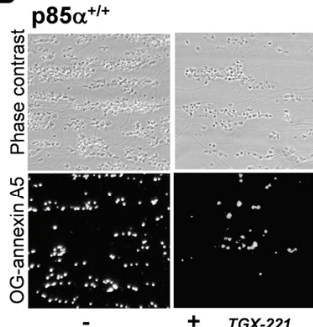

Plt deposition
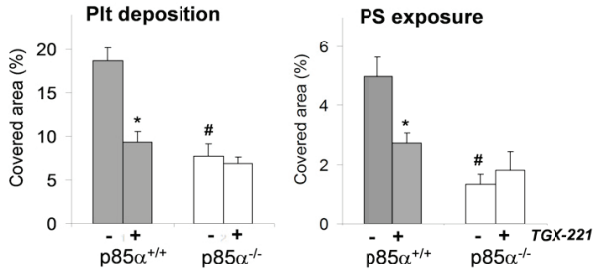

p85 $\alpha^{-1-}$

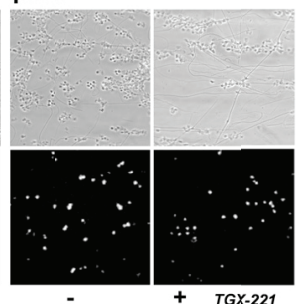

p85

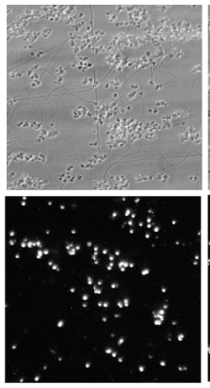

TGX-221( $\beta$ )

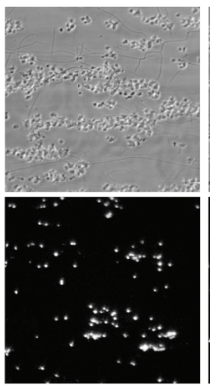

PIK-75 + TGX-221

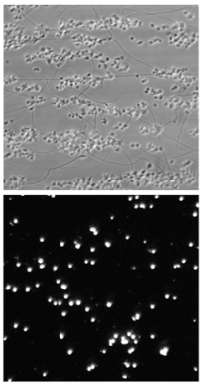

Wortmannin

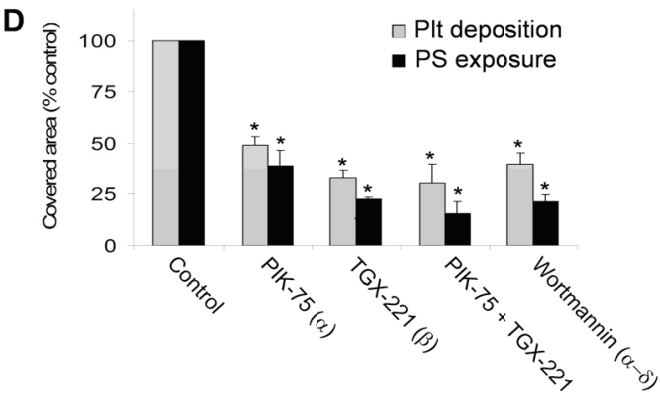

Figure 7. Murine $p_{5} \alpha$ regulates collagen-induced $\mathrm{Ca}^{2+}$ responses and thrombus formation under flow. PPACK/heparin-anticoagulated blood from $\mathrm{p} 8 \mathrm{\alpha}^{+/+}$or $\mathrm{p} 85 \mathrm{\beta}^{-/}$mice, spiked with $10 \%$ Fluo-3-loaded platelets from the same mouse strain, was flowed over collagen at $1000 \mathrm{~s}^{-1}$. The blood was preincubated with $\mathrm{Me} 2 \mathrm{SO}$ vehicle or TGX-221 $(1 \mu \mathrm{M})$. A. Contribution of $\mathrm{p} 85 \alpha$ to $\mathrm{Ca}^{2+}$ responses under high-shear perfusion. Upper panels: averaged overlays of $\left[\mathrm{Ca}^{2+}\right]_{i}$ traces from $>25$ platelets; lower panels: traces from representative single platelets. Bars show quantification of $\left[\mathrm{Ca}^{2+}\right]_{\mathrm{i}}$ rises at $20 \mathrm{~s}$ (gray) or $60 \mathrm{~s}$ (white) after initial platelet activation. B. Contribution of p85 $\alpha$ to thrombus formation and PS exposure. Shown are representative images of phase-contrast $(120 \times 120 \mu \mathrm{m})$ and OG-annexin A5 fluorescence $(150 \times 150 \mu \mathrm{m})$ of $\mathrm{p} 85 \alpha^{+/+}$and p85 $\alpha^{-/-}$thrombi. Bars represent surface area coverage of all deposited platelets and PS-exposing platelets. C. Effect of pharmacological $\mathrm{Pl}_{3} \mathrm{~K}$ inhibition on thrombus formation and PS exposure. Wildtype blood was preincubated with Me2SO vehicle (control), PIK-75, TGX-221 or wortmannin (each $1 \mu \mathrm{M})$. Data are means $\pm \mathrm{S}$.E. $(n=3-5) ; * p<0.05$ compared to vehicle control, $\# p<0.05$ compared to wildtype. 
Roles of PI3K isoforms in GPVI-induced Rap1b activation in human and mouse platelets

In platelets, the low-molecular weight GTPase, Rapıb, is a well-characterized effector downstream of $\mathrm{Pl}_{3} \mathrm{~K}$ (27). However, there is no conclusive evidence how Rap1b functions directly downstream of GPVI. Platelets from mice deficient in Rapıb were used to investigate this. In the presence of autocrine stimulation inhibitors, Rap $1 b^{-1-}$ platelets showed a $36 \%$ reduction in CRP-induced aggregation in comparison to Rap $\mathrm{b}^{+/+}$wildtypes (Fig. 8A). This suggested a direct role for this GTPase in GPVI-induced platelet aggregation. Inhibition of $\mathrm{Pl}_{3} \mathrm{~K}$ with LY-294002 markedly reduced the aggregation of Rap $1 b^{+/+}$platelets and, surprisingly, of Rap $1 b^{-/-}$platelets, with reductions of $89 \%$ and $86 \%$, respectively, in comparison to vehicle-treated controls. The observation that LY-294002 further reduced the aggregation of Rap1b ${ }^{-1-}$ platelets indicated that, even though Rapıb is involved in GPVI-mediated activation, it is not the only effector of $\mathrm{Pl}_{3} \mathrm{~K}$ in this response.

A Rap1b

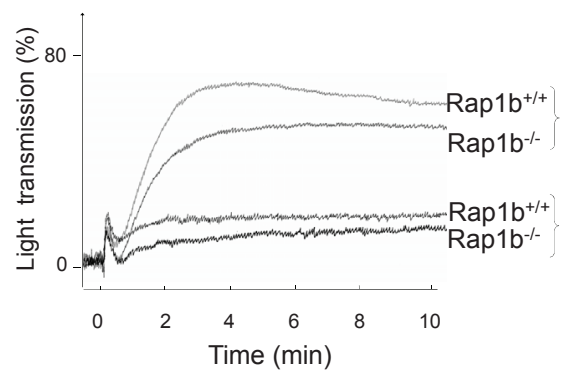

B

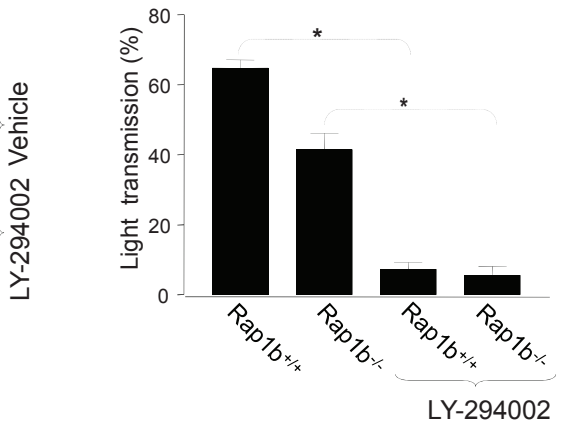

Figure 8. Contribution of Rap1b to GPVI-induced integrin $\alpha$ llb $\beta_{3}$ activation and platelet aggregation. (A) Washed platelets $\left(3 \times 10^{8} / \mathrm{ml}\right)$ from Rap $1 b^{+/+}$or Rap $1 b^{-1-}$ mice were preincubated with autocrine stimulation inhibitors (AR-C69931MX, MRS-2179 and $10 \mu \mathrm{M}$ indomethacin), in the presence of Me2SO vehicle or LY-294002 (25 $\mathrm{MM})$. Platelets were stimulated with $10 \mu \mathrm{g} / \mathrm{ml}$ CRP in the presence of purified fibrinogen $(0.5 \mathrm{mg} / \mathrm{ml})$ and aggregation was monitored. Representative aggregation traces are shown. Bars give percentages of light transmission at 3 min after CRP addition. Data are means $\pm S$.E. $(n=3-4)$; * $p<0.05$ compared to control.

To elucidate the $\mathrm{Pl}_{3} \mathrm{~K}$ isoforms implicated in Rapıb activation directly downstream of GPVI, the effect of CRP was evaluated on Rap1b-GTP levels in platelets treated with autocrine stimulation inhibitors. An inhibitor of $\alpha$ llb $\beta_{3}$ of human ( $40 \mu \mathrm{g} / \mathrm{ml}$ Reopro) or mouse (100 $\mu \mathrm{M}$ GPI-562) platelets served to suppress platelet aggregation, which process interferes with the assay. In both human and mouse platelets, CRP induced a potent activation of Rap1b (Fig. 9A,B). Treatment with LY-294002 strongly suppressed Rap1b activation with Rap1-GTP levels being reduced in human and mouse platelets by $72 \%$ and $69 \%$, respectively. Markedly, treatment with TGX-221 or PIK-75 resulted in a similar degree of reduction in Rap1b activation, both in mouse or human platelets. In contrast, effects of AS-252424 treatment were negligible. These findings demonstrate a major role for $\mathrm{Pl}_{3} \mathrm{~K} \alpha$ and $\beta$ but not $\gamma$ in Rapib activation downstream of GPVI. 


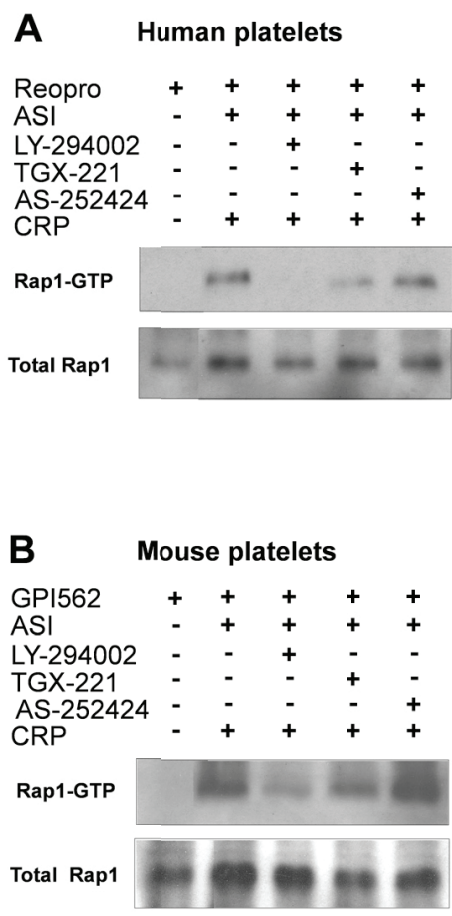

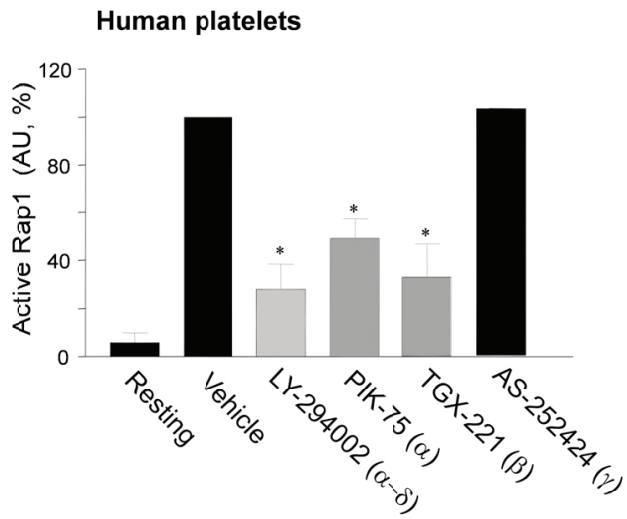

Mouse platelets

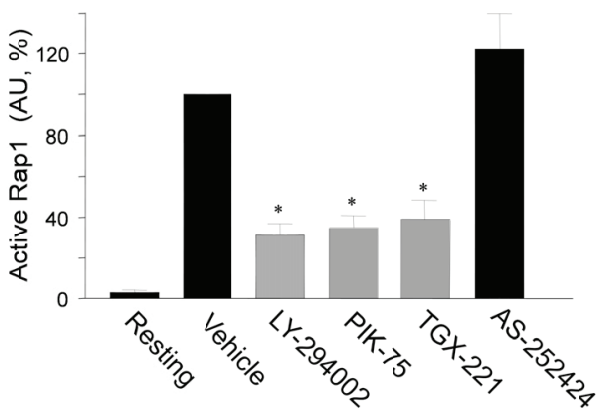

Figure 9. Contribution of $\mathrm{Pl}_{3} \mathrm{~K}$ isoforms to GPVI-induced activation of Rap1b. Washed human (A) and wildtype mouse $(B)$ platelets $\left(3 \times 10^{8} / \mathrm{ml}\right)$ were preincubated with autocrine stimulation inhibitors (ASI) (Fig. 8) and $\alpha$ llb 33 inhibitor (human: $40 \mu \mathrm{g} / \mathrm{ml}$ Reopro; mouse: $100 \mu \mathrm{M} \mathrm{GPI-562).} \mathrm{Incubations}$ contained Me2SO vehicle or Pl3K inhibitor LY-294402 (25 $\mu \mathrm{M}), \mathrm{TGX}-221(0.5 \mu \mathrm{M})$ or AS-252424 (10 $\mu \mathrm{M})$. Platelets were stimulated with $20 \mu \mathrm{g} / \mathrm{ml} \mathrm{CRP}$, lysed, and used for immunoprecipitation of GTP. Rap1. A,B. Representative Western blots of active Rap1-GTP and total Rap1. Right panels: densitometric analysis of the amount of active Rap1-GTP (arbitrary units). Means \pm S.E. $(n=3-5) ;{ }^{*} p<0.05$ compared to control.

\section{Discussion}

The present paper provides first data showing the contribution of multiple $\mathrm{Pl}_{3} \mathrm{~K}$ isoforms in GPVI-induced activation of (human) platelets, and the role of these isoforms in GPVI-dependent thrombus formation. Our findings significantly extend the earlier evidence that GPVI agonists

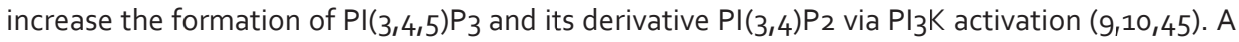
marked finding is that both $\mathrm{Pl}_{3} \mathrm{~K} \alpha$ and $\beta$ isoforms are needed for full Ins $\mathrm{P}_{3}$ formation and $\mathrm{Ca}^{2+}$ signal generation, thus suggesting that both activities are necessary for optimal activation of the key effector enzyme of GPVI, PLCY2. Detailed $\mathrm{Ca}^{2+}$ measurements in the presence of extracellular EGTA and $\mathrm{CaCl}_{2}$ pointed to a marked contribution of $\mathrm{Pl}_{3} \mathrm{~K}$ to $\mathrm{Ca}^{2+}$ mobilization from intracellular stores, which is a direct readout of PLC, and not to a (store-independent) effect on $\mathrm{Ca}^{2+}$ entry. Hence, the earlier suggested regulation of $\mathrm{Ca}^{2+}$ entry by $\mathrm{Pl}_{3} \mathrm{~K}$ or its product $\mathrm{PI}(3,4,5) \mathrm{P}_{3}(23,24)$ seems to be secondary to the effect of $\mathrm{Pl}_{3} \mathrm{~K}$ regulation of $\mathrm{Ca}^{2+}$ store depletion. 
Non-redundant roles of phosphoinositide 3-kinase isoforms $\alpha$ and $\beta$ in glycoprotein $\mathrm{V}$-induced platelet signaling and thrombus formation

Several observations point to a non-redundant contribution of $\mathrm{Pl}_{3} \mathrm{~K} \alpha$ and $\beta$ isoforms in GPVI-induced platelet activation. These include the inhibitory effects on human platelets of a panel of compounds with high affinity to $\mathrm{Pl} 3 \mathrm{~K} \alpha$ (PIK-75, $\mathrm{Pl}-103, \mathrm{YM}-024$ ) or to $\mathrm{Pl} 3 \mathrm{~K} \beta$ (TGX-221 and also $\mathrm{PI}$-103). Other support comes from studies with mouse platelets, which responded similarly to the isoform-specific inhibitors as did human platelets. Furthermore, we found that mouse platelets lacking p85 $\alpha$ were strikingly impaired in collagen-induced $\mathrm{Ca}^{2+}$ signaling and thrombus formation. These $\mathrm{p} 85 \alpha^{-/-}$platelets are fully depleted in p110 $\alpha$ but still express residual $p 85 \beta$ and $p 110 \beta$ (11). However, treatment of these platelets with TGX-221 did not further suppress $\mathrm{Ca}^{2+}$ signaling and downstream responses such as PS exposure, although this compound did insignificantly reduce $\mathrm{p} 85 \alpha^{-\gamma_{-}}$platelet deposition under flow. This suggested a function of the residual $p 110 \beta$ in ADP-dependent platelet aggregate formation.

In human platelets, inhibition of $\mathrm{Pl}_{3} \mathrm{~K} Y$ (AS-252424) failed to affect the GPVI-induced $\mathrm{Ca}^{2+}$ response, while inhibition of $\mathrm{PI}_{3} \mathrm{~K} \delta$ (IC-87114) caused a minor reduction, such in contrast to the marked effect of $\mathrm{Pl}_{3} \mathrm{~K} \alpha / \beta$ inhibition. Similarly, mouse platelets which were deficient in p110 $\gamma$ or p110 $\delta$ showed undiminished activation, when stimulated with GPVI agonist under conditions where autocrine stimulators were blocked. These results are supported by data from others, showing that deficiency in murine p110y (19) or p110 $\delta$ (26) has no more than a modest role in GPVI-induced platelet activation.

The role of $\mathrm{Pl}_{3} \mathrm{~K} \alpha$ and $\beta$ isoforms was particularly clear in measurements of GPVIdependent thrombus formation, where platelets were flowed over collagen at a defined physiological shear rate. Blocking or deficiency in $\mathrm{Pl}_{3} \mathrm{~K} \alpha / \beta$ resulted in suppression of $\mathrm{Ca}^{2+}$ rises and PS exposure of platelets adhered to collagen, as well as in reduced formation of aggregate formation. For both human and mouse blood, it is known that these responses fully rely on GPVI signaling via Src family kinases to LAT and PLCY2 $(34,38,46)$. Hence, the present findings support a concept that full PLCY2-evoked activity is required for optimal platelet activation and thrombus formation under flow. Earlier, it was established that at the same flow conditions different populations of platelets assemble into aggregates or expose PS (38). While this heterogeneity in platelet responses was maintained with $\mathrm{Pl}_{3} \mathrm{~K} \alpha / \beta$ inhibition or absence, the present results also suggest that the reduced PS exposure in inhibited platelets is a direct consequence of the reduced $\mathrm{Ca}^{2+}$ signal of those platelets that are adhered to collagen.

Earlier, we and others have shown that two $\mathrm{Pl}_{3} \mathrm{~K}$ isoforms, namely $\mathrm{Pl}_{3} \mathrm{~K} \beta$ and $\gamma_{1}$, are implicated in ADP/P2 $Y_{12}$-induced integrin $\alpha$ llb $\beta_{3}$ activation and subsequent thrombus stabilization $(17,19,20)$. Recently, these observations were extended to in vivo thrombosis models, where wortmannin treatment of p110y deficient mice was found to cause a dramatic defect in initial thrombus growth following vascular damage (21). The present data shed a new light on these in vivo observations, since it now appears that both collagen- and ADP-dependent platelet activation processes are affected by $\mathrm{Pl}_{3} \mathrm{~K}$ inhibition with a likely key role of the $\mathrm{PI} / 3 \mathrm{~K} \alpha / \beta$ isoforms.

There is a growing body of evidence that in many cell systems individual $\mathrm{Pl}_{3} \mathrm{~K}$ isoforms, although involved in specific cellular functions, show functional redundancy, e.g. as noted for the additive contribution of $\mathrm{Pl}_{3} \mathrm{~K} \beta$ and $\gamma$ in complement 5 a-stimulated macrophages (47). So far, non-redundancy in $\mathrm{Pl}_{3} \mathrm{~K}$ function has been described only in particular cases. For example, in neutrophils producing inflammatory reactive oxygen species, both $\mathrm{Pl}_{3} \mathrm{~K} \gamma$ and $\delta$ isoforms contribute to $\mathrm{PI}(3,4,5) \mathrm{P}_{3}$ formation, but in this case the role of $\mathrm{Pl}_{3} \mathrm{~K} \gamma$ may be prior to that of 
$\mathrm{Pl}_{3} \mathrm{~K} \delta(31,48)$. In comparison to platelets, this is reminiscent of the early contribution of $\mathrm{Pl}_{3} \mathrm{~K} \alpha / \beta$ in GPVI stimulation, and the later involvement of $\mathrm{Pl}_{3} \mathrm{~K} \beta / \gamma$ in responses to autocrine produced ADP.

In the classical scheme of immunoglobulin receptor-linked PLC $Y$ activity, as proposed for lymphocytes, accumulation of $\mathrm{PI}(3,4,5) \mathrm{P}_{3}$ in the plasma membrane may function as an anchoring site for the N-terminal pleckstrin homology domain of PLC $\gamma$ isoforms $(18,49)$. This scheme can be applicable to GPVI-stimulated platelets as well. Given the high turnover of phosphoinositides in the platelet plasma membrane, we hypothesize that the stimulation and anchoring of $\mathrm{PLC} \gamma_{2}$ requires a threshold elevation of $\mathrm{PI}(3,4,5) \mathrm{P}_{3}$ that can only be achieved by simultaneous activity of $\mathrm{Pl}_{3} \mathrm{~K} \alpha$ and $\beta$. In other words, activation of either $\mathrm{Pl}_{3} \mathrm{~K} \alpha$ or $\beta$ alone may be insufficient for PLC $\gamma_{2}$ targeting to membrane via its $\mathrm{PH}$ domain. It is conceivable that this is a consequence of the need of $\mathrm{PLC} \mathrm{Y}_{2}$ to compete with other $\mathrm{PH}$-domain containing signaling proteins such as Akt isoforms. Such a mechanism would provide an attractive, but not the only, explanation for the identified non-redundant contribution to GPVI-induced signaling of both $\mathrm{Pl}_{3} \mathrm{~K}$ isoforms. One possible scenario is that one isoform may produce an initial small amount of $\mathrm{PI}(3,4,5) \mathrm{P}_{3}$, which in turn promotes activation of the other isoform through $\mathrm{PI}(3,4,5) \mathrm{P}_{3}$ regulation of $\mathrm{p} 85$ subunits. Future studies examining temporal signaling by individual $\mathrm{Pl}_{3} \mathrm{~K}$ isoforms, as previously performed in neutrophils (31), will be needed to address this issue. The enhancing effect of $\mathrm{PI}_{3} \mathrm{~K}$ activity on PLCY2 is in contrast to the essential contribution of Src family kinases to GPVIinduced activation, as this is apparent from the complete abrogation of GPVI-induced $\mathrm{Ca}^{2+}$ rises by Src family kinase inhibition (51).

The literature contains solid evidence that platelet Rap1b is activated in a $\mathrm{Pl}_{3} \mathrm{~K}$-dependent manner, in particular after occupation of Gi-coupled receptors such as the $\mathrm{P}_{2} \mathrm{Y}_{12}$ receptor for $\operatorname{ADP}(27,28)$. By using autocrine stimulation inhibitors, where the $\mathrm{P}_{2} \mathrm{Y}_{12}$-dependent component was blocked, we could identify - for both human and mouse platelets - the existence of a direct GPVI-stimulated and $\mathrm{Pl}_{3} \mathrm{~K}$-dependent component of Rapıb activation. Use of the isoform specific inhibitors revealed involvement of $\mathrm{Pl}_{3} \mathrm{~K} \alpha / \beta$ in this activation of Rap1b. However, the prominent suppressive effect of $\mathrm{Pl}_{3} \mathrm{~K}$ inhibitors on $\alpha$ llb $\beta_{3}$ activation and aggregation seen in $\mathrm{Rap}_{1} \mathrm{~b}^{-/}$ platelets indicates that the $\mathrm{PI}_{3} \mathrm{~K}$ isoforms contribute to GPVI-induced integrin activation also through a pathway that is independent of Rapıb. This pathway may involve $\mathrm{Pl} 3 \mathrm{~K} \alpha / \beta$-dependent stimulation of $\mathrm{PLC} \mathrm{C}_{2}$, which can contribute to the $\mathrm{Ca}^{2+}$ - and protein kinase $\mathrm{C}$-mediated activation of $\alpha$ llb $\beta_{3}$.

$\mathrm{Pl}_{3} \mathrm{~K} \alpha$ is the most frequently mutated kinase in human cancer. Hence, this isoform has raised great interest as a target for novel anti-tumor drugs, some of which are currently in earlystage clinical trials (50). Given the likely important function of GPVI in (experimental) thrombosis, the present data suggest another important application for $\mathrm{PI}_{3} \mathrm{~K} \alpha$ and $\mathrm{Pl}_{3} \mathrm{~K} \beta$ inhibitors, namely in antiplatelet therapy and arterial thrombosis. In this context, it is relevant to note that arterial thrombosis is a known companion of some cancers. The availability of selective pharmacological agents against specific isoforms may thus offer new potential approaches for prevention of these diseases.

Note: While this paper was in revision, other authors have also reported that $\mathrm{PIK}_{3} \beta$ plays an essential role in GPVI-induced activation of mouse platelets (53). 


\section{Acknowledgements}

Non-redundant roles of phosphoinositide 3-kinase isoforms $\alpha$ and $\beta$ in glycoprotein VI-induced platelet signaling and thrombus formation

We thank Dr. A. van Montfoort for performing preliminary experiments. We acknowledge the Baker Heart Research Institute (Melbourne, Australia) for the kind gift of $\mathrm{Pl}_{3} \mathrm{~K}$ isoform inhibitors. This work was supported by grants from the EU (Marie Curie EST 2005-020706), the Netherlands Heart Foundation (2002-B014), and the Netherlands Organization for Scientific Research (NWO 11.400.0076).

\section{References}

$1 \quad$ Nieswandt B \& Watson SP. Platelet-collagen interaction: Is GPVI the central receptor? Blood 102, 449-61 (2003).

2 Watson SP \& Gibbins JM. Collagen receptor signaling in platelets: Extending the role of the ITAM. Immunol Today 19, 260-4 (1998).

3 Heemskerk JW, Kuijpers MJ, Munnix IC, \& Siljander PR. Platelet collagen receptors and coagulation. A characteristic platelet response as possible target for antithrombotic treatment. Trends Cardiovasc Med 15, 86-92 (2005).

4 Jackson SP. The growing complexity of platelet aggregation. Blood 109, 5087-95 (2007).

5 Jandrot-Perrus $\mathrm{M}$, Lagrue $\mathrm{AH}$, Okuma $\mathrm{M}$, \& Bon C. Adhesion and activation of human platelets in duced by convulxin involve glycoprotein $\mathrm{VI}$ and integrin $\alpha_{2} \beta_{1}$. J Biol Chem 272, 27035-41 (1997).

6 Knight CG, Morton LF, Onley DJ, Peachey AR, Ichinohe T, Okuma M, Farndale RW, \& Barnes MJ. Collagen-platelet interaction: Gly-Pro-Hyp is uniquely specific for platelet GPVI and mediates platelet activation by collagen. Cardiovasc Res 41, 450-7 (1999).

7 Siljander P, Farndale RW, Feijge MAH, Comfurius P, Kos S, Bevers EM, \& Heemskerk JW. Platelet adhesion enhances the glycoprotein VI-dependent procoagulant response: involvement of p38 MAP kinase and calpain. Arterioscler Thromb Vasc Biol 21, 618-27 (2001).

8 Gibbins JM, Briddon S, Shutes A, van Vugt MJ, van de Winkel JG, Saito T, \& Watson SP. The p85 subunit of phosphatidylinositol 3 -kinase associates with the Fc receptor $\gamma$-chain and linker for activator of T cells in platelets stimulated by collagen and convulxin. J Biol Chem 273, 34437-43 (1998).

9 Lagrue AH, Francischetti IM, Guimaraes JA, \& Jandrot-Perrus M. Phosphatidylinositol 3-kinase and tyrosine-phosphatase activation positively modulate convulxin-induced platelet activation. Comparison with collagen. FEBS Lett 448, 95-100 (1999).

10 Pasquet JM, Bobe R, Gross B, Gratacap MP, Tomlinson MG, Payrastre B, \& Watson SP. A collagenrelated peptide regulates phospholipase $C_{\gamma} 2$ via phosphatidylinositol 3 -kinase in human platelets. Biochem J 342, 171-7 (1999).

11 Watanabe N, Nakajima H, Suzuki H, Oda A, Matsubara Y, Moroi M, Terauchi Y, Kadowaki T, Suzuki H, Koyasu S, Ikeda Y, \& Handa M. Functional phenotype of phosphoinositide 3-kinase p85 $\alpha$-null platelets characterized by an impaired response to GPVI stimulation. Blood 102, 541-8 (2003).

12 Stephens L, Williams R, \& Hawkins P. Phosphoinositide 3-kinases as drug targets in cancer. Curr Opin Pharmacol 5, 357-65 (2005).

13 Ruckle T, Schwarz MK, \& Rommel C. PI3Ky inhibition: towards an 'aspirin of the 21st century'? Nat Rev Drug Discov 5, 903-18 (2006).

14 Ali K, Bilancio A, Thomas M, Pearce W, Gilfillan AM, Tkaczyk C, Kuehn N, Gray A, Giddings J, Peskett E, Fox R, Bruce I, Walker C, Sawyer C, Okkenhaug K, Finan P, \& Vanhaesebroeck B. Essential role for the $110 \delta$ phosphoinositide 3-kinase in the allergic response. Nature 431, 1007-11 (2004).

15 Rittenhouse SE. Phosphoinositide 3-kinase activation and platelet function. Blood 88, 4401-14 (1996).

16 Vanhaesebroeck B \& Waterfield MD. Signaling by distinct classes of phosphoinositide 3-kinases. Exp Cell Res 253, 239-54 (1999).

17 Jackson SP, Schoenwaelder SM, Goncalves I, Nesbitt WS, Yap CL, Wright CE, Kenche V, Anderson KE, Dopheide SM, Yuan Y, Sturgeon SA, Prabaharan H, Thompson PE, Smith GD, Shepherd PR, Daniele N, Kulkarni S, Abbott B, Saylik D, Jones C, Lu L, Giuliano S, Hughan SC, Angus JA, Robertson AD, \& Salem HH. PI 3-kinase p110ß: a new target for antithrombotic therapy. Nat Med 11, 507-14 (2005).

18 Schlessinger J. Cell signaling by receptor tyrosine kinases. Cell 103, 211-25 (2000).

19 Hirsch E, Bosco O, Tropel P, Laffargue M, Calvez R, Altruda F, Wymann M, \& Montrucchio G. Resistance to thromboembolism in $\mathrm{Pl}_{3} \mathrm{~K} \gamma$-deficient mice. FASEB J 15, 2019-21 (2001). 
Cosemans JM, Munnix IC, Wetzker R, Heller R, Jackson SP, \& Heemskerk JW. Continuous signaling via $\mathrm{Pl}_{3} \mathrm{~K}$ isoforms $\beta$ and $\gamma$ is required for platelet ADP receptor function in dynamic thrombus stabilization. Blood 108, 3045-52 (2006).

21 Schoenwaelder SM, Ono A, Sturgeon S, Chan M, Mangin P, Maxwell MJ, Turnbull S, Mulchandani M, Anderson K, Kauffenstein G, Rewcastle GW, Kendall J, Gachet C, Salem HH, \& Jackson SP. Identification of a unique co-operative $\mathrm{Pl}_{3} \mathrm{~K}$ signaling mechanism regulating integrin $\alpha \mathrm{llb} \beta_{3}$ adhesive function in platelets. J Biol Chem 282, 28648-58 (2007).

22 Lecut C, Schoolmeester A, Kuijpers MJ, Broers JL, van Zandvoort MA, Vanhoorelbeke K, Deckmyn H, Jandrot-Perrus $M, \&$ Heemskerk JW. Principal role of glycoprotein VI in $\alpha_{2} \beta_{1}$ and $\alpha_{l} \mathrm{lb} \beta_{3}$ activation during collagen-induced thrombus formation. Arterioscler Thromb Vasc Biol 24, 1727-33 (2004).

23 Pasquet JM, Quek L, Stevens C, Bobe R, Huber M, Duronio V, Krystal G, \& Watson SP. Phosphatidyl inositol 3,4,5-trisphosphate regulates $\mathrm{Ca}^{2+}$ entry via Btk in platelets and megakaryocytes without increasing phospholipase C activity. EMBO J 19, 2793-802 (2000).

24 Lu PJ, Hsu AL, Wang DS, \& Chen CS. Phosphatidylinositol 3,4,5-trisphosphate triggers platelet aggregation by activating $\mathrm{Ca}^{2+}$ influx. Biochemistry 37, 9776-83 (1998).

25 Woulfe $D$, Jiang $H$, Morgans A, Monks R, Birnbaum M, \& Brass LF. Defects in secretion, aggregation and thrombus formation in platelets from mice lacking Akt2. J Clin Invest 113, 441-50 (2004).

26 Senis YA, Atkinson BT, Pearce AC, Wonerow P, Auger JM, Okkenhaug K, Pearce W, Vigorito E, Vanhaesebroeck B, Turner M, \& Watson SP. Role of the $\mathrm{p}_{110} \delta \mathrm{PI}_{3} \mathrm{~K}$ in integrin and ITAM receptor signalling in platelets. Platelets 16, 191-202 (2005).

27 Lova P, Paganini S, Hirsch E, Barberis L, Wymann M, Sinigaglia F, Balduini C, \& Torti M. A selective role for phosphatidylinositol 3,4,5-trisphosphate in the Gi-dependent activation of platelet Rap1B. J Biol Chem 278, 131-8 (2003).

28 Woulfe D, Jiang H, Mortensen R, Yang J, \& Brass LF. Activation of Rapib by Gi family members in platelets. J Biol Chem 277, 23382-9o (2002).

29 Suzuki H, Terauchi Y, Fujiwara M, Aizawa S, Yazaki Y, Kadowaki T, \& Koyasu S. Xid-like immunedeficiency in mice with disruption of the $p 85 \alpha$ subunit of phosphoinositide 3-kinase. Science 283, 3902 (1999).

30 Chrzanowska-Wodnicka M, Smyth SS, Schoenwaelder SM, Fischer TH, \& White GC, 2nd. Rap1b is required for normal platelet function and hemostasis in mice. J Clin Invest 115, 680-7 (2005).

31 Condliffe AM, Davidson K, Anderson KE, Ellson CD, Crabbe T, Okkenhaug K, Vanhaesebroeck B, Turner M, Webb L, Wymann MP, Hirsch E, Ruckle T, Camps M, Rommel C, Jackson SP, Chilvers ER, Stephens LR, \& Hawkins PT. Sequential activation of class IB and class IA PI $3 \mathrm{~K}$ is important for the primed respiratory burst of human but not murine neutrophils. Blood 106, 1432-40 (2005).

32 Kuijpers MJ, Schulte V, Bergmeier WG, Lindhout T, Brakebusch C, Offermanns S, Fässler R, Heemskerk JW, \& Nieswandt B. Complementary roles of platelet glycoprotein VI and $\alpha_{2} \beta_{1}$ integrin in collagen-dependent thrombus formation. FASEB J 17, 685-7 (2003).

33 Maxwell MJ, Yuan Y, Anderson KE, Hibbs ML, Salem HH, \& Jackson SP. SHIP1 and Lyn kinase negatively regulate integrin $\alpha$ llb $\beta 3$ signaling in platelets. J Biol Chem 279, 32196-204 (2004).

34 Siljander PR, Munnix IC, Smethurst PA, Deckmyn H, Lindhout T, Ouwehand WH, Farndale RW, \& Heemskerk JW. Platelet receptor interplay regulates collagen-induced thrombus formation in flowing human blood. Blood 103, 1333-41 (2004).

35 Feijge MA, van Pampus EC, Lacabaratz-Porret C, Hamulyak K, Lévy-Toledano S, Enouf J, \& Heemskerk JW. Inter-individual varability in $\mathrm{Ca}^{2+}$ signalling in platelets from healthy volunteers, relation with expression of endomembrane Ca ${ }^{2+}$-ATPases. Br J Haematol 102, 850-9 (1998).

36 Heemskerk JW, Feijge MA, Henneman L, Rosing J, \& Hemker HC. The $\mathrm{Ca}^{2+}$-mobilizing potency of $\alpha-$ thrombin and thrombin-receptor-activating peptide on human platelets. Eur J Biochem 249, 547-55 (1997).

37 Cauwenberghs S, Feijge MA, Theunissen E, Heemskerk JW, van Pampus EC, \& Curvers J. Novel methodology for the assessment of platelet transfusion therapy by measuring increased thrombus for mation and thrombin generation. Br J Haematol 136, 480-90 (2007).

38 Munnix IC, Strehl A, Kuijpers MJ, Auger JM, van der Meijden PE, van Zandvoort MA, oude Egbrink

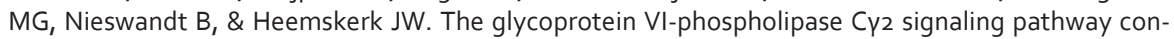
trols thrombus formation induced by collagen and tissue factor in vitro and in vivo. Arterioscler Thromb Vasc Biol 25, 2673-8 (2005).

39 Auger JM, Kuijpers MJ, Senis YA, Watson SP, \& Heemskerk JW. Adhesion of human and mouse platelets to collagen under shear: A unifying model. FASEB J 19, 825-7 (2005). 
Heemskerk JW, Willems GM, Rook MB, \& Sage SO. Ragged spiking of free calcium in ADP-stimulated human platelets: Regulation of puff-like calcium signals in vitro and ex vivo. J Physiol 535, 625-35 (2001).

41 Chaussade C, Rewcastle GW, Kendall JD, Denny WA, Cho K, Gronning LM, Chong ML, Anagnostou $\mathrm{SH}$, Jackson SP, Daniele N, \& Shepherd PR. Evidence for functional redundancy of class IA $\mathrm{PI}_{3} \mathrm{~K}$ isoforms in insulin signalling. Biochem J 404, 449-58 (2007).

Savage B, Ginsberg MH, \& Ruggeri ZM. Influence of fibrillar collagen structure on the mechanisms of platelet thrombus formation under flow. Blood 94, 2704-15 (1999).

43 Nesbitt WS, Mangin P, Salem HH, \& Jackson SP. The impact of blood rheology on the molecular and cellular events underlying arterial thrombosis. J Mol Med 84, 989-95 (2006).

44 Terauchi Y, Tsuji Y, Satoh S, Minoura H, Murakami K, Okuno A, Inukai K, Asano T, Kaburagi Y, Ueki K, Nakajima H, Hanafusa T, Matsuzawa Y, Sekihara H, Yin Y, Barrett JC, Oda H, Ishikawa T, Akanuma Y, Komuro I, Suzuki M, Yamamura K, Kodama T, Suzuki H, Yamamura K, Kodama T, Suzuki H, Koyasu S, Aizawa S, Tobe K, Fukui Y, Yazaki Y, \& Kadowaki T. Increased insulin sensitivity and hypoglycaemia in mice lacking the p85 $\alpha$ subunit of phosphoinositide 3-kinase. Nat Genet 21, 230-5 (1999).

45 Falet H, Barkalow KL, Pivniouk VI, Barnes MJ, Geha RS, \& Hartwig JH. Roles of SLP-76, phosphoinositide 3 -kinase, and gelsolin in the platelet shape changes initiated by the collagen receptor GPVI/FcRy- chain complex. Blood 96, 3786-92 (2000).

46 Nieswandt B, Bergmeier WG, Eckly A, Schulte V, Ohlmann P, Cazenave JP, Zirngibl H, Offermanns S, \& Gachet C. Evidence for cross-talk between glycoprotein $\mathrm{VI}$ and Gi-coupled receptors during collagen-induced platelet aggregation. Blood 97, 3829-35 (2001).

47 Guillermet-Guibert J, Bjorklof K, Salpekar A, Gonella C, Ramadani F, Bilancio A, Meek S, Smith AJ, Okkenhaug K, \& Vanhaesebroeck B. The p11o $\beta$ isoform of phosphoinositide 3-kinase signals down stream of G-protein-coupled receptors and is functionally redundant with p11op. Proc Natl Acad Sci USA 105, 8292-7 (2008).

48 Suire S, Condliffe AM, Ferguson GJ, Ellson CD, Guillou H, Davidson K, Welch H, Coadwell J, Turner M, Chilvers ER, Hawkins PT, \& Stephens L. GßYs and the Ras binding domain of p110y are both important regulators of $\mathrm{Pl}_{3} \mathrm{~K} \gamma$ signalling in neutrophils. Nat Cell Biol 11, 1303-9 (2006).

49 Scharenberg AM \& Kinet JP. Ptdlns-3,4,5-P3, a regulatory nexus between tyrosine kinases and sustained calcium signals. Cell 94, 5-8 (1998).

50 Zunder ER, Knight ZA, Houseman BT, Apsel B, \& Shokat KM. Discovery of drug-resistant and drugsensitizing mutations in the oncogenic isoform p110 $\alpha$. Cancer Cell 14, 107-8 (2008). 



\title{
Chapter 4
}

\author{
PKC a regulates platelet granule secretion and \\ thrombus formation in mice
}

Konopatskaya O, Gilio K, Harper MT, Zhao Y, Cosemans JM, Karim ZA, Whiteheart SW, Molkentin JD, Verkade P, Watson SP, Heemskerk, and JW Poole AW 


\begin{abstract}
Platelets are central players in the atherothrombosis of coronary artery disease. The protein kinase $C(P K C)$ family provides important intracellular mechanisms for regulation of platelet activity, and platelets express several members of this family including classical isoforms $\mathrm{PKC} \alpha$ and $\mathrm{PKC} \beta$ and novel isoforms $\mathrm{PKC} \delta$ and $\mathrm{PKC} \theta$. Here we use a genetic approach to demonstrate definitively the role played by $\mathrm{PKC} \alpha$ in regulating thrombus formation and platelet function. Thrombus formation in vivo was attenuated in $\mathrm{Prkca}^{-1-}$ mice, and PKC $\alpha$ was required for thrombus formation in vitro, although this $\mathrm{PKC}$ isoform did not regulate platelet adhesion to collagen. The ablation of in vitro thrombus formation in Prkca ${ }^{-1-}$ platelets was rescued by addition of ADP, consistent with the key mechanistic finding that dense-granule biogenesis and secretion depends upon PKC $\alpha$ expression. Furthermore, defective platelet aggregation in response to either collagen-related peptide or thrombin could be overcome by an increase in agonist concentration. Evidence of overt bleeding, including gastrointestinal and tail bleeding, was not seen in Prkcal- mice. In summary, the effects of PKC $\alpha$ ablation on thrombus formation and granule secretion may implicatet PKC $\alpha$ as a drug target for antithrombotic therapy.
\end{abstract}

\title{
Introduction
}

The protein kinase $\mathrm{C}$ (PKC) family comprises 10 isozymes grouped into 3 classes: conventional $\left(\alpha, \beta I, \beta I I, \gamma_{1}\right)$, novel $(\delta, \varepsilon, \eta / L, \theta)$ and atypical $(\zeta, L / \lambda)(1)$. PKCs have long been known to play major roles in a number of platelet processes (2), most importantly aggregation and secretion, where stimulation of platelets with diacyl glycerol (DAG) or phorbol ester can induce aggregation, and agonist-induced secretion can be prevented by broad spectrum pharmacological inhibition of PKC isoforms (3-6). Recently it has been shown pharmacologically that PKC isoforms may exert dual control role in thrombus formation by mediating secretion and integrin activation under flow, whilst also suppressing phosphatidylserine exposure and subsequent thrombin generation and coagulation (7).

At least four PKC isoforms $(\alpha, \beta, \delta, \theta)$ are expressed in human platelets (8-14) and it is becoming clear that each isoform may play different roles in platelet function. Using genetic and pharmacological approaches we have shown $\mathrm{PKC} \delta$ to play a negative role regulating filopodia formation and platelet aggregation in response to collagen through a functional interaction with the actin regulatory protein $\operatorname{VASP}(15,16)$. Using biochemical approaches PKC $\alpha$ has been identified as an essential factor in positively regulating alpha- and densegranule secretion in platelets (17) as well as platelet aggregation (18). We had recently shown, using biochemical and pharmacological approaches, that two tyrosine kinases Syk and Src physically and functionally interact with PKC $\alpha$ to regulate each other and cellular activities in platelets (19). It is now important that the function of PKC $\alpha$ in platelets is addressed definitively by a genetic approach.

Genetic and molecular approaches have revealed a wide range of roles for PKC $\alpha$ in other cell types. Cell proliferation, differentiation, apoptosis, motility and adhesion are all regulated by $\operatorname{PKC}(1,20)$ with consequent roles for $P K C \alpha$ in regulation of tumour progression. Although there is evidence that in some tumours PKC $\alpha$ may have a suppressor role 
$(21,22)$, in the majority of cases PKC $\alpha$ expression and activity are higher in tumours than in normal tissues $(23,24)$, and PKC $\alpha$ activity promotes a more aggressive phenotype in breast cancer cells with an increased metastatic potential (25-27). PKC $\alpha$ has therefore become a major target for therapeutic intervention in various cancers (28). In immune regulation PKC $\alpha$ mediates T cell-dependent interferon generation (29), and is also critical for T cell receptor down-regulation (30). Insulin signaling to $\mathrm{Pl}_{3} \mathrm{~K}$ and subsequently to glucose transport is enhanced in mice lacking $\mathrm{PKC} \alpha$ (31), suggesting a negative feedback role for $\mathrm{PKC} \alpha$ in metabolic processes. Finally, in the heart, $\mathrm{PKC} \alpha$ regulates cardiac contractility and $\mathrm{Ca}^{2+}$ handling in myocytes, and deficiency of $\mathrm{PKC} \alpha$ protects against heart failure induced by pressure overload, and against dilated cardiomyopathy (32).

In a recent elegant study reconstructing the signaling pathway regulating platelet integrin $\alpha$ llb $\beta_{3}$ in a nucleated cell system, it was shown that expression levels of PKC $\alpha$ equivalent to those found in platelets are required for activation of the integrin through the Rap1 pathway (33). This is in agreement with previous biochemical evidence suggesting an essential role for PKC $\alpha$ in regulating the integrin (18). Importantly also, genome-wide association analysis of coronary artery disease revealed a cluster of SNPs in the Prkca gene, with a maximal p-value association of just over $10^{-3}$ (SNP rs12600582) $(34,35)$. This analysis marks the gene as one requiring further phenotypic analysis. It was now essential to identify definitively, by a genetic approach, the role of PKC $\alpha$ in regulating platelet function and thrombus formation. Here we report that genetic ablation of PKC $\alpha$ in platelets reveals non-redundant roles in regulating platelet dense granule biogenesis and exocytosis, and in the control of thrombus formation in vitro and in vivo.

\section{Experimental procedures}

\section{Materials}

Collagen-related peptide (CRP) was from Prof Richard Farndale (University of Cambridge, UK). Thrombin, BSA, ADP, protein G-sepharose, fibrinogen were purchased from Sigma. Complete mini protease inhibitor tablets were from Roche Applied Science. Secondary HRP-conjugated anti-rabbit antibody were obtained from Santa Cruz Biotechnology Inc. SNAP23 (P-T95) antibody was a generous gift from Dr Paul Roche (NIH, Bethesda). JON/A (anti- $\alpha$ llb $\beta_{3}$ ) and WugEg (anti-P-selectin) antibody were from Emfret Analytics. Phycoerythrin (PE)-conjugated anti-CD41 antibody, and its isotype-matched control PEconjugated rat $\operatorname{Ig} G_{1}$, were from Serotec. Isoform-specific anti-PKC mouse monoclonal antibodies were from BD Biosciences. LY333531 was supplied by A.G. Scientific (Fluorochem Ltd), U46619 was from Alexis Biochemicals, Gö6976 was from Calbiochem and CHRONOLUME reagent came from Chrono-Log Corp. (Labmedics). The $\mathrm{P}_{2} \mathrm{Y}_{12}$ antagonist, ARC69931MX, was a kind gift from AstraZeneca. The $\mathrm{P}_{2} \mathrm{Y}_{1}$ antagonist MRS2500 was from Tocris Bioscience.

\section{Platelet preparation}

Mice were bred and maintained in the University of Bristol animal facility in accordance with UK Home Office regulations. Prkcal- mice were generated as previously described (32). 
Blood was drawn by cardiac puncture under terminal anaesthesia into acid citrate dextrose (20 mM citric acid/110 mM sodium citrate/5 mM glucose), 1:7 ratio v:v. Platelets were prepared as previously described (15). Briefly, blood was diluted with $250 \mu$ l of modified Tyrodes-HEPES buffer ( $134 \mathrm{mM} \mathrm{NaCl}, 0.34 \mathrm{mM} \mathrm{Na} 2 \mathrm{HPO}_{4}$, $2.9 \mathrm{mM} \mathrm{KCl}, 12 \mathrm{mM} \mathrm{NaHCO}$, 20 $\mathrm{mM} \mathrm{HEPES}, 5 \mathrm{mM}$ glucose, $1 \mathrm{mM} \mathrm{MgCl} 2, \mathrm{pH} 7.3$ ) and centrifuged at $180 \mathrm{~g}$ for $6 \mathrm{~min}$ at room temperature. Platelet-rich plasma (PRP) was removed and platelets were isolated by centrifugation at $550 \mathrm{~g}$ for $6 \mathrm{~min}$ in the presence of PGE1 (140 $\mathrm{nM}$ ) and indomethacin ( $10 \mu \mathrm{M})$. Pelleted platelets were resuspended to the required density in modified Tyrodes-HEPES buffer and rested for $30 \mathrm{~min}$ at $37^{\circ} \mathrm{C}$ in the presence of $10 \mu \mathrm{M}$ indomethacin prior to stimulation.

\section{Platelet aggregation}

Platelets were resuspended in Tyrodes-HEPES buffer at a final concentration $2 \times 10^{8}$ per ml. Platelets were preincubated with different inhibitors or vehicle solution (0.1\% Me2SO final concentration) for 10 minutes at $37^{\circ} \mathrm{C}$, and aggregation of agonist-stimulated platelets were monitored in an optical aggregometer (Chrono-Log, Labmedics) at $37^{\circ} \mathrm{C}$, with continuous stirring at $800 \mathrm{rpm}$.

\section{Flow cytometry}

Two-colour analysis of mouse platelet activation was conducted using phycoerythrinconjugated JON/A antibody that preferentially binds to the active form of $\alpha$ llb $\beta_{3}$ integrin and with FITC-conjugated CD62P (P-selectin) antibody. $25 \mu$ l of washed platelets $\left(4 \times 10^{7} / \mathrm{ml}\right.$ in Tyrode's-HEPES buffer supplemented with $1 \mathrm{mM} \mathrm{CaCl} 2$ and $0.35 \% \mathrm{BSA}$ ) were mixed with $10 \mu \mathrm{l}$ of antibody and subsequently stimulated either with $5 \mu \mathrm{g} / \mathrm{ml} \mathrm{CRP} \mathrm{or} 1 \mathrm{U} / \mathrm{ml}$ thrombin for $15 \mathrm{~min}$ at room temperature. The reaction was stopped by addition of $400 \mu \mathrm{l}$ ice-cold PBS and samples were analysed within $30 \mathrm{~min}$. For estimation of surface expression levels of total $\alpha$ llb $\beta_{3}$ integrin, $C_{41}$ was stained with PE-conjugated anti-CD41. Flow cytometry was performed on a FACSCalibur flow cytometer (BD Biosciences), using CellQuest version 3.1f software (BD Biosciences) and total 20,000 events per sample were collected.

\section{Measurement of ATP secretion}

ATP secretion was measured using CHRONO-LUME reagent according to the manufacturer's protocol. $5 \mu$ luciferase-luciferin were added directly to the cuvettes under stirring conditions (1000 rpm) and $5 \mu \mathrm{g} / \mathrm{ml} \mathrm{CRP} \mathrm{or} 1 \mathrm{U} / \mathrm{ml}$ thrombin were added to activate platelets for $1 \mathrm{~min}$. The luminescence intensity was measured at a luminescence setting of $0.05 \mathrm{x}$.

\section{Platelet spreading and adhesion assay}

Coverslips were coated with either $0.1 \mathrm{mg} / \mathrm{ml}$ fibrinogen or $50 \mu \mathrm{g} / \mathrm{ml} \mathrm{CRP} \mathrm{overnight,} \mathrm{fol-}$ lowed by non-specific blocking step with $2 \%$ BSA for 1 hour. Platelets were resuspended to $2 \times 10^{7} / \mathrm{ml}$ in modified Tyrodes-HEPES buffer and $500 \mu \mathrm{l}$ dispensed onto the coverslip mounted into a live-cell microscopy chamber. Adhesion and spreading of platelets was observed by differential interference contrast (DIC) microscopy with a wide-field micro- 
scope DM IRB attached to an ORCA ER camera (63x/1.40 NA oil objective) (Leica). 5 images in different random parts of the cover slip area were taken at 15,30 and $45 \mathrm{~min}$, and processed with OpenLab 4.03 (Improvision) and Adobe Photoshop software. The surface area and number of adherent platelets was estimated using Image J software.

\section{Immunoblotting}

For stimulated samples, $2 \times 10^{8} / \mathrm{ml}$ of washed platelets were activated with $5 \mu \mathrm{g} / \mathrm{ml}$ CRP or 1 unit/ml thrombin in the presence of $2 \mathrm{mM} \mathrm{CaCl}_{2}$ for $3 \mathrm{~min}$ and solubilised in Laemmli sample buffer. Proteins were resolved by electrophoresis in 9.5\% (for PKC isoform estimation) or $16 \%$ (for SNAP-23 Ser95 phosphorylation estimation) SDS-polyacrylamide gels. Samples were then transferred to polyvinylidene difluoride membranes, blocked with $10 \%$ bovine serum albumin, and subjected to immunoblotting with anti-PKC $\alpha, \beta, \delta$, or $\theta$ antibodies, SNAP- antibody or phospho-SNAP-23-Ser95 antibody.

\section{Thrombus formation under flow in vitro}

Flow-induced thrombus formation was assessed basically as described before (56). Heparin/PPACK-anticoagulated mouse blood was flowed over immobilised collagen through a parallel plate perfusion chamber, at a shear rate of $1000 \mathrm{~s}^{-1}$ for 4 minutes. Where indicated ADP ( $200 \mu \mathrm{M}$ in saline) was co-infused at a 10\% rate, immediately before the blood reached the flow chamber. For each perfusion surface, phase-contrast images from 10 random microscopic fields were collected. Surface coverage was analyzed using Image-Pro software version 4.1 (Silverspring, MD, USA). The average percentage area covered by adherent platelets was measured by automated setting of masks for the ranges of grey levels corresponding to the presence of platelets and thrombi. For each image, the (blinded) observer needed to approve the mask settings. Averaged pixel fractions of the masks from 10 images were considered as a best estimate of the surface area coverage with thrombi. Aggregate volume was also estimated by confocal laser scanning microscopy, using a BioRad 2100 multiphoton system, as described before (57). Platelet aggregates that formed on collagen ( 4 min, shear rate $1000 \mathrm{~s}^{-1}$ ) were post-labelled with FITC-anti-mouse CD62 mAb (1:100). Confocal stacks were recorded for measurement of the volume of individual platelet aggregates. Analysis of confocal images (grey level bit maps) and 3D reconstruction of images were with LaserPix software (Media Cybernetics).

\section{Detection of occult fecal blood}

The presence of occult fecal blood was detected by 'Hemdetect' (DIPRO med, supplied by Autogen Bioclear) on freshly obtained stool samples.

\section{Electron microscopy}

PRP was collected and spun at 590xg for $5 \mathrm{~min}$. From the platelet pellet the supernatant was removed and the pellet was fixed in $2.5 \%$ glutaraldehyde in $0.1 \mathrm{M}$ phosphate buffer $(\mathrm{pH} 7.4$, PB). The pellet was washed in PB and then incubated in $1 \%$ osmium tetra oxide in PB for 30 min. After washing in PB and de-ionised water the pellet was incubated in $3 \%$ uranyl acetate 
in de-ionised water for $30 \mathrm{~min}$. After a wash with de-ionised water the pellet was dehydrated in a graded series of ethanol (70, 80, 90, 96, 100, and 100\% each step $10 \mathrm{~min})$. After removal of the $100 \%$ ethanol, the pellet was incubated with pure Epon for $2 \mathrm{~h}$ at room temperature. Thereafter the Epon was replaced with fresh Epon and this was hardened overnight in a $60^{\circ} \mathrm{C}$ oven. Ultrathin counterstained sections were imaged at a Philips CM1oo equipped with a side-mount Megaview III camera (Olympus Soft Imaging Solutions).

To determine the dense and $\alpha$-granule content, total numbers of granules in equivalent-sized fields of view were counted. For each genotype, 25-30 randomly chosen fields of view were examined. All microscopic images were taken at the same magnification $19000 \mathrm{x}$ and the numbers of cells per field of view were equivalent between wild type and Prkcapreparations.

\section{Analysis of bleeding time}

Experiments were conducted on 25-35 g male and female mice. Mice were anesthetized (ketamine $75 \mathrm{mg} / \mathrm{kg}$; medetomidine $1 \mathrm{mg} / \mathrm{kg}$ intraperitoneally) and a transverse incision was made with a scalpel at a position where the diameter of the tail was 2.25 to $2.5 \mathrm{~mm}$. The tail was immersed in normal saline $\left(37^{\circ} \mathrm{C}\right)$ in a hand-held test tube and the time from incision to cessation of bleeding was recorded.

\section{Intravital microscopy of thrombus formation in vivo}

Intravital microscopy was performed essentially as described previously (44). Experiments were conducted on 25-30 $\mathrm{g}$ male Prkca ${ }^{-1-}$ mice and their littermate matched control wild types. All procedures were undertaken with United Kingdom Home Office approval in accordance with the Animals (Scientific Procedures) Act of 1986 (Project License No's: 40/2212, 40/2749 and 30/2386). Anesthesia was induced by intraperitoneal ketamine (100 $\mathrm{mg} / \mathrm{kg}$ Vetalar; Pharmacia and Upjohn Ltd) and $2 \%$ xylazine (20 mg/kg; Millpledge Pharmaceuticals). The left cremaster muscle was exteriorized and spread flat over an optically clear coverslip and continuously superfused. High speed intravital microscopy experiments were performed as previously described by Falati et al. (43).

Platelets were labelled fluorescently with Alexa-488-conjugated goat anti-rat antibody (Molecular Probes, Eugene, OR, USA) and rat anti-murine CD41 antibody (BD Biosciences Pharmingen) infused via a carotid cannula. Thrombi were induced in arterioles with a diameter of $25-35 \mu \mathrm{m}$ by a nitrogen ablation laser (Micropoint, Photonic Instruments), which introduced the laser through the microscope objective. Brightfield and fluorescent images were captured simultaneously for 4-5 min, and multiple thrombi were generated with a distance of at least $200 \mu \mathrm{m}$ between them and upstream to previous injuries. The integrated intensity value for the growing thrombus was plotted against time and peak size of thrombus and time taken to reach peak was determined. 


\section{Statistics}

Statistical analyses were carried on raw data using unpaired t-test and $p<0.05$ was considered statistically significant. Values are expressed as means \pm SEM. For all data " $n$ " is number of mice tested.

\section{Results}

Major role for $P K C \alpha$ in inside-out regulation of $\alpha / l b b_{3}$, but no significant role in outside-in signaling through this integrin

The PKC family of kinases has been implicated in agonist-induced $\alpha \mathrm{llb} \beta_{3}$ integrin activation (36), and although an elegant reconstruction study showed a critical role for PKC $\alpha$ in regulating the integrin (33), an involvement of PKC $\alpha$ in this event in platelets has not yet been addressed directly and definitively. Here we report, for the first time in platelet functional and thrombosis analysis, data from a mouse gene knockout lacking PKC $\alpha$. Supplementary Fig. 1 shows baseline data demonstrating lack of expression of $P K C \alpha$, but normal expression of other platelet PKCs $(\beta, \delta$, and $\theta)$ in Prkca ${ }^{-\gamma}$ platelets. Figure $1 \mathrm{~A}$ shows that both CRP (5 $\mu \mathrm{g} / \mathrm{ml}$ ) and thrombin ( 1 unit/ml) produced significantly greater $\alpha$ llb $\beta_{3}$ activation in wild type platelets compared to $\mathrm{Prkca}^{-1}$. This suggested that PKC $\alpha$ was a major regulator of inside-out signaling to $\alpha$ llb $\beta_{3}$, although the residual response in the absence of PKC $\alpha$ suggested a component independent of this kinase. By way of control, supplementary Fig. 2 shows no significant difference in surface expression of $\alpha$ llb (CD41) in Prkca $^{-/-}$vs wild type, irrespective of activation state of the platelets and irrespective of agonist (CRP or thrombin).
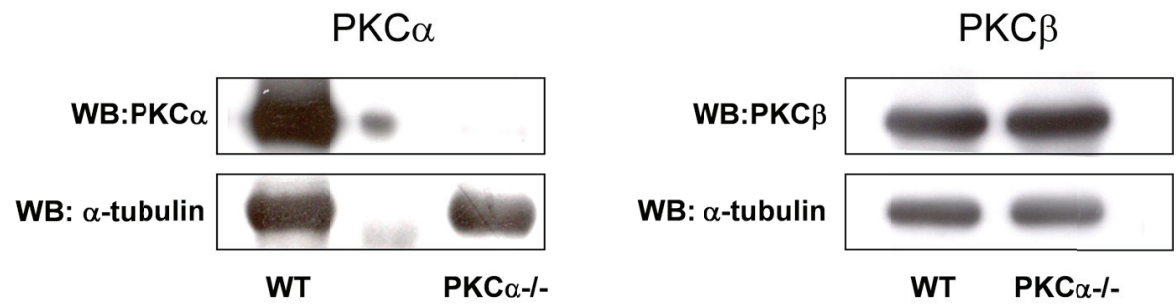

\section{$\operatorname{PKC} \delta$}

WB:PKC $\delta$

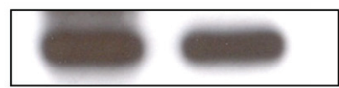

WB: $\alpha$-tubulin

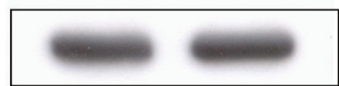

WT

PKC $\alpha-/-$
$\operatorname{PKC} \theta$

WB:PKC $\theta$

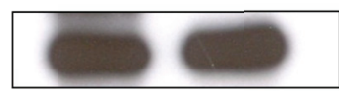

WB: $\alpha$-tubulin

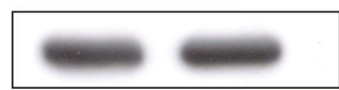

WT

PKC $\alpha-l-$

Supplementary Figure 1. Expression of PKC isoforms in Prkca ${ }^{-/}$mouse platelets. Whole cell lysates from Prkca $^{--}$mouse platelets were analysed by western blotting with anti-PKC $\alpha, P K C \beta, P K C \delta$, and PKC $\theta$ antibodies. PVDF membranes were stripped and re-probed with anti- $\alpha$-tubulin to confirm equal total protein loading. Blots shown are representative of three independent experiments. 
It has been shown recently that PKC $\beta$ and $\mathrm{PKC} \theta$ play critical roles regulating outside-in signaling from $\alpha$ llb $\beta_{3}(37,38)$. Hence in mouse platelets lacking either $\mathrm{PKC} \beta$ or $\mathrm{PKC} \theta$, cell spreading on fibrinogen-coated surfaces was ablated. Additionally, we had recently shown that absence of PKC $\delta$ caused platelets to spread on CRP- or collagen-coated surfaces with markedly more sustained generation of filopodia than wild type control platelets. It was important therefore to assess the role of PKC $\alpha$ in adhesion and spreading on either fibrinogen or CRP, and so we measured the number and surface area of adherent platelets by DIC microscopy over a 40 min period. Figure $1(B-D)$ shows that platelets lacking PKC $\alpha$ have no significant defect in their ability to spread on either fibrinogen-coated or CRP-coated surfaces, and importantly in the case of CRP there was no evident sustained development of filopodia. In addition, although Figures $1 \mathrm{C}$ and $1 \mathrm{D}$ show a trend towards decreased adhesion of platelets to both surfaces in Prkca $^{-1-}$ platelets, significance was achieved only at a single time point (30 min; 90.31 \pm 6.9 platelets/field of view in wild type compared to $62.18 \pm 5.6$ platelets/field of view in Prkca ${ }^{-1-}$ mice; mean \pm SEM, p<0.05) in platelets adherent to fibrinogen.

A

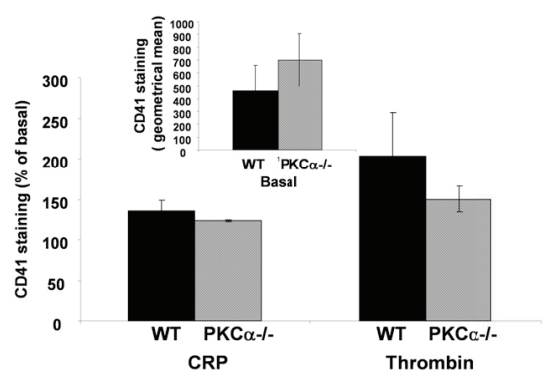

B
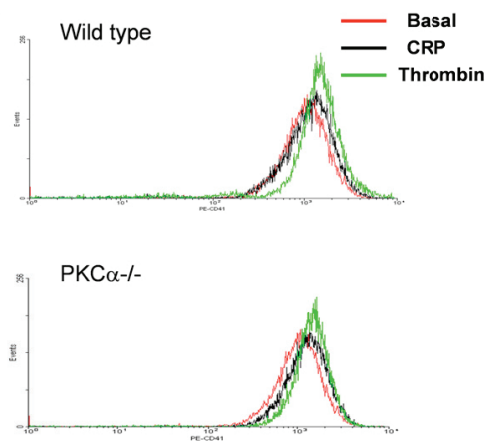

Supplementary Figure 2. Platelet surface expression of $\mathrm{CD}_{41} 1$ is not affected by $\mathrm{PKC} \alpha$ knock-down. Washed platelets from wild type (WT) or Prkcal- mice were labelled with PE-anti-CD 41 antibody, stimulated with CRP $(5 \mu \mathrm{g} / \mathrm{ml})$ or thrombin ( $1 \mathrm{unit} / \mathrm{ml}$ ) for $15 \mathrm{~min}$ and immunofluorescence intensity measured by flow cytometry. In (A), the data presented are geometric means as percentages of basal nonstimulated levels (error bar represents SEM, n=4). Inset panell shows geometric mean of fluorescence (arbitrary fluorescence units) of basal platelets from either wild type or Prkca ${ }^{-1-}$ mice. No difference was seen between wild type and Prkca ${ }^{-1}$ platelet expression of $\mathrm{CD}_{41}$, and $\mathrm{PKC} \alpha$ expression plays no role in regulating $\mathrm{CD}_{41}$ surface expression upon stimulation with thrombin or CRP. Data shown are mean \pm $\mathrm{SEM}, \mathrm{n}=4$. Additionally, no difference was seen between basal and CRP- or thrombin-stimulated platelets, from either wild type or Prkca ${ }^{-1-}$ mice, in staining with an isotype-matched control antibody (PEconjugated rat $\lg \mathrm{G}_{1}$; data not shown). In (B), representative frequency histograms are shown for $C_{41}$ expression from wild type (upper panel) and Prkcal- (lower panel) platelets, either basal or stimulated with CRP $(5 \mu \mathrm{g} / \mathrm{ml}$ ) or thrombin ( 1 unit/ml) for $15 \mathrm{~min}$ as indicated. Data are representative of 4 independent experiments. For color version, see page 186.

\section{$P K C \alpha$ is a major regulator of dense and $\alpha$-granule secretion}

Figure $2 \mathrm{~A}$ shows that Prkca $^{-1-}$ platelets also exhibit a marked reduction in CRP- and thrombin-induced ATP secretion relative to wild type controls (40.9 \pm 7.0 nM ATP in WT vs 8.9 \pm 3.4

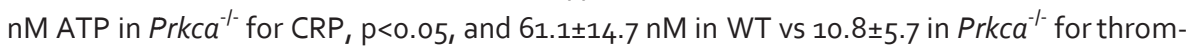


bin, $\mathrm{p}<0.05)$. The defect in granule secretion was not restricted to dense granules however but also involved regulation of $\alpha$-granules. Stimulation with CRP induced $\mathrm{P}$-selectin surface expression in wild type platelets which was significantly greater than that in Prkcal- platelets (WT: $411.9 \pm 145.3 \%$ over basal; Prkca ${ }^{-1}: 130.7 \pm 14.6 \%$ over basal). A similar marked knockdown of P-selectin exposure was observed after thrombin activation (Fig. 2B) $(705 \pm 235.7 \%$ in WT vs $113.9 \pm 5.5 \%$ in $\mathrm{KO}, \mathrm{p}<0.05)$, suggesting that $\mathrm{PKC} \alpha$ plays a major role in platelet $\alpha$-granule and dense granule release.

A

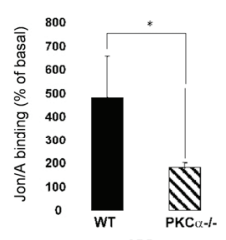

CRP
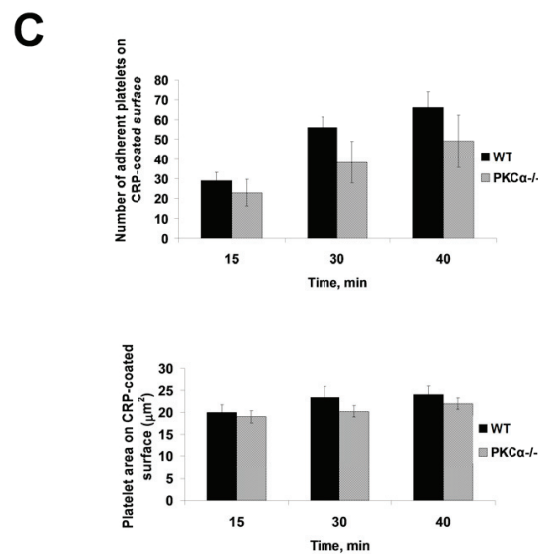

B

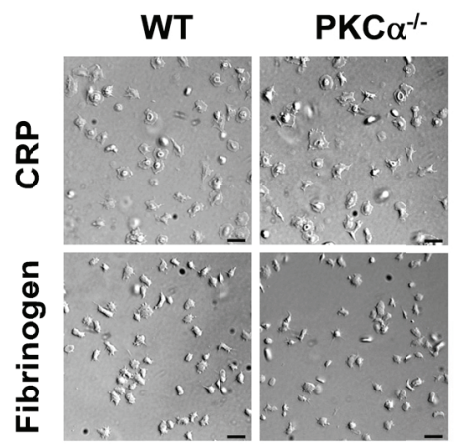

D

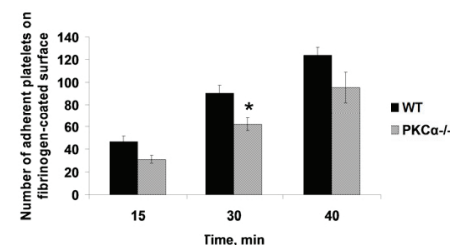

Figure 1. PKC $\alpha$ plays a major role regulating inside-out signaling to integrin $\alpha$ llb $\beta_{3}$, but no significant role in outside-in signaling or in adhesion to fibrinogen or collagen. (A) Washed platelets from wild type (WT) or Prkca ${ }^{-1-}$ mice were labelled with PE-antiallb $\beta_{3}$ antibody, stimulated with CRP (5 $\mu \mathrm{g} / \mathrm{ml}$ ) or thrombin ( 1 units $/ \mathrm{ml}$ ) for $15 \mathrm{~min}$ and immunofluorescence intensity measured by flow cytometry. The data presented are geometric means as percentages of basal non-stimulated levels (error bars represent SEM, $\left.\mathrm{n}=3,{ }^{*} \mathrm{p}<0.05\right)$. (B)-(D) Washed platelets from wild type (WT) or Prkca ${ }^{-/}$mice were added to CRP or fibrinogen-coated coverslips, and the levels of spreading/adherence were analysed. (B) Representative images of murine platelets $40 \mathrm{~min}$ after addition to CRP- or fibrinogen-coated surfaces as indicated. Images were taken under $63 x$ oil immersion and the bars represent $5 \mu \mathrm{m}$. (C) \& (D) Time course of WT and Prkca $^{-1-}$ platelet adherence (upper panel; number of platelets estimated per field of view at $63 x$ magnification) and spreading (as assessed by measurement of cell surface area, lower panel) on CRP-coated (C) and fibrinogen-coated (D) surface. Data shown are mean $\pm S E M, n=4$, * $p<0.05$. 
It has been demonstrated that SNAP-23, a member of SNARE traffic and fusion protein complex, undergoes PKC-dependent phosphorylation during agonist-stimulated platelet degranulation, and in vitro PKC $\alpha$ was one of the isoforms able to phosphorylate recombinant SNAP-23 (39). We therefore assessed the contribution of PKC $\alpha$ to phosphorylation of SNAP-23 on Ser95, the site reported to be phosphorylated following thrombin stimulation (40). Supplementary Fig. 3A shows a time course of phosphorylation of SNAP23 from wild type mouse platelets. This time course correlated well with release of ATP from those platelets stimulated with thrombin. We then showed that both CRP and thrombin activation led to a potent increase in SNAP-23 phosphorylation at Ser95, which was considerably, although not completely, inhibited by absence of PKC $\alpha$ (Supp. Fig. 3B). PKC $\alpha$ mediated phosphorylation of SNAP-23 may therefore play a role in regulating secretion or SNARE recycling.
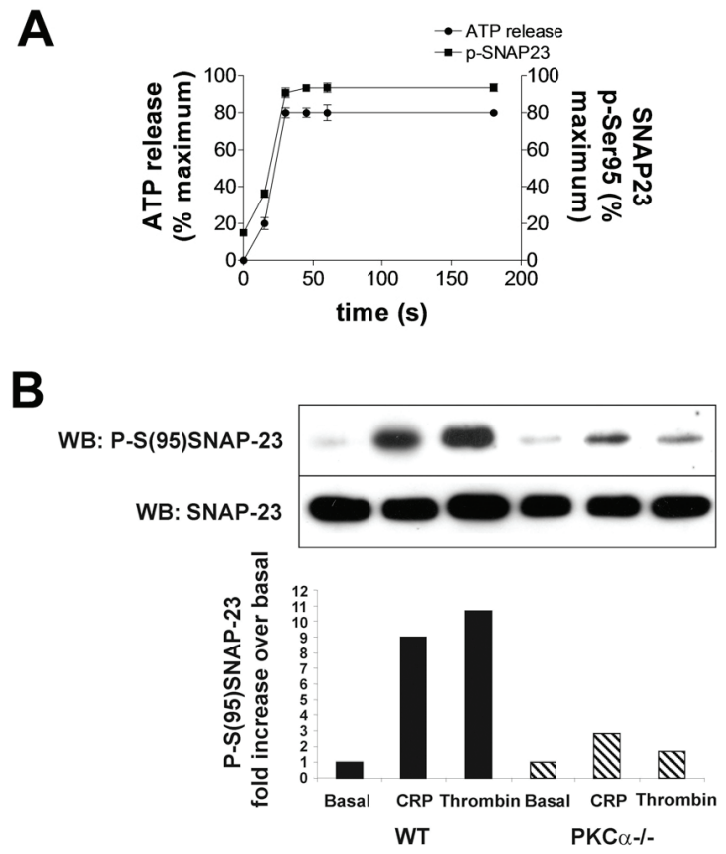

Supplementary Figure 3. SNAP-23 phosphorylation on Ser95 correlates with dense granule secretion and Prkca ${ }^{-/}$platelets. (A) Washed platelets from wild type mice were stimulated with thrombin ( 0.5 units $/ \mathrm{ml}$ ) and (i) secretion of ATP assessed by luminometry and (ii) phosphorylation of SNAP-23 on Serg5 assessed by immunoblotting and densitometry. Data shown are (i) increase in ATP concentration at time points after administration of thrombin, as shown, normalised to the maximum ATP release, and (ii) SNAP-23 pSer95 phosphorylation band density normalised to the maximum phosphorylation. Data are mean \pm SEM $(n=3)$. (B) Top panel: washed platelets from wild type (WT) or Prkca ${ }^{-/}$were stimulated with $5 \mathrm{\mu g} / \mathrm{ml}$ CRP or $1 \mathrm{unit} / \mathrm{ml}$ thrombin for $3 \mathrm{~min}$. Whole cells lysates were analysed for the expression of total SNAP-23 and phospho-SNAP-23-Ser95. Bottom panel: densitometric analysis of phospho-SNAP-23-Serg5 immunoblot. The immunoblots shown are representative of 3 independent experiments. 
It was possible that the secretory defect seen in Prkca $^{-1-}$ platelets may be as a result of a granule developmental problem. Transmission electron microscopy (TEM) analysis of platelet ultrastructure showed an abnormality in the number of dense granules in Prkcaplatelets, characterised by $\sim 3.7$-fold reduction compared to wild type platelets (Fig. 3). Dense granule biogenesis in megakaryocytes or peripheral distribution during proplatelet development could be the key points of regulation by $\mathrm{PKC} \alpha$, and this may additionally explain the major defect in dense granule secretion seen in Prkcal- platelets. TEM quantification of $\alpha$-granule numbers however did not detect any substantial difference between wild type and Prkca $^{-/-}$platelets, indicating that the defect in $\alpha$-granule secretion is due to an essential role played by PKC $\alpha$ in regulation of secretion of these granules, rather than their biogenesis.
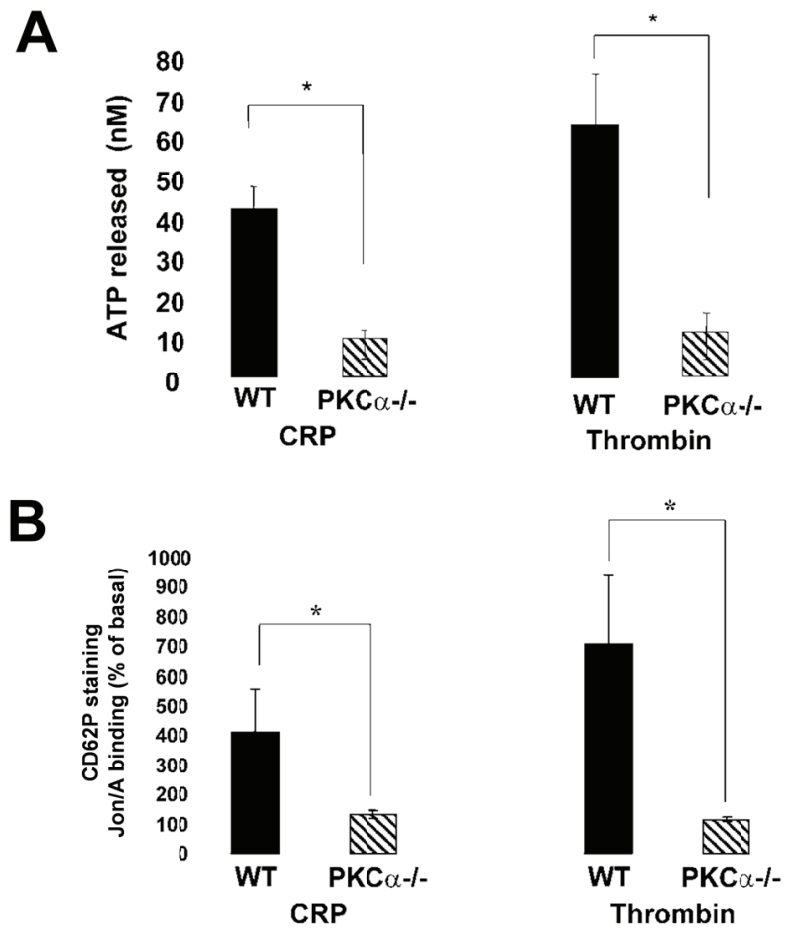

Figure 2. Key role for $P K C \alpha$ in secretion of dense and $\alpha$-granules. (A) Washed platelets from wild type (WT) or Prkca ${ }^{-/-}$mice were stimulated with collagen-related peptide (CRP, $\left.5 \mu \mathrm{g} / \mathrm{ml}\right)$ or thrombin $(0.25$ units $/ \mathrm{ml}$ ) and secretion of ATP assessed by luminometry. Data shown are maximal increase in ATP concentration, and are mean $\pm \operatorname{SEM}\left(n=3,{ }^{*} p<0.05\right)$. (B) Platelets from wild type (WT) or Prkca ${ }^{-1-}$ mice were labelled with FITC-C62P antibody, stimulated with CRP $(5 \mu \mathrm{g} / \mathrm{ml})$ or thrombin ( 1 units $/ \mathrm{ml})$ for 15 min and fluorescence intensity measured by flow cytometry. The data presented are geometric means as percentages of basal non-stimulated levels (error bars represent SEM, $n=3,{ }^{*} p<0.05$ ). 
Platelet aggregation deficit in Prkca ${ }^{-/}$platelets is rescued by exogenous ADP

It was now important to determine whether the deficits in secretion and activation of integrin $\alpha$ llb $\beta_{3}$ would translate into functional deficits in platelet aggregation and thrombus formation. Figure 4 shows concentration-response relationships for platelet aggregation activated by CRP (A) or thrombin (B). At submaximal concentrations of either agonist, absence of PKC $\alpha$ has a marked effect upon the aggregation response, such that the aggregation responses to $1.25 \mathrm{\mu g} / \mathrm{ml}$ CRP or $0.065 \mathrm{units} / \mathrm{ml}$ thrombin are almost ablated in Prkca ${ }^{-1}$ platelets. Importantly however, responses may be effectively rescued by addition of exogenous ADP $(10 \mu \mathrm{M})$, added simultaneously with CRP or thrombin. This indicates that the important functional deficit in Prkcal- $^{-/}$platelets is the lack of dense granule secretion. The effect upon aggregation was also relative, since higher concentrations of either CRP or thrombin were able to restore aggregations to wild-type values.

A

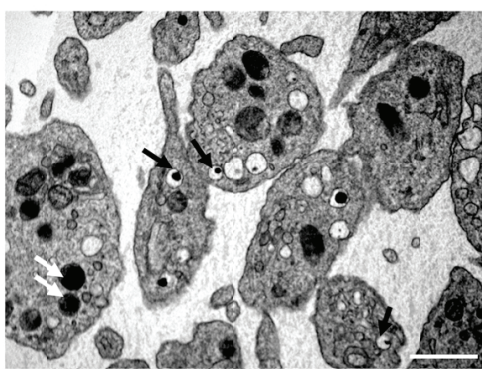

WT

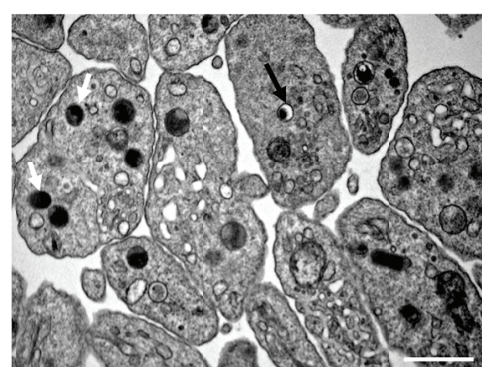

PKC $\alpha-/-$

B
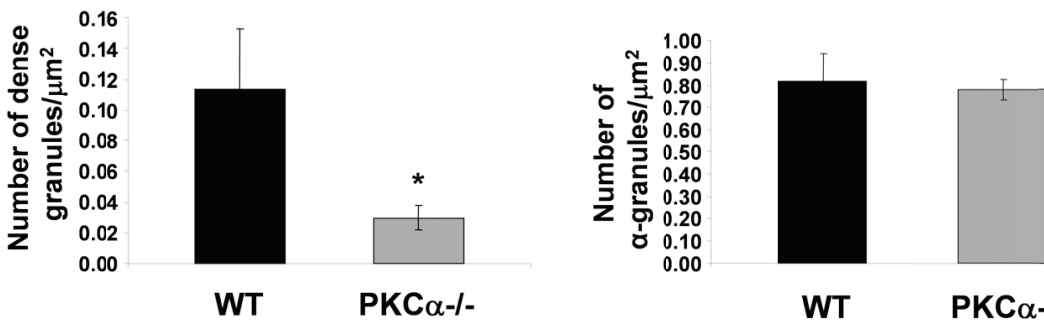

PKC $\alpha=/-$

Figure 3. PKC $\alpha$ regulates dense granule, but not $\alpha$-granule, biogenesis. Washed platelets from wild type (WT) or Prkca ${ }^{-1-}$ mice were examined by transmission electron microscopy, and the number of dense granules (black arrows) and $\alpha$-granules (white arrows) was quantified as described in Methods. For (A), images of wild type (left-hand panel) or Prkca $^{-\gamma_{-}}$(right-hand panel) platelets are representative of 3 independent experiments, and scale bar is $1 \mu \mathrm{m}$, magnification 19000x. For (B), dense granules (left-hand panel) and $\alpha$-granules (right-hand panel) were counted per field of view (25-30 fields of view per preparation), and shown as mean \pm SEM number of granules per $\mu \mathrm{m} 2(n=3, * p<0.05)$.

Pharmacological inhibition of PKCB reveals redundancy with $P K C \alpha$ for regulation of platelet aggregation

Given that higher concentrations of agonists are capable of overcoming the aggregation deficits seen in the absence of PKC $\alpha$, it was important to address whether there may be functional redundancy between PKC $\alpha$ and its closely related family member, PKC $\beta$. To 
assess this possibility we pre-incubated platelets with the inhibitor of classical PKC isoforms Gö6976 at its maximally effective concentration ( $1 \mu \mathrm{M})$, which completely abrogated CRPinduced aggregation both in wild type and $\mathrm{PrkCa}^{-/-}$mouse platelets (Fig. 5A). This confirmed an indispensable role for classical PKC isoforms in GPVI-mediated aggregation, and suggested a potential redundancy between $P K C \alpha$ and $P K C \beta$ in regulating aggregation. This redundancy was demonstrated by using the PKC $\beta$-selective inhibitor, LY333531 (ruboxistaurin), which at a maximally effective concentration (10 $\mu \mathrm{M})$ had no significant effect upon aggregation of wild type platelets to $C R P$, but was markedly inhibitory to this response in platelets lacking PKC $\alpha$ (Fig. 5B). This was paralleled for thrombin ( 0.5 units/ml), where 10 $\mu \mathrm{M}$ LY333531 had no effect upon wild type platelet aggregation response, but markedly inhibited the response in Prkcal- platelets (data not shown). Interestingly, for weaker agonists such as the thromboxane analogue $\mathrm{U}_{46619}$, aggregation was ablated in platelets lacking $\mathrm{PKC} \alpha$, although shape change in these platelets was unaffected (Fig. ${ }_{5} \mathrm{C}$ ). This is consistent with pharmacological data that show a critical role for PKC in platelet aggregation induced through the thromboxane receptor system (41). These data therefore suggested that, for weak agonists, PKC $\alpha$ plays an essential role, whereas redundancy between $\mathrm{PKC} \alpha$ and $\mathrm{PKC} \beta$ exists for strong agonists in regulating the platelet aggregation response.

A

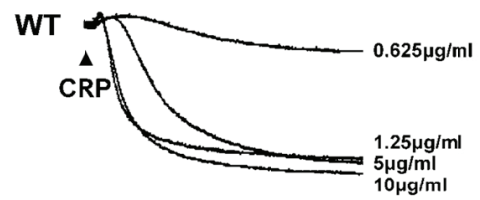

OD $10 \% \frac{}{1 \mathrm{~min}}$

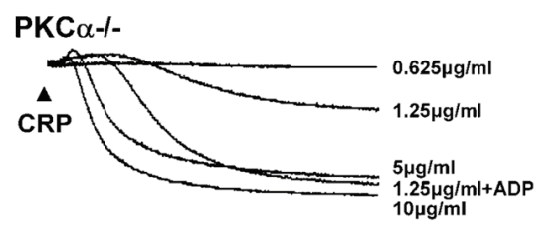

OD $10 \% \frac{}{1 \mathrm{~min}}$
B

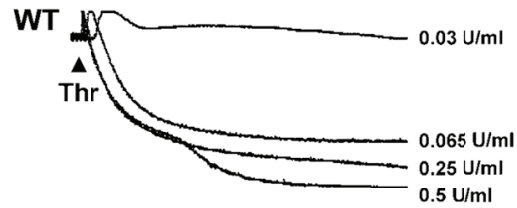

OD $10 \% \frac{L_{1} \min }{}$

PKC $\alpha-$

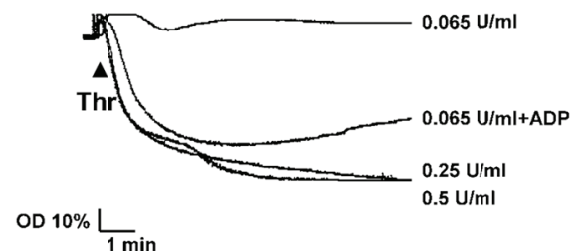

Figure 4. ADP rescues deficient aggregation responses to CRP or thrombin in Prkca ${ }^{-1-}$ platelets. Washed platelets from wild type (WT) or Prkca- mice were stimulated with various concentrations of collagen-related peptide (CRP) or thrombin with or without simultaneous addition of ADP (10 $\mu \mathrm{M})$ and aggregation assessed turbidimetrically. Aggregation traces shown are representative of 4 independent experiments.

\section{Ablation of $P K C \alpha$ markedly attenuates thrombus formation in vitro and in vivo}

Finally, it was important to determine the role played by PKC $\alpha$ in thrombus formation both in vitro and in vivo. Figure 6A (upper panels) shows a major role for PKC $\alpha$ in vitro in mediating this function, where the images reveal no reduction in primary adhesion of platelets to 
the collagen-coated surface, consistent with the absence of effect upon static adhesion shown in Fig. 1, but a marked reduction in aggregated platelet thrombus formation on top of the initially adherent cells. In order to confirm, in a quantitative manner, the lack of effect of PKC $\alpha$ ablation on primary adhesion, Fluo-4-labeled platelets were back-added to the same blood, and used for measurements of platelet adhesion during flow over a collagen surface, as we previously described (42). Fluo-4-labelled cells flowing across the field of view were counted, and the percentage of these cells that adhere stably (adherent for $>20 \mathrm{~s}$ ) was estimated. At a shear rate of $1000 \mathrm{~s}^{-1}, 81.7 \pm 3.6 \%$ of wild type and $78.1 \pm 3.0 \%$ of Prkca ${ }^{-1}$ platelets became stably adherent (mean $\pm S E M, n=5 ; p>0.05$ ), indicating no significant difference in initial stable adhesion to collagen. Also, in order to confirm that in the absence of PKC $\alpha$ the 3-dimensional build up of thrombus formation was markedly impaired, reconstructed images were generated from confocal Z-stacks (as described in Methods) and platelet aggregate (thrombus) volumes estimated. The mean volume of representative platelet aggregates on the coverslips after flow at $1000 \mathrm{~s}^{-1}$ was $570 \pm 168 \mu \mathrm{m}^{3}$ and $72.3 \pm 7.4$ $\mu \mathrm{m}^{3}$ for wild type and Prkcal- blood, respectively (mean $\pm \mathrm{SEM}, \mathrm{n}=5 ; \mathrm{p}<0.05$ ). The major secretory defect seen in Prkcal- platelets was likely to be responsible for the impairment of thrombus formation, since addition of $\operatorname{ADP}(20 \mu \mathrm{M})$ to the blood in the flow chamber was capable of rescuing thrombus formation (Figs 6A (lower panels) \& 6B). This indicated that the thrombotic process under flow conditions markedly relies upon the contribution to secretion made by PKC $\alpha$.

A

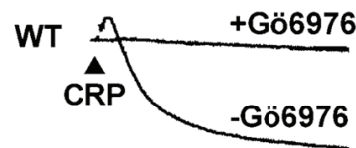

B

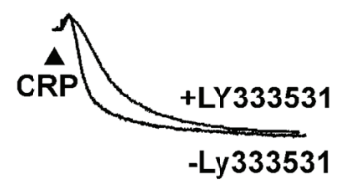

C
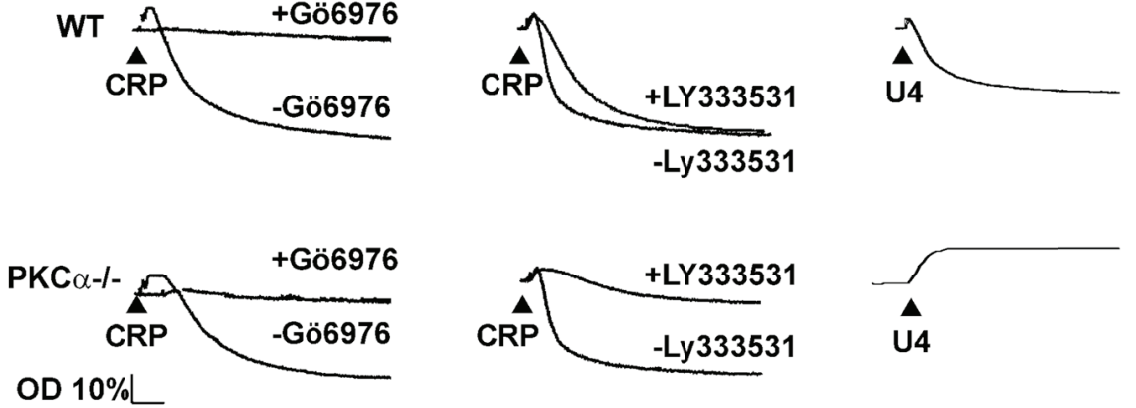

$1 \mathrm{~min}$

Figure 5. Combined pharmacological and genetic approaches reveal redundancy between PKC $\alpha$ and PKC $\beta$ for regulation of platelet aggregation. (A) \& (B) Washed platelets from wild type (WT) or $\mathrm{Prkca}^{-/}$mice were pre-treated (10 min) either with the classical PKC isoform inhibitor Gö6976 (1 $\left.\mu \mathrm{M}\right)$ $(A)$, the PKC $\beta$-selective inhibitor LY333531 (10 $\mu \mathrm{M})(B)$, or with DMSO as vehicle control and stimulated with collagen-related peptide (CRP, $5 \mu \mathrm{g} / \mathrm{ml}$ ) and aggregation was monitored by turbidimetric aggregometry. Traces shown are representative of 5 independent experiments. (C) Washed platelets from wild type (WT) or Prkca ${ }^{-1}$ mice were stimulated with $U_{46619}(10 \mu \mathrm{M})$ and aggregation assessed turbidimetrically. Traces shown are representative of 4 independent experiments.

It was important finally to assess the role in vivo of $\mathrm{PKC} \alpha$ in regulating thrombus formation. Figure 7 shows data where we apply a laser-induced endothelial injury model in 
cremaster muscle arterioles, described previously $(43,44)$. A marked difference is evident between wild type controls and mice lacking PKC $($ Fig. 7), since in the absence of PKC $\alpha$ thrombus formation is delayed and with fewer accumulated platelets (see Supplementary Videos 1 \& 2). Importantly however the ability of Prkca $^{-1-}$ to undergo normal hemostasis was demonstrated by the lack of alteration in tail bleed time (Fig. $7 \mathrm{E}$ ) and by the lack of any fecal occult blood (data not shown). This may indicate that compensatory mechanisms are sufficient to allow normal haemostasis, but are not sufficient to support normal thrombus formation, and may reflect the observation that Prkca $^{-/-}$platelets adhere normally to collagen under static and flow conditions (Figs $1 \& 6$ ), but have a marked reduction in subsequent platelet-platelet interaction (Fig. 6).

A
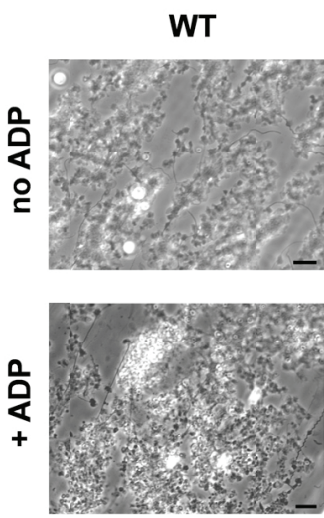

PKC $\alpha^{-1-}$
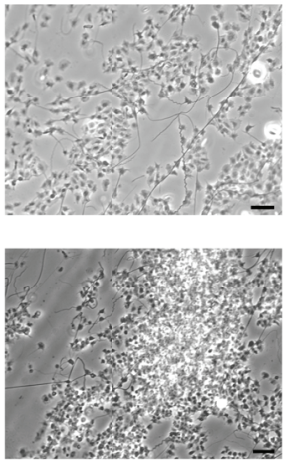

B
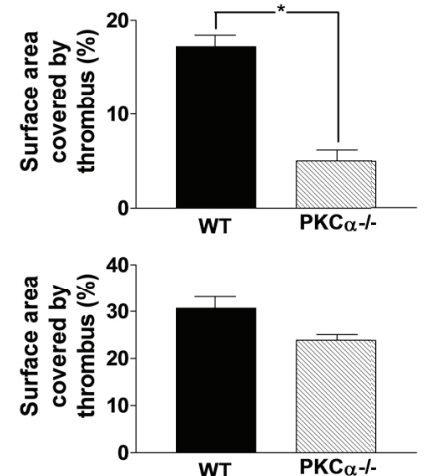

Figure 6. PKC $\alpha$ regulates thrombus formation in vitro. Heparin/PPACK-anticoagulated blood from WT or Prkca $^{-1-}$ mice was flowed over collagen (shear rate $1000 \mathrm{~s}^{-1}$ ). For lower panels of $(A)$ and $(B), A D P$ solution was co-infused at a $10 \%$ flow rate $(20 \mu \mathrm{M}$ ADP, final concentration). (A) shows representative phase-contrast images after $4 \mathrm{~min}$. Images were taken under $63 x$ oil immersion and the bars represent $20 \mu \mathrm{m}$. (B) shows surface area coverage with thrombi (mean $\pm S E M, n \geq 3, * p<0.0001$ ).

\section{Discussion}

Platelets play a central role in mediating atherothrombosis and are therefore the target of numerous therapies aimed at reducing platelet activity, particularly in the prevention of coronary artery thrombosis in heart attacks (45). Protein kinase $C$ is established, largely by pharmacological studies, as a major regulator of multiple platelet activities (2), and it is increasingly clear that the different isozymes of PKC expressed in platelets perform distinct functions. There is a difficulty of interpretation of data from some pharmacological studies however because of the lack of selectivity of the reagents available to target specific PKC isozymes. Here we used a genetic approach to demonstrate definitively, for the first time, the role played by PKC $\alpha$ in regulating platelet function and thrombus formation. Importantly the study revealed a key role for PKC $\alpha$ in regulating granule biogenesis and exocytosis, which was essential for thrombus formation, since ablation of thrombus formation in Prkca $^{-/-}$platelets could be rescued by addition of exogenous ADP. The findings reveal PKC $\alpha$ to be a potential drug target for antithrombotic therapy, since selective inhibitors would 
exert a major effect upon thrombus formation whilst sparing primary platelet adhesive functions.

A

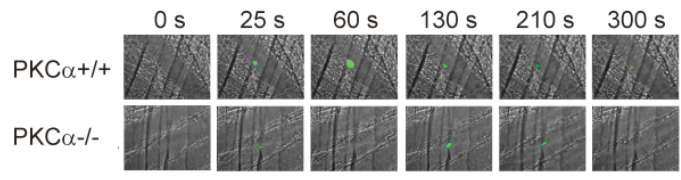

C

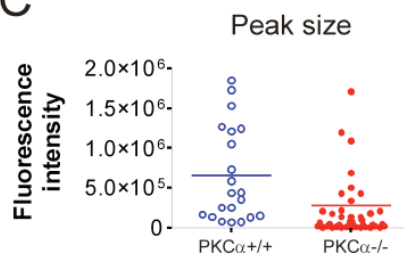

$\mathrm{D}$

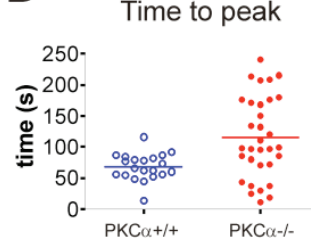

B
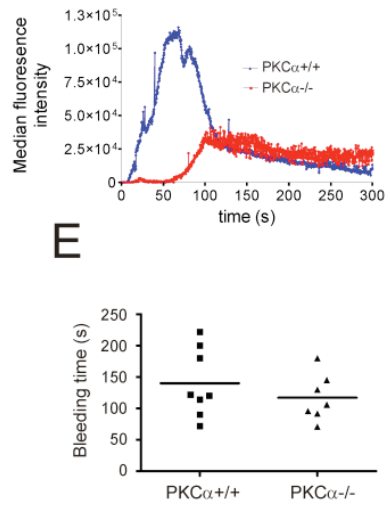

Figure 7. In vivo thrombus formation is impaired in the absence of $P K C \alpha$, but tail bleeding time is normal. (A)-(D) Mice were either Prkcal- or littermate-matched wild-type controls. Platelets were labelled in vivo with Alexa 488, as described in Methods. (A) Platelets (green) composited with brightfield images (black/white) of the cremaster arteriole were viewed at $\times 40$ magnification and images were acquired using a digital CCD camera (SensiCam II, Cooke Corporation) with a $640 \times 480$ pixel array. (B) Traces shown are median integrated platelet fluorescence of 15 thrombi induced in 3 or more mice for each group. Fluorescent intensity of platelets in arbitrary units is presented as a function of time. (C) Data are presented as a scatter diagram, and the horizontal bar is mean of 15 thrombi induced in at least 3 mice for each group. There is a significant reduction in thrombus intensity in $\mathrm{Prkca}^{-1-}$ by comparison with wild type controls (mean Prkca ${ }^{+/+}, 652200$; mean Prkcal, $279600 ; \mathrm{p}<0.05$ ). (D) Time to reach peak thrombus size also significantly differed from wild type controls, with mean data shown by the horizontal bars (mean Prkca ${ }^{+/+}, 68.1 \mathrm{~s} ;$ mean Prkca $\left.{ }^{-1}, 115.2 \mathrm{~s} ; \mathrm{p}<0.01\right)$. (E) Mice were anesthetized and a transverse incision made with a scalpel at a position where the diameter of the tail was 2.25 to $2.5 \mathrm{~mm}$. The tail was immersed in normal saline $\left(37^{\circ} \mathrm{C}\right)$ in a hand-held test tube. The time from incision to cessation of bleeding was recorded, and mean times are shown as horizontal bars. No significant difference was seen comparing wild type mice (mean $140 \mathrm{~s}$ ) with $\mathrm{Prkca}^{-1-}$ (mean $117 \mathrm{~s} ; \mathrm{p}>0.05$ ). For color version, see page 187 .

It has recently been shown pharmacologically that PKC isoforms exert dual control of thrombus formation by mediating secretion and integrin activation under flow, whilst suppressing phosphatidylserine exposure and subsequent thrombin generation and coagulation (7). There is incomplete and largely indirect evidence that, of the various isoforms of PKC expressed in platelets, PKC $\alpha$ plays a role in regulation of integrin $\alpha$ llb $\beta_{3}$ activation (36). The study by Han et al. (33) elegantly showed that PKC $\alpha$ was required for integrin $\alpha$ llb $\beta_{3}$ activation in a reconstituted cell system rather than in the platelet. The study by Tabuchi et al. (18) used an in vitro permeabilised platelet system to investigate receptor-independent calcium-mediated activation of platelet aggregation. Although this latter study demonstrated that added purified PKC $\alpha$ was able to support a calcium-induced platelet aggregation response, the approach has limitations such that receptor-mediated activation is lost. 
Nonetheless, these studies had provided indications that PKC $\alpha$ may regulate platelet integrin activation and aggregation, and it was therefore now essential to perform a geneticbased definitive study of the role played by PKC $\alpha$ in mediating these events.

The marked knockdown in activation of integrin $\alpha \mathrm{llb} \beta_{3}$ in response either to thrombin or CRP in platelets lacking $\mathrm{PKC} \alpha$, paralleled a deficit in the ability of these platelets to undergo aggregation at submaximal concentrations of agonist. This was consistent with the impaired ability of Prkca $^{-/}$platelets to form a thrombus, in blood flowing over a collagencoated surface. The effect on aggregation could however be overcome by increasing the concentration of agonist, and the reasons for this may derive from several factors. First, it is known that integrin $\alpha$ llb $\beta_{3}$ activation in response to strong agonists is in part regulated independently of PKC $(3,46)$. Further, studies with integrin blockers have shown that not all $\alpha \mathrm{llb} \beta_{3}$ receptors are required to be activated (e.g. by the PKC-independent pathway) for a full platelet aggregation response in the platelet aggregometer (47). Thus it may be possible to achieve a maximal aggregation response with a markedly reduced expression of activated integrin. In contrast, in thrombus formation in flowing whole blood, stable platelet aggregation critically depends on limited levels of autocrine produced ADP, and hence limited and reversible integrin activation (48). Under these more physiological conditions, the number of activated integrin receptors may be low, and therefore the marked reduction in integrin activation seen in Prkca ${ }^{-1-}$ platelets may make a significant reduction in the ability of platelets to form thrombi. Additionally we provided evidence for redundancy between $P K C \alpha$ and $P K C \beta$ in Fig. 5, where selective inhibition of $P K C \beta$ only significantly attenuated aggregation responses in Prkcal- platelets, not wild type platelets, indicating redundant functions of these two isoforms. It is also significant to note that, in assays for occult blood in feces, no gastrointestinal bleeding was detected in either wild type $(n=5)$ or $\operatorname{Prkca}^{-/-}(n=6)$ mice. Together with the data shown in Fig. 7, where no difference in tail bleeding time is seen in $\mathrm{PrkCa}^{-1-}$ compared to wild type controls, this also implies redundancy of signaling molecules for regulation of hemostasis in vivo.

There is significant contribution to the effects of $\mathrm{PKC} \alpha$ ablation on aggregation and thrombus formation by regulation of granule secretion, since these processes greatly rely upon autocrine ADP release (49). Clearly both dense and $\alpha$-granule secretion are markedly disrupted in platelets lacking $\mathrm{PKC} \alpha$, and evidence that this is functionally critical is provided by the rescue experiments, shown in Fig. 4 for aggregation and Fig. 6 for in vitro thrombus formation, where addition of exogenous ADP recovers the deficits in these responses seen in Prkca $^{-/}$platelets.

The role of unspecified PKC isoforms as a family in the regulation of secretion had been shown pharmacologically by several groups (3-6). The role of the PKC $\alpha$ isoform in agonist-independent secretion has been demonstrated previously in an artificial permeabilised platelet system (17). Although valuable to indicate the role of $P K C \alpha$, it was now important to address the issue by a definitive genetic approach. Indeed the only study to date to address specific PKC isoform function in secretion in platelets, in a combined genetic and pharmacological approach, had shown a major discrepancy in results obtained by the two approaches for PKC $\delta$. Pula et al. (15) had shown that genetic ablation of PKC $\delta$ had no signif- 
icant effect upon dense granule secretion whereas in that study, and previous ones $(9,16)$, the PKC $\delta$-selective inhibitor rottlerin had been shown to enhance dense granule secretion in response to collagen and alboaggregin, operating through the GPVI receptor. The present results provide direct evidence that $\mathrm{PKC} \alpha$ is a major regulator of secretion of $\alpha$-granules, since P-selectin expression is reduced almost to basal levels in $\mathrm{Prkca}^{-{ }_{-}}$platelets in response either to CRP or thrombin. Importantly we show that $\alpha$-granule numbers are equivalent between $\mathrm{PrkCO}^{-/-}$and wild type platelets, and therefore the secretion defect for this granule type reflects a genuine deficiency in the secretory pathway. For dense granules, this study shows an additional knock-down in numbers of granules in $\mathrm{Prkca}^{-1-}$ platelets. This suggests a role for PKC $\alpha$ in biogenesis of dense granules, and is therefore an area that requires further analysis. A number of different elements of the secretory machinery, including SNAP-23, SNAP-25, Munc18a and b, syntaxin 2 and Rab6, have been reported to be PKC substrates (40,50-52), and thereby their phosphorylation may influence secretion. In this study then, phosphorylation of SNAP-23 serves as a potential marker for these events, and may contribute functionally to the process of secretion. However, although SNAP-23 phosphorylation on Serg5 is markedly reduced in $\mathrm{PrkCa}^{-/}$platelets, and this may play a role in regulating $\alpha$-granule secretion, its role in dense granule secretion cannot be clarified from this study.

In summary, we have shown PKC $\alpha$ to play major roles in regulating platelet secretion of $\alpha$-granules, biogenesis and secretion of dense granules, regulation of integrin $\alpha \mathrm{llb}_{3}$ and thrombus formation in vitro and in vivo. $\mathrm{PKC} \alpha$ also regulates platelet aggregation, although the defect in $\mathrm{PrkCa}^{-1-}$ platelets is relative since high concentrations of agonists overcome the deficit, and there is evident redundancy of the action of PKC $\alpha$ with the other expressed classical PKC isoform in platelets, PKC $\beta$. PKC $\alpha$ plays no significant role in regulating adhesion of platelets to collagen-coated surfaces under static or flow conditions, and does not regulate platelet spreading response or outside-in signaling through integrin $\alpha$ llb $\beta_{3}$. It could be argued that because PKC $\alpha$ is the most highly expressed of the PKC isoforms in platelets, its role in platelets may be wide-ranging and effectively mask that of other PKC isoforms that are expressed to lower levels. The evidence presented here however suggests highly specific roles for $\mathrm{PKC} \alpha$, since for instance its absence has no significant effect upon platelet adhesion and spreading on collagen, but markedly suppresses thrombosis. Additionally, if it were so predominant functionally in platelets, knockout of the other PKC isoforms would be predicted not to have functional effects. This is clearly not the case since we have shown absence of PKC $\delta$ to enhance platelet responses to collagen through enhanced filopodia formation (15), and we have more recently shown absence of PKC $\theta$ to enhance $\alpha$-granule secretion and integrin $\alpha$ llb $\beta_{3}$ activation in response to GPVI agonists (53). The group of Shattil have also shown absence of PKCs $\beta$ and $\theta$ to ablate outside-in signaling through integrin $\alpha$ llb $\beta_{3}(37,38)$. These data therefore demonstrate specific functional roles for the different PKC isoforms, including for PKC $\alpha$ in regulating secretion in particular, such that redundancy of activity is not apparent for specific functions.

Genome-wide association analyses of major human diseases have been conducted recently, based upon technical advances in high throughput microarray analyses of SNPs. These studies are introducing major new leads in genes that may be related to disease, and 
although PKC $\alpha$ is not in the top ranking of genes associating with coronary artery disease, a cluster of SNPs in this gene with a maximal p value just over $10^{-3}$ (SNP rs12600582, intronic, minor allele frequency of 0.233 in Europeans) $(34,35)$ may indicate some significance in this disease of polygenic cause. For these reasons PKC $\alpha$ may represent a drug target for antithrombotic therapy, with inhibitors exerting an effect upon thrombus formation but sparing primary platelet adhesive functions. PKC $\alpha$ is already a target for the drug aprinocarsen, an antisense oligonucleotide therapy used in the treatment of specific neoplastic conditions $(54,55)$, and it will now be important to assess whether this or other small molecule-based approaches may represent opportunities in the development of platelet-based antithrombotic drugs in the management of coronary artery disease and other arterial thrombotic diseases.

\section{Acknowledgements}

We thank Elizabeth Aitken for expert technical assistance supporting this work. The authors would like to thank AstraZeneca for the kind gift of AR-C69931MX. SNAP23 (P-T95) antibody was a generous gift from $\mathrm{Dr}$ Paul Roche (NIH, Bethesda). We thank Dr Mark Jepson and Alan Leard for their assistance within the School of Medical Sciences Cell Imaging Facility. We are also grateful to Gini Tilly and Deborah Carter for their assistance with electron microscopy. We thank Professor lan Day and Dr Tom Gaunt (University of Bristol) for valuable discussions and advice regarding genome-wide-association analyses. We thank Majd Protty, University of Birmingham UK for help with analysis of in vivo thrombosis data. The work was supported grants from the British Heart Foundation to AWP (grant nos. RG/05/015, FS/04/023 \& FS/05/017), and from the NIH to SWW (NIHLB HL56652). AWP is a BBSRC Research Development Fellow.

\section{References}

$1 \quad$ Parker PJ \& Murray-Rust J. PKC at a glance. J Cell Sci 117, 131-2 (2004).

2 Harper MT \& Poole AW. Isoform-specific functions of protein kinase C: the platelet paradigm. Biochem Soc Trans 35, 1005-8 (2007).

3 Shattil SJ \& Brass LF. Induction of the fibrinogen receptor on human platelets by intracellular mediators. J Biol Chem 262, 992-1000 (1987).

$4 \quad$ Walker TR \& Watson SP. Synergy between $\mathrm{Ca}^{2+}$ and protein kinase $\mathrm{C}$ is the major factor in determining the level of secretion from human platelets. Biochem J 289 ( Pt 1), 277-82 (1993).

5 Toullec D, Pianetti $P$, Coste $H$, Bellevergue $P$, Grand-Perret $T$, Ajakane M, Baudet V, Boissin P,

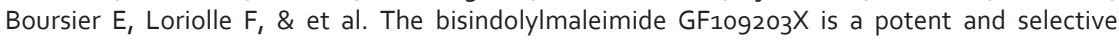
inhibitor of protein kinase C. J Biol Chem 266, 15771-81 (1991).

6 Shattil SJ, Cunningham M, Wiedmer T, Zhao J, Sims PJ, \& Brass LF. Regulation of glycoprotein IIb/IIla receptor function studied with platelets permeabilized by the pore-forming complement proteins C5b-9. J Biol Chem 267, 18424-31 (1992).

7 Strehl A, Munnix IC, Kuijpers MJ, van der Meijden PE, Cosemans JM, Feijge MA, Nieswandt B, \& Heemskerk JW. Dual role of platelet protein kinase $C$ in thrombus formation: stimulation of proaggregatory and suppression of procoagulant activity in platelets. J Biol Chem 282, 7046-55 (2007).

8 Crosby D \& Poole AW. Interaction of Bruton's tyrosine kinase and protein kinase C $\theta$ in platelets. Cross-talk between tyrosine and serine/threonine kinases. J Biol Chem 277, 9958-65 (2002).

9 Murugappan S, Tuluc F, Dorsam RT, Shankar H, \& Kunapuli SP. Differential role of protein kinase $C \delta$ isoform in agonist-induced dense granule secretion in human platelets. J Biol Chem 279, 23607 (2004). 

functional characterization of protein kinase $\mathrm{C}$ isozymes in platelets and HEL cells. J Biol Chem 267, 10011-7 (1992).

11 Crabos M, Imber R, Woodtli T, Fabbro D, \& Erne P. Different translocation of three distinct PKC isoforms with tumor-promoting phorbol ester in human platelets. Biochem Biophys Res Commun $178,878-83$ (1991).

12 Baldassare JJ, Henderson PA, Burns D, Loomis C, \& Fisher GJ. Translocation of protein kinase C isozymes in thrombin-stimulated human platelets. Correlation with 1,2-diacylglycerol levels. J Biol Chem 267, 15585-90 (1992).

13 Wang F, Naik UP, Ehrlich YH, Freyberg Z, Osada S, Ohno S, Kuroki T, Suzuki K, \& Kornecki E. A new protein kinase $\mathrm{C}, \mathrm{nPKC \eta}$, and $\mathrm{nPKC} \theta$ are expressed in human platelets: involvement of $\mathrm{nPKC} \eta$ and nPKC $\theta$ in signal transduction stimulated by PAF. Biochem Biophys Res Commun 191, 240-6 (1993).

14 Khan WA, Blobe G, Halpern A, Taylor W, Wetsel WC, Burns D, Loomis C, \& Hannun YA. Selective regulation of protein kinase $C$ isoenzymes by oleic acid in human platelets. J Biol Chem 268, 5063-8 (1993).

15 Pula G, Schuh K, Nakayama K, Nakayama KI, Walter U, \& Poole AW. PKC $\delta$ regulates collageninduced platelet aggregation through inhibition of VASP-mediated filopodia formation. Blood 108, 4035-44 (2006).

16 Crosby D \& Poole AW. Physical and functional interaction between protein kinase $C \delta$ and Fyn tyrosine kinase in human platelets. J Biol Chem 278, 24533-41 (2003).

17 Yoshioka A, Shirakawa R, Nishioka H, Tabuchi A, Higashi T, Ozaki H, Yamamoto A, Kita T, \& Horiuchi $\mathrm{H}$. Identification of protein kinase $\mathrm{C} \alpha$ as an essential, but not sufficient, cytosolic factor for $\mathrm{Ca}^{2+}$-induced alpha- and dense-core granule secretion in platelets. J Biol Chem 276, 39379-85 (2001).

18 Tabuchi A, Yoshioka A, Higashi T, Shirakawa R, Nishioka H, Kita T, \& Horiuchi H. Direct demonstration of involvement of protein kinase $\mathrm{C} \alpha$ in the $\mathrm{Ca}^{2+}$-induced platelet aggregation. J Biol Chem 278, 26374-9 (2003).

19 Pula G, Crosby D, Baker J, \& Poole AW. Functional interaction of protein kinase C $\alpha$ with the tyrosine kinases Syk and Src in human platelets. J Biol Chem 280, 7194-205 (2005).

20 Dempsey EC, Newton AC, Mochly-Rosen D, Fields AP, Reyland ME, Insel PA, \& Messing RO. Protein kinase $C$ isozymes and the regulation of diverse cell responses. Am J Physiol Lung Cell Mol Physiol 279, L429-38 (2000).

21 Oster $\mathrm{H}$ \& Leitges $\mathrm{M}$. Protein kinase $\mathrm{C} \alpha$ but not $\mathrm{PKC} \zeta$ suppresses intestinal tumor formation in ApcMin/+ mice. Cancer Res 66, 6955-63 (2006).

22 Hara T, Saito Y, Hirai T, Nakamura K, Nakao K, Katsuki M, \& Chida K. Deficiency of protein kinase $\mathrm{C} \alpha$ in mice results in impairment of epidermal hyperplasia and enhancement of tumor formation in two-stage skin carcinogenesis. Cancer Res 65, 7356-62 (2005).

23 Michie AM \& Nakagawa R. The link between PKC $\alpha$ regulation and cellular transformation. Immunol Lett 96, 155-62 (2005).

24 Lahn M, Kohler G, Sundell K, Su C, Li S, Paterson BM, \& Bumol TF. Protein kinase Ca expression in breast and ovarian cancer. Oncology 67, 1-10 (2004).

25 Masur K, Lang K, Niggemann B, Zanker KS, \& Entschladen F. High PKC $\alpha$ and low E-cadherin expression contribute to high migratory activity of colon carcinoma cells. Mol Biol Cell 12, 1973-82 (2001).

26 Morse-Gaudio M, Connolly JM, \& Rose DP. Protein kinase C and its isoforms in human breast cancer cells: relationship to the invasive phenotype. Int J Oncol 12, 1349-54 (1998).

27 Ways DK, Kukoly CA, deVente J, Hooker JL, Bryant WO, Posekany KJ, Fletcher DJ, Cook PP, \& Parker PJ. MCF-7 breast cancer cells transfected with protein kinase $C \alpha$ exhibit altered expression of other protein kinase $\mathrm{C}$ isoforms and display a more aggressive neoplastic phenotype. J Clin Invest 95, 1906-15 (1995).

28 Sliva D. Signaling pathways responsible for cancer cell invasion as targets for cancer therapy. Curr Cancer Drug Targets 4, 327-36 (2004).

29 Pfeifhofer C, Gruber T, Letschka T, Thuille N, Lutz-Nicoladoni C, Hermann-Kleiter N, Braun U, Leitges M, \& Baier G. Defective IgG2a/2b class switching in $P K C \alpha^{-1-}$ mice. J Immunol 176, 6004-11 (2006). 
30 von Essen $M$, Nielsen MW, Bonefeld CM, Boding L, Larsen JM, Leitges M, Baier G, Odum N, \& Geisler C. Protein kinase $\mathrm{C} \alpha$ and PKC $\theta$ are the major PKC isotypes involved in TCR downregulation. J Immunol 176, 7502-10 (2006).

31 Leitges M, Plomann M, Standaert ML, Bandyopadhyay G, Sajan MP, Kanoh Y, \& Farese RV. Knockout of $\mathrm{PKC} \alpha$ enhances insulin signaling through $\mathrm{Pl} 3 \mathrm{~K}$. Mol Endocrinol 16, 847-58 (2002).

32 Braz JC, Gregory K, Pathak A, Zhao W, Sahin B, Klevitsky R, Kimball TF, Lorenz JN, Nairn AC, Liggett SB, Bodi I, Wang S, Schwartz A, Lakatta EG, DePaoli-Roach AA, Robbins J, Hewett TE, Bibb JA, Westfall MV, Kranias EG, \& Molkentin JD. PKC $\alpha$ regulates cardiac contractility and propensity toward heart failure. Nat Med 10, 248-54 (2004).

33 Han J, Lim CJ, Watanabe N, Soriani A, Ratnikov B, Calderwood DA, Puzon-McLaughlin W, Lafuente EM, Boussiotis VA, Shattil SJ, \& Ginsberg MH. Reconstructing and deconstructing agonist-induced activation of integrin $\alpha$ llb $\beta_{3}$. Curr Biol 16, 1796-806 (2006).

34 Samani NJ, Erdmann J, Hall AS, Hengstenberg C, Mangino M, Mayer B, Dixon RJ, Meitinger T, Braund P, Wichmann HE, Barrett JH, Konig IR, Stevens SE, Szymczak S, Tregouet DA, Iles MM, Pahlke F, Pollard H, Lieb W, Cambien F, Fischer M, Ouwehand W, Blankenberg S, Balmforth AJ, Baessler A, Ball SG, Strom TM, Braenne I, Gieger C, Deloukas P, Tobin MD, Ziegler A, Thompson JR, \& Schunkert H. Genomewide association analysis of coronary artery disease. N Engl J Med 357, 443-53 (2007).

35 Consortium TWTCC. Genome-wide association study of 14,000 cases of seven common diseases and 3,000 shared controls. Nature 447, 661-78 (2007).

36 Shattil SJ \& Newman PJ. Integrins: dynamic scaffolds for adhesion and signaling in platelets. Blood 104, 1606-15 (2004).

37 Soriani A, Moran B, de Virgilio M, Kawakami T, Altman A, Lowell C, Eto K, \& Shattil SJ. A role for $\mathrm{PKC} \theta$ in outside-in $\alpha$ llb $\beta_{3}$ signaling. J Thromb Haemost 4, 648-55 (2006).

38 Buensuceso CS, Obergfell A, Soriani A, Eto K, Kiosses WB, Arias-Salgado EG, Kawakami T, \& Shattil SJ. Regulation of outside-in signaling in platelets by integrin-associated protein kinase $C \beta$. J Biol Chem 280, 644-53 (2005).

39 Polgar J, Lane WS, Chung SH, Houng AK, \& Reed GL. Phosphorylation of SNAP-23 in activated human platelets. J Biol Chem 278, 44369-76 (2003).

40 Hepp R, Puri N, Hohenstein AC, Crawford GL, Whiteheart SW, \& Roche PA. Phosphorylation of SNAP-23 regulates exocytosis from mast cells. J Biol Chem 280, 6610-20 (2005).

41 Quinton TM, Kim S, Dangelmaier C, Dorsam RT, Jin J, Daniel JL, \& Kunapuli SP. Protein kinase Cand calcium-regulated pathways independently synergize with $\mathrm{Gi}$ pathways in agonist-induced fibrinogen receptor activation. Biochem J 368, 535-43 (2002).

42 Auger JM, Kuijpers MJ, Senis YA, Watson SP, \& Heemskerk JW. Adhesion of human and mouse platelets to collagen under shear: a unifying model. FASEB J 19, 825-7 (2005).

43 Falati S, Gross P, Merrill-Skoloff G, Furie BC, \& Furie B. Real-time in vivo imaging of platelets, tissue factor and fibrin during arterial thrombus formation in the mouse. Nat Med 8, 1175-81 (2002).

44 Kalia N, Auger JM, Atkinson B, \& Watson SP. Critical role of FcRy-chain, LAT, PLCY2 and thrombin in arteriolar thrombus formation upon mild, laser-induced endothelial injury in vivo. Microcirculation 15, 325-35 (2008).

45 Meadows TA \& Bhatt DL. Clinical aspects of platelet inhibitors and thrombus formation. Circ Res 100, 1261-75 (2007).

46 Crittenden JR, Bergmeier W, Zhang Y, Piffath CL, Liang Y, Wagner DD, Housman DE, \& Graybiel AM. CaIDAG-GEFI integrates signaling for platelet aggregation and thrombus formation. Nat Med 10, 982-6 (2004).

47 Scarborough RM, Kleiman NS, \& Phillips DR. Platelet glycoprotein IIb/IIla antagonists. What are the relevant issues concerning their pharmacology and clinical use? Circulation 100, 437-44 (1999).

48 Cosemans JM, Munnix IC, Wetzker R, Heller R, Jackson SP, \& Heemskerk JW. Continuous signal ing via $\mathrm{Pl} 3 \mathrm{~K}$ isoforms $\beta$ and $\gamma$ is required for platelet ADP receptor function in dynamic thrombus stabilization. Blood 108, 3045-52 (2006).

49 Remijn JA, Wu YP, Jeninga EH, M.J. I, van Willigen G, de Groot PG, Sixma JJ, Nurden AT, \& Nurden $\mathrm{P}$. Role of ADP receptor $\mathrm{P}_{2} \mathrm{Y}_{12}$ in platelet adhesion and thrombus formation in flowing blood. Arterioscler Thromb Vasc Biol 22, 686-91 (2002). 
50 Morgan A, Burgoyne RD, Barclay JW, Craig TJ, Prescott GR, Ciufo LF, Evans GJ, \& Graham ME. Regulation of exocytosis by protein kinase C. Biochem Soc Trans 33, 1341-4 (2005).

51 Fitzgerald ML \& Reed GL. Rab6 is phosphorylated in thrombin-activated platelets by a protein kinase C-dependent mechanism: effects on GTP/GDP binding and cellular distribution. Biochem J 342 ( Pt 2), 353-60 (1999).

52 Reed GL, Houng AK, \& Fitzgerald ML. Human platelets contain SNARE proteins and a Secıp homologue that interacts with syntaxin 4 and is phosphorylated after thrombin activation: implications for platelet secretion. Blood 93, 2617-26 (1999).

53 Hall KJ, Harper MT, Gilio K, Cosemans JM, Heemskerk JW, \& Poole AW. Genetic analysis of the role of protein kinase $C \theta$ in platelet function and thrombus formation. PLoS One 3, e3277 (2008).

54 Hanauske AR, Sundell K, \& Lahn M. The role of protein kinase C $\alpha$ in cancer and its modulation by the novel PKC $\alpha$-specific inhibitor aprinocarsen. Curr Pharm Des 10, 1923-36 (2004).

55 Lahn M, Sundell K, \& Kohler G. The role of protein kinase C $\alpha$ in hematologic malignancies. Acta Haematol 115, 1-8 (2006).

56 Kuijpers MJ, Schulte V, Bergmeier W, Lindhout T, Brakebusch C, Offermanns S, Fassler R, Heemskerk JW, \& Nieswandt B. Complementary roles of glycoprotein VI and $\alpha_{2} \beta_{1}$ integrin in collagen-induced thrombus formation in flowing whole blood ex vivo. FASEB J 17, 685-7 (2003).

57 Munnix IC, Kuijpers MJ, Auger J, Thomassen CM, Panizzi P, van Zandvoort MA, Rosing J, Bock PE, Watson SP, \& Heemskerk JW. Segregation of platelet aggregatory and procoagulant microdomains in thrombus formation: regulation by transient integrin activation. Arterioscler Thromb Vasc Biol 27, 2484-90 (2007). 


\section{Chapter 5}

Functional divergence of platelet protein kinase $C$
isoforms in thrombus formation on collagen

Gilio K, Harper MT, Cosemans JM, Konopatskaya O, Munnix IC, Prinzen L, Leitges M, Liu Q, Molkentin JD, Heemskerk JW, and Poole AW

J Biol Chem. 2010;285:23410-9

Reprinted with permission 


\begin{abstract}
Arterial thrombosis, a major cause of myocardial infarction and stroke, is initiated by activation of blood platelets by subendothelial collagen. The protein kinase C family centrally regulates platelet activation, and it is becoming clear that the individual PKC isoforms play distinct roles, some of which oppose each other. Here, for the first time, we address all four of the major platelet-expressed PKC isoforms, determining their comparative roles in regulating platelet adhesion to collagen and their subsequent activation under physiological flow conditions. Using mouse gene knockout and pharmacological approaches in human platelets we show that collagen-dependent $\alpha$-granule secretion and thrombus formation are mediated by the conventional PKC isoforms, $\mathrm{PKC} \alpha$ and $\mathrm{PKC} \beta$, whereas the novel isoform, $\mathrm{PKC} \theta$, negatively regulates these events. $\mathrm{PKC} \delta$ also negatively regulates thrombus formation but not $\delta$-granule secretion. In addition, we demonstrate for the first time that individual PKC isoforms differentially regulate platelet calcium signaling and exposure of phosphatidylserine under flow. Whereas platelet deficient in PKC $\alpha$ or $\mathrm{PKC} \beta$ showed reduced calcium signaling and phosphatidylserine exposure, these responses were enhanced in the absence of PKCO. In summary therefore, this direct comparison between individual subtypes of PKC, by standardized methodology under flow conditions reveals that the four major PKCs expressed in platelets play distinct non-redundant roles, where conventional PKCs promote and novel PKCs inhibit thrombus formation on collagen.
\end{abstract}

\title{
Introduction
}

Blood vessel damage or rupture of an atherosclerotic plaque exposes subendothelial collagen. Platelets rapidly adhere to collagen and are activated, inducing granule secretion and integrin $\alpha$ llb $\beta_{3}$ activation, and leading to large platelet aggregates. In addition, sustained intracellular calcium signaling induces phosphatidylserine (PS) exposure, which accelerates thrombin generation and coagulation. Together, these processes can result in formation of occlusive thrombi, leading to myocardial infarction or stroke.

The protein kinase $\mathrm{C}$ (PKC) family is comprised of multiple isoforms, which are responsible for a substantial part of the serine/threonine phosphorylation events in many cell types including platelets. The PKC isoforms are grouped into three classes: conventional forms $(\alpha$, $\beta I, \beta I I, \gamma)$ that are activated by $\mathrm{Ca}^{2+} /$ diacylglycerol, novel forms $(\delta, \varepsilon, \eta, \theta)$ activated by diacylglycerol alone, and atypical forms $(\zeta, 1 / \lambda)$, which are diacylglycerol-independent (1). Human and mouse platelets highly express the conventional PKC isoforms $\alpha$ and $\beta$, and the novel isoforms $\delta$ and $\theta(2-6)$, while mouse platelets in addition express $\operatorname{PKC} \varepsilon(7,8)$. Early pharmacological studies, which could not distinguish between different isoforms, showed that key platelet activation processes, such as secretion, integrin $\alpha$ llb $\beta_{3}$ activation and aggregation, are positively regulated by PKC activity (9-13). However, there is also good evidence that PKC has a negative role in platelets, in particular by suppressing $\mathrm{Ca}^{2+}$ signal generation, for example by promoting $\mathrm{Ca}^{2+}$ extrusion $(14,15)$, and desensitizing agonist receptors (16). This raised the intriguing question whether different conventional and novel PKC isoforms may have distinct or even opposing roles in the control of these platelet responses (17). 
There is some evidence to support this hypothesis. In human and mouse platelets, PKC $\alpha$ has been proposed as a key kinase regulating $\alpha$ - and dense granule secretion $(18,19)$ and platelet aggregate formation (20) in response to collagen and other platelet agonists (21). In mouse platelets, PKC $\beta$ positively regulates outside-in $\alpha$ llb $\beta 3$ signaling (22), but not inside-out integrin activation. However, the role of the novel PKC isoforms, PKC $\delta$ and PKC $\theta$, is less straightforward, since both positive and negative signaling functions have been reported $(8,23-25)$.

In light of this lack of a clear role for all PKC isoforms, which may result partly from differences in platelet preparation conditions, anticoagulant and other ex vivo handling of platelet samples by different laboratories, we sought to compare directly platelet responses in physiological flow settings in whole blood. We compared platelet function and thrombotic response to flow over collagen, using blood from mice deficient in each of the four PKC isoforms. The data point to markedly divergent and partly antagonistic roles of the conventional and novel PKC isoforms in collagen-induced platelet activation and thrombus formation. In addition, we show for the first time that individual PKC isoforms differentially regulate platelet calcium signaling and procoagulant activity.

\section{Experimental procedures}

Animals

Animal studies were approved by the local animal care and use committees. All mice were generated as described earlier for PKC $\alpha$ (26), PKC $\beta$ (27), PKC $\delta$ (28) or PKC $\theta$ (29). In all mouse strains, platelet and erythrocyte counts in blood were in the normal range. Wildtype mice were used of the same background and same breeding program as the corresponding knockout mice.

\section{Materials}

H-Phe-Pro-Arg chloromethyl ketone (PPACK) was obtained from Calbiochem, as were Ro318425, Gö6976, PKC $\beta$-inhibitor (3-(1-(3-Imidazol-1-ylpropyl)-1H-indol-3-yl)-4-anilino-1Hpyrrole-2,5-dione) and rottlerin. PKC $\theta$-inhibitor (30) was a kind gift from Boehringer Ingelheim Pharmaceuticals. Annexin A5 labeled with fluorescein isothiocyanate (FITC) was from Nexins Research. Fura-2 and Fluo-4 acetoxymethyl esters, pluronic F-127 and Alexa Fluor (AF)647-labeled annexin A5 came from Molecular Probes. Apyrase (grade V), bovine serum albumin, dichloro fluorescein (DCF) and phorbol myristate acetate (PMA) were from Sigma. Fibrillar type I collagen (Horm) was from Nycomed. Convulxin was purified as described (31). FITC-labeled PAC 1 mAb against activated human $\alpha$ llb $\beta_{3}$ came from BD Biosciences. FITClabeled anti-human-CD62 (P-selectin) mAb from Sanquin. FITC-labeled anti-mouse CD62 mAb was from Emfret Analytics, as was FITC-labeled JON/A mAb. Other materials were obtained from sources indicated before $(32,33)$.

\section{Blood collection and platelet preparation}

Blood was taken from aspirin-free healthy volunteers, who gave full informed consent. Human platelet-rich plasma (PRP) and washed platelets were prepared by centrifugation 
(34). Platelets were resuspended in Hepes buffer pH 7.45 (136 mM NaCl, $10 \mathrm{mM}$ Hepes, 2.7 $\mathrm{mM} \mathrm{KCl}, 2 \mathrm{mM} \mathrm{MgCl} 2,1 \mathrm{mg} / \mathrm{ml}$ glucose, $1 \mathrm{mg} / \mathrm{ml}$ bovine serum albumin, $0.2 \mathrm{U} / \mathrm{ml}$ apyrase) at a concentration of $2 \times 10^{8} / \mathrm{ml}$.

Mouse blood was obtained by cardiac puncture under terminal anesthesia. For flow studies, blood was collected into $40 \mu \mathrm{M}$ PPACK, $5 \mathrm{U} / \mathrm{ml}$ heparin and $40 \mathrm{U} / \mathrm{ml}$ fragmin. For washed platelets, mouse blood was collected into $129 \mathrm{mM}$ citrate, $1 \mathrm{U} / \mathrm{ml}$ heparin and $5 \mathrm{mM}$ glucose (32). Washed cells were suspended in modified Tyrode's Hepes buffer pH 7.45 (134 $\mathrm{mM} \mathrm{NaCl}, 20 \mathrm{mM}$ Hepes, $12 \mathrm{mM} \mathrm{NaHCO} 3,2.9 \mathrm{mM} \mathrm{KCl}, 1 \mathrm{mM} \mathrm{MgCl}$, $0.34 \mathrm{mM} \mathrm{Na} 2 \mathrm{HPO}_{4}, 5$ $\mathrm{mM}$ glucose). Platelets were counted with a Coulter counter and adjusted to the appropriate density.

\section{Thrombus formation}

Thrombus formation on collagen under flow was determined as described (32). Briefly, PPACK-anticoagulated mouse blood was flowed through a transparent, parallel-plate flow chamber ( $50 \mu \mathrm{m}$ deep, $3 \mathrm{~mm}$ wide), sealed with a collagen-coated coverslip, at a shear rate of $1000 \mathrm{~s}^{-1}$. To measure thrombus volumes, platelets in blood were prelabeled with DCF (35). Activated platelets in thrombi were post-stained with FITC-annexin $A_{5}(0.5 \mu \mathrm{g} / \mathrm{ml})$, or with AF647-annexin A5 (1:400) and FITC-labeled anti-P-selectin mAb (1:40). Phase-contrast and fluorescence images were recorded with a DM-IRB fluorescence microscope and an Orca ER camera (Leica) or with a Nikon Diaphot 200 microscope and an EM-CCD camera (Hamamatsu). Confocal images were captured in real-time using a Zeiss/BioRad E6ooFN multiphoton system (36). Phase-contrast and (confocal) fluorescence images were taken from at least 10 randomly chosen microscopic fields. Images were analyzed for area coverage, integrated fluorescence intensity or integrated fluorescence pixel density using ImagePro software (Media Cybernetics), as described before (34). For P-selectin staining, the extent of integrated fluorescence intensity depends on the number of platelets (i.e. the thrombus size) and the fluorescence per platelet. In contrast, integrated pixel density gives a measure of the amount of fluorescence per platelet. In control experiments, treatment of a pre-formed thrombus with thrombin resulted in a further increase (approximately 2.5 -fold) in mean pixel density of P-selectin fluorescence (data not shown). Both integrated fluorescence intensity and pixel density are given in figures 2 and 4 . In contrast, individual platelets strain strongly with annexin $A_{5}$ or remain unstained, since annexin $A_{5}$ polymerizes when binding to PS-exposing surfaces. The pixel density (i.e. staining per platelet) was therefore the same for all mouse strains, but the integrated fluorescence intensity is indicative of the number of PS-exposing platelets. Thrombus volume of DCF-labeled platelets was measured from confocal stacks of fluorescence images (35).

\section{Measurement of single-cell $\mathrm{Ca}^{2+}$ responses under flow}

Washed murine platelets were incubated with $8 \mu \mathrm{M}$ Fluo- 4 acetoxymethyl ester and 0.2 $\mathrm{mg} / \mathrm{ml}$ pluronic $\mathrm{F}-127$ for $45 \mathrm{~min}$ at ambient temperature under gentle rotation (37). Dyeloaded platelets were added to PPACK-anticoagulated blood from the same mouse strain to give $10 \%$ labeled platelets. During the first min of high-shear blood flow, images were 
captured from the collagen surface at $5 \mathrm{~Hz}$. Pseudo-ratio F/Fo values were converted into nanomolar concentrations of $\left[\mathrm{Ca}^{2+}\right]_{\mathrm{i}}$ using predefined calibration parameters (38).

\section{Measurement of single platelet adhesion to collagen}

Fluo-4-loaded platelets were added to whole blood as described above. Even in unactivated Fluo-4-loaded platelets there is basal fluorescence, which can be used to track the platelets under flow conditions, as described previously (37). Stably-adhered platelets were defined as labeled cells that remained in one position on a collagen fiber for $>30$ seconds.

\section{Measurement of PKC activity}

PKC activity was determined by Ser phosphorylation of modified PKC pseudo-substrate RFARKGSLROKNV (39), using a biotinylated mAb recognizing the phosphorylated form (Calbiochem). Washed platelets $\left(1 \times 10^{8} / \mathrm{ml}\right)$, pretreated with indicated inhibitors (10 $\mathrm{min}$ ), were pelleted and immediately sonicated on ice, and then further processed as indicated by the manufacturer.

\section{Platelet aggregation and flow cytometry}

Washed platelets were pretreated for 10 min with $\mathrm{Me} 2 \mathrm{SO}$ vehicle or indicated inhibitor. Platelet aggregation was measured under stirring by light transmission aggregometry at $37^{\circ} \mathrm{C}$. Using flow cytometry, $\alpha$-granule secretion was assessed as P-selectin exposure with FITC-labeled anti-human or anti-mouse CD62 mAb. Similarly, $\alpha$ llb $\beta_{3}$ activation was assessed with FITC-labeled mAb against activation epitopes of this integrin (36).

\section{Calcium responses in suspension}

Fura-2-loaded platelets $\left(1 \times 10^{8} / \mathrm{m}\right)$, prepared as described (33), were used to measure changes in cytosolic $\left[\mathrm{Ca}^{2+}\right]_{i}$ by calibrated ratio fluorimetry. Time integrals of $\left[\mathrm{Ca}^{2+}\right]_{i}$ (in $\mathrm{nM}$ ) were determined as before.

\section{Statistical analysis}

Differences between experimental groups were tested for significance with the nonparametric Mann-Whitney $U$ test, using SPSS. Differences in frequency distributions of features with mean values per field were determined by $X^{2}$ test (34).

\section{Results}

Deficiency in conventional isoforms $P K C \alpha$ or 8 impairs collagen-induced thrombus formation and platelet activation under flow

To investigate the functional consequences of specific PKC isoform deficiency, we used an in vitro flow model of platelet-vessel wall interaction, in which whole blood is perfused over a collagen-coated surface at defined, arterial shear rate $(32,37,40,41)$. Previous human studies demonstrated that general inhibition of PKC almost fully abrogates the formation of platelet aggregates, whereas adhered platelets show increased procoagulant activity, as assessed by labeled annexin $A_{5}$, which detects exposed PS (17). During flow over collagen at 
$1000 \mathrm{~s}^{-1}$, wildtype mouse platelets rapidly assembled into large aggregates and by contrast, after 4 min (Fig. $1 \mathrm{~A})$ or longer perfusion times, $\mathrm{PKC \alpha}^{-1-}$ platelets were markedly impaired in aggregate formation, in agreement with our previous report (18). Formation of pseudopods and lamellipods of the adhered PKC $\alpha^{-1-}$ platelets was however normal. Quantitative analysis showed a reduction in platelet deposition by $70 \%$ (Fig. 1B), while morphometric analysis of the PKC $\alpha^{-1-}$ thrombi showed that mostly single cells or small-sized aggregates were present (Fig. 1C). The latter observation was confirmed by measurement of thrombus volume after perfusion, using platelets prelabeled with DCF. Mean volumes of $323 \pm 76$ and $89 \pm 18 \mu \mathrm{m}^{3}$ for wild type and PKC $\alpha^{-/-}$platelets, respectively, were achieved (mean \pm S.E., $n \geq 34, p<0.01$ ).

A

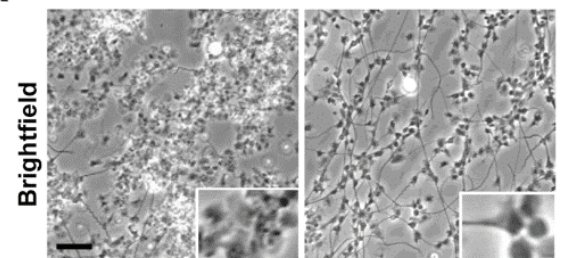

$\mathrm{PKC}^{+/+}$
$\mathrm{PKC} \alpha^{-1-}$

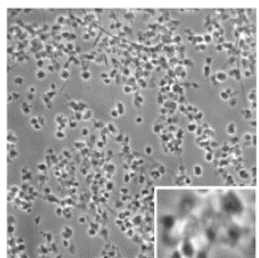

PKC $\beta^{+/+}$

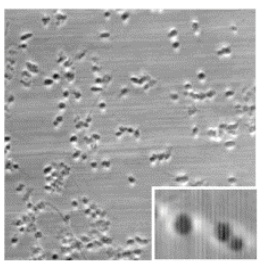

PKC $\beta^{-1-}$

B

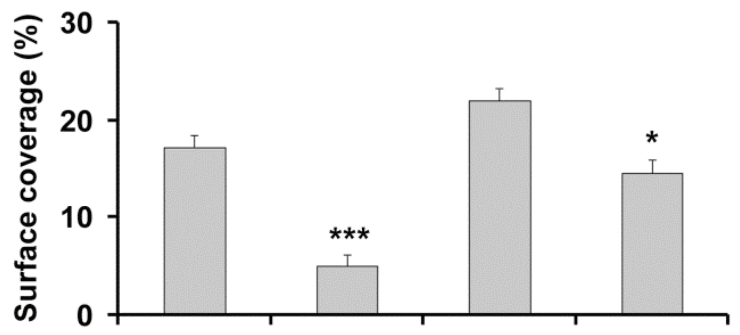

C

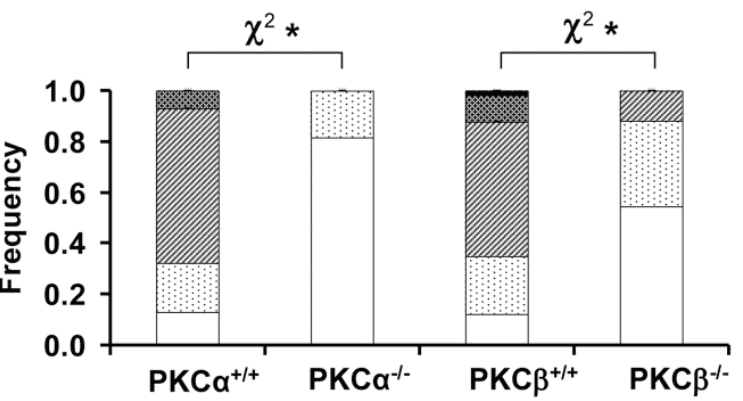

1 to 3 plt 3 to 8 plt $\mathbb{Z} 8$ to 40 plt 40 to 130 plt $>130$ plt

Figure 1. Deficiency of PKC $\alpha$ or $\beta$ suppresses thrombus formation on collagen under flow. Whole blood from $\mathrm{PKC}^{-1}, \mathrm{PKC}^{-1}$ or matched wildtype mice was flowed over a collagen surface at a shear rate of $1000 \mathrm{~s}^{-1}$. A, Representative phase contrast images after 4 min of perfusion (bar $20 \mu \mathrm{m}$, inserts $5^{\times}$ magnified). B, Quantification of platelet deposition on collagen surface. C, Morphometric analysis of aggregates on collagen surface with indicated numbers of platelets per feature. Means \pm S.E.M. $\left(n=3^{-}\right.$ $6), * p<0.05, * * * p<0.001$ vs. corresponding wildtypes; $x^{2}$ test $* p<0.05$. 
Interestingly, deficiency in PKC $\beta$ also resulted in diminished platelet deposition and formation of smaller size aggregates on collagen (Fig. 1), and thrombus volume was reduced to $95 \pm 24 \mu \mathrm{m}^{3}$. Platelet deposition during thrombus formation involves both primary adhesion of platelets to collagen and platelet-platelet aggregation. Analysis of primary platelet adhesion to collagen however showed no effect of loss of either PKC $\alpha$ or $\beta$ since fractions of stably adhered platelets were similar for wild type and $\mathrm{PKC} \alpha^{-/-}$blood $(80 \pm 4 \%$ and $77 \pm 3 \%)$, as well as for wild type and $\mathrm{PKC} \beta^{-1-}$ blood $(80 \pm 5 \%$ and $81 \pm 4 \% ; n=4$ experiments, $p>0.5$ ). Hence, the impaired thrombus formation is not a consequence of defective adhesion to collagen but rather a result of reduced platelet-platelet cohesion.

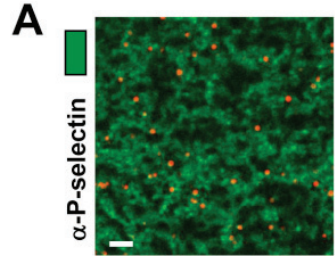

$\mathrm{PKCa}^{+/+}$

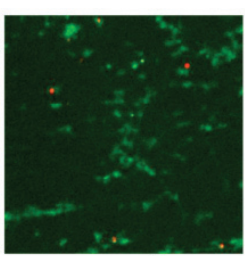

$\mathrm{PKC} \alpha^{-1-}$

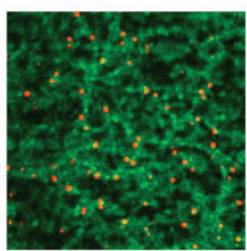

$\mathrm{PKC}^{+/+}$

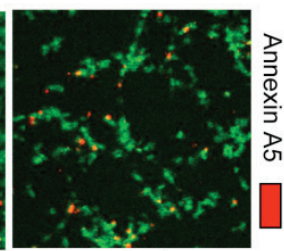

PKC $\beta^{-1-}$

B P-selectin

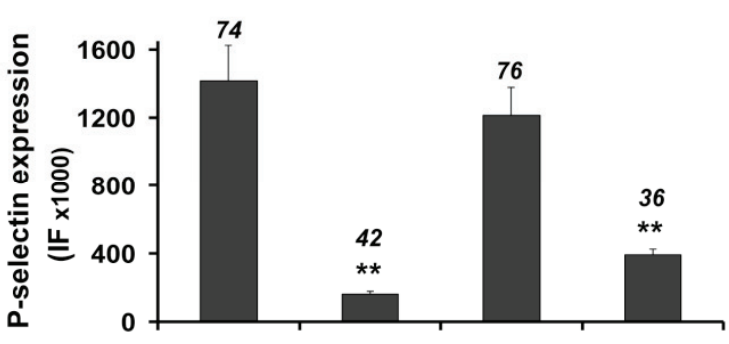

$P S$ exposure

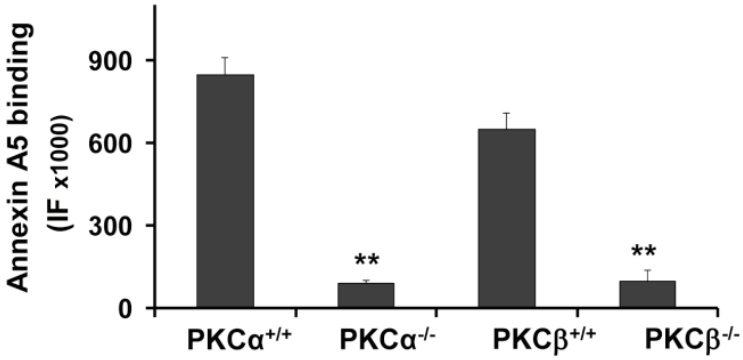

Figure 2. Deficiency of PKC $\alpha$ or $\beta$ suppresses collagen-induced activation of platelets in thrombi. Whole blood from indicated mice was flowed over collagen (see Fig. 1), and platelet thrombi were double-stained with FITC-anti-mouse CD62P mAb (green) and AF647-annexin A5 (red). A, Representative confocal images $(180 \times 180 \mu \mathrm{m})$ after staining. $B$, Integrated fluorescence intensity presenting activated platelets in arbitrary units, to quantify cumulative expression of P-selectin and PS. Means \pm S.E.M. $(n=3-5) ; * * p<0.01$ vs. corresponding wildtypes. Numbers above each bar indicate the mean integrated pixel density of P-selectin staining, an indication of staining per platelet. For color version, see page 188. 
To investigate whether the reduced thrombus formation was accompanied by altered platelet activation, thrombi formed on the collagen surface were labeled for the $\alpha$-granule secretion marker, P-selectin, and for procoagulant activity, with PS-binding annexin A5. Fig. $2 \mathrm{~A}$ shows images obtained after dual staining (staining with each probe individually gave similar results). For both $\mathrm{PKC}^{-{ }^{-}}$and $\mathrm{PKC}^{-/-}$thrombi, measurement of integrated fluorescence intensity demonstrated a dramatic reduction in surface expression of $\mathrm{P}$-selectin and PS, when compared to wildtypes (Fig. 2B). Although this reduction was partly explained by the diminished platelet deposition, the P-selectin mean fluorescence intensity per pixel was also reduced, suggesting a lower degree of $\alpha$-granule secretion per platelet. Interestingly, in contrast to general PKC inhibition studies (17), where greater numbers of platelets show PS exposure, fewer platelets exposed PS in the absence of PKC $\alpha$ or PKC $\beta$ compared to wild type controls (Fig. 2A).

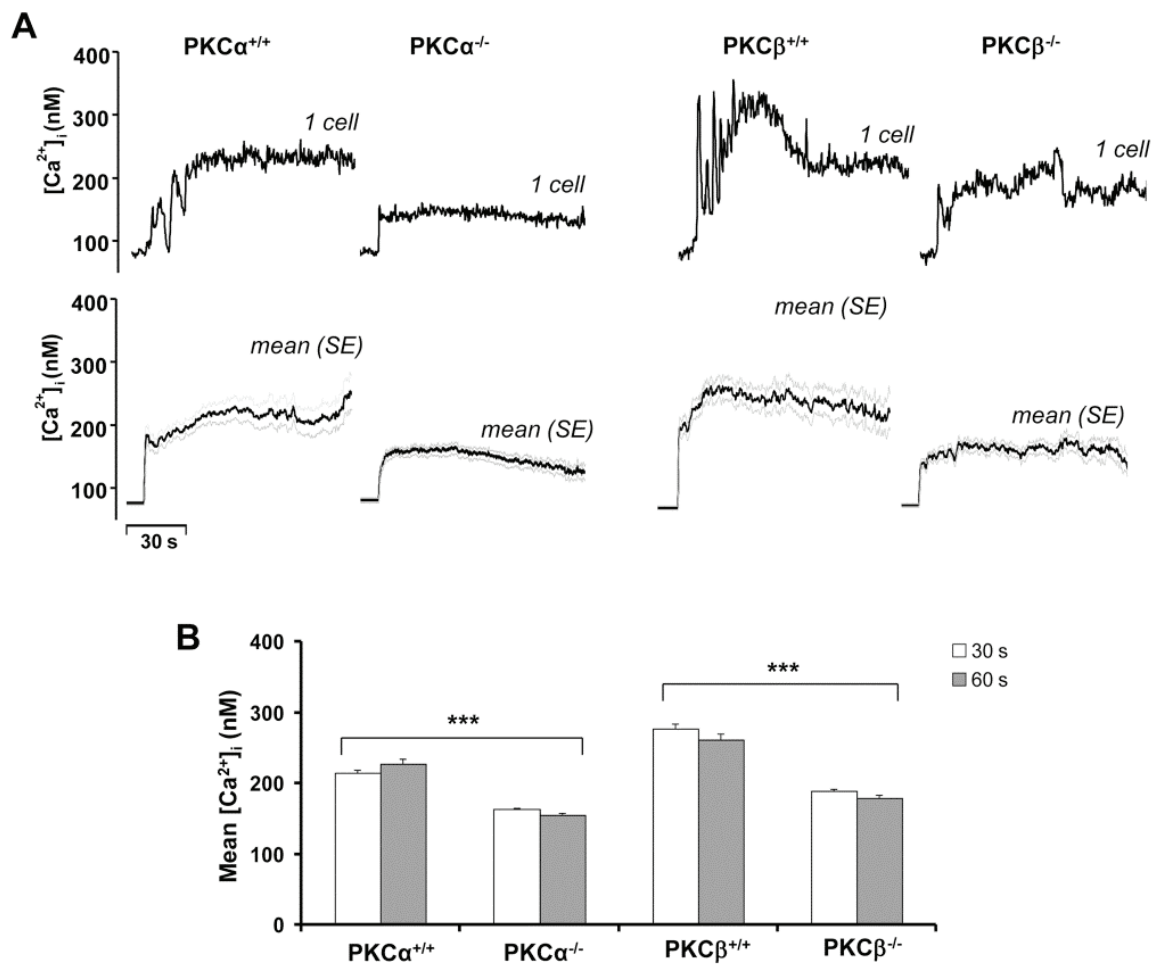

Figure 3. Deficiency of $\mathrm{PKC} \alpha$ or $\beta$ suppresses collagen-induced $\mathrm{Ca}^{2+}$ signaling under flow, but not adhesion. Whole blood containing autologous Fluo-4-loaded platelets was flowed over collagen (see Fig. 1), while fluorescence images of adherent platelets were captured in real-time (5 Hz). A, Traces of pseudo-ratioed changes in $\left[\mathrm{Ca}^{2+}\right]_{i}$ from representative, single platelets as well as mean traces (S.E.) from overlays of $30-40$ platelets. B, Quantitative analysis of increased $\left[\mathrm{Ca}^{2+}\right]_{i}$ at $30 \mathrm{~s}$ (white) and $60 \mathrm{~s}$ (gray) after initial response. No quantitative differences in response between wild type mice were seen. After $30 \mathrm{sec}$, averaged rises in $\left[\mathrm{Ca}^{2+}\right]_{i}$ were $225 \pm 26.3 \mathrm{nM}$ for PKC ${ }^{+/+}$platelets, and $270 \pm 33.9 \mathrm{nM}$ for PKC $\beta^{+/+}$platelets $(n=3)$. Data are means \pm S.E.M. $(n=30-40,3-4 \text { experiments })_{i} * * * p<0.001$ vs. corresponding wildtypes. 
The results so far suggested that deficiency in PKC $\alpha$ or $\beta$ impairs platelet activation by collagen. To investigate this further and mechanistically, we monitored $\mathrm{Ca}^{2+}$ signaling in collagen-adhered platelets during whole-blood perfusion $(36,37)$. Analysis of the $\mathrm{Ca}^{2+}$ responses showed that although platelets from $\mathrm{PKC} \alpha^{-1-}$ and $\mathrm{PKC} \beta^{-1-}$ mice were still capable of prolonged rises in $\left[\mathrm{Ca}^{2+}\right]_{i}$ after adhesion, these rises were lower in amplitude by comparison with those of corresponding wildtype platelets (Fig. 3A\&B).

Together, these results indicate that deficiency in either $\mathrm{PKC} \alpha$ or $\mathrm{PKC} \beta$ does not change primary platelet adhesion to collagen under flow, but results impaired secretion and aggregate formation and in lower GPVI-induced $\mathrm{Ca}^{2+}$ rises and PS exposure. Furthermore, PKC $\alpha$ and $\beta$ isoforms appear to play non-redundant roles.

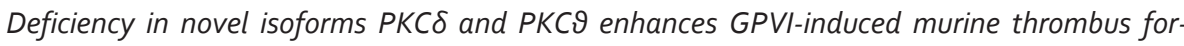
mation and platelet activation under flow

To investigate the contribution of the major novel PKC isoforms in thrombus formation, flow experiments on collagen were carried out with blood from mice lacking PKC $\theta$ or $\delta$. Platelets from $\mathrm{PKC} \theta^{-1-}$ or $\mathrm{PKC} \delta^{-1-}$ mice readily assembled into aggregates (Fig. $4 \mathrm{~A}$ ). In comparison to wild type controls, these thrombi covered more of the collagen surface, and more frequently consisted of larger-size aggregates (Fig. 4B-C). Thrombus volumes from wild type, $\mathrm{PKC} \theta^{--}$, and $\mathrm{PKC} \delta^{--}$thrombi were $490 \pm 136,1923 \pm 456$ and $2304 \pm 261 \mu \mathrm{m}^{3}$, respectively ( $n \geq 16, p<0.05$ ). Thrombi on collagen were then stained for P-selectin and PS (Fig. 5A). The increased deposition of $\mathrm{PKCO}^{-/-}$platelets was accompanied by higher integrated fluorescence intensities of both stains (Fig. ${ }_{5} \mathrm{~B}$ ) and a higher fluorescence per pixel for P-selectin staining, suggesting an overall higher activation state of the platelets. In contrast, this was not observed for $\mathrm{PKC} \delta^{-1-}$ platelets. As for $\mathrm{PKC} \alpha^{--}$and $\mathrm{PKC} \beta^{-1}$, similar fractions of labeled wildtype $(87 \pm 5 \%), \mathrm{PKCO}^{-/-}(80 \pm 2 \%)$ and $\mathrm{PKC}^{-1-}(84 \pm 4 \%)$ platelets were found stably adherent to collagen under flow ( $n=4-5$ experiments, $p>0.2)$, suggesting that enhanced thrombus formation does not result from increased adhesion to collagen, but rather enhanced ability to form large aggregates.

Using Fluo-4-loaded platelets, collagen-adhered $\mathrm{PKC}^{-1-}$ platelets, but not $\mathrm{PKC} \delta^{-1-}$ platelets, exhibited higher amplitude rises in $\left[\mathrm{Ca}^{2+}\right]_{\mathrm{i}}$ in comparison to the corresponding wildtype cells (Fig. 6A). The increased $\mathrm{Ca}^{2+}$ signal in $\mathrm{PKC}^{-1-}$ platelets was detectable at both earlier (30 s) and later (6o s) time points (Fig. 6B). Together, these results suggest that absence of PKC $\theta$ augments collagen-dependent thrombus formation under flow by increasing GPVI-induced $\mathrm{Ca}^{2+}$ signaling, and downstream responses such as $\alpha$-granule secretion and procoagulant activity. 


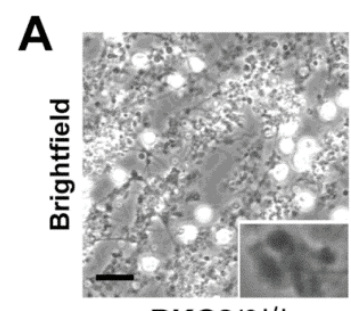

PKC $\delta / \theta^{+/+}$

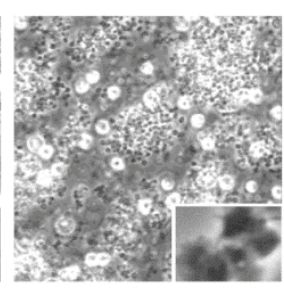

PKC ${ }^{-1-}$

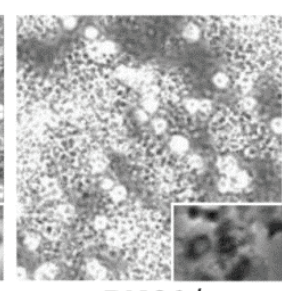

PKC $\theta^{-1-}$

B

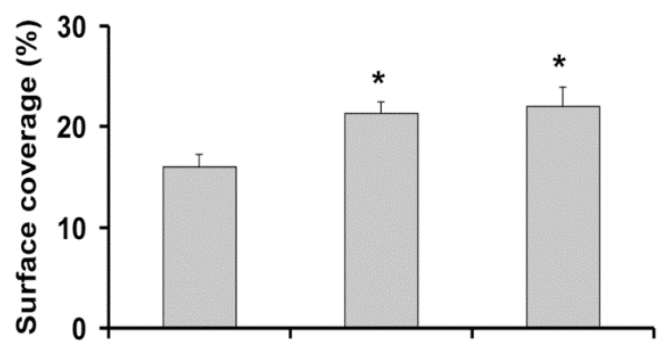

C

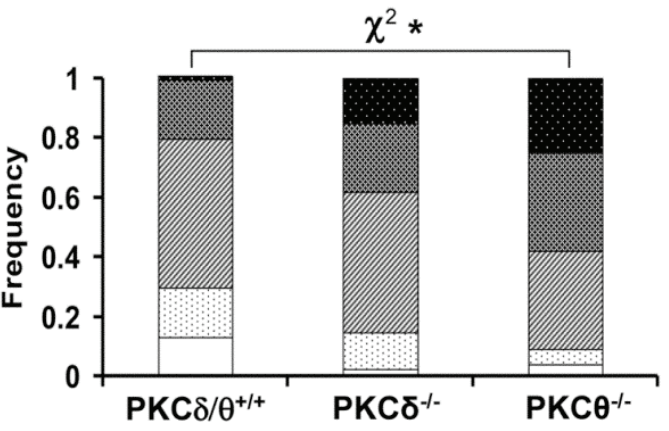

$\square 1$ to 3 plt $\square 3$ to 8 plt 8 to 40 plt 200 to 130 plt $\mathbf{a}>130$ plt

Figure 4. Deficiency of PKC $\delta$ or $\theta$ increases thrombus formation on collagen under flow. Blood from $\mathrm{PKC}^{-/}$, $\mathrm{PKC}{ }^{-/}$or matched wildtype mice was flowed over collagen (see Fig. 1). A, Representative contrast images after 4 min of perfusion (bars $20 \mu \mathrm{m}$, inserts $5 \times$ magnified). B, Quantification of platelet deposition on collagen surface. C, Morphometric analysis of aggregates on collagen surface with indicated numbers of platelets per feature. Means \pm S.E.M. $(n=3-6) ;{ }^{*} p<0.01$ vs. corresponding wildtypes; $X^{2}$ test ${ }^{*} p<0.05$.

Inhibition of conventional or novel PKC isoforms differently influences collagen-induced human platelet aggregation and activation

It was important to determine whether the roles defined for the major PKC isoforms in mouse platelets could be paralleled in human platelets. We therefore studied the relative effects of isoform non-selective and isoform-selective PKC inhibitors: the bis-indolyl maleimide, Ro-318425 (non-selective PKC inhibitor); the modified bis-indolyl maleimides, Gö6976 (potency PKC $\alpha / \beta>\varepsilon$ ) and $\beta$-inhibitor (potency $P K C \beta>\alpha>\varepsilon$ ); the biphloroglucinol, 


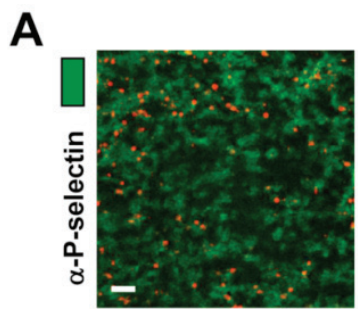

$\mathrm{PKC} \delta / \theta^{+/+}$

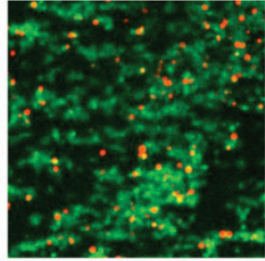

PKC $\delta^{-1-}$

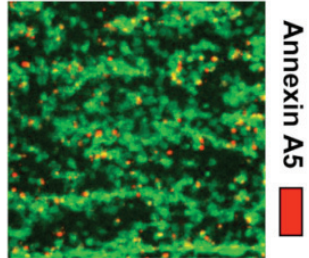

PKC $\theta^{-1-}$

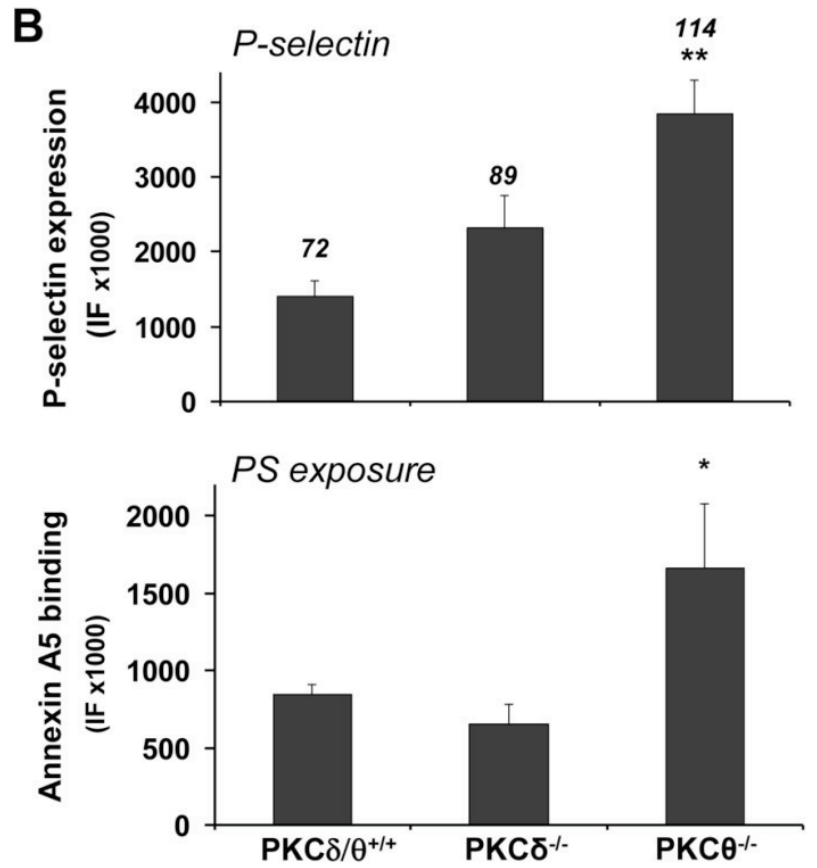

Figure 5. Deficiency of PKC $\theta$ but not $\delta$ increases collagen-induced activation of platelets in thrombi. Blood from $\mathrm{PKC}{ }^{-1}$, $\mathrm{PKC}^{-1-}$ or matched wildtype mice was flowed over collagen, and platelet thrombi were double stained with FITC-anti-mouse CD62P mAb (green) and AF647-annexin $\mathrm{A}_{5}$ (red). A, Representative confocal images $(180 \times 180 \mu \mathrm{m})$ after staining. $B$, Integrated fluorescence intensity presenting activated platelets in arbitrary units, to quantify cumulative expression of P-selectin and PS. Means \pm S.E.M. $(n=3-5) ; * * p<0.01$ and $* p<0.05$ vs. corresponding wildtypes. Numbers above each bar indicate the mean integrated pixel density of P-selectin staining, an indication of staining per platelet. For color version, see page 189 .

rottlerin (potency $\mathrm{PKC} \delta>\alpha / \beta$ ); and $\theta$-inhibitor (compound A, potency $\mathrm{PKC} \theta>\delta>\alpha / \beta$ ). As a first approach to determine the effects of inhibitors on PKC-dependent platelet function, we assessed aggregation responses to PMA. Similar to Ro-318425, which completely abolished aggregation at $0.5 \mu \mathrm{M}$, both Gö6976 and $\beta$-inhibitor abolished this response, although at higher concentrations (Supp. Fig. $1 \mathrm{~A}$ ). In contrast, rottlerin and $\theta$-inhibitor were much less inhibitory and, in the case of rottlerin, potentiated platelet aggregation to PMA. 
Similarly, Ro-318425, Gö6976 and $\beta$-inhibitor each dose-dependently suppressed PMAinduced $\alpha$ llb $\beta 3$ activation as well as $\alpha$-granule secretion (Supp. Fig. $1 B-C$ ). In contrast, $\theta$ inhibitor potentiated $\alpha \mathrm{llb} \beta_{3}$ activation, whilst rottlerin potentiated secretion. This indicates that inhibition of conventional PKC isoforms, but not novel isoforms, suppresses PMAinduced integrin activation and secretion.

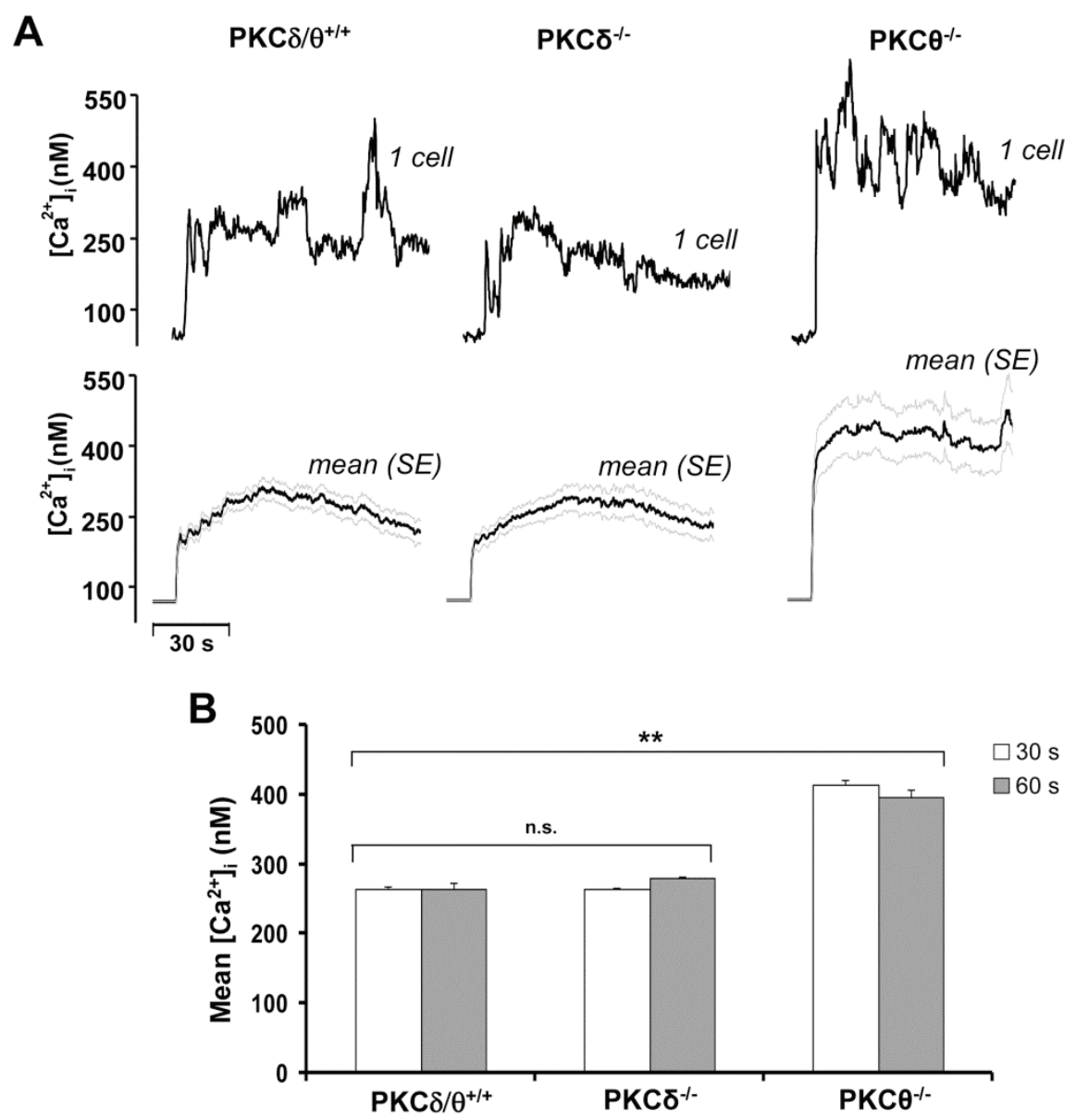

Figure 6. Deficiency of PKC $\theta$ but not $\delta$ increases collagen-induced $\mathrm{Ca}^{2+}$ signaling under flow. Whole blood containing autologous Fluo-4-loaded platelets was flowed over collagen (see Fig. 1), while fluorescence images of adhered platelets were captured in real-time $(5 \mathrm{~Hz})$. A, Traces of pseudo-ratioed changes in $\left[\mathrm{Ca}^{2+}\right]_{\mathrm{i}}$ from representative, single platelets as well as mean traces (S.E.) from overlays of $>38$ platelets. B, Graph of quantitative analysis of increased $\left[\mathrm{Ca}^{2+}\right]_{i}$ at $30 \mathrm{~s}$ (white) and $60 \mathrm{~s}$ (gray) after initial response. Data are means \pm S.E.M. $(n=38-40,4$ experiments); $* * 0<0.01$ vs. corresponding wildtypes. 

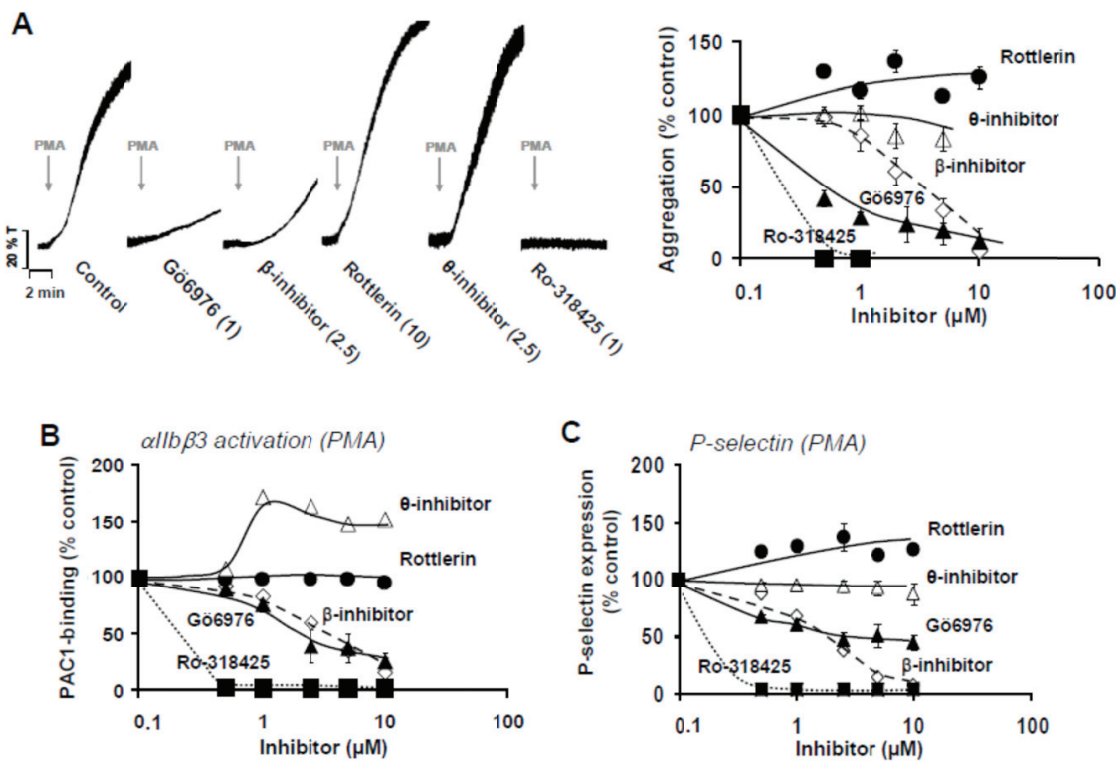

Supplementary figure 1. Inhibition of conventional or novel PKC isoforms differentially affects PMA-induced platelet activation. Washed human platelets $\left(2 \times 10^{8} / \mathrm{ml}\right)$ were pretreated with Me2SO vehicle (control), Gö6976 (blocking $P K C \alpha / \beta), \beta$-inhibitor $(P K C \beta>\alpha)$, rottlerin $(P K C \delta), \theta$-inhibitor $(P K C \theta)$, or Ro-318425 (all PKC), at a concentration of 0.5-10 $\mu \mathrm{M}$. A, Effect of inhibitors ( $\mu \mathrm{M}$ ) on platelet aggregation induced by PMA (100 nM). Shown are representative aggregation traces (optical light transmission), and dose-response curves. B-C, Inhibitor effects on platelet activation induced by PMA (100 nM), as measured by flow cytometric analysis of $\alpha \mathrm{llb} \beta_{3}$ activation and P-selectin expression. Effects are expressed as percentages of control condition. Means \pm S.E.M. $(n=3-5)$.

To assess how the inhibitors suppressed platelet PKC kinase activity, their influence on PKC exogenous substrate peptide phosphorylation was determined under the same experimental conditions ( $1 \times 10^{8}$ platelets/ml, no plasma). Whilst Ro-318425 (10 $\left.\mu \mathrm{M}\right)$ abolished kinase activity to $2.1 \pm 0.5 \%(n=5)$ of control, Gö6976 (1 $\mu \mathrm{M})$ and $\beta$-inhibitor $(2.5 \mu \mathrm{M})$ reduced phosphorylation to $54 \pm 4 \%$ and $38 \pm 6 \%$, respectively. The inhibitors of novel PKC isoforms, rottlerin $(10 \mu \mathrm{M})$ and $\theta$-inhibitor $(2.5 \mu \mathrm{M})$ reduced PKC activity to $63 \pm 8 \%$ and $75 \pm 5 \%$ of control, respectively. Combinations of inhibitors fully suppressed substrate peptide phosphorylation. These data are in agreement with previously published data (22), and demonstrate that the classical isoforms of PKC represent the more major component of platelet PKC activity in human platelets. 

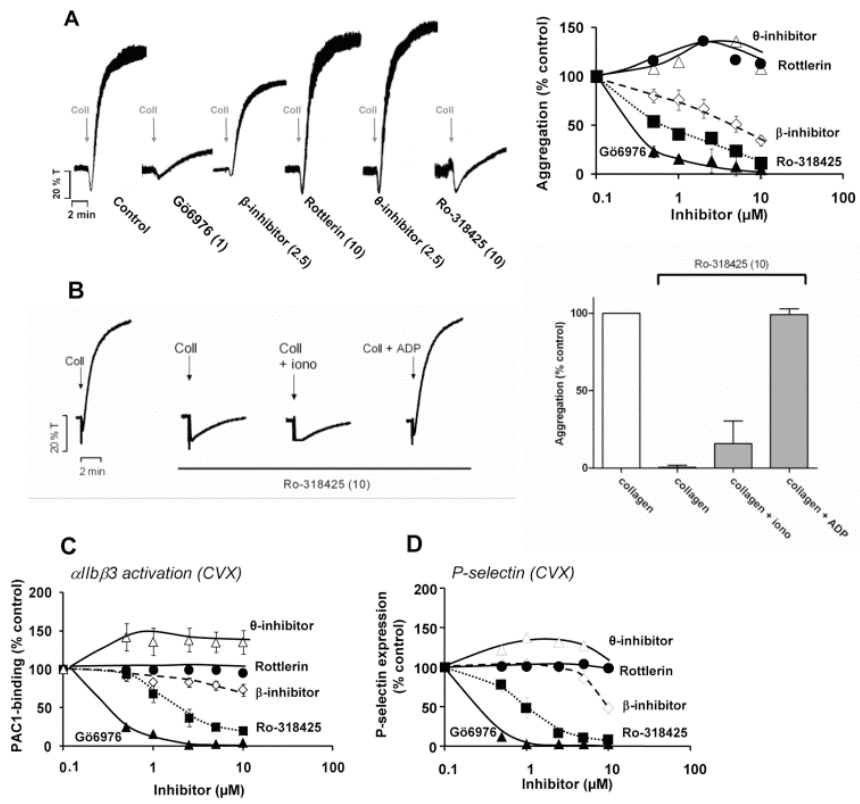

\section{D}
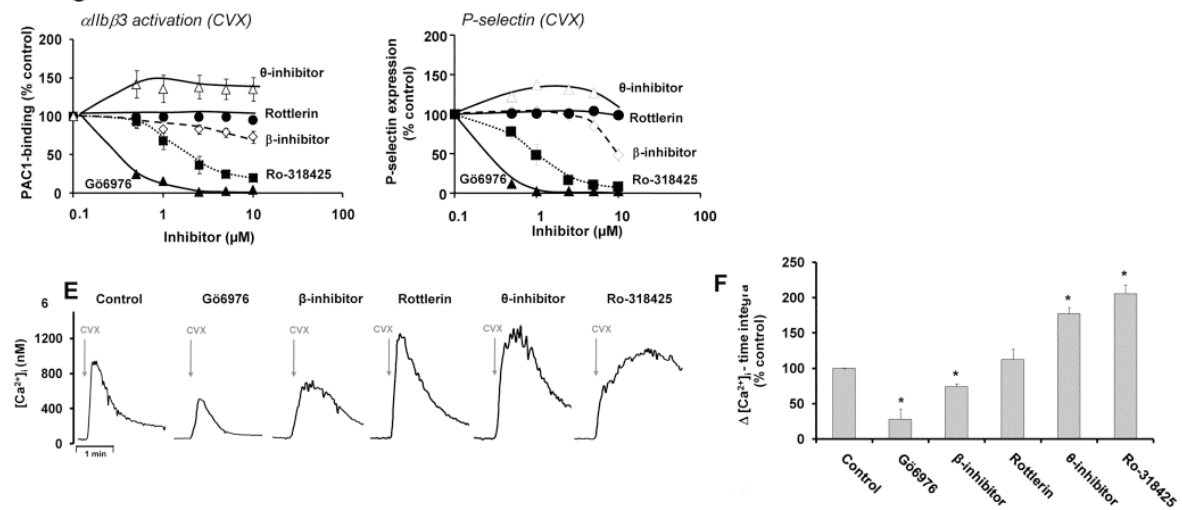

Figure 7. Inhibition of conventional or novel PKC isoforms differentially affects collagen-induced platelet activation and calcium signaling. A-D: Washed human platelets were pretreated as described for Supplementary Fig. 1, and stimulated with collagen $(5 \mu \mathrm{g} / \mathrm{ml})$ or convulxin $(70 \mathrm{ng} / \mathrm{ml})$. A, Effect of inhibitors $(\mu \mathrm{M})$ on collagen-induced platelet aggregation. Shown are representative aggregation traces (optical light transmission), and dose-response curves. B, Rescue of collagen-induced aggregation by $\operatorname{ADP}(10 \mu \mathrm{M})$ but not ionomycin (iono; $100 \mathrm{nM}$ ). Shown are representative aggregation traces and mean \pm S.E.M. $(n=4)$. C-D, Inhibitor effects on platelet activation induced by convulxin ( $c v x)$, as measured by flow cytometric analysis of $\alpha \mathrm{llb} \beta_{3}$ activation and P-selectin expression. Effects are expressed as percentages of control condition. Means \pm S.E. $(n=3-5)$. E \& F: Washed human platelets $\left(1 \times 10^{8} / \mathrm{ml}\right)$, loaded with Fura-2, were treated with Me2SO vehicle (control), Gö6976 (1.0 $\mu \mathrm{M})$, $\beta$-inhibitor $(2.5 \mu \mathrm{M})$, rottlerin

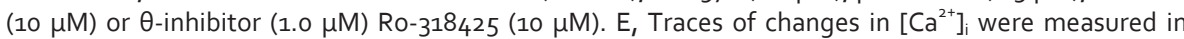
response to convulxin ( $\mathrm{cvx}, 70 \mathrm{ng} / \mathrm{ml}) . \mathrm{F}$, Quantitative effect on convulxin-induced $\left[\mathrm{Ca}^{2+}\right]_{i}$-time integrals. Means \pm S.E.M. $(n=3-4) ; * p<0.05$ vs. control condition.

Subsequently, we determined the influence of these compounds on human platelet activation via the collagen receptor, GPVI. Collagen-induced platelet aggregation was concentration-dependently inhibited by Ro-318425 (Fig. 7A) and near complete inhibition was achieved at $1 \mu \mathrm{M}$ Gö6976, while $\beta$-inhibitor was effective at higher concentrations. Ro318425 was ineffective when platelets were co-stimulated with collagen and ADP, suggesting that the inhibition of aggregation is explained by loss of granule secretion. By contrast, the calcium ionophore, ionomycin, did not rescue aggregation, suggesting that reduced 
$\mathrm{Ca}^{2+}$ signaling is not responsible for the inhibition of aggregation (Fig. $7 \mathrm{~B}$ ). Inhibition of novel PKC isoforms with rottlerin or $\theta$-inhibitor enhanced convulxin-induced platelet aggregation. In general, measurements of convulxin-induced $\alpha$ llb $\beta_{3}$ activation and P-selectin expression showed similar effects with these inhibitors (Fig. 7C-D). However, strikingly, $\theta$ inhibitor but not rottlerin markedly increased these responses, in agreement with previously published gene knockout studies $(8,24)$.

Inhibition of conventional or novel PKC isoforms differently influences GPVI-induced $\mathrm{Ca}^{2+}$ signaling

Finally, we measured GPVI-induced $\mathrm{Ca}^{2+}$ responses in washed human platelets. Gö6976 and $\beta$-inhibitor suppressed the $\left[\mathrm{Ca}^{2+}\right]_{i}$ peak and $\left[\mathrm{Ca}^{2+}\right]_{i}$-time integral (Fig. $7 \mathrm{E}-\mathrm{F}$ ), while rottlerin was without effect, and pretreatment with $\theta$-inhibitor markedly increased the platelet $\mathrm{Ca}^{2+}$ signal. This resembled the potentiatory effect seen with Ro-318425, and also noted in earlier studies with human platelets (17). Hence, the enhancement of $\mathrm{Ca}^{2+}$ signaling by general PKC inhibitors is mimicked only by inhibition of the novel PKC $\theta$ isoform. Under these conditions, convulxin ( $70 \mathrm{ng} / \mathrm{ml} ; 5 \mathrm{~min}$ ) did not cause shedding of GPVI Although treatment of platelets with a high concentration of convulxin $(250 \mathrm{ng} / \mathrm{ml})$ for 1 hour induced approximately $50 \%$ GPVI shedding, this was not affected by treatment with Ro-318425 did not affect this (Suppl. Fig. 2), suggesting that differences in $\mathrm{Ca}^{2+}$ signaling were not caused by regulated shedding of GPVI.

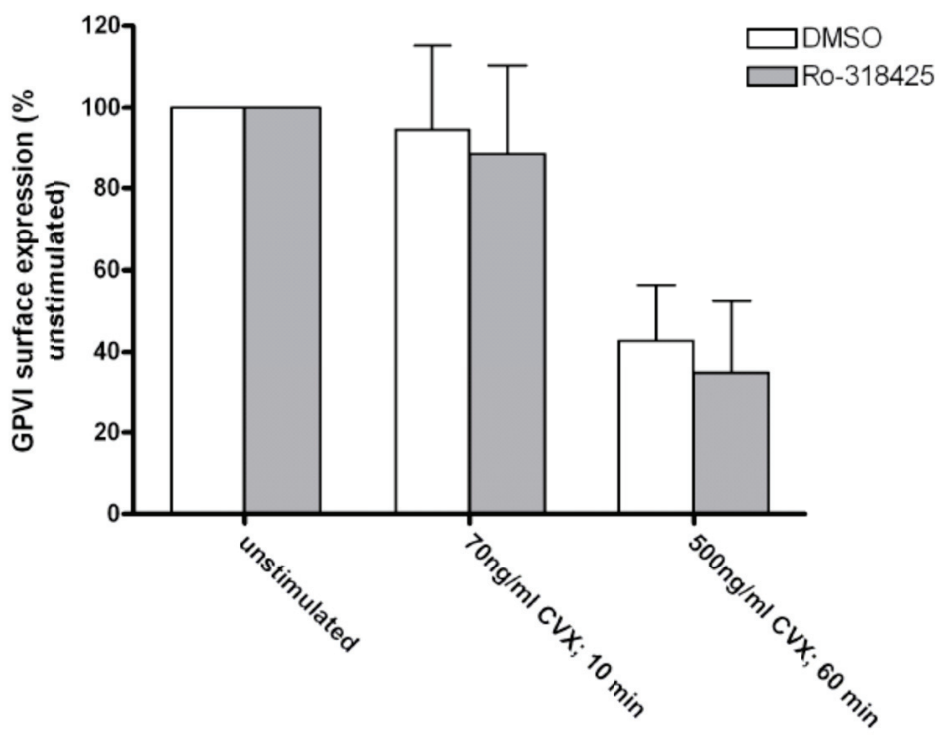

Supplementary figure 2. GPVI surface expression in washed human platelets was monitored by flow cytometry. Platelets were treated with Ro-318425 (10 $\mu \mathrm{M})$ or vehicle control (DMSO) then stimulated with convulxin (CVX) as indicated. No significant difference was seen between DMSO- or Ro318425-treated platelets $(n=4)$. 
Relative contribution of conventional and novel PKC isoforms to secretion and $\mathrm{Ca}^{2+}$ signaling Since PKC $\alpha$ and PKC $\theta$ regulate $\alpha$-granule secretion in opposite directions, we investigated which isoform was dominant during platelet activation by treating $\mathrm{PKC}{ }^{+/+}$and $\mathrm{PKC}^{-1-}$ platelets with $\theta$-inhibitor, and measured P-selectin expression by flow cytometry. Consistent with the above data, P-selectin expression was significantly inhibited in $\mathrm{PKC}^{-1-}$ platelets. Interestingly, whereas $\theta$-inhibitor slightly increased the P-selectin expression in $\mathrm{PKC} \alpha^{+/+}$platelets, there was little effect of $\theta$-inhibitor on $\mathrm{PKC} \alpha^{-/-}$platelets (Fig. 8A). These data suggest that the conventional isoforms are essential for secretion whereas PKC $\theta$ regulates the extent of secretion.

A

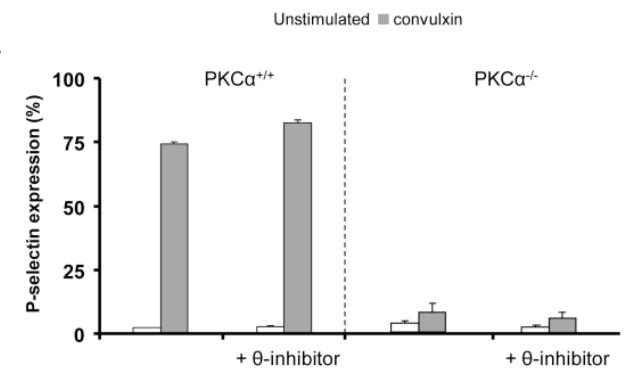

B
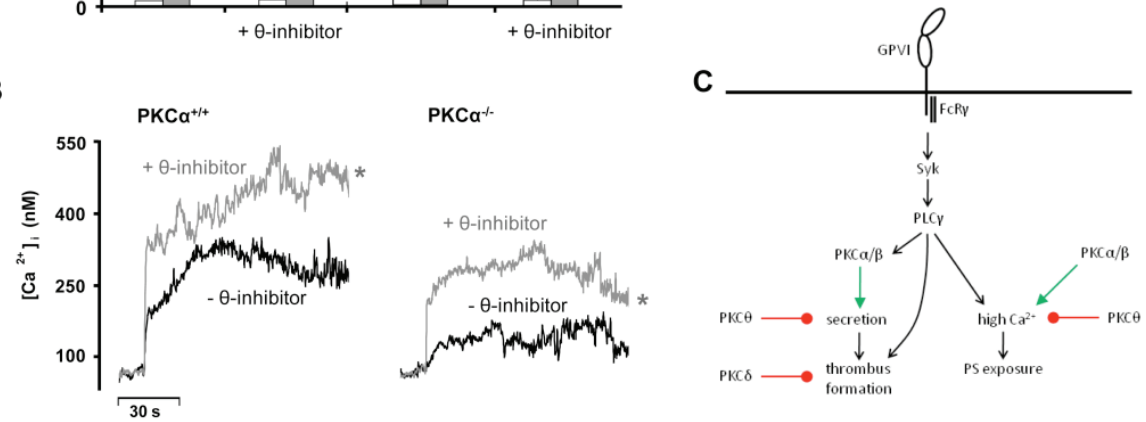

Figure 8. Relative contributions of $\mathrm{PKC} \alpha$ and $\mathrm{PKC} \theta$ to secretion and $\mathrm{Ca}^{2+}$ signaling. $\mathrm{A}$, Washed $\mathrm{PKC}^{+/+}$or $^{-1 /}$ platelets, were pretreated with $\theta$-inhibitor or vehicle control were stimulated with convulxin $(70 \mathrm{ng} / \mathrm{ml})$. $\alpha$-granule secretion was assessed by flow cytometric analysis of P-selectin expression. Data are expressed as \% cells expressing surface P-selectin $(n=3)$. B, Blood from $\mathrm{PKC}^{+/+}$or $^{-1-}$ mice containing autologous Fluo-4-loaded platelets was treated with $\theta$-inhibitor or vehicle control then flowed over collagen, as described for Fig. 3. Representative traces are shown $(n=3)$. ${ }^{*} p<0.05 \theta$ inhibitor vs. untreated condition. C, Summary diagram of the roles of different PKC isoforms in platelet secretion, thrombus formation, sustained $\mathrm{Ca}^{2+}$ signaling and PS exposure. PKC $\alpha / \beta$ are necessary for collagen-induced granule secretion, which is negatively regulated by PKC $\theta$. In the absence of $P K C \alpha / \beta$ there is no granule secretion and so no effect of $P K C \theta$. $P K C \alpha / \beta$ positively regulate sustained $\mathrm{Ca}^{2+}$ signaling and PS exposure, whereas it is negatively regulated by PKC $\theta$ even in the absence of PKC $\alpha$. PKC $\delta$ negatively regulates thrombus formation independently of granule secretion, and does not regulate $\mathrm{Ca}^{2+}$ signaling or PS exposure.

$\mathrm{PKC} \alpha$ and $\mathrm{PKC} \theta$ also regulate $\mathrm{Ca}^{2+}$ signaling under flow conditions in opposite directions. Treatment with $\theta$-inhibitor enhanced the $\mathrm{Ca}^{2+}$ signal in platelets flowed over collagen in $\mathrm{PKC}^{+/+}$platelets, consistent with the data obtained from $\mathrm{PKC}{ }^{-/}$platelets. Moreover, $\theta$ inhibitor also enhanced the $\mathrm{Ca}^{2+}$ signal in $\mathrm{PKC} \alpha^{-1-}$ platelets (Fig. 8B). These data suggest that $\mathrm{PKC} \theta$ is a more important regulator of $\mathrm{Ca}^{2+}$ signaling than of granule secretion. 


\section{Discussion}

Here, for the first time, we have addressed all four of the major platelet-expressed PKC isoforms, determining their comparative roles in regulating platelet activation by collagen under physiological flow conditions. The study is also the first to determine the role of individual PKC isoforms in regulating calcium responses, at the single cell level, in growing thrombi visualized in real time. Importantly the study reveals major positive roles for the conventional isoforms, $\mathrm{PKC} \alpha$ and $\mathrm{PKC} \beta$, in mediating thrombus formation. The absence of either isoform leads to marked suppression of secretion of $\alpha$-granules, aggregate formation, calcium signaling and PS exposure under flow. The data therefore suggest essential but non-redundant roles for these kinases in regulating these events. In contrast, absence of the novel isoforms PKC $\theta$ and PKC $\delta$ however lead to enhanced thrombus formation on collagen. The mechanisms for these isoforms are also distinct and non-redundant, since although absence of PKCO also leads to enhanced secretion, calcium signaling and phosphatidylserine exposure, absence of $\mathrm{PKC} \delta$ does not potentiate any of these functions. We conclude that all four major expressed PKC isoforms play distinct non-redundant roles, where the conventional PKCs promote and the novel PKCs inhibit thrombus formation on collagen, by a variety of mechanisms (summarized in Fig. 8C).

The studies here with mice lacking PKC $\alpha$ or PKC $\beta$ demonstrated that platelet thrombi formed on collagen were significantly smaller in comparison to wild-type thrombi. This reduced thrombus formation was associated with impaired GPVI-induced $\alpha$-granule secretion. A key role for murine PKC $\alpha$ in exocytosis of platelet $\alpha$ - and dense granules has recently been demonstrated (18). Importantly, these effects were not caused by diminished primary adhesion to collagen, since time lapse studies with Fluo-4-loaded platelets showed normal adhesion under shear in case of PKC $\alpha$ or $P K C \beta$ deficiency. Instead, our data suggest that the defect is in the ability of platelets to form aggregates on platelets that have already adhered.

In addition, in both $\mathrm{PKC} \alpha^{-/-}$and $\mathrm{PKC} \beta^{-/-}$mice, the procoagulant activity of collagenadhered platelets was diminished, consistent with the reduced $\mathrm{Ca}^{2+}$ signal. Earlier, it had been shown that the platelet procoagulant response is a direct consequence of GPVIinduced activation of collagen-adhered platelets $(42,43)$. How PKC $\alpha$ and $\beta$ regulate $\mathrm{Ca}^{2+}$ signaling is yet understood, although a role for conventional PKCs in store-operated calcium entry has previously been proposed on the basis of pharmacological studies in human platelets (44). Together, these data support the concept that both conventional PKC isoforms positively regulate thrombus formation by enhancing GPVI-induced platelet activation, leading to secretion and procoagulant activity. Interestingly, there is a high degree of nonredundancy in this process, indicating that PKCs $\alpha$ and $\beta$ may each play essential, but distinct, roles.

It was important to extend the studies in mouse platelets by studies with human platelets, using the compounds Gö6976 and $\beta$-inhibitor. Dose-response curves showed an inhibitory effect of both compounds on collagen-induced platelet aggregation as well as on GPVIinduced integrin activation and P-selectin expression. In addition, these compounds diminished GPVI-induced $\mathrm{Ca}^{2+}$ signal generation. The reduction in aggregration is likely to be 
mainly caused by loss of granule secretion, as full aggregation could be restored by costimulation with ADP. This is consistent with our previous report that coinfusion with ADP could restore thrombus formation on collagen in $\mathrm{PKC}^{-/}$deficient platelets. By contrast, enhancing cytosolic $\mathrm{Ca}^{2+}$ by costimulation with ionomycin did not rescue collagen-inducing aggregation.

For mouse platelets, the present data indicate that both isoforms are required for full GPVI-dependent activation, suggesting a non-redundancy in function, particularly for regulation of P-selectin expression and annexin $\mathrm{V}$ binding, which are both markedly suppressed in the absence of either PKC $\alpha$ or PKC $\beta$. For human platelets, the studies with Gö6976 and $\beta$ inhibitor point to a substantial reduction in platelet responses. The $\beta$-inhibitor is however much less effective than Gö6976 on convulxin-induced responses (Fig. 7A-C). This may suggest that, in human platelets, there is more redundancy of function between PKC $\alpha$ and $\mathrm{PKC} \beta$ than in mouse platelets. It may however be a reflection of the selectivity profile of these inhibitors, and may also reflect differences between the effects of gene deletion versus pharmacological inhibition of a kinase. In support of the concept that both PKC $\alpha$ and $\beta$ positively contribute to human platelet activation, are the findings in the literature that purified PKC $\alpha$ mediates granule secretion (19), PKC $\alpha$ is phosphorylated upon GPVI stimulation (45), and that PKC in general and PKC $\beta$ in particular is implicated in $\alpha$ llb $\beta_{3}$ signaling $(22,46)$.

In contrast, the current studies with mice deficient in $\mathrm{PKC} \theta$ point to a negative role in collagen-dependent granule secretion and thrombus formation. In addition, increased GPVI-induced activation of PKC ${ }^{-/}$platelets was apparent from the higher $\mathrm{Ca}^{2+}$ responses of adhered single platelets, increased numbers of procoagulant, PS-exposing cells. Consistent with these data, the $\theta$-inhibitor increased GPVI-induced human platelet aggregation as well as $\alpha$ llb $\beta_{3}$ activation, $\mathrm{Ca}^{2+}$ mobilization and $\alpha$-granule secretion. There are discrepancies in the literature about negative or positive roles for PKC $\theta$ in regulating platelet function $(24,25,47,48)$, which may be a result of differences in platelet preparation conditions. We have however previously shown platelet responses to GPVI activation to be enhanced in the absence of PKC $(24,48)$, and taken together these data would support the concept that PKC $\theta$ may down-regulate rather than up-regulate GPVI-mediated granule secretion and aggregate formation under physiological flow conditions. Mouse platelets express relatively high amounts of PKC $\theta$ compared to the other novel PKC isoforms, PKC $\delta$ and $\varepsilon(7,24)$. Furthermore, all three isoforms are phosphorylated on tyrosine following GPVI stimulation $(7,45,47,49,50)$.

In addition, the present data suggest that $\mathrm{PKC} \theta$ is the principal isoform mediating the earlier recognized effect of PKC in down-regulating GPVI-induced platelet $\mathrm{Ca}^{2+}$ signaling and procoagulant activity (17). The mechanism is currently unknown, although PKC may reduce phospholipase $\mathrm{C}$ activation (51) or increase $\mathrm{Ca}^{2+}$ extrusion via the plasma membrane $\mathrm{Ca}^{2+}$ ATPase $(14,15)$.

The enhanced thrombus formation that we report in $\mathrm{PKC}{ }^{-/}$blood is in apparent contrast to a previous report (25), in which $\mathrm{PKC} \theta^{-/}$mice showed reduced thrombus formation in vivo, in a $\mathrm{FeCl}_{3}$-induced carotid injury model. This is likely to reflect the additional contribu- 
tion of thrombin generation in vivo. Importantly, although PKC $\theta$ negatively regulates collagen-dependent platelet activation, it appears to have a positive role in thrombin-induced signaling $(25,52)$. This agonist-dependent difference is similar to that proposed by Kunapuli and colleagues for PKC $\delta$ (23). The relative importance of collagen and thrombin in vivo appear to depend on the injury model used and the extent of injury (53). We therefore suggest that the effect seen in vivo is a combination of enhanced collagen-dependent platelet activation and reduced thrombin-dependent signaling.

A tendency to increased thrombus formation was also seen with $\mathrm{PKC} \delta^{-1-}$ mice in the present study. Although some reports suggest that GPVI-induced granule secretion is increased in $\mathrm{PKC} \delta^{-/-}$mice (23), we have not been able to show this (present study and (8)), but rather demonstrate a potentiated aggregation response to collagen through enhanced filopodia formation in the absence of $\mathrm{PKC} \delta$. Consistent with this, we found with human platelets that rottlerin had little effect on collagen-induced integrin $\alpha$ llb $\beta_{3}$ activation and $\alpha$ granule secretion, but enhanced platelet aggregation. Therefore, although PKC $\theta$ and PKC $\delta$ negatively regulate collagen-dependent thrombus formation, they act through distinct mechanisms.

Treatment of human platelets with broad-spectrum PKC inhibitors blocks granule secretion and formation of large aggregates, but also enhances platelet $\mathrm{Ca}^{2+}$ signaling and PS exposure (17). This suggests that the major regulators of granule secretion and thrombus formation are $P K C \alpha$ and $P K C \beta$, since inhibition of all PKC isoforms replicates the phenotype of $\mathrm{PKC} \alpha^{-/}$or $\mathrm{PKC} \beta^{-1-}$ platelets. In contrast, the major regulator of $\mathrm{Ca}^{2+}$ signaling and $\mathrm{PS}$ exposure appears to be $P K C \theta$, since absence or pharmacological inhibition of PKC $\theta$ has a similar effect on $\mathrm{Ca}^{2+}$ signaling and PS exposure to broad-spectrum PKC inhibition.

We tested this directly by examining the effect of $\theta$-inhibitor on $\mathrm{PKC}^{-/-}$platelets. Importantly, combined loss of PKC $\alpha$ and PKC $\theta$ signaling produced a phenotype that resembled $\mathrm{PKC} \alpha^{--}$platelets for secretion, and $\mathrm{PKC}{ }^{-/-}$platelets for $\mathrm{Ca}^{2+}$ signaling. Moreover, the phenotype resembled that of platelets treated with a broad-spectrum PKC inhibitor. We propose that the conventional PKC isoforms are essential for collagen-induced granule secretion, whereas PKCO negatively regulates the extent of this secretion. This means that in the absence of conventional PKC signaling there is no granule secretion for PKC $\theta$ to regulate (Fig. 8C). In contrast, we suggest that collagen-induced sustained $\mathrm{Ca}^{2+}$ signals and subsequent PS exposure are positively regulated by PKC $\alpha$ and $\beta$ and negatively regulated by PKC $\theta$. Unlike secretion, however, the conventional isoforms are not essential for generation of the $\mathrm{Ca}^{2+}$ signal.

The data presented here are the first comparative analysis of platelet PKCs in thrombus formation on collagen, and the first to determine calcium responses, secretion and procoagulant activity at the single cell level in the growing thrombus, in platelets lacking specific PKC isoforms. Together, the data form a comprehensive analysis of the roles played by PKCs $\alpha, \beta, \delta$, and $\theta$, and reveal important distinctions in mechanism and function of these kinases. The data will provide a platform for future exploitation of these different family members in modulation of platelet function and thrombus formation. 


\section{Acknowledgements}

We thank Elizabeth Aitken for expert technical assistance supporting this work. We thank Dr Mark Jepson and Alan Leard for their assistance within the Bristol School of Medical Sciences Wolfson Bioimaging Facility. We acknowledge Boehringer Ingelheim Pharmaceuticals for the kind gift of PKC $\theta$-inhibitor. We are grateful for the financial support provided by the Marie Curie program EST 2005-020706-2 and ZonMW 11.400.0076, to JWMH, and by the British Heart Foundation for programme grant support, RG/05/015, to AWP.

\section{References}

$1 \quad$ Parker PJ \& Murray-Rust J. PKC at a glance. J Cell Sci 117, 131-2 (2004).

2 Khan WA, Blobe G, Halpern A, Taylor W, Wetsel WC, Burns D, Loomis C, \& Hannun YA. Selective regulation of protein kinase $C$ isoenzymes by oleic acid in human platelets. J Biol Chem 268, 5063-8 (1993).

3 Wang F, Naik UP, Ehrlich YH, Freyberg Z, Osada S, Ohno S, Kuroki T, Suzuki K, \& Kornecki E. A new protein kinase $C, n P K C \eta$, and $n P K C \theta$, are expressed in human platelets: involvement of $\mathrm{nPKC} \eta$ and nPKC $\theta$ in signal transduction stimulated by PAF. Biochem Biophys Res Commun 191, 240-6 (1993).

4 Crabos M, Imber R, Woodtli T, Fabbro D, \& Erne P. Different translocation of three distinct PKC isoforms with tumor-promoting phorbol ester in human platelets. Biochem Biophys Res Commun 178, 878-83 (1991).

5 Baldassare JJ, Henderson PA, Burns D, Loomis C, \& Fisher GJ. Translocation of protein kinase C isozymes in thrombin-stimulated human platelets. Correlation with 1,2-diacylglycerol levels. J Biol Chem 267, 15585-90 (1992).

6 Grabarek J, Raychowdhury M, Ravid K, Kent KC, Newman PJ, \& Ware JA. Identification and functional characterization of protein kinase $C$ isozymes in platelets and HEL cells. J Biol Chem 267, 10011-7 (1992).

7 Pears CJ, Thornber K, Auger JM, Hughes CE, Grysielska B, Protty MB, Pearce AC, \& Watson SP Differential roles of the $\mathrm{PKC}$ novel isoforms, $\mathrm{PKC} \delta$ and $\mathrm{PKC} \varepsilon$, in mouse and human platelets. Plos One 3, e3793 (2008).

8 Pula G, Schuh K, Nakayama K, Nakayama KI, Walter U, \& Poole AW. PKC $\delta$ regulates collageninduced platelet aggregation through inhibition of VASP-mediated filopodia formation. Blood 108, 4035-44 (2006).

9 Shattil SJ \& Brass LF. Induction of the fibrinogen receptor on human platelets by intracellular mediators. J Biol Chem 262, 992-1000 (1987).

10 Toullec D, Pianetti P, Coste H, Bellevergue P, Grand-Perret T, Ajakane M, Baudet V, Boissin P,

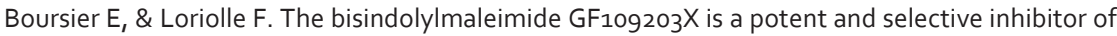
protein kinase C. J Biol Chem 266, 15771-81 (1991).

11 Walker TR \& Watson SP. Synergy between $\mathrm{Ca}^{2+}$ and protein kinase $\mathrm{C}$ is the major factor in determining the level of secretion from human platelets. Biochem J 289, 277-83 (1993).

Hers I, Donath J, van Willigen G, \& Akkerman JW. Differential involvement of tyrosine and serine/threonine kinases in platelet integrin $\alpha$ llb $\beta_{3}$ exposure. Arterioscler Thromb Vasc Biol 18, 404-14 (1998).

13 Quinton TM, Kim S, Dangelmaier C, Dorsam RT, Jin J, Daniel JL, \& Kunapuli SP. Protein kinase Cand calcium-regulated pathways independently synergize with $\mathrm{Gi}$ pathways in agonist-induced fibrinogen receptor activation. Biochem J 368, 535-43 (2002).

14 Cavallini L \& Alexandre A. $\mathrm{Ca}^{2+}$ efflux from platelets: control by protein kinase $\mathrm{C}$ and the filling state of the intracellular $\mathrm{Ca}^{2+}$ stores. Eur J Biochem 222, 693-702 (1994).

15 Rink TJ \& Sage SO. Calcium signaling in human platelets. Annu Rev Physiol 52, 431-49 (1990).

16 Hardy AR, Conley PB, Luo J, Benovic JL, Poole AW, \& Mundell SJ. P2 11 and P2 12 receptors for ADP desensitize by distinct kinase-dependent mechanisms. Blood 105, 3552-60 (2005).

17 Strehl A, Munnix IC, Kuijpers MJ, van der Meijden PE, Cosemans JM, Feijge MA, Nieswandt B, \& Heemskerk JW. Dual role of platelet protein kinase $C$ in thrombus formation: stimulation of pro- 
aggregatory and suppression of procoagulant activity in platelets. J Biol Chem 282, 7046-55 (2007).

18 Konopatskaya O, Gilio K, Harper MT, Cosemans JM, Karim AZ, Whiteheart SW, Molkentin JD, Verkade P, Heemskerk JW, \& Poole AW. PKC $\alpha$ is a critical regulator of platelet function and thrombus formation. J Clin Invest in press 119, 399-407 (2009).

19 Yoshioka A, Shirakawa R, Nishioka H, Tabuchi A, Higashi T, Ozaki H, Yamamoto A, Kita T, \& Horiuchi $\mathrm{H}$. Identification of protein kinase $\mathrm{C} \alpha$ as an essential, but not sufficient, cytosolic factor for $\mathrm{Ca}^{2+}$-induced alpha- and dense-core granule secretion in platelets. J Biol Chem 276, 39379-85 (2001).

20 Tabuchi A, Yoshioka A, Higashi T, Shirakawa R, Nishioka H, Kita T, \& Horiuchi H. Direct demonstration of involvement of protein kinase $\mathrm{C} \alpha$ in the $\mathrm{Ca}^{2+}$-induced platelet aggregation. J Biol Chem 278, 26374-9 (2003).

Konopatskaya O \& Poole AW. Protein kinase Calpha: disease regulator and therapeutic target. Trends in Pharmacological Sciences 31, 8-14 (2010).

22 Buensuceso CS, Obergfell A, Soriani A, Eto K, Kiosses WB, Arias-Salgado EG, Kawakami T, \& Shattil SJ. Regulation of outside-in signaling in platelets by integrin-associated protein kinase $C \beta$. J Biol Chem 280, 644-53 (2005).

23 Chari R, Getz T, Nagy B, Bhavaraju K, Mao Y, Binagari YS, Muruggappam S, Nakayama K, \& Kunapuli SP. Protein kinase $C \delta$ differentially regulates platelet functional responses. Arterioscler Thromb Vasc Biol 29, 699-705 (2009).

24 Hall KJ, Harper MT, Gilio K, Cosemans JM, Heemkerk JW, \& Poole AW. Genetic analysis of the role of protein kinase $C \theta$ in platelet function and thrombus formation. Plos One 3, e3277/1-e/7 (2008).

25 Nagy B, Bhavaraju K, Getz T, Bynagari YS, Kim S, \& Kunapuli SP. Impaired activation of platelets lacking protein kinase $\mathrm{C} \theta$ isoform. Blood 113, 2557-67 (2009).

26 Braz JC, Gregory K, Pathak A, Zhao W, Sahin B, Klevitsky R, Kimball TF, Lorenz JN, Nairn AC, Liggett SB, Bodi I, Wang S, Schwartz A, Lakatta EG, DePaoli-Roach AA, Robbins J, Hewett TE, Bibb JA, Westfall MV, Kranias EG, \& Molkentin JD. PKC $\alpha$ regulates cardiac contractility and propensity toward heart failure. Nat Med 10, 248-53 (2004).

27 Standaert ML, Bandyopadhyay G, Galloway L, Soto J, Ono Y, Kikkawa U, Farese RV, \& Leitges M. Effects of knockout of the protein kinase $C \beta$ gene on glucose transport and glucose homeostasis. Endocrinology 140, 4470-7 (1999).

28 Miyamoto A, Nakayama K, Imaki H, Hirose S, Jiang Y, Abe M, Tsukiyama T, Nagahama H, Ohno S, S. H, \& Nakayama KI. Increased proliferation of B cells and auto-immunity in mice lacking protein kinase $C \delta$. Nature 416, 865-9 (2002).

29 Sun Z, Arendt CW, Ellmeier W, Schaeffer EM, Sunshine MJ, Gandhi L, Annes J, Petrzilka D, Kupfer A, Schwartzberg PL, \& Littman DR. PKCO is required for TCR-inducedNF-kappaB active tion in mature but not immature T lymphocytes. Nature 404, 402-7 (2000).

30 Cywin CL, Dahmann G, Prokopowicz AS, Young ER, Magolda RL, Cardozo MG, Cogan DA, Disalvo D, Ginn JD, Kashem MA, Wolak JP, Homon CA, Farrell TM, Grbic H, Hu H, Kaplita PV, Liu LH, Spero DM, Jeanfavre DD, O'Shea KM, White DM, Woska JR, \& Brown ML. Discovery of potent and selective PKC $\theta$ inhibitors. Bioorg Med Chem Lett 17, 225-30 (2007).

31 Siljander P, Farndale RW, Feijge MA, Comfurius P, Kos S, Bevers EM, \& Heemskerk JW. Platelet adhesion enhances the glycoprotein VI-dependent procoagulant response: involvement of p38 MAP kinase and calpain. Arterioscler Thromb Vasc Biol 21, 618-27 (2001).

32 Kuijpers MJ, Schulte V, Bergmeier W, Lindhout T, Brakebusch C, Offermanns S, Fässler R, Heemskerk JW, \& Nieswandt B. Complementary roles of glycoprotein VI and $\alpha_{2} \beta_{1}$ integrin in collagen-induced thrombus formation in flowing whole blood ex vivo. FASEB J 17, 685-7 (2003).

33 Van der Meijden PE, Schoenwaelder SM, Cosemans JM, Wetzker R, Heller R, Jackson SP, \& Heemskerk JW. Dual $\mathrm{P}_{2} \mathrm{Y}_{12}$ receptor signaling in thrombin-stimulated platelets: involvement of phosphoinositide 3-kinase $\beta$ but not $\gamma$ isoforms in $\mathrm{Ca}^{2+}$ mobilization and procoagulant activity. FEBS J 275, 371-85 (2008).

34 Siljander PRM, Munnix ICA, Smethurst PA, Deckmyn H, Lindhout T, Ouwehand WH, Farndale RW, \& Heemskerk JWM. Platelet receptor interplay regulates collagen-induced thrombus for mation in flowing human blood. Blood 103, 1333-41 (2004). 
35 Lecut C, Schoolmeester A, Kuijpers MJ, Broers JL, van Zandvoort MA, Vanhoorelbeke K, Deckmyn $\mathrm{H}$, Jandrot-Perrus $\mathrm{M}$, \& Heemskerk JW. Principal role of glycoprotein VI in $\alpha_{2} \beta_{1}$ and $\alpha$ llb $\beta_{3}$ activation during collagen-induced thrombus formation. Arterioscler Thromb Vasc Biol 24, 1727-33 (2004).

36 Munnix IC, Kuijpers MJ, Auger JM, Thomassen CM, Panizzi P, van Zandvoort MA, Rosing J, Bock $P E$, Watson SP, \& Heemskerk JW. Segregation of platelet aggregatory and procoagulant microdomains in thrombus formation. Regulation by transient integrin activation. Arterioscler Thromb Vasc Biol 27, 2484-90 (2007).

37 Auger JM, Kuijpers MJ, Senis YA, Watson SP, \& Heemskerk JW. Adhesion of human and mouse platelets to collagen under shear: a unifying model. FASEB J 19, 825-7 (2005).

38 Heemskerk JW, Willems GM, Rook MB, \& Sage SO. Ragged spiking in free calcium in ADPstimulated platelets: regulation of puff-like calcium signal in vitro and ex vivo. J Physiol 535, 62535 (2001).

39 Orr JW, Keranen LM, \& Newton AC. Reversible exposure of the pseudosubstrate domain of protein kinase $C$ by phosphatidylserine and diacylglycerol. J Biol Chem 267, 15263-6 (1992).

40 Kahn ML. Platelet-collagen responses: molecular basis and therapeutic promise. Sem Thromb Hemost 30, 419-25 (2004).

41 Savage B, Almus-Jacobs F, \& Ruggeri ZM. Specific synergy of multiple substrate-receptor interactions in platelet thrombus formation under flow. Cell 94, 657-66 (1998).

42 Heemskerk JWM, Vuist WMJ, Feijge MAH, Reutelingsperger CPM, \& Lindhout T. Collagen but not fibrinogen surfaces induce bleb formation, exposure of phosphatidylserine and procoagulant activity of adherent platelets. Evidence for regulation by protein tyrosine kinase-dependent $\mathrm{Ca}^{2+}$ responses. Blood 90, 2615-25 (1997).

43 Nieswandt B, Brakebusch C, Bergmeier W, Schulte V, Bouvard D, Mohtari-Nejad R, Lindhout T, Heemskerk JW, Zirngibl H, \& Fässler R. Glycoprotein VI but not $\alpha_{2} \beta_{1}$ integrin is essential for platelet interaction with collagen. EMBO J 20, 2120-30 (2001).

44 Harper MT \& Sage SO. PAR-1-dependent pp6osrc activation is dependent on protein kinase C and increased $\left[\mathrm{Ca}^{2+}\right]_{\mathrm{i}}$ : evidence that pp6osrc does not regulate PAR-1-dependent $\mathrm{Ca}^{2+}$ entry in human platelets. J Thromb Haemost 4, 2695-703 (2006).

45 Pula G, Crosby D, Baker J, \& Poole AW. Functional interaction of protein kinase C $\alpha$ with the tyrosine kinases Syk and Src in platelets. J Biol Chem 280, 7194-205 (2005).

46 Giuliano S, Nesbitt WS, Rooney M, \& Jackson SP. Bidirectional integrin $\alpha$ llb $\beta 3$ signalling regulating platelet adhesion under flow: contribution of protein kinase $C$. Biochem J 372, 163-72 (2003).

47 Soriani A, Moran B, De Virgilio M, Kawakami T, Altman A, Lowell C, Eto K, \& Shattil SJ. A role for $P K C \theta$ in outside-in $\alpha$ llb $\beta 3$ signaling. J Thromb Haemost 4, 648-55 (2006).

48 Harper MT \& Poole AW. PKCO in platelet activation. Blood 114, 489-91 (2009).

49 Crosby D \& Poole AW. Physical and functional interaction between protein kinase C delta and Fyn tyrosine kinase in human platelets. J Biol Chem 278, 24533-41 (2003).

50 Crosby D \& Poole AW. Interaction of Bruton's tyrosine kinase and protein kinase Ctheta in platelets. Cross-talk between tyrosine and serine/threonine kinases. J Biol Chem 277, 9958-65 (2002).

51 Murphy CT, Elmore M, Kellie S, \& Westwick J. The relationship between cytosolic $\mathrm{Ca}^{2+}$, sn-1,2diacylglycerol and inositol 1,4,5-trisphosphate elevation in platelet-activating-factor-stimulated rabbit platelets. Influence of protein kinase C on production of signal molecules. Biochem J 278 (Pt 1), 255-61 (1991).

52 Cohen S, Braiman A, Shibinsky G, Ohayon A, Altman A, \& Isakov N. PKCtheta is required for hemostasis and positive regulation of thrombin-induced platelet aggregation and alpha-granule secretion. Biochem. Biophys. Res. Commun. 385, 22-7 (2009).

53 Mangin P, Yap CL, Nonne C, Sturgeon SA, Goncalves I, Yuan Y, Schoenwaelder SM, Wright CE, Lanza F, \& Jackson SP. Thrombin overcomes the thrombosis defect associated with platelet GPVI/FcRgamma deficiency. Blood 107, 4346-53 (2006). 


\section{Chapter 6}

Roles of platelet STIMi and Orail in glycoprotein VIand thrombin-dependent procoagulant activity and thrombus formation

Gilio K, van Kruchten R, Braun A, Berna-Erro A, Feijge MA, Stegner D, van der Meijden PE, Kuijpers MJ, Varga-Szabo D, Heemskerk JW, and Nieswandt B 


\begin{abstract}
In platelets, STIM 1 has been recognized as the key regulatory protein in store-operated $\mathrm{Ca}^{2+}$ entry (SOCE) with Orai1 as principal $\mathrm{Ca}^{2+}$ entry channel. Both proteins contribute to collagen-dependent arterial thrombosis in mice in vivo. Unclear is whether STIM2 is involved. A key platelet response relying on $\mathrm{Ca}^{2+}$ entry is the surface exposure of phosphatidylserine (PS), which accomplishes platelet procoagulant activity. We studied this response in mouse platelets deficient in STIM1, STIM2 or Orai1. Upon high-shear flow of blood over collagen, $\mathrm{Stim1}^{-1-}$ and $\mathrm{Orai}^{-1-}$ platelets had greatly impaired GPVI-dependent $\mathrm{Ca}^{2+}$ signals, and they were deficient in PS exposure and thrombus formation. In contrast, Stim2 ${ }^{-1-}$ platelets reacted normally. Upon blood flow in the presence of thrombin generation and coagulation, $\mathrm{Ca}^{2+}$ signals of $\mathrm{Stim}_{1}^{-1-}$ and Orai1 $^{-1-}$ platelets were partly reduced, while the PS exposure and formation of fibrin-rich thrombi were normalized. Washed Stim ${ }^{-1-}$ and Orai $^{-1-}$ platelets were deficient in GPVI-induced PS exposure and prothrombinase activity, but not when thrombin was present as co-agonist. Markedly, SKF96365, a blocker of (receptor-operated) $\mathrm{Ca}^{2+}$ entry, inhibited $\mathrm{Ca}^{2+}$ and procoagulant responses even in $\mathrm{Stim}_{1}{ }^{-1-}$ and $\mathrm{Orai1}^{-1-}$ platelets. These data show for the first time that: (i) STIM1 and Orai1 jointly contribute to GPVIinduced SOCE, procoagulant activity and thrombus formation, (ii) a compensating $\mathrm{Ca}^{2+}$ entry pathway is effective in the additional presence of thrombin; (iii) platelets contain two mechanisms of $\mathrm{Ca}^{2+}$ entry and PS exposure, only one relying on STIM1-Orai1 interaction.
\end{abstract}

\title{
Introduction
}

In platelets, elevation in cytosolic $\left[\mathrm{Ca}^{2+}\right]_{\mathrm{i}}$ is imperative to almost all functional responses. Moderate and transient rises in $\left[\mathrm{Ca}^{2+}\right]_{\mathrm{i}}$ mediate shape change, integrin $\alpha \mathrm{llb} \beta_{3}$ activation, thromboxane formation and secretion of granule contents, while high and prolonged $\left[\mathrm{Ca}^{2+}\right]_{i}$ rises are required for the procoagulant response (1). The latter is achieved by a $\mathrm{Ca}^{2+}$. activated scramblase mechanism disturbing the normal phospholipid asymmetry in the plasma membrane, with as a result the exposure of phosphatidylserine (PS) at the outer membrane surface $(2,3)$. Exposed PS provides high-affinity binding sites for key coagulation factors and, thereby, facilitates the assembly of tenase and prothrombinase complexes, which are responsible for the formation of factor Xa and thrombin, respectively (2). Since thrombin is one of the most potent platelet agonists, the procoagulant platelet response triggers a potent positive feedback loop of platelet and coagulation activation. Recent in vivo studies have indicated that PS exposure and ensuing thrombin generation are key regulatory events in murine arterial thrombus formation $(4,5)$.

Whereas stored platelets may expose procoagulant PS in a $\mathrm{Ca}^{2+}$-independent way, PS exposure in activated platelets relies on a high and prolonged rise in cytosolic $\left[\mathrm{Ca}^{2+}\right]_{\mathrm{i}}(6)$. Platelet stimulation with single G-protein-coupled agonists, like thrombin and ADP, results in limited PS exposure $(7,8)$, but stimulation of the tyrosine kinase-linked collagen receptor glycoprotein VI (GPVI), with ligands such as collagen-related peptide (CRP) or convulxin, results in appreciable procoagulant activity $(9,10)$. Combined stimulation of the collagen and thrombin receptors though results in high PS exposure, likely because these agonists use different signaling pathways for mobilizing cytosolic $\mathrm{Ca}^{2+}(1)$. While thrombin transient- 
ly activates Gq $\alpha$ and phospholipase $C \beta_{2} / \beta_{3}$ isoforms, activation of GPVI causes a more persistent activation of the phospholipase $C_{\gamma 2}$ isoform (11). For PS exposure, entry of extracellular $\mathrm{Ca}^{2+}$ is required, complementing the $\mathrm{Ca}^{2+}$-mobilizing effect of phospholipase $\mathrm{C}$ stimulation, in order to reach sufficiently high $\left[\mathrm{Ca}^{2+}\right]_{i}(9,12,13)$.

In platelets like other cells, $\mathrm{Ca}^{2+}$ entry can be triggered by receptor stimulation as well as by $\mathrm{Ca}^{2+}$ mobilization from stores via the processes of receptor-operated $\mathrm{Ca}^{2+}$ entry (ROCE) and store-operated $\mathrm{Ca}^{2+}$ entry (SOCE), respectively (14). For long, not only the responsible $\mathrm{Ca}^{2+}$ entry channels, but also the coupling mechanisms of receptor activation and $\mathrm{Ca}^{2+}$ store depletion to channel opening have remained elusive. In earlier work with platelets, roles of the TRPC 1 and TRPC 6 channel proteins in $\mathrm{Ca}^{2+}$ entry have been proposed $(15,16)$. Recent studies however have shown the importance of the Orai class of plasma membrane $\mathrm{Ca}^{2+}$ channels. The channel Oraiı (also called CRACM1) oligomerizes and opens, following depletion of the $\mathrm{Ca}^{2+}$ stores, by interacting with $\mathrm{Ca}^{2+}$ sensing stromal-interacting molecule 1 (STIM1), which is a transmembrane protein located in the endoplasmic reticulum (17-19). The homologous protein STIM2 can have a similar regulatory role in $\mathrm{Ca}^{2+}$ entry (20). Both Orai1 and STIM1 have been implicated in the physiological activation of $\mathrm{T}$ cells and mast cells $(21,22)$. Recent studies using genetically modified mice have established that $\mathrm{STIM}_{1}$ and Orai1 account for the large majority of SOCE in platelets. The importance of this SOCE pathway appeared from the finding that platelet deficiency in either Orai1 or STIM1 protects against collagen-dependent arterial thrombus formation and brain infarction in vivo $(23,24)$. In confirmation, others have provided evidence that a functional R93W mutation in Orai1 leads to impaired GPVI-induced platelet activation (25).

In the present paper, we investigated whether the STIM isoforms and Orai1 provide the main $\mathrm{Ca}^{2+}$ entry mechanism responsible for PS exposure and procoagulant activity in platelets stimulated by the collagen and thrombin receptors. The studies were carried out using mice with $\mathrm{Stim}_{1}{ }^{--}$, Stim2 ${ }^{-1-}$, or Orai1 ${ }^{-1-}$ platelets.

\section{Experimental procedures}

\section{Mouse Strains}

Animal studies were approved by the local animal care and use committees. Mice homozygously deficient in Stimı or Orai1 were generated from embryonic stem cell clones and germline transmission, as described $(23,24)$. Since these animals suffered from early lethality and growth retardation, bone marrow chimeras were created which had normal viability. Female, 5- 6 weeks old $\mathrm{C}_{57} \mathrm{BL} / 6$ mice were irradiated with a single dose of $10 \mathrm{~Gy}$, and injected intravenously with bone marrow cells from donor $\mathrm{Stim}_{1}{ }^{-1-}$, Orail ${ }^{-1-}$ or wild type mice $(4 \times$ $10^{6}$ cells/animal). The recipient mice received acidified water containing $2 \mathrm{~g} / \mathrm{l}$ neomycin sulfate for 6 weeks after transplantation. Blood was taken from the chimeras after $>6$ weeks. Mice homozygously deficient in Stimz had a mixed genetic background and were compared with wild types of the same background (26). Blood cell counts of all mice were in the normal range. Purified platelets from (bone marrow-transplanted) mice were subjected to Western blotting to confirm knockout of STIM proteins. Deficiency in Orai1 transcripts was confirmed by RT-PCR analysis (24). 


\section{Materials}

$\mathrm{H}$-Phe-Pro-Arg chloromethyl ketone (PPACK) was obtained from Calbiochem. Annexin $\mathrm{A}_{5}$ labeled with fluorescein isothiocyanate (FITC), Fura-2 and Fluo-4 acetoxymethyl esters and pluronic F-127 were from Invitrogen. Thrombin substrate, Z-Gly-Gly-Arg aminomethyl coumarin (Z-GGR-AMC), was from Bachem; fibrillar type I collagen (Horm) from Nycomed; recombinant human tissue factor from Dade Behring. Apyrase (grade $V$ ), bovine serum albumin (BSA), heparin, SKF96365 and thrombin were from Sigma. The GPVI agonist, CRP was synthesized and cross-linked by Tana Laboratories; the agonist convulxin was purified as described (10). FITC-labeled anti-mouse P-selectin mAb was from Emfret Analytics. Bovine coagulation factors and other materials were from sources indicated before (27).

\section{Blood collection and platelet preparation}

Mouse blood was obtained via orbital puncture under anesthesia. For perfusion studies in the absence of coagulation, blood was collected into a mixture of $40 \mu \mathrm{M}$ PPACK, $5 \mathrm{U} / \mathrm{ml}$ heparin and $40 \mathrm{U} / \mathrm{ml}$ fragmin. For later perfusions with coagulation, blood was collected into 12.9 mM trisodium citrate. Citrate-anticoagulated blood was also used to prepare plateletrich plasma (PRP), normalized with autologous platelet-poor plasma (PPP) to a platelet count of $1 \times 10^{8} / \mathrm{ml}$. Washed platelets were prepared from PRP by supplementation of ACD solution (1:25). After centrifugation, platelets were suspended in Hepes buffer pH 7.45 (136 $\mathrm{mM} \mathrm{NaCl}, 5 \mathrm{mM}$ Hepes, $2.7 \mathrm{mM} \mathrm{KCl}, 2 \mathrm{mM} \mathrm{MgCl}$, $0.42 \mathrm{mM} \mathrm{Na} 2 \mathrm{HPO}_{4}, 5 \mathrm{mM}$ glucose and $0.1 \%$ BSA). Cells were counted with a Coulter counter.

\section{Activation of suspended platelets}

Washed mouse platelets were loaded with the ratiometric $\mathrm{Ca}^{2+}$ probe, Fura-2, as described (23). Platelets suspended in the presence or absence of $1 \mathrm{mM} \mathrm{CaCl}_{2}$ were preincubated with SKF96365 (100 $\mu \mathrm{M})$, and activated with collagen and/or thrombin receptor agonists, as indicated. Changes in fluorescence was measured with a PerkinElmer LS 55 fluorometer. Excitation was alternated between 340 and $380 \mathrm{~nm}$, and emission was measured at $509 \mathrm{~nm}$. Calibration parameters of nanomolar $\left[\mathrm{Ca}^{2+}\right]_{i}$ were obtained by lysis with $1 \%$ Triton $\mathrm{X}-100$ and addition of a surplus of EGTA. For flow cytometry, unloaded washed platelets $(1 \times$

$10^{8} / \mathrm{ml}$ ) were activated in the presence of $\mathrm{CaCl}_{2}$ with indicated agonists for $10 \mathrm{~min}$; stirring was absent to prevent platelet aggregation (28). Surface expression of PS was detected in the presence of $\mathrm{CaCl}_{2}(2 \mathrm{mM})$ with FITC-annexin $\mathrm{A}_{5}(0.5 \mu \mathrm{g} / \mathrm{ml})$.

\section{Thrombus formation on collagen}

Glass coverslips were coated with fibrillar type I collagen and blocked with BSA-containing Hepes buffer $\mathrm{pH}$ 7.45. The coverslips were mounted in a transparent, $50 \mu \mathrm{m}$-deep parallelplate poly (methyl) methacrylate flow chamber (29). Mouse blood was perfused through the flow device at defined shear rate under physiological, millimolar concentrations of divalent cations. For experiments in the absence of coagulation, PPACK/heparin-treated blood was flowed over collagen at $1000 \mathrm{~s}^{-1}$ during $4 \mathrm{~min}$. For experiments in the presence of coagulation, citrate-anticoagulated blood was recalcified directly before entering the flow chamber, 
using a two-pump system (28). Briefly, $1 \mathrm{ml}$ syringes were filled with citrate-anticoagulated blood or isotonic $\mathrm{CaCl}_{2} / \mathrm{MgCl}_{2}$ solution (110 mM NaCl, $13.3 \mathrm{mM} \mathrm{CaCl} 2$ and $6.7 \mathrm{mM} \mathrm{MgCl} 2$ ). The syringes were connected to the flow chamber via a v-shaped inlet, designed to give optimal fluid mixing. By co-infusing both fluids into the chamber at equal flow rate (final shear rate, $1000 \mathrm{~s}^{-1}$ ), coagulation was started by collagen- and factor XII-dependent activation (30). The thrombi on coverslips were post-labeled by perfusion with FITC-annexin $\mathrm{A}_{5}$ $(0.5 \mu \mathrm{g} / \mathrm{ml})$ in Hepes buffer $\mathrm{pH}$ 7.45, containing $2 \mathrm{mM} \mathrm{CaCl} 2$ and $1 \mathrm{U} / \mathrm{ml}$ heparin. Brightfield and fluorescence images were recorded from at least 10 randomly chosen microscopic fields (31). Images were analyzed with ImagePro software (Media Cybernetics). The procoagulant index of thrombi was determined as the ratio of surface coverage of PS-exposing platelets (FITC-annexin A5) to the coverage of total platelets (32).

\section{Single platelet $\mathrm{Ca}^{2+}$ fluxes under flow}

Mouse platelets were loaded with Fluo-4 (33), and added to PPACK/fragmin- or citrateanticoagulated blood from the same genotype (10\% labeled platelets). During blood flow over collagen, 16-bit digital fluorescence images were recorded at high-speed ( $5 \mathrm{~Hz}$ ) using an EM-CCD camera (30). Regions-of-interest representing single adhered platelets were analyzed off-line for changes in fluorescence (F) (33). Pseudo-ratio F/Fo values were converted into nanomolar concentrations of $\left[\mathrm{Ca}^{2+}\right]_{i}$ using predefined calibration parameters (34). For quantitative purposes, traces from individual cells were superimposed, so that $\left[\mathrm{Ca}^{2+}\right]_{\mathrm{i}}$ rises started at the same frame number.

\section{Prothrombinase activity}

Prothrombinase-stimulating activity was determined at linear assay conditions, as previously assessed for human platelets (35). Washed mouse platelets were diluted in Hepes buffer $\mathrm{pH} 7.45$ containing $3 \mathrm{mM} \mathrm{CaCl} 2$ to a count of $4 \times 10^{5} / \mathrm{ml}$, and incubated with $0.5 \mu \mathrm{M}$ prothrombin, $2 \mathrm{nM}$ factor $\mathrm{Va}$ and $1 \mathrm{nM}$ factor $\mathrm{Xa}\left(37^{\circ} \mathrm{C}\right)$. Samples were taken after exactly 3 min, and transferred to vials containing thrombin substrate, $\mathrm{S} 2238(0.5 \mathrm{mM})$, for chromogenic measurement of the thrombin formed.

\section{Thrombin generation}

Thrombin generation was measured in citrate-anticoagulated PRP (27). The PRP, pooled from 3 animals with the same (chimeric) genotype, was diluted with autologous PPP to a count of $1.5 \times 10^{8}$ platelets $/ \mathrm{ml}$. Samples were activated with convulxin $(100 \mathrm{ng} / \mathrm{ml})$, ionomycin $(20 \mu \mathrm{M})$ or vehicle for $15 \mathrm{~min}$. Aliquots ( $4 \mathrm{vol}$.) were then transferred to a polystyrene 96 wells plate (Immulon 2HB, Dynex Technologies), already containing 1 vol. of buffer A (20 $\mathrm{mM}$ Hepes, $140 \mathrm{mM} \mathrm{NaCl}, 0.5 \% \mathrm{BSA}$ and $6 \mathrm{pM}$ tissue factor). Coagulation was started by adding 1 vol. of buffer $B$ (2.5 mM Z-GGR-AMC, $20 \mathrm{mM}$ Hepes, $140 \mathrm{mM} \mathrm{NaCl}, 100 \mathrm{mM} \mathrm{CaCl} 2$ and $6 \% \mathrm{BSA}$ ). First-derivative curves were converted into nanomolar thrombin concentrations using a calibrator for human $\alpha$-thrombin (27). All analyses were in duplicate. 


\section{Statistics}

Differences between groups were tested for significance with the non-parametric MannWhitney $U$ test. Paired data were compared with a Student t-test. The statistical package for social sciences (SPSS 15.0) was used.

\section{Results}

Deficiency in platelet STIM1 or Orai1 impedes GPVI-mediated PS exposure and thrombus formation

For the experiments, chimeric mice were generated by transplantation of irradiated wild type animals with Stim1 ${ }^{-1}$, Orai1 ${ }^{-1-}$ or wild type bone marrow cells. Platelets from these mice, isolated at least 4 weeks after transplantation, were checked for absence of STIM1 protein or Oraiı transcript (supplemental Fig. $1 \mathrm{~A}, \mathrm{~B}$ ). The platelets from chimeric Stim1 ${ }^{-1}$ and Orair $^{-1-}$ mice were greatly impaired in $\mathrm{Ca}^{2+}$ rises evoked by the GPVI agonist, CRP (a triple helical collagen peptide), or by thrombin, most markedly when extracellular $\mathrm{CaCl}_{2}$ was present (supplemental Fig. $1 \mathrm{C}$ ), which is in full agreement with earlier data $(23,24)$. In addition, Stimi ${ }^{-1-}$ platelets showed reduced $\mathrm{Ca}^{2+}$ rises in the absence of $\mathrm{CaCl}_{2}$ and $\mathrm{Ca}^{2+}$ entry, supporting the concept that STIM1 controls the filling state of $\mathrm{Ca}^{2+}$ stores (23).

A

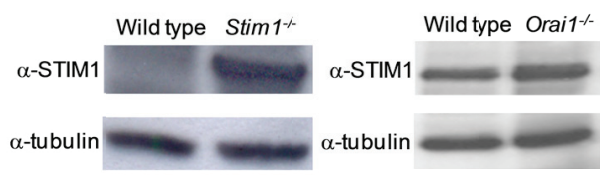

C

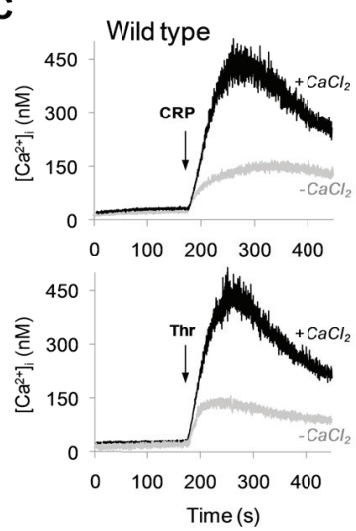

B

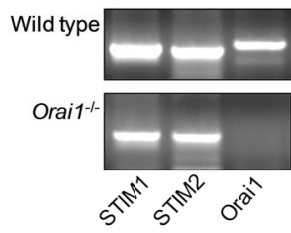

Orai1-1-
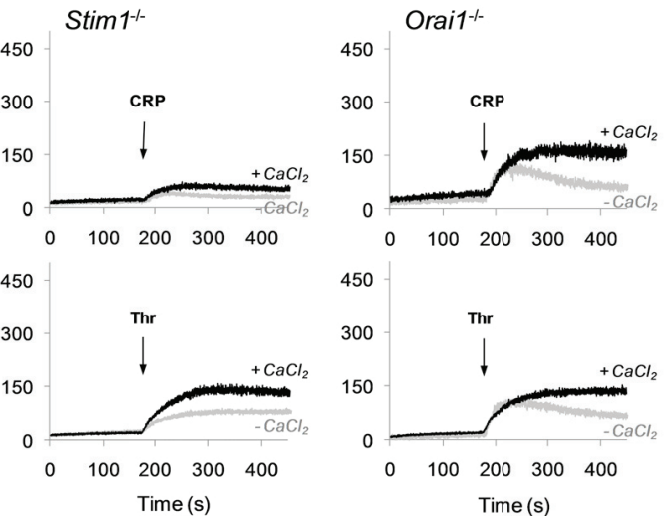

Supplemental figure 1. Defective GPVI- and thrombin-receptor induced $\mathrm{Ca}^{2+}$ signaling in platelets deficient in STIM1 or Orai1. A, Expression profiles of STIM1 protein in platelets from wild type (WT) and chimeric Stim1 ${ }^{-1-}$ or Orai1 ${ }^{-1-}$ mice. Western blots are given after probing with anti-STIM1 mAb; blots were reprobed with anti-tubulin $\mathrm{mAb}$ as control. B, RT-PCR indicating absence of Orai1 transcript but presence of Stim1 and Stim2 transcripts in platelets from chimeric Orai1 ${ }^{-1-}$ mice. C, impaired $\mathrm{Ca}^{2+}$ responses of Stimi ${ }^{-1-}$ and Oraii ${ }^{-1}$ platelets especially in the presence of $\mathrm{CaCl}_{2}$. Fura-2-loaded platelets were stimulated with $10 \mu \mathrm{g} / \mathrm{ml}$ CRP (upper panels) or $0.9 \mathrm{nM}$ thrombin (Thr, lower panels) in the presence or absence of $1 \mathrm{mM} \mathrm{CaCl}$, as indicated. Shown are representative traces of rises in $\left[\mathrm{Ca}^{2+}\right]_{i}(n=3-5)$. 
Blood from these chimeric mice was anticoagulated with PPACK/heparin, and perfused at high-shear flow rate over a collagen surface. By using these thrombin inhibitors, blood coagulation was ablated, while high, physiological concentrations of free $\mathrm{Ca}^{2+}$ and $\mathrm{Mg}^{2+}$ were maintained. In this test of collagen-induced thrombus formation, platelets in contact with the collagen fibers become activated via GPVI, and respond by $\mathrm{Ca}^{2+}$ elevation, PS exposure and secretion of autocrine mediators, with as a result platelet aggregate formation $(4,32,36)$. Strikingly, with blood from chimeric Stim ${ }^{-1-}$ and Orai1 ${ }^{-1-}$ mice, no or only small aggregates were formed, while PS exposure was almost completely absent, as apparent from the lack of staining with FITC-labeled annexin $A_{5}$ (Fig. 1A). This contrasted to the large aggregates and many PS-exposing platelets observed with blood from corresponding wild type mice. Analysis of microscopic images pointed to a markedly reduced deposition of Stim1 $^{-1-}(-70 \%)$ and Orai1 ${ }^{--}(-46 \%)$ platelets in comparison to wild type. The procoagulant index, i.e. the relative formation of PS-exposing platelets compared to all adhered platelets (32), was also greatly reduced in either knockout (Fig. 1B).

Mouse platelets were loaded with Fluo- 4 and back-added to blood of the same genotype in order to measure $\mathrm{Ca}^{2+}$ rises in the cells during blood flow over collagen. Wild type platelets showed high $\mathrm{Ca}^{2+}$ responses shortly after adhesion (Fig. 2A). However, adhered $\mathrm{Stim1}^{-1-}$ platelets gave only minute $\mathrm{Ca}^{2+}$ spikes, in a manner resembling the low responses of Fcer1 $^{-1-}$ platelets, known to lack GPVI signaling activity (33). Many of the adhered Orair ${ }^{-1-}$ platelets gave similar, minute $\mathrm{Ca}^{2+}$ spikes $(\sim 50 \%)$, but the remaining Orai1 ${ }^{-1-}$ cells showed short series of medium amplitude spikes (Fig. 2A). Quantitative analysis demonstrated a nearly complete ablation of the average $\mathrm{Ca}^{2+}$ signal in $\mathrm{Stim}^{-1-}$ platelets and a greatly reduced signal in Orai ${ }^{-1-}$ platelets (Fig. 2B). Together, these results point to important roles of both STIM1 and Orai1 in collagen-dependent $\mathrm{Ca}^{2+}$ signaling, PS exposure and thrombus formation under non-coagulant conditions.

Deficiency in STIM1 or Orai1 does not abolish PS exposure and thrombus formation under coagulant conditions

GPVI-induced platelet activation has been shown to control collagen-dependent thrombus formation also under conditions favoring coagulation (28). To determine the role of STIM1 and Orai1 in this setting, citrate-anticoagulated blood from chimeric animals was co-infused with $\mathrm{CaCl}_{2} / \mathrm{MgCl}_{2}$ to achieve again millimolar free $\mathrm{Ca}^{2+}$ and $\mathrm{Mg}^{2+}$ concentrations. Perfusion of the recalcified blood over collagen leads to onset of coagulation via collagen-dependent activation of factor XII (30). With wild type blood, massive and dense thrombi (clots) were formed within 4 min of perfusion, which were covered with PS-exposing platelets and connected by fibrin fibers (Fig. 3A). Surprisingly, dense fibrin-containing thrombi were also formed with Stim1 ${ }^{-/-}$or Orai ${ }^{-/}$blood. These thrombi were also surrounded by PS-exposing platelets, but to a lesser degree in comparison to wild type blood. However, the procoagulant index was similarly high for images recorded from wild type, Stim1 ${ }^{-1}$ and Orai1 ${ }^{-1}$ thrombi (Fig. 3B). These results thus indicate that neither STIM1 nor Orai1 is essential for collagen-dependent thrombus formation and PS exposure under coagulant conditions. 
A

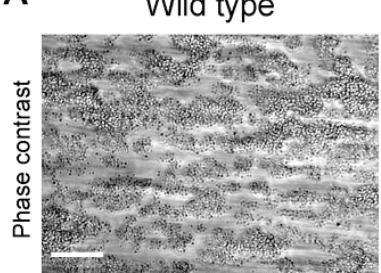

$27.55 \pm 1.08 \%$
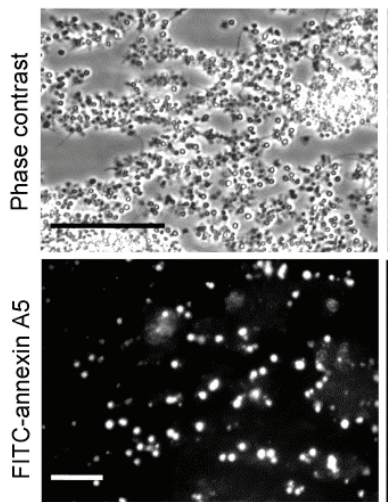

$4.24 \pm 0.22 \%$
Stim $1^{1-}$

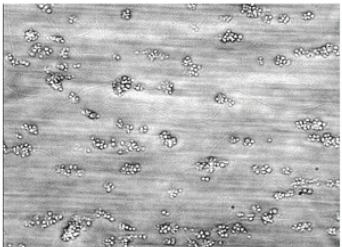

$8.07 \pm 1.78 \%(p<0.01)$
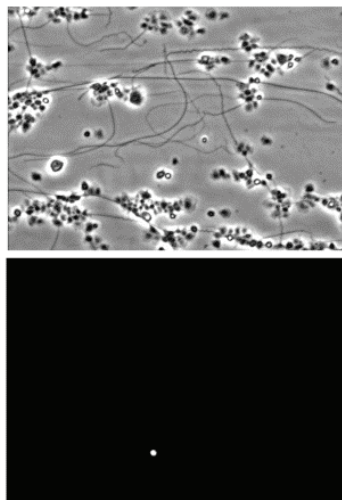

$0.16 \pm 0.05 \%(p<0.01)$
Orai1\%

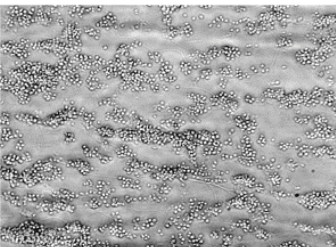

$17.88 \pm 1.24 \%(p<0.01)$

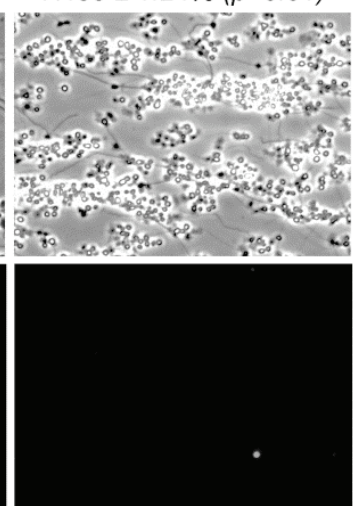

$0.12 \pm 0.03 \%(p<0.001)$

B

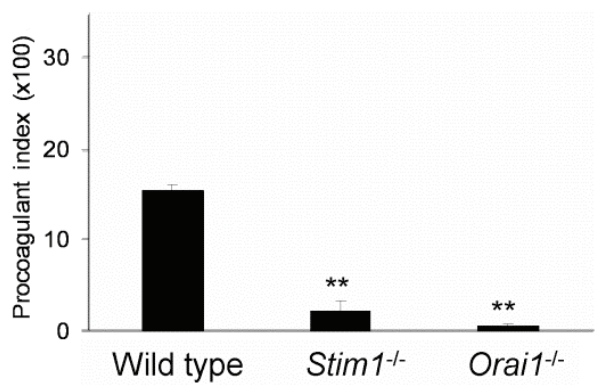

Figure 1. Deficiency in STIM1 or Orai1 impedes GPVI-dependent thrombus formation and PS exposure under flow. PPACK/heparin-anticoagulated blood of $\mathrm{C}_{57} \mathrm{BL} / 6$ mice transplanted with bone marrow from wild type, Stim1 ${ }^{-1-}$ or Orai ${ }^{-1-}$ animals was flowed over collagen at a shear rate of $1000 \mathrm{~s}^{-1}$. $\mathrm{A}$, representative contrast images after $4 \mathrm{~min}$, captured at low (upper panels) or high (middle panels) magnification. Lower panels, fluorescence images after staining with FITC-annexin $\mathrm{A}_{5}$. Percentages in italic indicate area covered with platelets (bars, $50 \mu \mathrm{m}$ ). B, procoagulant index representing relative number of PS-exposing platelets. Data are percentage fractions of adhered platelets exposing PS. Means \pm S.E. $(n=6-8) ; *, p<0.05$ and **, $p<0.01$ versus wild type.

Fluo-4-loaded platelets were then used to determine rises in $\mathrm{Ca}^{2+}$ under the same conditions of flow and coagulation. Wild type platelets, adhered to collagen, showed prolonged, high $\mathrm{Ca}^{2+}$ responses (Fig. $4 \mathrm{~A}$ ), which were higher in level than those measured in anti-coagulated blood (Fig. 2B). With the thrombin inhibitor hirudin present, the mean $\mathrm{Ca}^{2+}$ rises reduced from about 400 to $250 \mathrm{nM}$, which confirmed the contribution of thrombin to 
the $\mathrm{Ca}^{2+}$ signal. Interestingly, in recalcified blood, also collagen-adhered $\mathrm{Stim1}^{-1-}$ and Orai1 ${ }^{-1-}$ platelets showed prolonged $\mathrm{Ca}^{2+}$ responses, but these remained lower in magnitude than those of wild type platelets (Fig. 4B). The effect of STIM1 knockout was again more pronounced than that of Orai1 deficiency. Hence, under these conditions of in situ formation of thrombin, platelet $\mathrm{Ca}^{2+}$ responses seem to be sufficiently high for PS exposure even in the absence of the STIM1-Orai1 SOCE pathway.
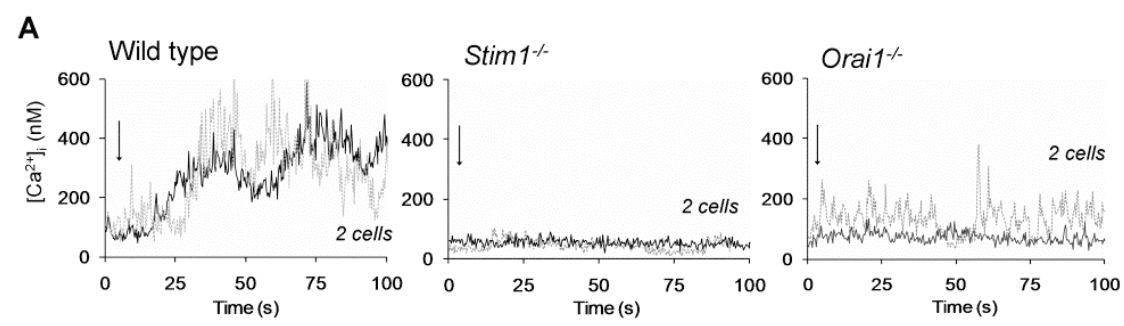

B

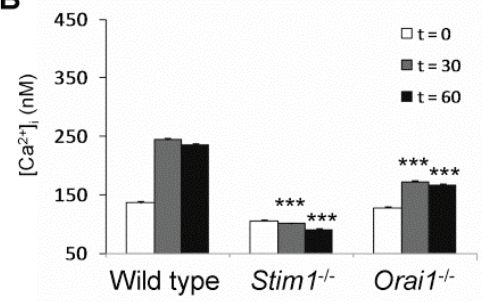

Figure 2. Deficiency in STIM1 or Orai1 impedes GPVI-dependent $\mathrm{Ca}^{2+}$ responses of collagenadhered platelets under flow. PPACK/heparin-anticoagulated blood of wild type or chimeric Stim ${ }^{-1-}$ or $\mathrm{Orai}_{1}{ }^{-1}$ mice was supplemented with $10 \%$ Fluo-4-loaded platelets of the same genotype. Blood samples were flowed over collagen at $1000 \mathrm{~s}^{-1}$, and fluorescence images from the collagen surface were recorded at $5 \mathrm{~Hz}$. A, single-cell rises in $\mathrm{Ca}^{2+}$ of 2 representative platelets per genotype. Arrows indicate time point of adhesion. $\mathrm{B}$, quantitative analysis of $\mathrm{Ca}^{2+}$ responses at 30 and $60 \mathrm{~s}$ after initial $\mathrm{Ca}^{2+}$ rises. Means \pm S.E. $(n=35-45$ cells $) . * * *, p<0.001$ compared to wild type.

Deficiency in STIM1 or Orai1 does not affect GPVI-induced PS exposure and prothrombinase activity with thrombin present

To investigate this further, washed platelets were stimulated with collagen (GPVI)- and/or thrombin-receptor agonist, and staining of the cells was observed with the PS probe, FITCannexin $\mathrm{A}_{5}$. Extracellular $\mathrm{CaCl}_{2}$ was present to allow $\mathrm{Ca}^{2+}$ entry. The GPVI agonist convulxin was used for these studies (12), which is similarly effective as CRP in wild type and Orai1deficient platelets (25). Flow cytometric analysis showed that convulxin stimulation of wild type platelets resulted in about 20\% PS-exposing platelets (Fig. $5 \mathrm{~A}$ ). This fraction increased to $73 \%$ with thrombin as co-agonist, whereas thrombin alone had no more than little effect. Stimulation of platelets from chimeric Stim ${ }^{-1-}$ and Orail $^{-{ }^{--}}$mice with convulxin resulted in impaired PS exposure, comparable to stimulation with thrombin alone. However, combined stimulation with convulxin and thrombin resulted in 53-58\% PS positive platelets, which was similar to that of wild type platelets. Control experiments indicated that all platelets responded nearly completely, when stimulated with the $\mathrm{Ca}^{2+}$ ionophore, ionomycin. As an 
alternative approach, we studied the capacity of platelets to support prothrombinase activity, i.e., the PS-dependent cleavage of prothrombin by factors Xa and Va (12). In wild type platelets, but not Stimi ${ }^{-1-}$ or Orai1 ${ }^{-1-}$ platelets, convulxin caused a moderate increase in prothrombinase activity (Fig. 5B). The combination of convulxin and thrombin induced a fivefold increased prothrombinase activity with wild type platelets and a slightly lower increase with knockout platelets, but this was not significantly different from wild type. Stimulation with ionomycin/ $\mathrm{CaCl} 2$ caused high, maximal prothrombinase activity in all groups.

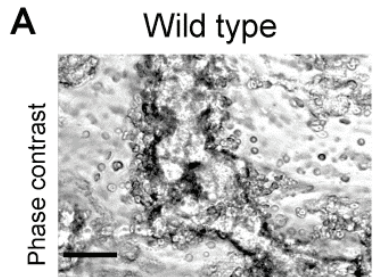

$53.22 \pm 2.34 \%$

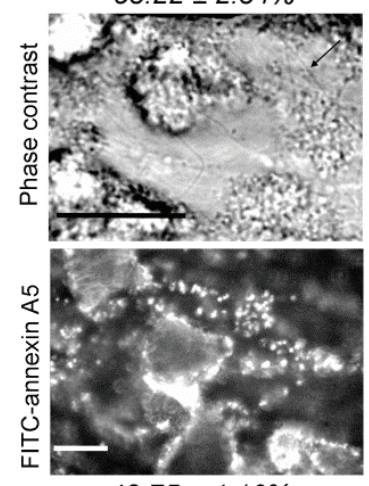

$42.75 \pm 4.10 \%$

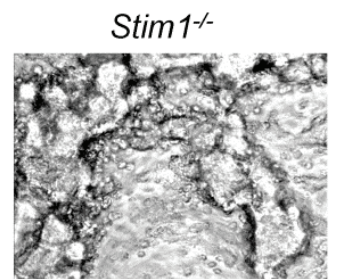

$32.71 \pm 6.80 \% \quad(p=0.068)$
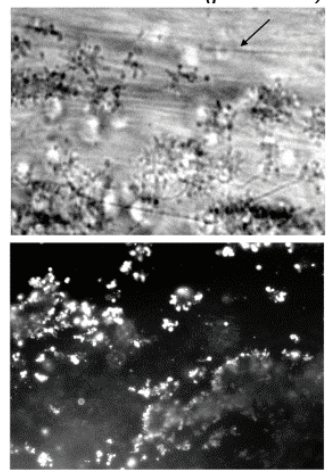

$23.52 \pm 4.42 \% \quad(p<0.05)$
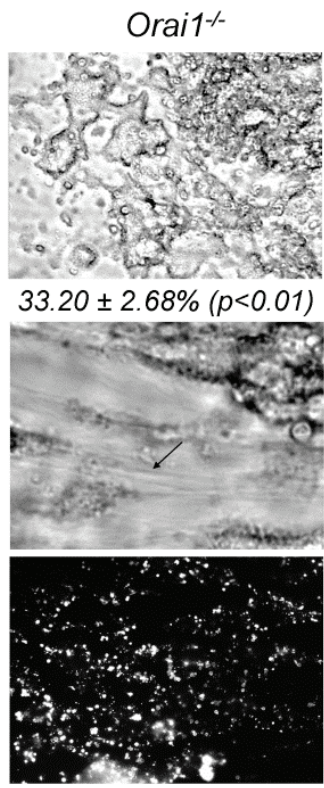

$23.46 \pm 4.57 \%(p<0.05)$

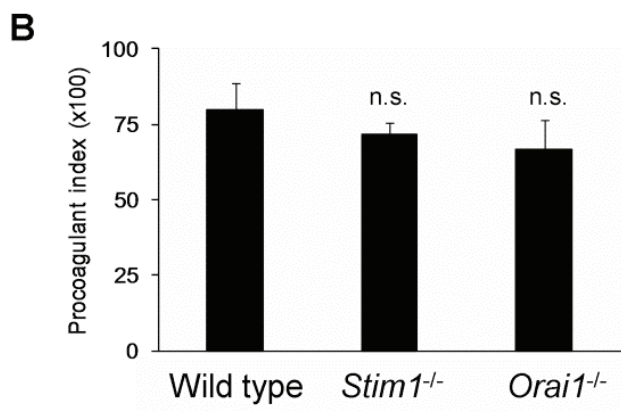

Figure 3. Deficiency in STIM1 or Orai1 permits GPVI-dependent thrombus formation and PS exposure in the presence of coagulation. Citrate-anticoagulated blood of indicated mice was recalcified with $\mathrm{CaCl}_{2} / \mathrm{MgCl}_{2}$ and flowed over collagen (4 min). Thrombi with platelets and fibrin (arrows) were post-stained with FITC-annexin $A_{5}$. A, representative phase contrast and fluorescence images after 4 min (bars, $50 \mu \mathrm{m})$. Percentages in italic indicate area covered with (fluorescent) platelets. B, procoagulant index of relative number of PS-exposing platelets. Means \pm S.E. $(n=5-7)$; difference between groups not significant. 
The procoagulant response of platelets was also studied in natural plasma environment, by activating PRP with tissue factor/ $\mathrm{CaCl} 2$ and measuring generation of thrombin (4). Similar thrombin generation curves were obtained with PRP from wild type, Stim ${ }^{-1}$ and Orai1 $^{-1-}$ mice (Fig. 6A). In all groups, pre-stimulation with ionomycin caused a 3-4 fold increase in thrombin generation, indicating that the knockout platelets were normally capable to support thrombin generation (Fig. 6B). Pre-stimulation with convulxin resulted in a significant increase in thrombin peak height in all groups, but in this case STIM1- or Orai1deficient PRP was less effective than wild type PRP in comparison to pre-stimulation with ionomycin. The time-to-peak was less shortened in convulxin-stimulated knockout PRP in comparison to wild type (Fig. 6C). Taken together, these results show that, in the presence of thrombin (either externally added or formed in situ), GPVI-dependent PS exposure and procoagulant activity are only partly affected by deficiency in platelet STIMı or Oraiı.
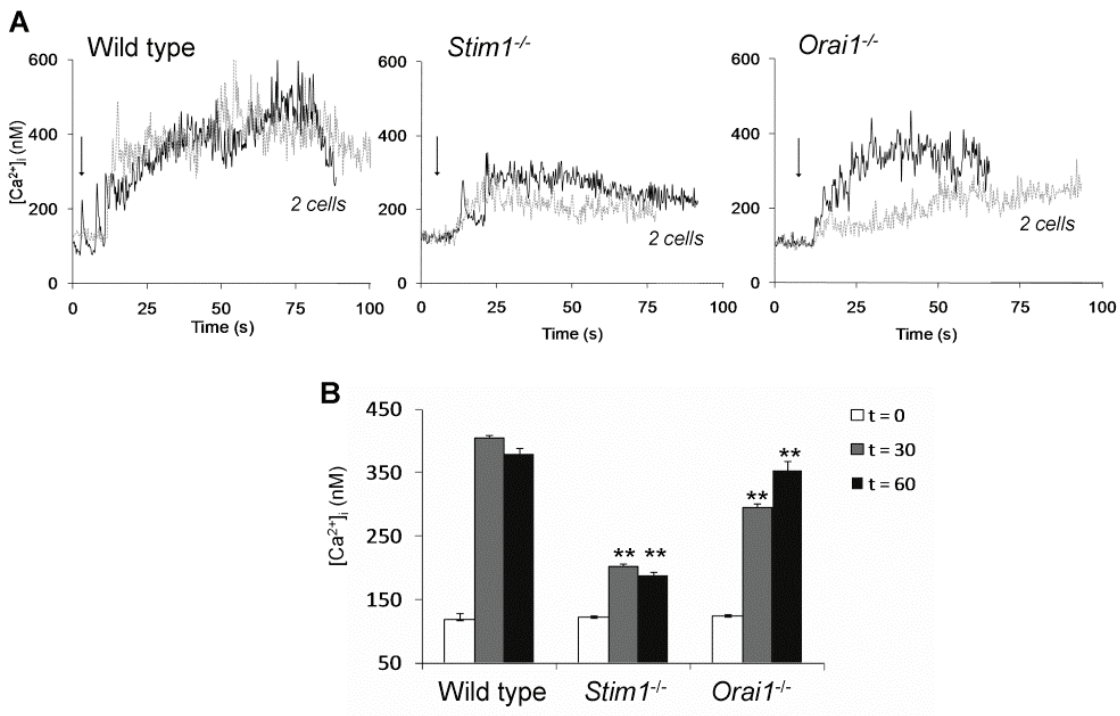

Figure 4. Deficiency in STIM1 or Orai1 partly reduces $\mathrm{Ca}^{2+}$ signaling in the presence of coagulation. Citrate-anticoagulated blood of indicated mice was supplemented with 10\% Fluo-4-loaded platelets of the same genotype, and recalcified. During blood flow over collagen, fluorescence images from the collagen surface were recorded at $5 \mathrm{~Hz}$. A, single-cell rises in $\left[\mathrm{Ca}^{2+}\right]_{i}$ of 2 representative platelets per genotype. Arrows indicate time point of adhesion. B, quantitative analysis of $\mathrm{Ca}^{2+}$ responses at 30 and $60 \mathrm{~s}$ after initial $\left[\mathrm{Ca}^{2+}\right]_{\mathrm{i}}$ rises. Means \pm S.E. $(n=15-29$ cells $) . * *, p<0.01$ compared to wild type.

\section{Roles of $\mathrm{Ca}^{2+}$ entry channels other than Orain}

The modest roles of STIM1 and Orai1 in GPVI-dependent platelet procoagulant activity in the presence of thrombin suggests the presence of a compensating $\mathrm{Ca}^{2+}$ entry mechanism, that for instance could be triggered in a receptor-operated fashion. In other cell types, it has been shown that also STIM2 regulates $\mathrm{Ca}^{2+}$ entry by controlling cytoplasmic $\mathrm{Ca}^{2+}$ levels (37). Since mouse platelets highly express this isoform (supplemental Fig. 1B), we studied the possibility that STIM2-mediated $\mathrm{Ca}^{2+}$ regulation controls the extent of PS exposure. Mice 
homozygously deficient in STIM2 are viable and have normal platelet counts (26). Stimulation of Fura-2-loaded Stim2 ${ }^{-1-}$ platelets showed unaltered $\mathrm{Ca}^{2+}$ responses upon stimulation of GPVI (Fig. 7A, B) or thrombin-receptors (not shown). Flow perfusion experiments over collagen, performed with blood from the Stim2 ${ }^{-1-}$ mice, indicated the formation of large thrombi and the presence of many PS exposing platelets (Fig. 7C). Furthermore, detailed image analysis pointed to an unchanged procoagulant index of Stim2 $2^{-/-}$thrombi in comparison to Stim2 ${ }^{+/}$thrombi (Fig. $\left.7 \mathrm{D}\right)$.

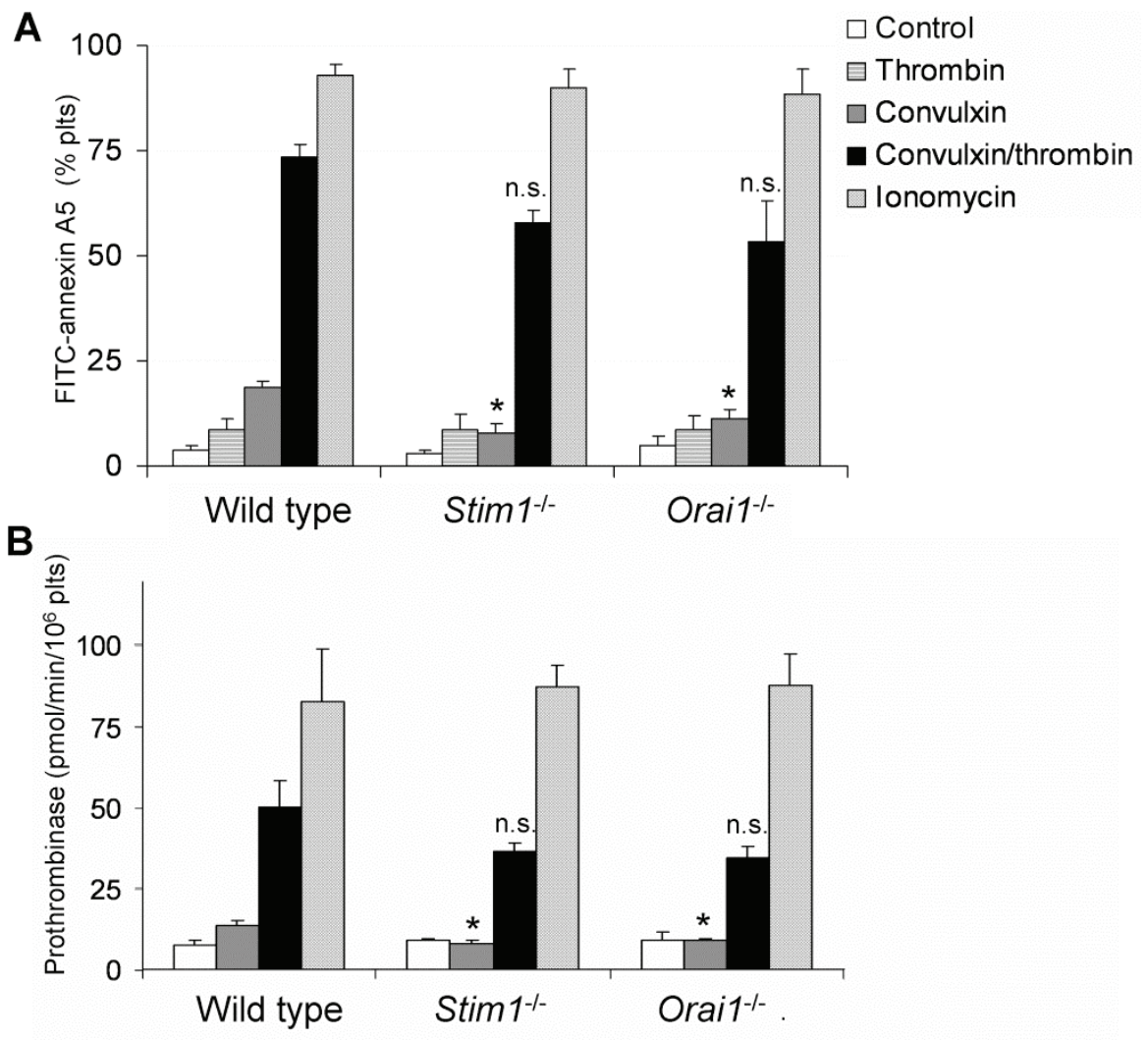

Figure 5. Deficiency in STIM1 or Orai1 permits GPVI-dependent procoagulant activity in the presence of thrombin. Platelets in buffer containing $2 \mathrm{mM} \mathrm{CaCl} 2\left(1 \times 10^{8} / \mathrm{ml}\right)$ were stimulated for $10 \mathrm{~min}$ with convulxin $(100 \mathrm{ng} / \mathrm{ml})$, thrombin $(4 \mathrm{nM})$, or ionomycin $(20 \mu \mathrm{M})$, as indicated. $\mathrm{A}$, flow cytometric analysis of binding of FITC-annexin $A_{5}$ to platelets $(n=5)$. B, determination of prothrombinase activity of platelet suspensions. Activity is expressed as pmol thrombin $/ \mathrm{min} / 10^{6}$ platelets $(n=7-8)$. Means \pm S.E.. $*, p<0.05$ compared to wild type.

The putative role of other $\mathrm{Ca}^{2+}$ entry channels was investigated by using the established $\mathrm{Ca}^{2+}$ entry blocker, SKFg6365 (38). To compare with earlier established $\mathrm{Ca}^{2+}$ traces (supplemental Fig. 1), Fura-2-loaded platelets were activated with GPVI agonist CRP, thrombin or the combination of both. In wild type platelets, pretreatment with SKF96365 
inhibited the $\mathrm{Ca}^{2+}$ responses to all agonists with $60-70 \%$, only if $\mathrm{CaCl}_{2}$ was present in the incubation medium (Fig. 8A). On the other hand, when no $\mathrm{CaCl}_{2}$ was added and $\mathrm{Ca}^{2+}$ entry was prevented, SKF96365 did not have a significant effect (Fig. 8B). Pretreatment with SKF96365 had a similar, 70\% inhibitory effect on the $\mathrm{Ca}^{2+}$ response evoked by convulxin, again only in the presence of $\mathrm{CaCl}_{2}$. Strikingly, in $\mathrm{Stim1}^{-1-}$ and $\mathrm{Orai1}^{-1-}$ platelets, $\mathrm{SKF}_{9}{ }_{3} 65$ still suppressed both the CRP- and thrombin-induced $\mathrm{Ca}^{2+}$ responses by $40-60 \%$, again if extracellular $\mathrm{CaCl}_{2}$ was present (Fig. 8B). For all genotypes, the inhibition by SKF96365 was significant in the presence of $\mathrm{CaCl}_{2}(p<0.001)$, but not without $\mathrm{CaCl}_{2}(p=0.05-0.38)$. The $\mathrm{Stim}_{1}{ }^{-1}$ and $\mathrm{Orai1}^{-1-}$ platelets responded similarly, but the former had a lower residual $\mathrm{Ca}^{2+}$ signal in the presence of $\mathrm{SKF}_{9} 6_{3} 6_{5}$, which is explained by the reduced $\mathrm{Ca}^{2+}$ store content in the these cells. Comparison of time traces of knockout platelets shows that the SKF96365inhibitable $\mathrm{Ca}^{2+}$ signal is a relatively slow component, operating after approximately 10-60 $\mathrm{s}$ (Fig. 8A).
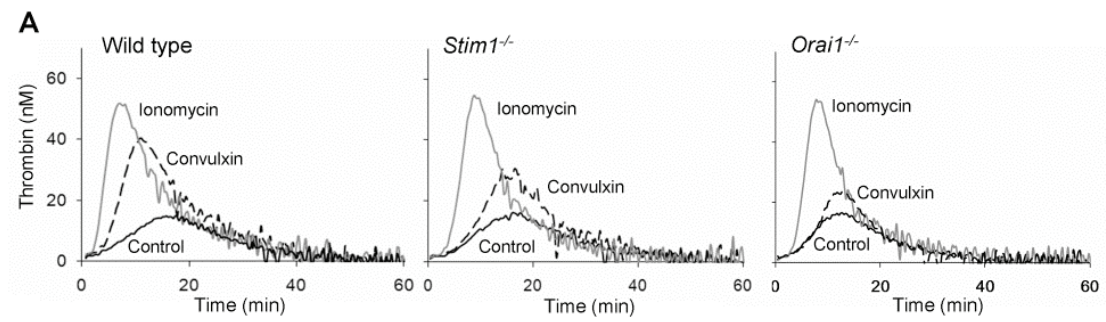

B

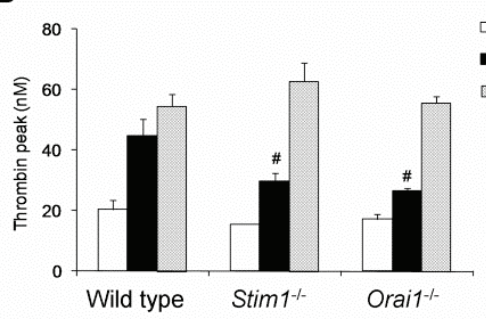

C

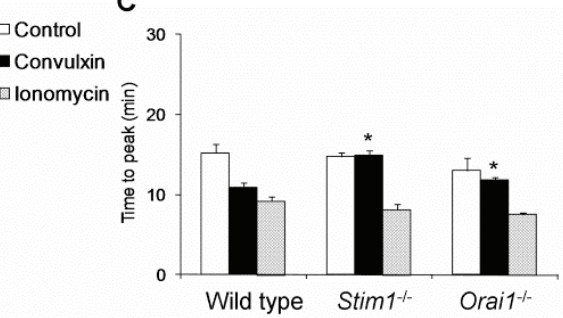

Figure 6. Deficiency in STIM1 or Orai1 reduces GPVI-dependent thrombin generation. Citrateanticoagulated PRP $\left(1 \times 10^{8}\right.$ platelets $\left./ \mathrm{ml}\right)$ of indicated mice was pre-incubated with vehicle solvent (control), convulxin $(100 \mathrm{ng} / \mathrm{ml})$ or ionomycin $(20 \mu \mathrm{M})$. Thrombin generation was triggered with tissue factor/ $\mathrm{CaCl} 2$. A, representative thrombin generation curves per genotype. $\mathrm{B}$, quantification of thrombin peak height. $C$, quantification of time-to-peak. Means \pm S.E. $(n=4-6) . \#, p<0.05$ compared to wild type (ionomycin), *, p $<0.05$ compared to control.

Further experiments demonstrated that the SKF96365-inhibitable $\mathrm{Ca}^{2+}$ entry pathway was important for procoagulant activity, as it suppressed the GPVI/thrombin-mediated PS exposure in both wild type $(-73 \pm 8 \%)$ and Orai $^{-1-}$ platelets $(-34 \pm 6 \%, p<0.05)$. Together, these data point to the involvement of a second $\mathrm{Ca}^{2+}$ entry pathway in platelet PS exposure that is different from the Orai1 channels. 

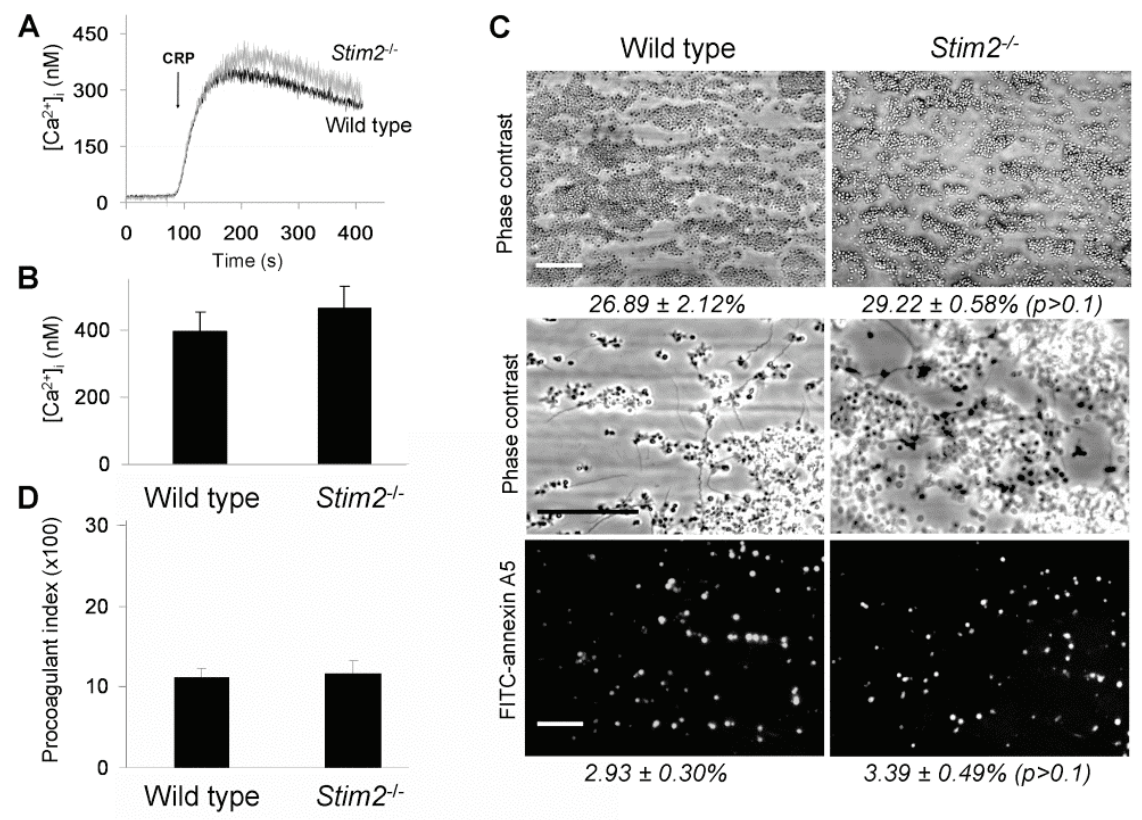

Figure 7. Unchanged GPVI-dependent $\mathrm{Ca}^{2+}$ responses and thrombus formation of STIM2-deficient platelets. A, representative $\mathrm{Ca}^{2+}$ rises of Fura-2-loaded platelets from wild type and $\mathrm{Stim}_{2}{ }^{--}$mice, induced by CRP $(10 \mu \mathrm{g} / \mathrm{ml})$ plus $\mathrm{CaCl}_{2}(1 \mathrm{mM}) ; \mathrm{B}$, quantification of maximal $\mathrm{Ca}^{2+}$ rises. $\mathrm{C}$, D, thrombus formation after flow of PPACK/heparin-anticoagulated blood over collagen at high shear rate $(4 \mathrm{~min})$. C, representative contrast images captured at low (upper panels) or high (middle panels) magnification. Lower panels, fluorescence images after staining with FITC-annexin A5 (bars, $50 \mu \mathrm{m}$ ). D, procoagulant index of relative number of PS-exposing platelets. Means \pm S.E. $(n=4-6)$; difference between groups not significant.

\section{Discussion}

This study examines the contribution of recently discovered $\mathrm{Ca}^{2+}$ entry pathways to the regulation of platelet procoagulant activity and thrombus formation. A first key finding is that deficiency in the $\mathrm{Ca}^{2+}$ sensor STIM1 or the $\mathrm{Ca}^{2+}$-selective channel Orai1 greatly reduces GPVI-dependent PS exposure and thrombus formation under conditions of flow and absence of coagulation. This corresponds to a major reduction in the $\mathrm{Ca}^{2+}$ responses of collagen-adhered Stimi ${ }^{-1-}$ and Orair ${ }^{-1-}$ platelets. Thus, it can be concluded that the reduced GPVIinduced $\mathrm{Ca}^{2+}$ signaling leads to impaired PS exposure and platelet aggregation. Interestingly, platelets from Stim2 ${ }^{-/}$mice are normally active in thrombus formation and PS exposure under the same flow conditions, indicating that the STIM2 homologue is not implicated in these platelet responses. 
A
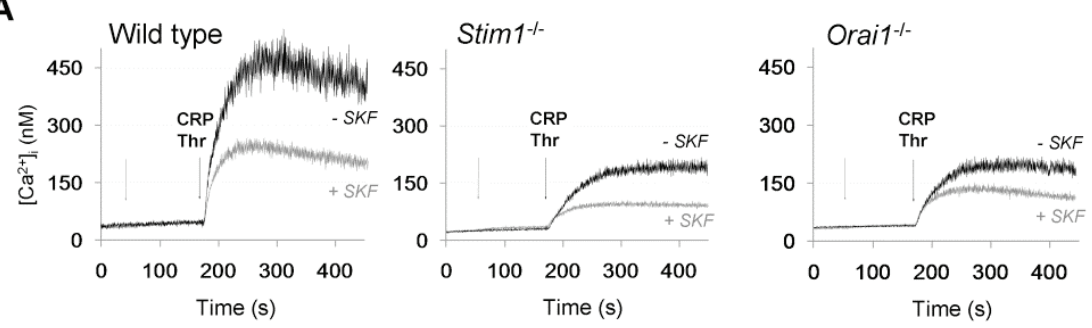

B
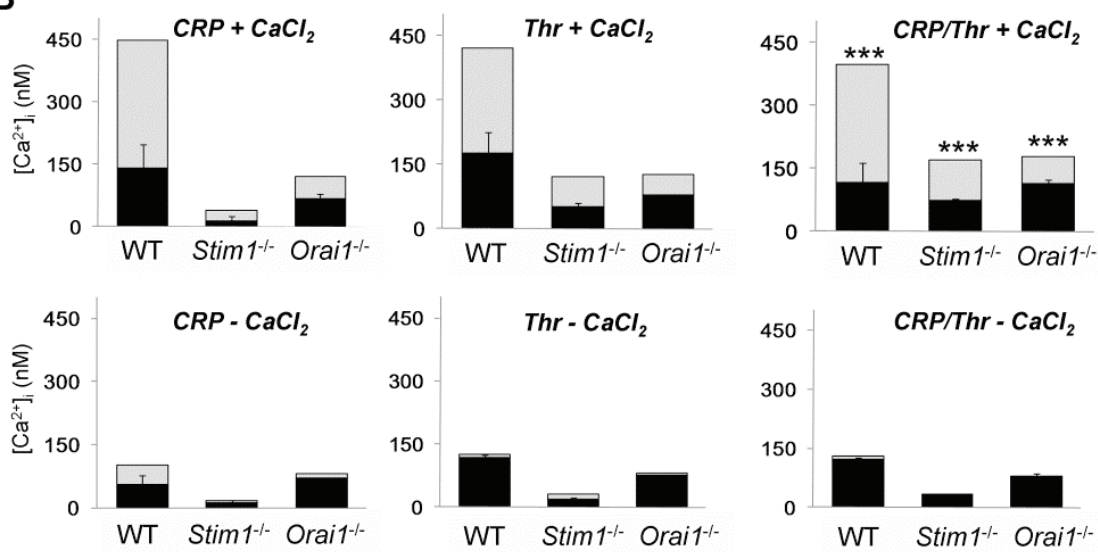

Figure 8. Residual receptor-induced $\mathrm{Ca}^{2+}$ entry in STIM1- and Orai1-deficient platelets. Calcium responses were measured of Fura-2-loaded platelets from wild type (WT), chimeric Stim1 ${ }^{-1}$ or Orai1 ${ }^{-1-}$ mice, induced by CRP $(10 \mu \mathrm{g} / \mathrm{ml})$ and/or thrombin (Thr, $0.9 \mathrm{nM})$ and $\mathrm{CaCl}_{2}(1 \mathrm{mM})$, as indicated. $\mathrm{A}$, representative traces of SKF96365 (SKF, $100 \mu \mathrm{M}$ ) effect on $\mathrm{Ca}^{2+}$ rises. Arrows indicate addition of SKF and agonist(s), respectively. B, quantitative effect of SKF on $\mathrm{Ca}^{2+}$ rises. Total bars give rises without $S K F$, black bars rises with SKF, gray parts represent inhibition by SKF (means \pm S.E., $n=3-4$ ).

Detailed single cell analysis yet points to discernible differences between the genotypes, in that a sub-population of the collagen-adhered Orai1 ${ }^{-1-}$ platelets, but not of the $\mathrm{Stim}_{1}{ }^{-1}$ platelets, displays residual spiking in $\mathrm{Ca}^{2+}$. This residual $\mathrm{Ca}^{2+}$ signal will be a consequence of the appreciable GPVI-induced $\mathrm{Ca}^{2+}$ mobilization from internal stores of Orai1deficient platelet (25), such in contrast to the markedly reduced $\mathrm{Ca}^{2+}$ store mobilization of STIM1-deficient platelets (24). This difference between the genotypes was confirmed in CRP- and thrombin-induced activation studies with Fura-2-loaded platelets. The present findings agree with the recent evidence that a dysfunctional R93W mutation of the Orai1 channel leads to partial impairment of GPVI-induced PS exposure (25). Collectively, our data underline the importance of both SOCE regulating proteins in shear-dependent platelet activation by collagen under conditions where the contribution of thrombin is absent or limited.

The second key finding is that, under conditions of flow and coagulation, deficiency in platelet STIM1 or Orai1 does not abolish the capability to PS exposure and formation of a fibrin-containing thrombus. Under these conditions, collagen-adhered Stim1 ${ }^{-1-}$ and Orair ${ }^{-1-}$ platelets still show prolonged rises in $\mathrm{Ca}^{2+}$, although these are lower in magnitude in com- 
parison to wild type platelets. Although the number of PS exposing Stim1 ${ }^{-1-}$ and $\mathrm{Orai}^{-1-}$ platelets is still reduced, the procoagulant index is similar for all genotypes. This suggests that the GPVI-induced SOCE pathway mediated by STIM 1 and Orai1 becomes redundant in the regulation of thrombus formation in cases where thrombin acts as a co-agonist. This conclusion is supported by the data showing that stimulation of isolated Stim ${ }^{-1-}$ or Orair ${ }^{-1-}$ platelets with a combination of GPVI and thrombin receptor agonists result in near unchanged PS exposure and prothrombinase activity. Furthermore, these platelets are partly inhibited in tissue factor-triggered thrombin generation, when stimulated with GPVI ligand. It has been shown that polyphosphates released from platelets significantly contribute to thrombin generation in PRP by activating factor XII (40). The above findings suggest that the polyphosphate contribution is still effective in the absence of STIM 1 or Orai1.

The standard protocol for assessment of SOCE is measurement of entry of extracellular $\mathrm{Ca}^{2+}$ following $\mathrm{Ca}^{2+}$ store depletion, e.g. by the reticular $\mathrm{Ca}^{2+}$-ATPase inhibitor thapsigargin. This $\mathrm{Ca}^{2+}$ entry is almost totally blocked in Stim ${ }^{-{ }^{-}}$and Orai1 ${ }^{-{ }^{--}}$platelets, implying that the STIM1-Orai1 interaction forms the principal SOCE mechanism $(23,24)$. A similar conclusion has been drawn for other secretory cells, e.g. T cells and mast cells (39). However, it now appears that both Stim ${ }^{-1-}$ and $\mathrm{Orail}^{-{ }^{--}}$platelets show significant $\mathrm{Ca}^{2+}$ responses and PS exposure, when co-stimulated via GPVI and thrombin receptors. This is suggestive for the presence of another, compensatory mechanism of $\mathrm{Ca}^{2+}$ entry, acting in a (thrombin) receptor-operated fashion.

This hypothesis is supported by our finding that the imidazole antagonist SKF96365, which is a well-studied inhibitor of $\mathrm{Ca}^{2+}$ entry in platelets (38), is still capable to suppress the $\mathrm{Ca}^{2+}$ and procoagulant responses of platelets deficient in STIM1 or Orai1. Unfortunately, this compound can not be used in measurements of thrombus formation, since it rapidly inactivates in the presence of blood plasma (data not shown). In Fura-2-loaded Stim1 ${ }^{-1}$ and Oraii $^{-1-}$ platelets, SKF96365 still inhibits $40-60 \%$ of the $\mathrm{Ca}^{2+}$ signal independently of the agonist (GPVI ligand and/or thrombin). The slow, non-Orai1 $\mathrm{Ca}^{2+}$ entry most likely does not involve the TRPC1 channel (15), since Trpc1 ${ }^{-1}$ platelets have a fully intact $\mathrm{Ca}^{2+}$ signaling machinery (40). Other candidate target proteins of SKF96365 are the non-SOCE channels, TRPC6 and Orai3, both of which act in a receptor-operated way, and are expressed in platelets $(16,18)$. Further studies are required to identify this channel.

A pending question is whether the SOCE mechanism per se is implicated in the transmembrane scrambling of phospholipids, which is the underlying event of PS exposure. Such a role of SOCE has been proposed by others (41). However, the current findings do not support this, since Stim1 ${ }^{-1-}$ and Orai1 $^{-1-}$ platelets (both devoid of SOCE) almost normally expose PS upon combined collagen- and thrombin-receptor stimulation. This is in agreement with the finding that platelets from Scott syndrome patients, which have a defect in PS exposure, show unchanged $\mathrm{Ca}^{2+}$ signals in response to collagen/thrombin (42). Together, this strongly argues against a role of SOCE or other $\mathrm{Ca}^{2+}$ channels as phospholipid scramblase proteins.

The present findings support the idea that the Orai1 channel is an attractive target for pharmacological treatment of thrombosis. Previous results have demonstrated that the 
deficiency of Orai1 in mouse platelets protects from arterial thrombus formation, particularly in thrombosis models that are collagen-dependent $(23,24)$. Since collagen-independent thrombosis models mostly rely on tissue factor exposure and thrombin generation (5), it is plausible that specific targeting of Orai1 (or STIM1) restricts platelet procoagulant activity and thrombus formation only under conditions, where the role of tissue factor is limited. Hence, targeting of Oraiı may be more effective to prevent thrombosis in arteries, where (tissue factor-dependent) thrombin accumulation is confined by the high-shear flow conditions. In contrast, blockage of Orai1 may not impair bleeding from wounds where tissue factor is abundantly exposed. Indeed, mice deficient in Orai1 have only a mild prolongation of the tail bleeding time (25). Whether inhibition of the non-Orai1 (non-SOCE) channels targeted by SKF 96365 also provides antithrombotic protection, still needs to be investigated.

\section{Acknowledgements}

We thank Dr. E.M. Bevers for critical discussions and for correcting the manuscript. This work was supported by grants from NWO (11.400.0076), the Netherlands Heart Foundation (2005-B079) and the Deutsche Forschungsgemeinschaft (Sonderforschungsbereich 688 and 487). D.S. acknowledges a grant from the German Excellence Initiative to the University of Würzburg.

\section{References}

1 Heemskerk JW, Bevers EM, \& Lindhout T. Platelet activation and blood coagulation. Thromb Haemost 88, 186-93 (2002).

2 Zwaal RF \& Schroit AJ. Pathophysiological implications of membrane phospholipid asymmetry in blood cells. Blood 89, 1121-32 (1997).

3 Heemskerk JW, Kuijpers MJ, Munnix IC, \& Siljander PR. Platelet collagen receptors and coagulation. A characteristic platelet response as possible target for antithrombotic treatment. Trends Cardiovasc Med 15, 86-92 (2005).

4 Munnix IC, Strehl A, Kuijpers MJ, Auger JM, van der Meijden PE, van Zandvoort MA, oude Egbrink M, Nieswandt B, \& Heemskerk JW. The glycoprotein VI-phospholipase C 22 signaling pathway controls thrombus formation induced by collagen and tissue factor in vitro and in vivo. Arterioscler Thromb Vasc Biol 25, 2673-8 (2005).

5 Kuijpers MJ, Munnix IC, Cosemans JM, van Vlijmen BJ, Reutelingsperger CP, oude Egbrink MG, \& Heemskerk JW. Key role of platelet procoagulant activity in tissue factor- and collagen-

dependent thrombus formation in arterioles and venules in vivo. Differential sensitivity to thrombin inhibition. Microcirculation 15, 269-82 (2008).

6 Schoenwaelder SM, Yuan Y, Josefsson EC, White MJ, Yao Y, Mason KD, O'Reilly LA, Henley KJ, Ono A, Hsiao S, Willcox A, Roberts AW, Huang DC, Salem HH, Kile BT, \& Jackson SP. Two distinct pathways regulate platelet phosphatidylserine exposure and procoagulant function. Blood 114, 663-6 (2009).

7 Léon C, Ravanat C, Freund M, Cazenave JP, \& Gachet C. Differential involvement of the $P_{2} Y_{1}$ and $\mathrm{P}_{2} \mathrm{Y}_{12}$ receptor in platelet procoagulant activity. Arterioscler Thromb Vasc Biol 23, 1941-7 (2003).

8 Van der Meijden PE, Feijge MA, Giesen PL, Huijberts M, van Raak EP, \& Heemskerk JW. Platelet $\mathrm{P}_{2} \mathrm{Y}_{12}$ receptors enhance signalling towards procoagulant activity and thrombin generation. Thromb Haemost 93, 1128-37 (2005).

9 Thiagarajan P \& Tait JF. Collagen-induced exposure of anionic phospholipids in platelets and platelet-derived microparticles. J Biol Chem 266, 24302-7 (1991).

10 Siljander P, Farndale RW, Feijge MA, Comfurius P, Kos S, Bevers EM, \& Heemskerk JW. Platelet adhesion enhances the glycoprotein $\mathrm{VI}$-dependent procoagulant response: Involvement of p38 MAP kinase and calpain. Arterioscler Thromb Vasc Biol 21, 618-27 (2001). 
Nieswandt B \& Watson SP. Platelet-collagen interaction: Is GPVI the central receptor? Blood 102, 449-61 (2003).

12 Bevers EM, Comfurius P, van Rijn JL, Hemker HC, \& Zwaal RF. Generation of prothrombinconverting activity and the exposure of phosphatidylserine at the outer surface of platelets. Eur J Biochem 122, 429-36 (1982).

Dachary-Prigent J, Pasquet JM, Freyssinet JM, \& Nurden AT. Calcium involvement in aminophospholipid exposure and microparticle formation during platelet activation, a study using $\mathrm{Ca}^{2+}$. ATPase inhibitors. Biochemistry 34, 11625-34 (1995).

14 Sage SO. Calcium entry mechanisms in human platelets. Exp Physiol 82, 807-23 (1997).

15 Rosado JA, Brownlow SL, \& Sage SO. Endogenously expressed Trp1 is involved in store-mediated $\mathrm{Ca}^{2+}$ entry by conformational coupling in human platelets. J Biol Chem 277, 42157-63 (2002).

16 Hassock SR, Zhu MX, Trost C, Flockerzi V, \& Authi KS. Expression and role of Trpc proteins in human platelets: Evidence that Trpc6 forms the store-independent calcium entry channel. Blood 100, 2801-11 (2002).

17 Soboloff J, Spassova MA, Tang XD, Hewavitharana T, Xu W, \& Gill DL. Orai1 and Stim reconstitute store-operated calcium channel function. J Biol Chem 281, 20661-5 (2006).

18 Zhang SL, Kozak JA, Jiang W, Yeromin AV, Chen J, Yu Y, Penna A, Shen W, Chi V, \& Cahalan MD. Store-dependent and -independent modes regulating $\mathrm{Ca}^{2+}$ release-activated $\mathrm{Ca}^{2+}$ channel activity of human Orai1 and Orai3. J Biol Chem 283, 17662-71 (2008).

19 Luik RM, Wang B, Prakriya M, Wu M, \& Lewis RS. Oligomerization of STIM1 couples ER calcium depletion to CRAC channel activation. Nature 454, 538-42 (2008).

20 Parvez S, Beck A, Peinelt C, Soboloff J, Lis A, Monteilh-Zoller M, Gill DL, Fleig A, \& Penner R. Stim2 protein mediates distinct store-dependent and store-independent modes of CRAC channel activation. FASEB J 22, 752-61 (2008).

Baba Y, Nishida K, Fujii Y, Hirano T, Hikida M, \& Kurosaki T. Essential function for the calcium sensor STIM1 in mast cell activation and anaphylactic responses. Nat Immunol 9, 81-8 (2008).

22 Oh-hora M, Yamashita M, Hogan PG, Sharma S, Lamperti E, Chung W, Prakriya M, Feske S, \& Rao A. Dual functions for the endoplasmic reticulum calcium sensors STIM1 and STIM 2 in T cell activation and tolerance. Nat Immunol 9, 432-43 (2008).

23 Varga-Szabo D, Braun A, Kleinschnitz C, Bender M, Pleines I, Pham M, Renné T, Stoll G, \& Nieswandt B. The calcium sensor STIM1 is an essential mediator of arterial thrombosis and ischemic brain infarction. J Ex. Med 205, 1583-91 (2008).

24 Braun A, Varga-Szabo D, Kleinschnitz C, Pleines I, Bernder M, Austinat M, Bösi M, Stoll G, \& Nieswandt $B$. Orai $(C R A C M 1)$ is the platelet $S O C$ channel and essential for pathological thrombus formation. Blood 113, 2056-63 (2009).

25 Bergmeier W, Oh-hora M, McCarl CA, Roden RC, Bray PF, \& Feske S. R93W mutation in Orai1 causes impaired calcium influx in platelets. Blood 113, 675-8 (2009).

26 Berna-Erro A, Braun A, Kraft R, Kleinschnitz C, Schuhmann MK, Stegner D, Wultsch T, Eilers J, Meuth SG, Stoll G, \& Nieswandt B. STIM2 regulates capacitive $\mathrm{Ca}^{2+}$ entry in neurons and plays a key role in hypoxic neuronal cell death. Sci Signal 2, ra67 (2009).

27 Vanschoonbeek K, Wouters K, van der Meijden PE, van Gorp PJ, Feijge MA, Herfs M, Schurgers LJ, Hofker MH, de Maat MP, \& Heemskerk JW. Anticoagulant effect of dietary fish oil in hyperlipidemia: An mRNA expression study in APOE2 knock-in mice. Arterioscler Thromb Vasc Biol 28, 2023-9 (2008).

28 Munnix IC, Kuijpers MJ, Auger JM, Thomassen CM, Panizzi P, van Zandvoort MA, Rosing J, Bock $\mathrm{PE}$, Watson SP, \& Heemskerk JW. Segregation of platelet aggregatory and procoagulant microdomains in thrombus formation. Arterioscler Thromb Vasc Biol 27, 2484-90 (2007).

29 Kuijpers MJ, Schulte V, Bergmeier W, Lindhout T, Brakebusch C, Offermanns S, Fässler R, Heemskerk JW, \& Nieswandt B. Complementary roles of glycoprotein VI and $\alpha_{2} \beta_{1}$ integrin in collagen-induced thrombus formation in flowing whole blood ex vivo. FASEB J 17, 685-7 (2003).

30 Van der Meijden PE, Munnix IC, Auger JM, Govers-Riemslag JW, Cosemans JM, Kuijpers MJ, Spronk HM, Watson SP, Renne T, \& Heemskerk JW. Dual role of collagen in factor XII-dependent thrombus formation. Blood 114, 881-90 (2009).

31 Nieswandt B, Brakebusch C, Bergmeier W, Schulte V, Bouvard D, Mohtari-Nejad R, Lindhout T, Heemskerk JW, Zirngibl H, \& Fässler R. Glycoprotein VI but not $\alpha_{2} \beta_{1}$ integrin is essential for platelet interaction with collagen. EMBO J 20, 2120-30 (2001). 
32 Siljander PR, Munnix IC, Smethurst PA, Deckmyn H, Lindhout T, Ouwehand WH, Farndale RW, \& Heemskerk JW. Platelet receptor interplay regulates collagen-induced thrombus formation in flowing human blood. Blood 103, 1333-41 (2004).

33 Auger JM, Kuijpers MJ, Senis YA, Watson SP, \& Heemskerk JW. Adhesion of human and mouse platelets to collagen under shear: A unifying model. FASEB J 19, 825-7 (2005).

34 Heemskerk JW, Willems GM, Rook MB, \& Sage SO. Ragged spiking in free calcium in ADPstimulated platelets: Regulation of puff-like calcium signal in vitro and ex vivo. J Physiol 535, 62535 (2001).

35 Bevers EM, Comfurius P, Reutelingsperger CP, \& Zwaal RF. Platelet procoagulant activity and its measurement. Manuscript, pp. 319-40 (1996).

36 Lecut C, Schoolmeester A, Kuijpers MJ, Broers JL, van Zandvoort MA, Vanhoorelbeke K,

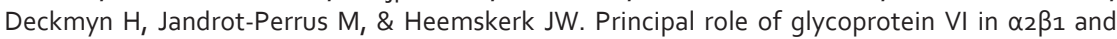
$\alpha \mathrm{llb} \beta 3$ activation during collagen-induced thrombus formation. Arterioscler Thromb Vasc Biol 24, 1727-33 (2004).

37 Brandman O, Liou J, Park WS, \& Meyer T. Stim2 is a feedback regulator that stabilizes basal cytosolic and endoplasmic reticulum $\mathrm{Ca}^{2+}$ levels. Cell 131, 1327-39 (2007).

38 Merritt JE, Armstrong WP, Benham CD, Hallam TJ, Jacob R, Jaxa-Chamiec A, Leigh BK, McCarthy $\mathrm{SA}$, Moores KE, \& Rink TJ. SKF96365, a novel inhibitor of receptor-mediated calcium entry. Biochem J 271, 515-22 (1990).

39 Hewavitharana T, Deng X, Wang Y, Ritchie MF, Girish GV, Soboloff J, \& Gill DL. Location and function of $S T I M 1$ in the activation of $\mathrm{Ca}^{2+}$ entry channels. J Biol Chem 283, 26252-62 (2008).

40 Varga-Szabo D, Authi KS, Braun A, Bender M, Ambily A, Hassock SR, Gudermann T, Dietrich A, \& Nieswandt $\mathrm{B}$. Store-operated $\mathrm{Ca}^{2+}$ entry in platelets occurs independently of transient receptor potential channel 1. Pflugers Arch 457, 377-87 (2008).

41 Martin S, Laude-Lemaire I, Kerbiriou-Nabias D, Freyssinet JM, \& Martinez MC. Relation between phosphatidylserine exposure and store-operated $\mathrm{Ca}^{2+}$ entry in stimulated cells. Biochem Biophys Res Commun 279, 639-45 (2000).

42 Munnix IC, Harmsma M, Giddings JC, Collins PW, Feijge MA, Comfurius P, Heemskerk JW, \& Bevers EM. Store-mediated calcium entry in the regulation of phosphatidylserine exposure in blood cells from Scott patients. Thromb Haemost 89, 687-95 (2003). 



\section{Chapter 7}

Regulation of integrin $\alpha$ llb $\beta 3$ closure in phosphatidyl serine-exposing platelets

Gilio K, Mattheij N, van Kruchten R, Feijge MA, Cosemans JM, Bevers EM, and Heemskerk JW 


\begin{abstract}
Thrombi formed under flow conditions contain microdomains of phosphatidylserine (PS)exposing platelets, separated from aggregated platelets that express activated integrin $\alpha \mathrm{llb} \beta_{3}$. In platelets stimulated with ionomycin or convulxin/thrombin, we aimed to elucidate the mechanism responsible for inactivation or closure of integrin $\alpha$ llb $\beta_{3}$ in PS-exposing platelets. Western blotting indicated that the closure of $\alpha$ llb $\beta_{3}$ coincided with the cleavage of Src protein kinase. However, inhibition of the $\mathrm{Ca}^{2+}$-dependent protease calpain completely antagonized Src cleavage, whereas $\alpha$ llb $\beta_{3}$ closure was only partly reduced. A role of formation of the mitochondrial permeability transition pore (mPTP) was investigated by using the inhibitor cyclosporin A or platelet from mice lacking the MPTP forming protein, cyclophilin D. In either case, convulxin/thrombin-induced $\mathrm{Ca}^{2+}$ rises were no more than moderately affected, whereas integrin closure and PS exposure were more strongly suppressed. Conclusively, this study suggests a model of a calpain-dependent and a mitochondrial (MPTP)-dependent route of integrin closure in PS-exposing platelets.
\end{abstract}

\title{
Introduction
}

Platelet aggregation, in which platelets become connected via active integrin $\alpha \mathrm{llb} \beta_{3}$ and fibrinogen bridges, is fundamental to intravascular thrombus formation $(1,2)$. However, thrombi also contain microdomains of procoagulant platelets, characterized by exposure of phosphatidylserine (PS), which bind coagulation factors and generate thrombin $(3,4)$. In earlier work, the process of PS exposure was shown to rely on a sustained, high intracellular $\mathrm{Ca}^{2+}$ concentration $(3,5)$. PS-exposing platelets assume a balloon-like shape and gradually detach from the thrombus $(1,6)$. Initial experiments suggested that this detachment is linked to inactivation or closure of their $\alpha \mathrm{llb} \beta_{3}$ integrins $(1,7)$.

Glycoprotein VI (GPVI) is the major signaling receptor for collagen on platelets (8). Upon cross-linking of GPVI, the immunoreceptor tyrosine-based activation motif of the FcR $y$-chain undergoes dual tyrosine phosphorylation by members of the Src family kinases, which leads to phosphorylation and activation of the protein tyrosine kinase Syk. This kinase mediates assembly of the LAT signalosome by phosphorylating several docking, adaptor and effector molecules, among which is the key effector enzyme, phospholipase $C_{\gamma 2}$ (PLCY2) $(9,10)$. Activation of PLCY2, further enhanced by phosphoinositide 3-kinases, results in the formation of two second messengers, i.e. 1,2-diacylglycerol and inositol 1,4,5trisphosphate $(11,12)$.

Platelet aggregation relies on the activation of integrin $\alpha$ llb $\beta_{3}$, which provides highaffinity binding sites for fibrinogen (13). Activation of $\alpha$ llb $\beta_{3}$ can be achieved by many platelet agonists via a complex of signaling cascades that is gradually being revealed and includes the small molecule G-protein Rapıb (14-16). One accepted model for the reversible transition from the resting to the activated state of $\alpha$ llb $\beta_{3}$ is by cytoskeletal-mediated unclasping of the $\alpha$ llb and $\beta_{3}$ chains $(17,18)$. Among the proteins involved, linked to the actin cytoskeleton, are filamin A, kindlin-3 and talin-1 $(7,19,20)$.

The process of integrin closure in PS-exposing platelets likely involves more factors than simply a reversion of the activation process (21), but this has not yet been investigated 
in detail. Especially the requirement of sustained and high rises in $\mathrm{Ca}^{2+}$ for agonist-induced PS exposure suggests that different signaling processes are involved in the regulation of $\mathrm{Ca}^{2+}$-dependent integrin closure. There is conflicting evidence for a role of the $\mathrm{Ca}^{2+}$ dependent thiol protease, calpain, in the modulation of $\alpha \mathrm{llb} \beta_{3}$ function. Early studies reported that calpain exerts a positive effect on $\alpha \mathrm{llb} \beta_{3}$ by cleaving talin, enabling talin to bind to the $\beta_{3}$ subunit and provoke unclasping (22). However, other papers considered that calpain activation is a late, $\alpha$ llb $\beta_{3}$-dependent process, mediating proteolysis of signaling and cytoskeletal proteins to achieve full platelet activation $(23,24)$. Another report suggested that activated calpain cleaves the cytoplasmic tail of the $\beta_{3}$ chain, which would counteract the high-affinity conformational state of $\alpha$ llb $\beta_{3}$ (6). Hence, the precise role of calpain in regulating $\alpha$ llb $\beta_{3}$ function is still controversial.

Surface exposure of PS, due to scrambling of phospholipids in the plasma membrane, is provoked by strong $\mathrm{Ca}^{2+}$-mobilizing conditions, such as achieved by $\mathrm{Ca}^{2+}$ ionophores like ionomycin or by specific combinations of platelet agonists, such as convulxin (a GPVI agonist) and thrombin $(3,25)$. A recent study also reports a $\mathrm{Ca}^{2+}$-dependent role of mitochondria in the induction of PS exposure (26). Evidence for this came from the observation that platelets from mice lacking cyclophilin $D(C y p D)$, which is a component of the mitochondrial permeability transition pore (MPTP), are impaired in agonist-induced PS exposure and platelet procoagulant activity $(27,28)$. Whether mPTP formation contributes to the process of integrin closure in PS-exposing platelets is however unknown. Here, we studied the signaling processes involved in the closure of $\alpha \mathrm{llb} \beta_{3}$ in activated, PS-exposing platelets. In particular, we examined the possible roles of Src-family kinases, mPTP formation and calpain-dependent protein cleavage.

\section{Experimental procedures}

\section{Materials}

$\mathrm{H}$-Phe-Pro-Arg chloromethyl ketone (PPACK) and anti-Src mAb (327) were obtained from Calbiochem. Fibrillar type I collagen (Horm) was from Nycomed. Apyrase (grade V), bovine serum albumin (BSA), heparin, and human thrombin were from Sigma. Convulxin was purified to homogeneity from the venom of Crotalus durissus terrificus (Latoxan), as described (29). Fluorescein isothiocyanate (FITC)-labeled annexin A5 was from Nexins Research. Alexa Fluor-647 (AF647)-labeled annexin $A_{5}$, Fura-2 and Fluo-4 acetoxymethyl esters, and pluronic F-127 were from Invitrogen. FITC-labeled PAC1 monoclonal antibody (mAb) against activated human $\alpha \mathrm{llb} \beta_{3}$ came from BD Biosciences. Phycoerythrin (PE)-labeled anti-mouse mAb JON/A was from Emfret Analytics. Anti-phosphotyrosine mAb $4 \mathrm{G} 10$ was from Upstate Biotechnology. Src kinase inhibitors PP2 and Src inhibitor I, Syk inhibitor II, PTP inhibitor I, calpeptin, and cyclosporin A were from Sigma. The PKCO inhibitor (30) was a kind gift from Boehringer Ingelheim Pharmaceuticals. Other materials were obtained from sources indicated before $(31,32)$. 


\section{Animals}

Animal studies were approved by the local animal care and use committees. CypD-deficient mice, which were homozygous for a targeted deletion of the Ppif gene (27), and wildtype $\mathrm{CypD}^{+/+}$mice were generated, as described previously (27), and maintained on an inbred $\mathrm{SV}_{12} 9$ background. Age- and sex-matched mice were used for the experiments.

\section{Blood collection and platelet preparation}

Blood was obtained from healthy volunteers, who had not taken aspirin or related drugs for at least 2 weeks, and gave full informed consent. For whole blood flow experiments, the blood was collected into $1 / 10$ volume saline, containing $40 \mu \mathrm{M}$ PPACK and $40 \mathrm{U} / \mathrm{ml}$ fragmin, as described $(1,33)$. Where indicated, blood was also collected into $1 / 6$ volume ACD solution (85 mM sodium citrate, $78 \mathrm{mM}$ citric acid, and $11 \mathrm{mM}$ D-glucose). Washed platelets were prepared by centrifugation of platelet-rich plasma (PRP) (5), and resuspended into Hepes buffer, pH 7.45 (136 mM NaCl, $10 \mathrm{mM}$ Hepes, $2.7 \mathrm{mM} \mathrm{KCl}, 2 \mathrm{mM} \mathrm{MgCl}$ 2, $0.1 \%$ glucose and $0.1 \%$ BSA). Platelet count was determined with a Coulter counter and adjusted to $1 \times 10^{8}$ platelets $/ \mathrm{ml}$.

Mouse blood was obtained via orbital puncture under anesthesia. The blood was collected into $12.9 \mathrm{mM}$ trisodium citrate, $1 \mathrm{U} / \mathrm{ml}$ heparin, and $5 \mathrm{mM}$ glucose. Washed mouse platelets were prepared from PRP and resuspended in modified Hepes buffer, $\mathrm{pH} 7.45$ (136 $\mathrm{mM} \mathrm{NaCl}, 5 \mathrm{mM}$ Hepes, $2.7 \mathrm{mM} \mathrm{KCl}, 2 \mathrm{mM} \mathrm{MgCl}$, $0.42 \mathrm{mM} \mathrm{Na} 2 \mathrm{HPO}_{4}, 0.1 \%$ glucose, and $0.1 \% \mathrm{BSA}$ ), as described (31).

\section{Platelet flow cytometry and aggregation}

Washed human or mouse platelets $\left(1 \times 10^{8}\right.$ platelets $/ \mathrm{ml}$ ) were preincubated for 10 minutes with $\mathrm{Me} 2 \mathrm{SO}$ vehicle or inhibitor, as indicated in the text. After 5 and 30 minutes of activation with different agonists in the presence of $2 \mathrm{mM} \mathrm{CaCl} 2\left(37^{\circ} \mathrm{C}\right)$, platelet samples were analyzed with a FACScan flow cytometer, equipped with an argon and a red diode laser (Becton Dickinson). Surface expression of PS was detected with AF647-labeled annexin A5 (1:100), while expression of active integrin $\alpha \mathrm{llb} \beta_{3}$ was detected with FITC-labeled PAC1 mAb (1:10) for human platelets, or with PE-labeled JON/A mAb (1:20) for mouse platelets. Aggregation of platelets after activation under stirring conditions was determined by singlecell platelet counting using a Coulter counter (34).

\section{Western blotting and immunohistochemistry}

Platelet samples were lysed in ice-cold NP-40-based lysis buffer pH 7.4 (300 mM NaCl, 20 mM Tris- $\mathrm{HCl}, 2$ mM EGTA, 2 mM EDTA, 2 mM Na3VO 4, 1 mM 4-(2-aminoethyl)benzenesulfonyl fluoride, $10 \mu \mathrm{g} / \mathrm{ml}$ leupeptin, $10 \mu \mathrm{g} / \mathrm{ml}$ aprotinin, $1 \mu \mathrm{g} / \mathrm{ml}$ pepstatin, $2 \% \mathrm{NP}$ 40). Protein amounts in the lysates were quantified with a BioRad DC protein kit. Lysates with equal protein amounts, resolved in $4 \times$ Laemmli sample buffer, were analyzed by $10 \%$ sodium dodecyl sulfate-polyacrylamide gel electrophoresis (SDS-PAGE) and transferred to polyvinylidene difluoride (PVDF) membranes (Millipore), basically as described elsewhere (35). Membranes were immunoblotted using an ECL system (Amersham Biosciences) with 
primary anti-phosphotyrosine $4 \mathrm{G}_{10} \mathrm{mAb}$ or anti-Src mAb, and then stained with horseradish peroxidase-coupled secondary Ab.

\section{Intracellular $\mathrm{Ca}^{2+}$ measurements}

For $\mathrm{Ca}^{2+}$ measurements of human platelets in suspension, PRP $\left(2 \times 10^{8}\right.$ platelets $\left./ \mathrm{ml}\right)$ was loaded with $2.5 \mu \mathrm{M}$ Fura-2 acetoxymethyl ester at $37^{\circ} \mathrm{C}$ for 45 minutes (36). Loaded platelets were isolated, resuspended into Hepes buffer $\mathrm{pH}$ 7.45, and preincubated with indicated inhibitors or $\mathrm{Me} 2 \mathrm{SO}$ vehicle for 10 minutes. Calcium responses were recorded under stirring with a spectrofluorometer (SLM/Aminco) at alternate excitation wavelengths of 340 and $380 \mathrm{~nm}\left(37^{\circ} \mathrm{C}\right)$. Ratio values of fluorescence at $340 / 380 \mathrm{~nm}$ were converted into nanomolar concentrations of $\left[\mathrm{Ca}^{2+}\right]_{\mathrm{i}}$, as described $(36)$. Rises in $\left[\mathrm{Ca}^{2+}\right]_{\mathrm{i}}$ were expressed as 5 minutes time integrals (37).

For single cell $\mathrm{Ca}^{2+}$ measurements in flowing whole mouse blood, washed mouse platelets were incubated with $8 \mu \mathrm{M}$ Fluo-4 acetoxymethyl ester and $0.2 \mathrm{mg} / \mathrm{ml}$ pluronic F127 for 45 minutes at ambient room temperature under gentle rotation (38). The dye-loaded platelets were added to PPACK-anticoagulated blood from the same mouse strain to give $10 \%$ labeled platelets. During the first minute of whole blood perfusion over collagen at a shear rate of $1000 \mathrm{~s}^{-1}$, fluorescence images were captured from the collagen surface at a frequency of $5 \mathrm{~Hz}$. Changes in fluorescence $(F)$ were analyzed in regions-of-interest corresponding to single platelets. Pseudo-ratio F/Fo values were converted into nanomolar concentrations of $\left[\mathrm{Ca}^{2+}\right]_{i}$ using predefined calibration parameters (39).

\section{Statistical analysis}

Differences between experimental groups were tested for significance with the nonparametric Mann-Whitney $U$ test. The statistical package for social sciences (SPSS 17.0) was used.

\section{Results}

Platelet PS exposure and integrin $\alpha / l b b_{3}$ closure coincide with Src kinase cleavage

To reinvestigate the relation between PS exposure and integrin $\alpha$ llb $\beta_{3}$ activation during thrombus formation, both processes were examined in platelet thrombi that were generated by flowing PPACK-anticoagulated whole blood over collagen at intermediately high shear rate of $1000 \mathrm{~s}^{-1}$. As shown in Fig. $1 \mathrm{~A}$, platelet aggregates that were formed on the collagen surface were surrounded by single, bleb-forming platelets, only weakly attached to the aggregates. Dual staining for activated integrin $\alpha$ llb $\beta_{3}$ (using FITC-PAC 1 mAb) and exposure of PS (using AF647-annexin $A_{5}$ ) confirmed the presence of two separate platelet populations, e.g. bleb-shaped, PS-exposing platelets surrounding aggregated platelets with activated integrins. This is in agreement with earlier observations that thrombi are heterogeneous in composition with discrete patches of procoagulant and aggregated platelets (1). Considering that in platelet activation integrin $\alpha$ llb $\beta_{3}$ activation is a fast response and PS exposure is relatively slow, these results suggest that the integrins of PS-exposing platelets are secondarily inactivated. 
In suspensions of human platelets, the ability was studied of activated integrin $\alpha$ llb $\beta_{3}$ to close in time. Two-color flow cytometry was used to examine the binding of FITC-PAC 1 $\mathrm{mAb}$ (detecting activated $\alpha \mathrm{llb} \beta_{3}$ ) as well as AF647-annexin $A_{5}$ (detecting PS exposure). After 5 minutes of activation with convulxin/thrombin in the presence of $\mathrm{CaCl}_{2}$, about $70 \%$ of all platelets bound PAC $1 \mathrm{mAb}$, a fraction which reduced to $25 \%$ at a later time point of 30 minutes after activation (Fig. 1B). On the other hand, the fraction of annexin $\mathrm{A}_{5}$-binding platelets increased between 5 and 30 minutes from $53 \%$ to $78 \%$. Combined analysis of the two colors showed that, after 30 minutes, the vast majority annexin $\mathrm{A}_{5}$-binding platelets did not bind PAC $\mathrm{CAb}_{1}$, while after 5 minutes there was a small sub-population of platelets binding both probes (data not shown). These results thus suggested that convulxin/thrombin stimulation results in a gradual accumulation of PS-exposing platelets, in which the initially activated integrins close in time.

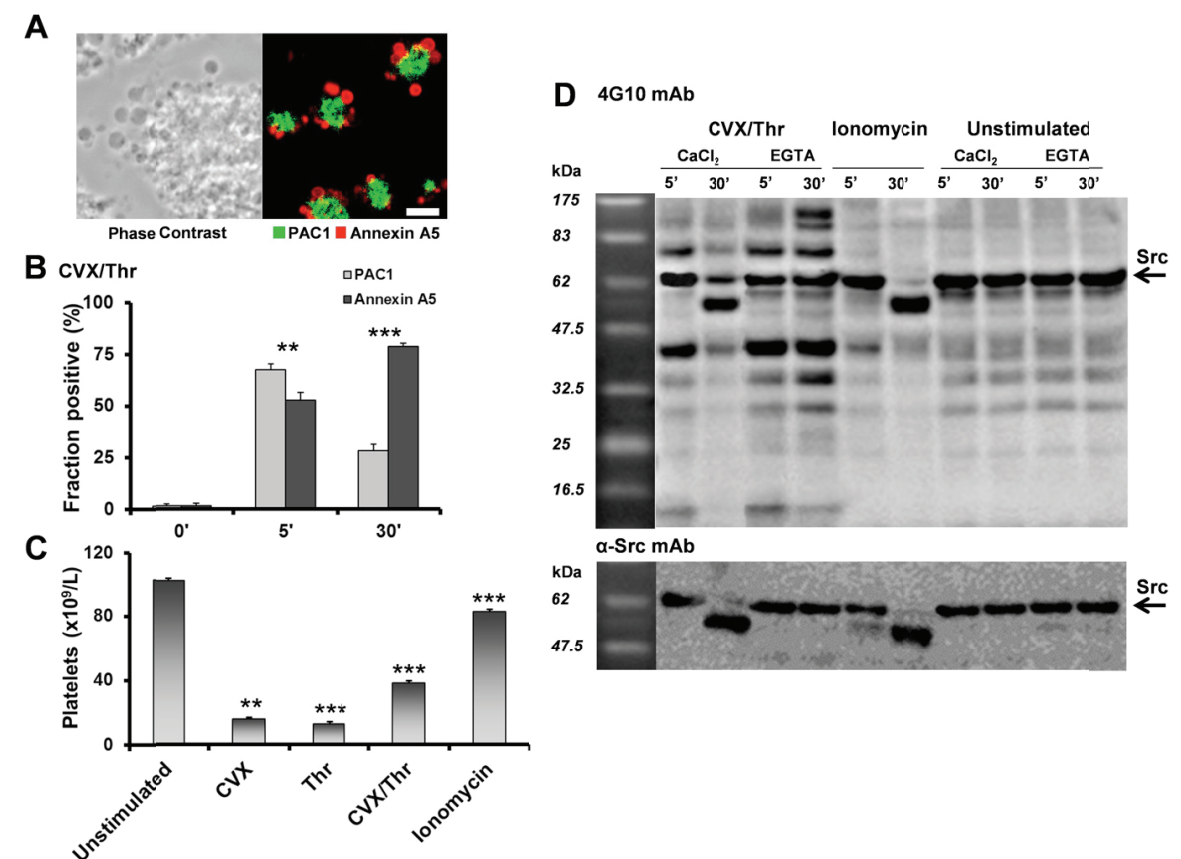

Figure 1. Integrin $\alpha \mathrm{llb} \beta_{3}$ closure in PS-exposing platelets is accompanied by Src kinase cleavage. A, Ex vivo flow experiments were performed with PPACK-anticoagulated human blood perfused over a collagen surface at $1000 \mathrm{~s}^{-1}$ for 4 minutes. Shown is a representative phase contrast image and a twocolor fluorescence image, after staining for activated integrin $\alpha$ llb $\beta_{3}$ (FITC-PAC1 mAb) and PS exposure (AF647-annexin A5); bar, $15 \mu \mathrm{m}$. B, Flow cytometric analysis of washed human platelets stimulated for 5-30 minutes with convulxin $(\mathrm{CVX}, 100 \mathrm{ng} / \mathrm{ml})$ plus thrombin $(\mathrm{Thr}, 4 \mathrm{nM})$ in the presence of $2 \mathrm{mM} \mathrm{CaCl} 2$. Shown are platelet fractions with activated $\alpha$ llb $\beta_{3}$ (FITC-PAC 1 positive) or exposed PS (FITC-annexin A5-positive). Mean \pm S.E. $(n=8-12) ; * *, p<0.02 ; * * *, p<0.001$. C, Single platelet counts after 5 minutes stimulation of platelets with CVX $(100 \mathrm{ng} / \mathrm{ml})$, Thr $(4 \mathrm{nM})$, or ionomycin $(20 \mu \mathrm{M})$, in the presence of 2 $\mathrm{mM} \mathrm{CaCl}$. Mean \pm S.E. $(n=3) ; *, p<0.05$. D, Representative western blots from platelets stimulated for 5-30 minutes with CVX $(100 \mathrm{ng} / \mathrm{ml})$ and Thr $(4 \mathrm{nM})$ or ionomycin $(20 \mu \mathrm{M})$. Activations were in the presence of $2 \mathrm{mM} \mathrm{CaCl} 2$ or a $2 \mathrm{mM} \mathrm{EGTA}$, as indicated. Blots were immunostained with $4 \mathrm{G} 10 \mathrm{mAb}$ (upper), and reprobed with anti-Src Ab (lower panel). Arrows indicate position of Src family proteins. For color version, see page 190. 
To find out whether the gradual integrin closure impairs the ability of platelets to form aggregates, we measured platelet counts in suspension at fixed time points after activation. Previously, this method of single platelet counting was found to be a sensitive way of detecting platelet (micro)aggregates (34). After 30 minutes of activation with either convulxin or thrombin, single platelet counts were reduced to $<15 \%$ of the original count, compatible with persistent, strong platelet aggregation (Fig. $1 \mathrm{C}$ ). In contrast, after 30 minutes activation with convulxin/thrombin, platelet count reduced to only $38 \%$ of the initial count, pointing to an increased number of platelets that was not captured into aggregates. This was confirmed by stimulation with the $\mathrm{Ca}^{2+}$ ionophore ionomycin, causing PS exposure in $>90 \%$ of the platelets, which resulted in only a minor reduction in original platelet count, indicating an absence of platelet aggregation.

To determine the intracellular signaling events of PS-exposing platelets, the protein tyrosine phosphorylation state was examined by staining western blots with $4 \mathrm{G}_{10} \mathrm{mAb}$. Platelets were stimulated during 5-30 minutes with convulxin/thrombin or ionomycin in the presence of $\mathrm{CaCl}_{2}$ or EGTA. With $\mathrm{CaCl}_{2}$ present in the extracellular medium the influx of $\mathrm{Ca}^{2+}$ is known to enhance PS exposure (40). As shown in Fig. $1 \mathrm{D}$, the tyrosine phosphorylation pattern markedly changed in time, under activated conditions where $\mathrm{CaCl}_{2}$ was present. Most striking was a shift of the $60 \mathrm{kDa}$ band present in unstimulated platelets to a band of around $55 \mathrm{kDa}$ after 30 minutes of stimulation with convulxin/thrombin (partly) or ionomycin (completely). Reprobing of the blots with anti-Src mAb indicated that Src kinase was present in the shifted tyrosine-phosphorylated band. Considering that the abundantly expressed Src is tyrosine-phosphorylated in resting platelets (41), these results suggested that Src kinase is cleaved after prolonged platelet activation with convulxin/thrombin or ionomycin, i.e. under activation conditions where platelets expose PS and integrin $\alpha \mathrm{llb} \beta_{3}$ is closed.

Inhibition of Src kinase does not affect integrin closure or PS exposure in activated platelets Whether Src activity is required for the process of integrin closure in PS-exposing platelets was studied with several kinase inhibitors, applied at maximally effective concentrations. We used the compound PP2 $(40 \mu \mathrm{M})$, which is a general Src family kinase inhibitor, and Src inhibitor I $(10 \mu \mathrm{M})$, which only inhibits Src itself $(42,43)$. Integrin closure and PS exposure were simultaneously examined by two color flow cytometry. In the interval between 5 and 30 minutes after platelet activation with convulxin/thrombin, integrin closure and PS exposure were reduced by PP2, but not by Src inhibitor I (Fig. 2A). This may be due to the fact that PP2 suppresses platelet activation via GPVI and PLC 2 , which pathway relies on the Src-family kinase members, Fyn and Lyn (44). Src kinase itself is not involved in this pathway. This was investigated in more detail. 

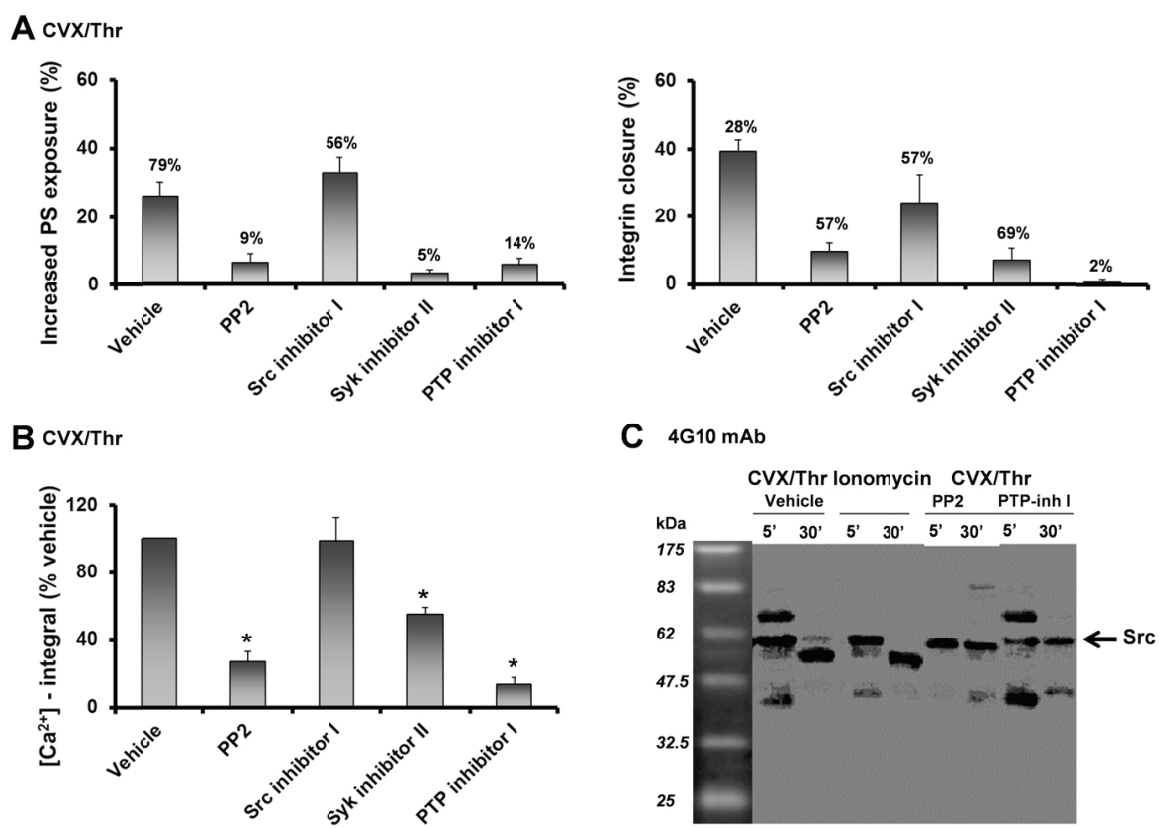

Figure 2. Src kinase inhibition does not affect integrin $\alpha$ llb $\beta_{3}$ closure in PS-exposing platelets. $A$, Increase in PS exposure (left) or integrin closure (right) of human platelets stimulated with convulxin $(\mathrm{CVX}, 100 \mathrm{ng} / \mathrm{ml})$ and thrombin $(\mathrm{Thr}, 4 \mathrm{nM})$ in the presence of $2 \mathrm{mM} \mathrm{CaCl}_{2}$ for 5 to 30 minutes. Platelets were preincubated with vehicle (0.2\% Me2SO), PP2 (40 $\mu \mathrm{M})$, Src inhibitor I (10 $\mu \mathrm{M})$, Syk inhibitor II (1 $\mu \mathrm{M})$ or PTP inhibitor I $(100 \mu \mathrm{M})$, as indicated. Samples were analyzed by dual color flow cytometry for binding of FITC-PAC 1 mAb and AF647-annexin A5. Bars indicate changes between 5 and 30 minutes; numbers above bars indicate absolute percentages of platelets with PS exposure or integrin activation after 30 minutes. Means $\pm \mathrm{S}$.E. $(\mathrm{n}=3)$. B, $\mathrm{Ca}^{2+}$ responses in Fura-2-loaded platelets stimulated with $\mathrm{CVX}$ $(100 \mathrm{ng} / \mathrm{ml})$ and $\mathrm{Thr}(4 \mathrm{nM})$ in the presence of $2 \mathrm{mM} \mathrm{CaCl} 2$. Inhibitors were present as described above. Shown are 5 minutes time integrals of $\mathrm{nM}$ rises in $\left[\mathrm{Ca}^{2+}\right]_{\mathrm{i}}$, expressed as percentages of control $(574 \pm 31$ $\mu \mathrm{M} / \mathrm{min})$. Mean \pm S.E. $(n=3) ; *, p<0.05$. C, Effects of inhibitors on protein tyrosine phosphorylation after 5-30 minutes stimulation with CVX $(100 \mathrm{ng} / \mathrm{ml})$ plus Thr $(4 \mathrm{nM})$ or ionomycin $(20 \mu \mathrm{M})$ in the presence of $2 \mathrm{mM} \mathrm{CaCl}$. Western blots were immunostained with $4 \mathrm{G}_{10} \mathrm{mAb}$. Blot shown is representative for 3 independent experiments. Arrow indicates position of Src family proteins.

First, platelets were preincubated with Syk inhibitor II, which compound blocks Syk kinase (9), and stimulated with convulxin/thrombin. This inhibitor allowed integrins to open, but abolished integrin closure as well as PS exposure in time (Fig. 2A). Second, the GPVI pathway was blocked with protein tyrosine phosphatase (PTP) inhibitor I, which suppresses the phosphatases $\mathrm{SHP}_{1}(45)$ and $\mathrm{PTP}_{1} \mathrm{~B}$ (46). In the presence of PTP inhibitor I, convulxin/thrombin stimulation did not activate platelet integrins (which thus could not close), and greatly suppressed PS exposure in time. Effects of these inhibitors were also measured on convulxin/thrombin-induced rises in intracellular $\mathrm{Ca}^{2+}$ level. Consistent with suppression of the GPVI/PLCY2 pathway, $\mathrm{Ca}^{2+}$ responses were markedly decreased in the presence of $\mathrm{PP}_{2}$, Syk inhibitor II or PTP inhibitor I, but not with Src inhibitor I (Fig. 2B). Western blot analysis indicated that both PP2 and PTP inhibitor I markedly prevented the tyrosine phosphorylated band shift (Fig. $2 \mathrm{C}$ ). Together, these results point to a close association of convulx- 
in/thrombin-induced integrin closure and PS exposure, relying on GPVI stimulation and high rises in $\mathrm{Ca}^{2+}$ and associated with Src degradation. However, the failure of Src inhibitor I to suppress integrin closure suggests that activation of Src kinase itself is not crucial for this event.
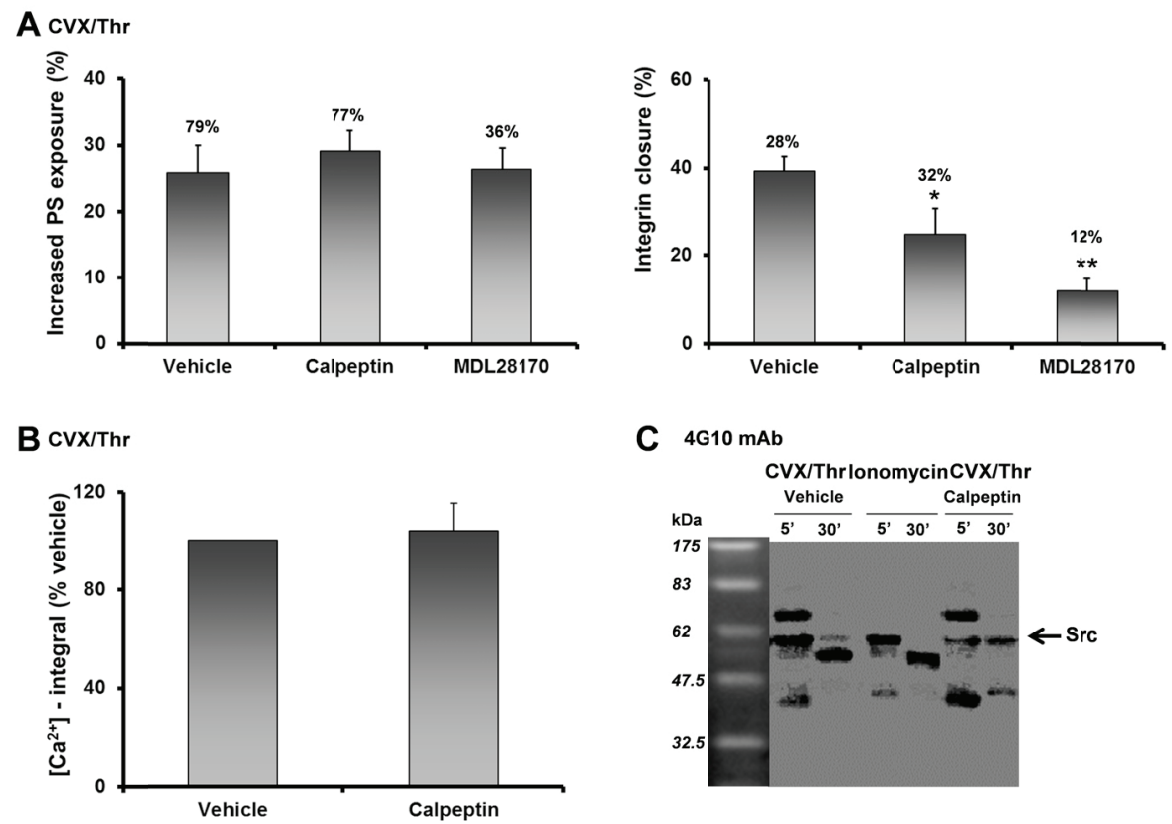

\section{C $4 \mathrm{G} 10 \mathrm{mAb}$}

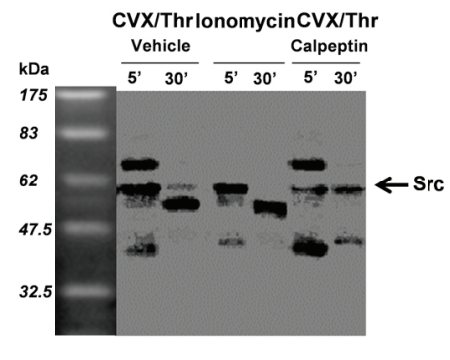

Figure 3. Inhibition of calpain suppresses integrin $\alpha \mathrm{llb} \beta_{3}$ closure and prevents Src kinase cleavage. A, Washed human platelets were stimulated with convulxin $(\mathrm{CVX}, 100 \mathrm{ng} / \mathrm{ml})$ and thrombin $(T h r, 4 \mathrm{nM})$ in the presence of $2 \mathrm{mM} \mathrm{CaCl}$. Platelets were preincubated with vehicle (0.2\% Me2SO), calpeptin (200 $\mu \mathrm{M})$, or MDL28170 $(200 \mu \mathrm{M})$. Time-dependent (5-30 minutes) changes in binding of AF647-annexin $\mathrm{A}_{5}$ (left panel) and FITC-PAC 1 mAb (right panel) were measured by dual color flow cytometry. Numbers above bars represent absolute percentages of platelets with PS exposure or integrin activation, after 30 minutes. $\mathrm{B}$, Intracellular rises in $\left[\mathrm{Ca}^{2+}\right]_{i}$ measured in suspensions of Fura-2-loaded platelets stimulated with CVX $(100 \mathrm{ng} / \mathrm{ml})$ and $\mathrm{Thr}(4 \mathrm{nM})$ in the presence of $2 \mathrm{mM} \mathrm{CaCl}$. Preincubation with vehicle or calpeptin $(200 \mu \mathrm{M})$ was as indicated. C, Western blot and immunostaining with $4 \mathrm{G}_{10} \mathrm{mAb}$ of platelet lysates after preincubation with vehicle or calpeptin $(200 \mu \mathrm{M})$, and time-dependent co-stimulation with CVX $(100 \mathrm{ng} / \mathrm{ml})$ plus $\operatorname{Thr}(4 \mathrm{nM})$ or ionomycin $(20 \mu \mathrm{M})$ in the presence of $2 \mathrm{mM} \mathrm{CaCl}$. Means $\pm \mathrm{S}$.E. $(n=3) ; *, p<0.05, * *, p<0.02$ compared to vehicle. Arrow indicates position of Src family proteins.

\section{Calpain activity contributes to integrin $\alpha_{l l l b b_{3}}$ closure}

Given that the $\mathrm{Ca}^{2+}$-dependent protease calpain can degrade the actin cytoskeleton in PSexposing platelets (47), calpain activation might play a role in integrin closure and Src degradation. To study this hypothesis, the calpain inhibitors calpeptin and MDL28170 were used at a maximally effective concentration of $200 \mu \mathrm{M}(48,49)$. Previously, calpain inhibition has been shown to prevent degradation of the cytoskeleton-associated protein talin (48). Two-color flow cytometry demonstrated that, in convulxin/thrombin-stimulated platelets, pretreatment with calpeptin or MDL28170 partly decreased integrin closure in the time 
interval of 5-30 minutes, while PS exposure was not significantly changed (Fig. 3A). Measurements with Fura-2-loaded platelets indicated that calpeptin did not alter the convulxin/thrombin-induced $\mathrm{Ca}^{2+}$ rises (Fig. ${ }_{3} \mathrm{~B}$ ). In contrast, calpeptin did abolish the Src kinase shift on tyrosine phosphorylation blots (Fig. ${ }_{3}$ C). Together, this denotes a major role of calpain in degradation of Src kinase, but only a partial role in integrin closure.

Subtle role of mitochondrial permeability transition pore (MPTP) formation in integrin $\alpha / l b b_{3}$ closure

Mitochondria act as $\mathrm{Ca}^{2+}$-buffering organelles (50), but this role is disrupted upon mPTP formation (51). Previous work from other laboratories has shown that PS exposure in mouse platelets deficient in cyclophilin $\mathrm{D}(\mathrm{CypD})$ is abolished due to their inability to $\mathrm{MPTP}$ formation $(27,28)$. To investigate the role of MPTP formation in integrin closure, we used the established mPTP inhibitor, cyclosporin A. In human platelets pretreated with cyclosporin A, stimulation with convulxin/thrombin greatly reduced early (5 minutes) PS exposure from $53 \%$ to $4 \%$ and late (30 minutes) PS exposure from $79 \%$ to $9 \%$, (Fig. $4 \mathrm{~A}$ ). Strikingly, this pretreatment also prevented integrin closure in this time interval, but it did not affect integrin activation, as $85 \%$ of the platelets showed activated integrins after 30 minutes. Other experiments showed that cyclosporin A did not influence convulxin/thrombin-induced $\mathrm{Ca}^{2+}$ rises measured in platelet suspension (Fig. 4B). Western blots showed that cyclosporin A did prevent the band shift of tyrosine phosphorylated Src kinase (Fig. $4 \mathrm{C}$ ).

The role of MPTP formation was also examined in pilot studies with platelets from mice homozygously deficient in the protein CypD, which is an essential component of the mPTP. Two-color flow cytometry showed that stimulation of $\mathrm{CypD}^{+/+}$or $\mathrm{CypD}^{-/-}$platelets with ionomycin for 5 minutes resulted in maximal PS exposure ( $94 \pm 1.7 \%$ vs. $91 \pm 2.7 \%)$ and in low integrin activation ( $27 \pm 6.4 \%$ vs. $29 \pm 5.7 \%$ ). This response remained unchanged during the following 25 minutes. Stimulation with convulxin/thrombin ( 5 minutes) led to more integrin activation and less PS exposure in $\mathrm{CypD}^{-/-}$platelets in comparison to wildtypes (Fig. $5 \mathrm{~A})$. The knockout platelets had a similar activation profile after 30 minutes. Pretreatment with cyclosporin $A$ suppressed integrin closure and PS exposure in $\mathrm{CypD}^{+/+}$platelets but not $\mathrm{CypD}^{-1}$ platelets, thus confirming the specificity of cyclosporin A as an inhibitor of MPTP formation. Interestingly, pretreatment with an inhibitor of protein kinase $C \theta$ (PKC $\theta$ ) tended to decrease integrin closure in $\mathrm{CypD}^{+/+}$platelets, but not in $\mathrm{CypD}^{-/-}$platelets. Calpain inhibition with calpeptin gave inconclusive effects. Measurements of $\mathrm{Ca}^{2+}$ rises in Fluo-4-loaded platelets during flow demonstrated a moderate, but significant lowering in $\mathrm{CypD}^{-1} \mathrm{com}$ pared to $\mathrm{CypD}^{+/+}$platelets, especially at early time points (Fig. ${ }_{5} \mathrm{~B}$ ). Together, these results point to a (subtle) role of MPTP formation in integrin closure along with PS exposure in human and mouse platelets. 


\section{A cvx/Thr}
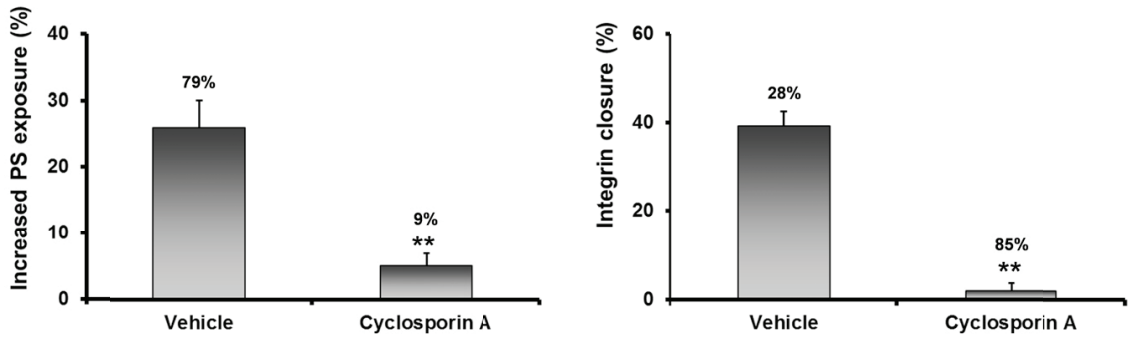

\section{B cvX/Thr}

\section{4G10 mAb}
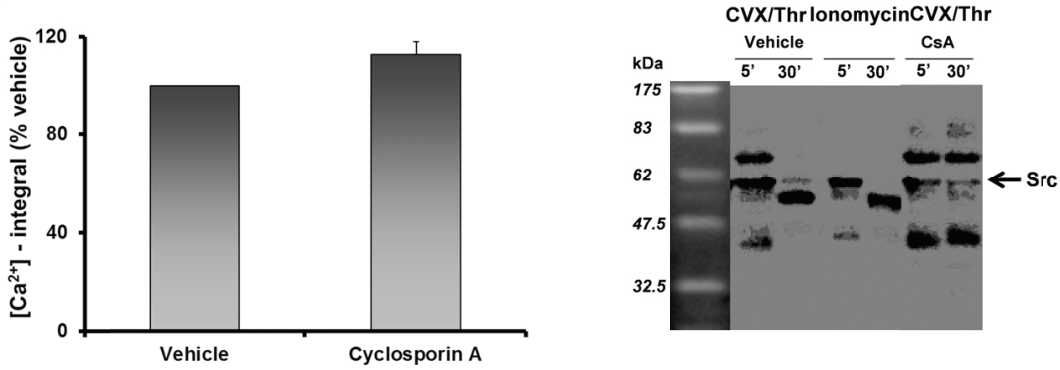

Figure 4. Inhibition of mPTP formation suppresses integrin $\alpha \mathrm{llb} \beta_{3}$ closure and prevents Src kinase cleavage. Washed human platelets were co-stimulated with convulxin (CVX, 100 ng/ml) and thrombin (Thr, $4 \mathrm{nM}$ ) in the presence of $2 \mathrm{mM} \mathrm{CaCl}$ 2. Platelets were preincubated with vehicle (0.2\% Me2SO) or cyclosporin A $(4 \mu \mathrm{M})$. A, Time-dependent changes in binding of AF647-annexin A5 (left panel) and FITCPAC 1 mAb (right panel) was measured by dual color flow cytometry. Numbers above bars indicate absolute percentages of platelets with PS exposure or integrin activation after 30 minutes. B, Rises in $\left[\mathrm{Ca}^{2+}\right]_{\mathrm{i}}$ were measured in suspensions of Fura-2-loaded platelets co-stimulated with CVX $(100 \mathrm{ng} / \mathrm{ml})$ and $\operatorname{Thr}(4 \mathrm{nM})$ in the presence of $2 \mathrm{mM} \mathrm{CaCl}$. Preincubation with vehicle or cyclosporin $\mathrm{A}(4 \mu \mathrm{M})$ was as indicated. $C$, Western blot and immunostaining with ${ }_{4} \mathrm{G} 10 \mathrm{mAb}$ of platelet lysates after preincubation with vehicle or cyclosporin $\mathrm{A}(4 \mu \mathrm{M})$, and time-dependent co-stimulation with CVX (100 $\mathrm{ng} / \mathrm{ml})$ plus $\operatorname{Thr}(4 \mathrm{nM})$ or ionomycin $(20 \mu \mathrm{M})$ in the presence of $2 \mathrm{mM} \mathrm{CaCl} 2$. Means \pm S.E. $(n=3) ; * *, p<0.02 \mathrm{com}$ pared to vehicle. Arrow indicates position of Src family proteins.

\section{Discussion}

In this work, we provide new insight into the mechanism of integrin $\alpha$ llb $\beta_{3}$ closure in agonist-induced PS-exposure. In platelets stimulated with convulxin/thrombin, we could establish that initial activation of this integrin is followed by closure at later time points. Twocolor flow cytometry indicated that most of the platelets that expose PS turn off their integrins. Defective adhesion of such platelets was confirmed by single platelet count analysis, showing that the count of single platelets in suspension increased after long term activation with convulxin/thrombin along with PS exposure. 
A
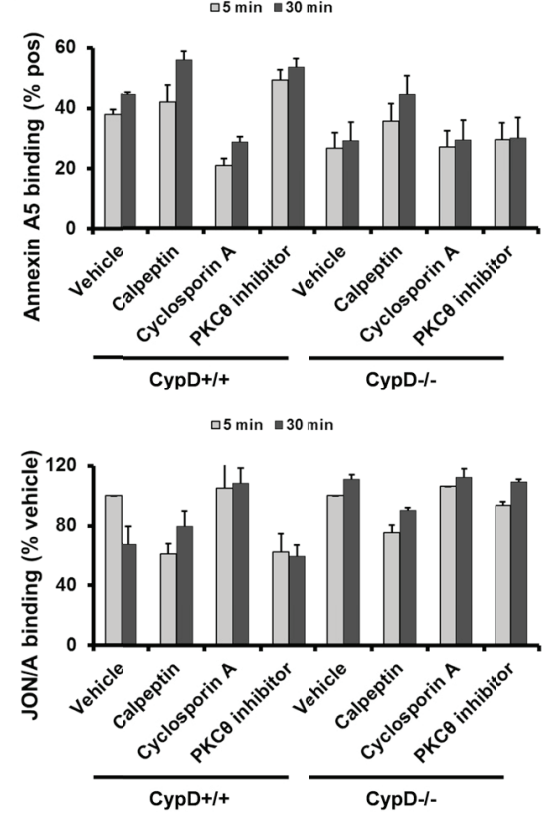

B
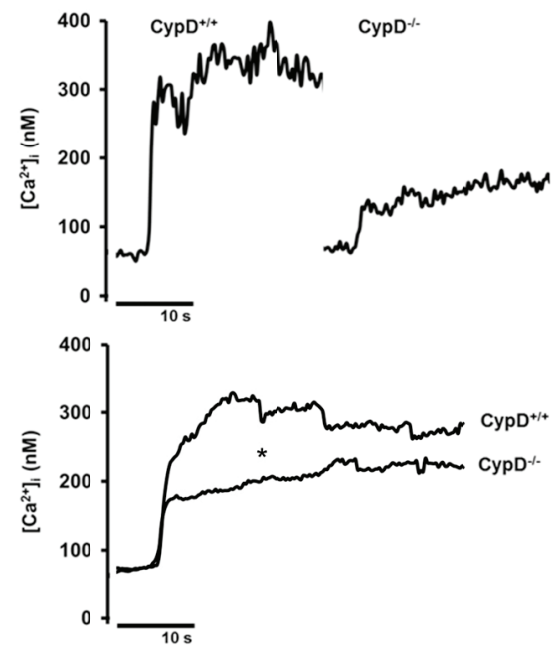

Figure 5. Effects of CypD deficiency on integrin $\alpha \mathrm{llb} \beta_{3}$ closure, $\mathrm{PS}$ exposure and $\mathrm{Ca}^{2+}$ signaling. Washed mouse platelets were stimulated for 5-30 minutes with convulxin $(100 \mathrm{ng} / \mathrm{ml})$ plus thrombin (4 $\mathrm{nM}$ ) in the presence of $2 \mathrm{mM} \mathrm{CaCl} 2$. A, Time-dependent binding of AF647-annexin $\mathrm{A}_{5}$ (upper panel) and $\mathrm{PE}-\mathrm{JON} / \mathrm{A}$ (lower panel) of $\mathrm{CypD}^{+/+}$and $\mathrm{CypD}^{-1 /}$ platelets, measured by flow cytometry. Preincubation was with vehicle $(0.2 \% \mathrm{Me} 2 \mathrm{SO})$, calpeptin $(200 \mu \mathrm{M})$, cyclosporin $\mathrm{A}(4 \mu \mathrm{M})$ or PKC $\theta$ inhibitor $(1 \mu \mathrm{M})$, as indicated. Means \pm S.D. $(n=2)$. B, $\mathrm{Ca}^{2+}$ responses during perfusion over collagen of Fluo-4-loaded platelets from $\mathrm{CypD}^{+/+}$and $\mathrm{CypD}^{-1-}$ mice. Shown are representative $\mathrm{Ca}^{2+}$ traces (upper panel) and average traces from $30-50$ cells (lower panel), *, $\mathrm{p}<0.05$ compared to wildtype.

Mechanistically, our data indicate that integrin closure in PS-exposing platelets coincided with cleavage of the protein kinase Src in a $\mathrm{Ca}^{2+}$-dependent way. Markedly, Src cleavage along with integrin closure was abolished in the presence of the Src-family kinase inhibitor, $\mathrm{PP}_{2}$, suggesting a requirement in integrin closure. However, $\mathrm{Ca}^{2+}$ measurements suggested that this inhibitory effect was due to PP2-inhibited activation of the GPVI/PLCY2 pathway by Src-family kinase members. In contrast, a specific inhibitor of Src did not significantly affect PS exposure and integrin closure. Hence, while Src is cleaved in PS-exposing platelets, this cleavage is not responsible for integrin closure. Since Src-dependent signaling is essential in integrin $\alpha$ llb $\beta_{3}$-induced outside-in signaling (52), these data yet reveal a novel negative feedback pathway in PS-exposing platelets, where abolition of Src functioning via calpain-dependent cleavage blocks integrin-dependent outside-in signaling.

Our findings also modify an earlier proposed scenario that calpain-mediated cleavage of cytoskeletal related proteins is a regular post-aggregation event (24). We show that platelet treatment with the calpain inhibitor calpeptin abrogates Src cleavage, but no more than partly affects integrin closure and PS exposure in response to convulxin/thrombin. In other words, inhibition of calpain under conditions of sustained high intracellular $\mathrm{Ca}^{2+}$ does 
not abolish integrin closure, nor PS exposure. Based on these results another calpainindependent pathway of integrin closure appears to occur in PS-exposing platelets.

In various cell types, mitochondria serve as $\mathrm{Ca}^{2+}$-buffering systems. In platelets, loss of the mitochondrial transmembrane potential and formation of the MPTP appear to contribute to the $\mathrm{Ca}^{2+}$-dependent activation process $(53,54)$. Similarly, platelets from $\mathrm{CypD}^{-1-}$ mice, which cannot form a MPTP, have been reported to be impaired in PS exposure in response to strong agonists (28). In our hands, we find a partial reduction in both integrin closure and PS exposure in mouse platelets lacking CypD, accompanied by a moderate reduction in $\mathrm{Ca}^{2+}$ signaling. In human platelets stimulated with convulxin/thrombin, we find that cyclosporin A treatment causes a substantial reduction in integrin closure and PS exposure, although $\mathrm{Ca}^{2+}$ signaling remains unaffected. These findings do not agree with those of DacharyPrigent et al., who propose that MPTP formation does not play a role in PS exposure (26). Leaving open the possibilities of subtle differences in mechanism between mouse and human platelets and CypD-independent effects of cyclosporin A, our results jointly point to a partial role of $\mathrm{mPTP}$ formation that could occur independently of $\mathrm{Ca}^{2+}$ mobilization. Whether PKC $\theta$ indeed modulates this function of mitochondria - such as suggested by inhibitor experiments - needs to be confirmed.

Taken together, this study reveals partial contributions of calpain and of MPTP formation in agonist-induced integrin closure in PS-exposing platelets.

\section{Acknowledgements}

This work was supported by the Marie Curie program Early Stage Research Training Grant 2005-020706-2.

\section{References}

1 Munnix IC, Kuijpers MJ, Auger J, Thomassen CM, Panizzi P, van Zandvoort MA, Rosing J, Bock PE, Watson SP, \& Heemskerk JW. Segregation of platelet aggregatory and procoagulant microdomains in thrombus formation: Regulation by transient integrin activation. Arterioscler Thromb Vasc Biol 27, 2484-90 (2007).

2 Kulkarni S, Dopheide SM, Yap CL, Ravanat C, Freund M, Mangin P, Heel KA, Street A, Harper IS, Lanza F, \& Jackson SP. A revised model of platelet aggregation. J Clin Invest 105, 783-91 (2000).

3 Heemskerk JW, Bevers EM, \& Lindhout T. Platelet activation and blood coagulation. Thromb Haemost 88, 186-93 (2002).

4 Kuijpers MJ, Munnix IC, Cosemans JM, Vlijmen BV, Reutelingsperger CP, Egbrink MO, \& Heemskerk JW. Key role of platelet procoagulant activity in tissue factor-and collagen-dependent thrombus formation in arterioles and venules in vivo differential sensitivity to thrombin inhibition. Microcirculation 15, 269-82 (2008).

5 Siljander PR, Munnix IC, Smethurst PA, Deckmyn H, Lindhout T, Ouwehand WH, Farndale RW, \& Heemskerk JW. Platelet receptor interplay regulates collagen-induced thrombus formation in flowing human blood. Blood 103, 1333-41 (2004).

6 Kulkarni S \& Jackson SP. Platelet factor XIII and calpain negatively regulate integrin $\alpha$ llb $\beta_{3}$ adhesive function and thrombus growth. J Biol Chem 279, 30697-706 (2004).

7 Cosemans JM, Iserbyt BF, Deckmyn H, \& Heemskerk JW. Multiple ways to switch platelet integrins on and off. J Thromb Haemost 6, 1253-61 (2008).

8 Nieswandt B \& Watson SP. Platelet-collagen interaction: Is GPVI the central receptor? Blood 102, 449-61 (2003).

9 Watson SP, Auger JM, McCarty OJ, \& Pearce AC. GPVI and integrin $\alpha$ llb $\beta 3$ signaling in platelets. J Thromb Haemost 3, 1752-62 (2005). 
Wonerow P \& Watson SP. The transmembrane adapter LAT plays a central role in immune receptor signalling. Oncogene 20,6273-83 (2001).

11 Gilio K, Munnix IC, Mangin P, Cosemans JM, Feijge MA, van der Meijden PE, Olieslagers S, Chrzanowska-Wodnicka MB, Lillian R, Schoenwaelder S, Koyasu S, Sage SO, Jackson SP, \& Heemskerk JW. Non-redundant roles of phosphoinositide 3 -kinase isoforms $\alpha$ and $\beta$ in glycoprotein VI-induced platelet signaling and thrombus formation. J Biol Chem 284, 33750-62 (2009).

12 Schoenwaelder SM, Ono A, Sturgeon S, Chan SM, Mangin P, Maxwell MJ, Turnbull S, Mulchandani M, Anderson K, Kauffenstein G, Rewcastle GW, Kendall J, Gachet C, Salem HH, \& Jackson SP. Identification of a unique co-operative phosphoinositide 3 -kinase signaling mechanism regulating integrin $\alpha \mathrm{llb} \beta 3$ adhesive function in platelets. J Biol Chem 282, 28648-58 (2007).

13 Wagner CL, Mascelli MA, Neblock DS, Weisman HF, Coller BS, \& Jordan RE. Analysis of GPIIb/IIla receptor number by quantification of $7 \mathrm{E}_{3}$ binding to human platelets. Blood 88, 907-14 (1996).

14 Cifuni SM, Wagner DD, \& Bergmeier W. CaIDAG-GEFI and protein kinase $C$ represent alternative pathways leading to activation of integrin $\alpha \mathrm{llb} \beta 3$ in platelets. Blood 112, 1696-703 (2008).

15 Jackson SP, Yap CL, \& Anderson KE. Phosphoinositide 3-kinases and the regulation of platelet function. Biochem Soc Trans 32, 387-92 (2004).

16 Tao L, Zhang Y, Xi X, \& Kieffer N. Recent advances in the understanding of the molecular mechanisms regulating platelet integrin $\alpha$ llb $\beta_{3}$ activation. Protein Cell 1, 627-37 (2010).

17 Partridge AW, Liu S, Kim S, Bowie JU, \& Ginsberg MH. Transmembrane domain helix packing stabilizes integrin $\alpha$ llb $\beta 3$ in the low affinity state. J Biol Chem 280, 7294-300 (2005).

$18 \mathrm{Ma} Y \mathrm{Y}$, Qin J, \& Plow EF. Platelet integrin $\alpha$ llb $\beta_{3}$ : Activation mechanisms. J Thromb Haemost 5 , 1345-52 (2007).

19 Nieswandt B, Varga-Szabo D, \& Elvers M. Integrins in platelet activation. J Thromb Haemost 7 Suppl 1, 206-9 (2009).

20 Shattil SJ \& Newman PJ. Integrins: Dynamic scaffolds for adhesion and signaling in platelets. Blood 104, 1606-15 (2004).

21 Munnix IC, Cosemans JM, Auger JM, \& Heemskerk JW. Platelet response heterogeneity in thrombus formation. Thromb Haemost 102, 1149-56 (2009).

22 Yan B, Calderwood DA, Yaspan B, \& Ginsberg MH. Calpain cleavage promotes talin binding to the $\beta_{3}$ integrin cytoplasmic domain. J Biol Chem 276, 28164-70 (2001).

23 Fox JE. On the role of calpain and Rho proteins in regulating integrin-induced signaling. Thromb Haemost 82, 385-91 (1999).

24 Schoenwaelder SM, Yuan Y, Cooray P, Salem HH, \& Jackson SP. Calpain cleavage of focal adhesion proteins regulates the cytoskeletal attachment of integrin $\alpha$ llb $\beta_{3}$ and the cellular retraction of fibrin clots. J Biol Chem 272, 1694-702 (1997).

25 Smeets EF, Comfurius P, Bevers EM, \& Zwaal RF. Calcium-induced transbilayer scrambling of fluorescent phospholipid analogs in platelets and erythrocytes. Biochim Biophys Acta 1195, 281-6 (1994).

26 Arachiche A, Kerbiriou-Nabias D, Garcin I, Letellier T, \& Dachary-Prigent J. Rapid procoagulant phosphatidylserine exposure relies on high cytosolic calcium rather than on mitochondrial depolarization. Arterioscler Thromb Vasc Biol 29, 1883-9 (2009).

27 Baines CP, Kaiser RA, Purcell NH, Blair NS, Osinska H, Hambleton MA, Brunskill EW, Sayen MR, Gottlieb RA, Dorn GW, Robbins J, \& Molkentin JD. Loss of cyclophilin D reveals a critical role for mitochondrial permeability transition in cell death. Nature 434, 658-62 (2005).

28 Jobe SM, Wilson KM, Leo L, Raimondi A, Molkentin JD, Lentz SR, \& Di Paola J. Critical role for the mitochondrial permeability transition pore and cyclophilin $D$ in platelet activation and thrombosis. Blood 111, 1257-65 (2008).

29 Siljander P, Farndale RW, Feijge MA, Comfurius P, Kos S, Bevers EM, \& Heemskerk JW. Platelet adhesion enhances the glycoprotein $\mathrm{VI}$-dependent procoagulant response: Involvement of $\mathrm{p}_{3} 8$ MAP kinase and calpain. Arterioscler Thromb Vasc Biol 21, 618-27 (2001).

30 Cywin CL, Dahmann G, Prokopowicz AS, 3rd, Young ER, Magolda RL, Cardozo MG, Cogan DA, Disalvo D, Ginn JD, Kashem MA, Wolak JP, Homon CA, Farrell TM, Grbic H, Hu H, Kaplita PV, Liu LH, Spero DM, Jeanfavre DD, O'Shea KM, White DM, Woska JR, Jr., \& Brown ML. Discovery of potent and selective PKC $\theta$ inhibitors. Bioorg Med Chem Lett 17, 225-30 (2007). 
31 Kuijpers MJ, Schulte V, Bergmeier W, Lindhout T, Brakebusch C, Offermanns S, Fassler R, Heemskerk JW, \& Nieswandt B. Complementary roles of glycoprotein VI and $\alpha_{2} \beta_{1}$ integrin in collagen-induced thrombus formation in flowing whole blood ex vivo. FASEB J 17, 685-7 (2003).

32 van der Meijden PE, Schoenwaelder SM, Feijge MA, Cosemans JM, Munnix IC, Wetzker R, Heller $\mathrm{R}$, Jackson $\mathrm{SP}$, \& Heemskerk JW. Dual $\mathrm{P}_{2} \mathrm{Y}_{12}$ receptor signaling in thrombin-stimulated plateletsinvolvement of phosphoinositide 3 -kinase $\beta$ but not $\gamma$ isoform in $\mathrm{Ca}^{2+}$ mobilization and procoagulant activity. FEBS J 275, 371-85 (2008).

33 Lecut C, Schoolmeester A, Kuijpers MJ, Broers JL, van Zandvoort MA, Vanhoorelbeke K, Deckmyn $H$, Jandrot-Perrus $M, \&$ Heemskerk JW. Principal role of glycoprotein VI in $\alpha_{2} \beta_{1}$ and $\alpha$ llb $\beta 3$ activation during collagen-induced thrombus formation. Arterioscler Thromb Vasc Biol 24, 1727-33 (2004).

34 Storey RF, May JA, \& Heptinstall S. Potentiation of platelet aggregation by heparin in human whole blood is attenuated by $\mathrm{P}_{2} \mathrm{Y}_{12}$ and $\mathrm{P}_{2} \mathrm{Y}_{1}$ antagonists but not aspirin. Thromb Res 115, 301-7 (2005).

35 Spronk HM, Soute BA, Schurgers LJ, Cleutjens JP, Thijssen HH, De Mey JG, \& Vermeer C. Matrix Gla protein accumulates at the border of regions of calcification and normal tissue in the media of the arterial vessel wall. Biochem Biophys Res Commun 289, 485-90 (2001).

36 Feijge MA, van Pampus EC, Lacabaratz-Porret C, Hamulyak K, Levy-Toledano S, Enouf J, \& Heemskerk JW. Inter-individual variability in $\mathrm{Ca}^{2+}$ signalling in platelets from healthy volunteers: Effects of aspirin and relationship with expression of endomembrane $\mathrm{Ca}^{2+}$-ATPases. $\mathrm{Br} \mathrm{J}$ Haematol 102, 850-9 (1998).

37 Heemskerk JW, Feijge MA, Henneman L, Rosing J, \& Hemker HC. The $\mathrm{Ca}^{2+}$-mobilizing potency of $\alpha$-thrombin and thrombin-receptor-activating peptide on human platelets - concentration and time effects of thrombin-induced $\mathrm{Ca}^{2+}$ signaling. Eur J Biochem 249, 547-55 (1997).

38 Auger JM, Kuijpers MJ, Senis YA, Watson SP, \& Heemskerk JW. Adhesion of human and mouse platelets to collagen under shear: a unifying model. FASEB J 19, 825-7 (2005).

39 Heemskerk JW, Willems GM, Rook MB, \& Sage SO. Ragged spiking of free calcium in ADPstimulated human platelets: Regulation of puff-like calcium signals in vitro and ex vivo. J Physiol $535,625-35$ (2001).

40 Gilio K, van Kruchten R, Braun A, Berna-Erro A, Feijge MA, Stegner D, van der Meijden PE, Kuijpers MJ, Varga-Szabo D, Heemskerk JW, \& Nieswandt B. Roles of platelet STIM1 and Orai1 in glycoprotein VI- and thrombin-dependent procoagulant activity and thrombus formation. J Biol Chem 285, 23629-38 (2010).

41 Reuter C, Findik D, \& Presek P. Characterization of purified pp6oc-src protein tyrosine kinase from human platelets. Eur J Biochem 190, 343-50 (1990).

42 Bain J, Plater L, Elliott M, Shpiro N, Hastie CJ, McLauchlan H, Klevernic I, Arthur JS, Alessi DR, \& Cohen P. The selectivity of protein kinase inhibitors: A further update. Biochem J 408, 297-315 (2007).

43 Suzuki-Inoue K, Hughes CE, Inoue O, Kaneko M, Cuyun-Lira O, Takafuta T, Watson SP, \& Ozaki $\mathrm{Y}$. Involvement of Src kinases and PLCY2 in clot retraction. Thromb Res 120, 251-8 (2007).

44 Suzuki-Inoue K, Tulasne D, Shen Y, Bori-Sanz T, Inove O, Jung SM, Moroi M, Andrews RK, Berndt MC, \& Watson SP. Association of Fyn and Lyn with the proline-rich domain of glycoprotein $\mathrm{VI}$ regulates intracellular signaling. J Biol Chem 277, 21561-6 (2002).

45 Pasquet JM, Quek L, Pasquet S, Poole A, Matthews JR, Lowell C, \& Watson SP. Evidence of a role for SHP-1 in platelet activation by the collagen receptor glycoprotein VI. J Biol Chem 275, 28526 31 (2000).

46 Pradhan S, Alrehani N, Patel V, Khatlani T, \& Vijayan KV. Cross-talk between serine/threonine protein phosphatase $2 \mathrm{~A}$ and protein tyrosine phosphatase $1 \mathrm{~B}$ regulates Src activation and adhesion of integrin $\alpha$ llb $\beta 3$ to fibrinogen. J Biol Chem 285, 29059-68 (2010).

47 Verhallen PF, Bevers EM, Comfurius P, \& Zwaal RF. Correlation between calpain-mediated cytoskeletal degradation and expression of platelet procoagulant activity. A role for the platelet membrane-skeleton in the regulation of membrane lipid asymmetry? Biochim Biophys Acta 903, 206-17 (1987).

48 Yano Y, Shiba E, Kambayashi J, Sakon M, Kawasaki T, Fujitani K, Kang J, \& Mori T. The effects of calpeptin, a calpain specific inhibitor, on agonist induced microparticle formation from the platelet plasma membrane. Thromb Res 71, 385-96 (1993). 
49 Yan R, Wang Z, Yuan Y, Cheng H, \& Dai K. Role of cAMP-dependent protein kinase in the regulation of platelet procoagulant activity. Arch Biochem Biophys 485, 41-8 (2009).

50 Santo-Domingo J \& Demaurex N. Calcium uptake mechanisms of mitochondria. Biochim Biophys Acta 1797, 907-12 (2010).

51 Kinnally KW, Peixoto PM, Ryu SY, \& Dejean LM. Is mPTP the gatekeeper for necrosis, apoptosis, or both? Biochim Biophys Acta 1813, 616-22 (2010).

52 Shattil SJ. Integrins and Src: Dynamic duo of adhesion signaling. Trends Cell Biol 15, 399-403 (2005).

53 Kile BT. The role of the intrinsic apoptosis pathway in platelet life and death. J Thromb Haemost 7 Suppl 1, 214-7 (2009).

54 Parekh AB. Mitochondrial regulation of intracellular $\mathrm{Ca}^{2+}$ signaling: More than just simple $\mathrm{Ca}^{2+}$ buffers. News Physiol Sci 18, 252-6 (2003). 


\section{Chapter 8}

General discussion 
In this thesis, we have investigated various roads leading to thrombus formation, and by finding new concepts that regulate this process, we contributed to demystifying the roles of earlier suggested platelet signaling pathways. Our investigations involved multiple apects of thrombus formation, from platelet receptors, via the signaling roles of specific protein and lipid kinases, to the molecular components of intracellular $\mathrm{Ca}^{2+}$ regulation. In this respect, we define thrombus formation as the overall result of platelet activation, aggregation, and procoagulant activity during whole blood flow over a thrombogenic surface in vitro or in vivo.

\section{Collagen-dependent thrombus formation as a multiple adhesive receptor process}

Platelets display several surface receptors for binding to collagen, the function of which varies with the prevalent shear stress conditions and the type and fibrillation of the collagen substrate $(1,2)$. By using several combinations of triple-helical peptide substrates, mimicking distinct adhesive domains of collagen, whereby platelets bind to integrin $\alpha_{2} \beta_{1}$, glycoprotein VI (GPVI) or GPIb-V-IX/von Willebrand factor (VWF), we established that platelet binding to multiple collagen receptors is required for full thrombus formation in particular at high shear, arterial shear rate. In chapter 2 , we show that two collagen-mimetic peptide sequences, GFOGER and (GPO) $)_{n}$ - binding to $\alpha_{2} \beta_{1}$ and GPVI, respectively - , in combination with immobilized vWF causes full thrombus formation. The combination of these three substrate sequences induces similar platelet responses as native collagen type I fibers. Furthermore, with the triple-helical peptide(GPO) $)_{n}$-GFOGER-(GPO) $)_{n}$, we find a similar pattern of heterogeneity of aggregated platelets versus single phosphatidylserine (PS) exposing platelets as was detected earlier upon whole blood perfusion over native collagen fibers (3). As indicated in Figure 1, full-blown formation of stable thrombi under flow requires the combination of VWF and collagen-mimetic peptides with binding sites for both $\alpha_{2} \beta_{1}$ and GPVI. Hence, those triple-helical GXX'GER(GPO) $n$ sequences that bind with highest affinity to $\alpha_{2} \beta_{1}$ are also the most active ones in supporting thrombus formation and platelet procoagulant activity at given shear conditions (Figure 1 ). This synergy between a signaling receptor for collagen, GPVI, and (high-shear) adhesion receptors, i.e $\alpha_{2} \beta_{1}$ and GPIb-VIX/VWF, has also be noted by others (4). By consequence, our findings support and extend the earlier defined principle of interplay between these three receptors in stable platelet adhesion and activation at arterial shear rate (5-7). In this respect, we like to note that the earlier data were obtained by immunological blocking of $\alpha_{2} \beta_{1}, G P V I$, or GPIb, or by using mice lacking one of the receptors, i.e. conditions where side effects due to non-specific antibody inhibition or to down-regulation of other receptor-mediated responses cannot be excluded. 


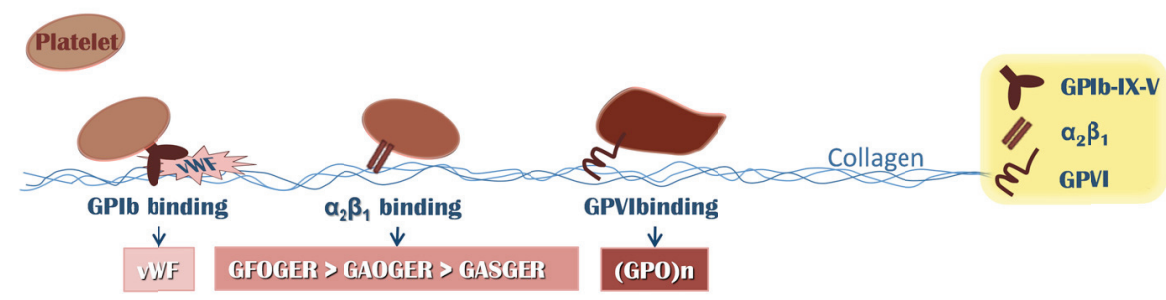

\begin{tabular}{|c|c|c|c|}
\hline W'y & & & No platelet adhesion \\
\hline & GEOGES\} & (GPP)n & Small thrombi, procoagulant activity \\
\hline & & (GPO)n & No platelet adhesion \\
\hline עУУF & GIFOGES & (GPP)n & Instable thrombi, procoagulant activity \\
\hline บУУЕ & GFDGES & $(G P D) n$ & Stable thrombi, procoagulant activity \\
\hline
\end{tabular}

\begin{tabular}{|c|c|c|c|}
\hline & GADEES - CASGES & (GPP)n & No platelet adhesion \\
\hline W'Y & GALCEES - GASGES & $(\mathrm{GPO}) n$ & Stable thrombi, procoagulant activity \\
\hline
\end{tabular}

Figure 1. Immobilized collagen-mimetic peptides in thrombus formation at high-shear blood flow. Summary of the results from chapter 2 on thrombus formation induced by various immobilized triplehelical collagen-mimetic peptides, binding to platelet integrin $\alpha_{2} \beta_{1}\left(G X X^{\prime} G E R\right)$ or GPVI [(GPO)n with $(G P P)_{n}$ as negative control]. Upper part compares the different affinities of GXX'GER hexapeptides for activated integrin $\alpha_{2} \beta_{1}$ according to ref. [7]. The indicated peptides were immobilized alone or together with vWF. Stable thrombus formation with procoagulant, PS-exposing platelets - resembling the thrombi formed on native type I collagen - was obtained with the combinations of GFOGER(GPO) $n$ GAO/SGER(GPO) $n$ plus vWF. In the absence of vWF, only GFOGER(GPO) $)_{n}$ with high affinity for $\alpha_{2} \beta_{1}$ was limitedly active in supporting thrombus formation. Immobilization of (GPO) n peptide or vWF alone did not result in platelet adhesion.

An unpredicted finding was that the $\alpha_{2} \beta_{1}$-binding triple-helical peptide, $(G P P)_{n}$ GFOGER-(GPP) $)_{n}$ lacking the GPVI-activating (GPO) $)_{n}$ sequence, did weakly induce instable platelet aggregation and PS exposure in both high- and low-shear flow settings (Figure 1). Detailed analysis by the Farndale group indicated that such triple-helical (GPP) $n$ peptides have an extremely low affinity for GPVI (8), thus proposing that the GFOGER sequence is responsible for the platelet-activating effect. We also found that blocking of GPVI suppressed the platelet responses, suggesting that GFOGER(GPP) ${ }_{n}$ surfaces have a low GPVIdependent activity.

These results are reminiscent to those from others, showing that static adhesion of platelets to triple helical GFOGER hexapeptide (9) provokes tyrosine phosphorylation of various signaling proteins of the GPVI-Fc receptor $\gamma$-chain (FcRY) cascade, including Src, Syk, SLP76 and phospholipase $C_{Y}(P L C Y)$ (10). These authors suggested that integrin $\alpha_{2} \beta_{1}$ engagement via outside-in signaling leads to a similar activation pathway as does GPVI ligation. Another, recent paper showed that $\alpha_{2} \beta_{1}$-mediated adhesion of platelets to 
GFOGER peptides causes activation of the pathway of phosphatidylinositol 3-kinase $\beta$ $\left(\mathrm{Pl}_{3} \mathrm{KK} \beta\right.$ ) and $A$ kt (11). Because $\alpha_{2} \beta_{1}$ by itself is unable to stimulate $\mathrm{Pl}_{3} \mathrm{~K} \beta$ (11) and, as shown in chapter 3 of this thesis, GPVI/PLC $\gamma$ induces prominent $\mathrm{Pl}_{3} \mathrm{KK} \beta$ activation and downstream signaling, these findings are supportive for a role of $\alpha_{2} \beta_{1}$ engagement with GFOGER in the activation of GPVI, possibly via a trans-receptor signaling mechanism. Support for this idea comes from the finding that both receptors appear to interact during collagen activation of platelets (6). However, future research is needed to establish how precisely such receptor cross-talk and trans-receptor signaling can take place in the platelet membrane.

\section{Roles of $\mathrm{Pl}_{3} \mathrm{~K}$ and $\mathrm{PKC}$ isoforms in thrombus formation and procoagulant activity}

Ligand binding to collagen and other platelet receptors leads to complex networks of signaling processes that eventually result in functional responses, such as integrin $\alpha$ llb $\beta_{3}$ activation, secretion, platelet aggregation, and procoagulant activity. Because they are placed at crucial nodes in these networks, two families of signaling proteins have shown to be of great importance. These are the families of phosphoinositide 3 -kinase $\left(\mathrm{Pl}_{3} \mathrm{~K}\right)$ and protein kinase $C(P K C)$, both of which are studied in detail in this thesis. Like other crucial signaling proteins, more than one isoform of either family is present in platelets. In order to study the roles of individual $\mathrm{PI}_{3} \mathrm{~K}$ and $\mathrm{PKC}$ isoforms, and possible redundancy therein, we considered it important to follow both pharmacological and genetic approaches. The reason for this is that pharmacological treatment of human cells with so-called isoform-specific inhibitors has the risk of limited sensitivity and specificity of the inhibitors, whereas in studies with kinase deficient mice is it difficult to rule out that other relevant (family member) proteins are up- or down-regulated in a compensatory way.

Table 1 gives an overview of key roles of the different class 1 PI3K isoforms in platelet responses, in particular those we have been able to establish in this thesis. Considering GPVI-dependent thrombus formation under flow conditions, both pharmacological inhibition of $\mathrm{Pl}_{3} \mathrm{~K} \alpha / \beta$ and genetic deficiency of a key regulatory subunit for both isoforms results in lesser platelet adhesion to collagen fibers, reduced platelet aggregation, suppression of platelet $\mathrm{Ca}^{2+}$ fluxes, and lower PS exposure. On the other hand, inhibition of $\mathrm{Pl}_{3} \mathrm{~K} Y$ did not affect GPVI-induced $\mathrm{Ca}^{2+}$ responses, while inhibition of $\mathrm{Pl}_{3} \mathrm{~K} \delta$ caused only a slight reduction. This fully supports the data from others that genetic deficiency in murine p110y (12) or p110 $\delta$ (13) has no more than a modest role in GPVI-induced platelet activation. Retrospectively, these data indicate that the $\mathrm{PI}_{3} \mathrm{~K}$ inhibitors used were sufficiently specific for the concerning $\mathrm{Pl}_{3} \mathrm{~K}$ isoforms.

Mechanistically, the experiments indicate that both $\mathrm{Pl}_{3} \mathrm{~K} \alpha$ and $\beta$ isoforms are needed for full PLC $\gamma$ activity and inositol 1,4,5-trisphosphate (IP3) formation. This pointed to a nonredundant role of both isoforms in activation of this key effector enzyme of GPVI. This is also supported by the contribution of these $\mathrm{Pl}_{3} \mathrm{~K}$ isoforms to $\mathrm{Ca}^{2+}$ mobilization from intracellular stores, which is a direct consequence of PLC activation, rather than to subsequent $\mathrm{Ca}^{2+}$ entry from outside the platelets. Hence, we reasoned that the earlier suggested stimulation of $\mathrm{Ca}^{2+}$ entry by $\mathrm{Pl}_{3} \mathrm{~K}$ or its product, phosphatidylinositol 3,4,5-trisphosphate $(14,15)$, is secondary to the effect of $\mathrm{Pl}_{3} \mathrm{~K}$ on $\mathrm{Ca}^{2+}$ store depletion. 
Table 1. Established functions of $\mathrm{Pl}_{3} \mathrm{~K}$ isoforms in platelet activation processes and thrombus formation. Overview of chapter 3 regarding modulating roles of various $\mathrm{Pl}_{3} \mathrm{~K}$ isoforms on GPVI-induced platelet responses (directly or indirectly via $\mathrm{P}_{2} \mathrm{Y}_{12}$ ). Functions of isoforms were studied using mice deficient in p85 (regulatory subunit of class IA isoforms). Pharmacological inhibitors used were PIK-75 (blocking $\mathrm{Pl}_{3} \mathrm{~K} \alpha$ ), TGX-221 (blocking $\mathrm{Pl}_{3} \mathrm{~K} \beta$ ), IC-87114 (blocking $\mathrm{Pl}_{3} \mathrm{~K} \delta$ ) and $\mathrm{AS}-252424$ (blocking $\mathrm{Pl}_{3} \mathrm{~K} \gamma$ ). Findings not in this thesis but from the literature are indicated. See also text.

\begin{tabular}{|c|c|c|c|c|}
\hline \multirow[b]{2}{*}{ Platelot responses } & \multicolumn{3}{|c|}{ Class 1A } & \multirow{2}{*}{$\begin{array}{l}\text { Class1 B } \\
\text { PJBKY }\end{array}$} \\
\hline & PJ3Ka & P13K $\beta$ & PI3K5 & \\
\hline Akt activation & + & + & ND & ND \\
\hline $\mathrm{IP}_{3}$ production & + & + & 0 & 0 \\
\hline $\mathrm{Ca}^{2+}$ rise & + & + & + & 0 \\
\hline$\alpha_{1 \mid b} \beta_{3}$ activation & + & + & 0 & +1 \\
\hline Secretion ( $\alpha / \delta$-granules) & + & + & ND & $\mathrm{O}^{2}$ \\
\hline Thrombus formation & + & + & 0 & 0 \\
\hline Procoagulant activity & + & + & 0 & 0 \\
\hline
\end{tabular}

${ }^{1}$ Cosemans J, Blood 2006;108:3045-52. ${ }^{2}$ Lian L, Blood 2005;106:110-7. Abbreviations: +, stimulation; 0, no effect; ND, not determined.

The small GTPase Rapıb is known to be activated in a $\mathrm{Pl}_{3} \mathrm{~K}$-dependent manner, particularly after occupation of Gi-coupled receptors, such as the platelet $\mathrm{P}_{2} \mathrm{Y}_{12}$ receptor for $\operatorname{ADP}(16,17)$. We were interested to determine whether GPVI is directly implicated in platelet Rap1b activation, in experiments where the contribution of $\mathrm{P}_{2} \mathrm{Y}_{12}$ receptors was pharmacologically blocked. Such a GPVI-dependent effect may be attributed to the $\mathrm{Pl}_{3} \mathrm{~K} \alpha$ and $\beta$ isoforms. Interestingly, also in Rap1 $\mathrm{b}^{-/-}$mouse platelets, $\mathrm{Pl}_{3} \mathrm{~K}$ inhibition still had a prominent suppressive effect on GPVI-induced integrin $\alpha$ llb $\beta_{3}$ activation and platelet aggregation. This pointed to a role of both $\mathrm{Pl}_{3} \mathrm{~K}$ isoforms downstream of GPVI, in part through a Rap1bindependent pathway. This pathway may again involve $\mathrm{Pl}_{3} \mathrm{~K} \alpha / \beta$-dependent stimulation of

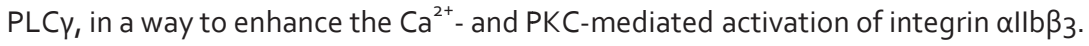

Concerning the PKC family of signaling proteins, isoform-specific inhibitors are less well characterized than those of $\mathrm{Pl}_{3} \mathrm{~K}$. Again, we used pharmacological and genetic approaches to study the roles of the four major platelet-expressed PKC isoforms $(\alpha, \beta, \delta$, and $\theta$ ) in regulating GPVI-induced platelet activation, thrombus formation and procoagulant activity (Table 2). A key finding in collaboration with the Poole laboratory in Bristol (United Kingdom), was a major suppression of thrombus formation with PKC $\alpha$-deficient platelets, whereas platelet adhesion and spreading on collagen was unchanged. The data also reveal 
an important role for PKC $\alpha$ in platelet granule biogenesis and exocytosis (Chapter 4 ). The impaired secretion could explain the suppressed thrombus formation of $\mathrm{PKCa}^{-1-}$ platelets, since secretion could be rescued by the addition of exogenous ADP. Collectively, it was concluded that, during thrombus formation, secretion of granules, integrin $\alpha$ llb $\beta_{3}$ activation, and platelet-platelet interactions are all positively regulated by $\mathrm{PKC} \alpha$. Recent findings of the Bristol lab added to this by demonstrating that protein kinase $D_{2}$ is a key component in the regulation of dense granule secretion, downstream of GPVI-induced PKC $\alpha$ activation (18).

Table 2. Established functions of PKC isoforms in platelet activation processes and thrombus formation. Overview of chapters 4-5 regarding modulating roles of various PKC isoforms on GPVI-induced platelet responses (directly or indirectly via $\mathrm{P}_{2} \mathrm{Y}_{12}$ ). Functions of isoforms were studied using mice deficient in $\mathrm{PKC} \alpha, \beta, \delta$, or $\theta$. Pharmacological inhibitors used were Gö6g76 (blocking $P K C \alpha / \beta$ ), $\beta$ inhibitor (blocking PKC $\beta>\alpha$ ), rottlerin (blocking PKC $\delta$ ) and $\theta$-inhibitor (blocking PKC $\theta$ ). Findings not in this thesis but from the literature are indicated. See also text.

\begin{tabular}{|l|c|c|c|c|}
\hline & \multicolumn{3}{c}{ CPKC } & \multicolumn{2}{c|}{ DPKC } \\
\hline Platglot regponses & PKCa & PKC $\beta$ & PKC & PKCO \\
\hline Filopod formation & 0 & +1 & - & - \\
\hline $\mathrm{Ca}^{2+}$ rise & + & + & 0 & - \\
\hline$\alpha_{11 \mathrm{~b}} \beta_{3}$ activation & + & + & 0 & -4 \\
\hline Secretion ( $\alpha$-granules) & + & + & 0 & $0^{4}$ \\
\hline Secretion ( $\delta$-granules) & + & ND & - & - \\
\hline Aggregation & + & + & - & - \\
\hline Thrombus formation & + & + & - & - \\
\hline Procoagulant activity & + & + & 0 & - \\
\hline
\end{tabular}

${ }^{1}$ Buensuceso CS, J. Biol Chem 2005;278:44369-76. 2Pula G, Blood 2006;108:4035-4. 35oriani A, J. Thromb. Haemost 2006;4:648-55. ${ }^{4} \mathrm{Hall} \mathrm{KJ}$, Plos One 2008;3,e3277. Abbreviations: +, stimulation; -, inhibition; 0, no effect; ND, not determined.

Mouse platelets lacking PKC $\beta$ also formed significantly smaller thrombi on collagen under flow conditions in comparison to wildtype platelets. Platelets from both $P K C \alpha$ - and $\beta$ deficient mice furthermore showed a reduced collagen-induced $\mathrm{Ca}^{2+}$ mobilization and diminished procoagulant activity. These findings were supported by pharmacological experiments with human platelets pretreated with the compounds Gö6976 and PKC $\beta$ inhibitor. Both compounds, with confirmed inhibitory effects on $P K C \alpha / \beta$, suppressed collagen- 
induced platelet aggregation as well as GPVI-induced integrin activation, P-selectin expression, and $\mathrm{Ca}^{2+}$ signal generation.

On the other hand, various pieces of evidence point to a negative role of PKC $\theta$ in thrombus formation. Platelets from mice deficient in PKC $\theta$ showed increased collagendependent granule secretion, $\mathrm{Ca}^{2+}$ signaling and procoagulant activity, cumulating in enhanced thrombus formation (Table 2). Consistent with this, a pharmacological PKC $\theta$ inhibitor increased GPVI-induced human platelet aggregation as well as $\alpha$ llb $\beta_{3}$ activation, $\mathrm{Ca}^{2+}$ mobilization and $\alpha$-granule secretion (Chapter 5 ). A similar, although less prominent increase in thrombus formation was seen with blood from $\mathrm{PKC} \delta^{-1-}$ mice. One report suggests that GPVI-induced granule secretion is increased rather than decreased in $\mathrm{PKC} \delta^{-1}$ mice (19), but we have not been able to confirm this. Data from the Bristol group also point to a potentiated aggregation response with collagen through enhanced filopod formation in $\mathrm{PKC} \delta^{-1-}$ platelets (20). Also for $\mathrm{PKC} \delta$, we obtained confirming pharmacological evidence, since the compound rottlerin appeared to enhance human platelet aggregation. Although rottlerin may not act as an inhibitor for PKC $\delta$ only, as reported in some papers, its gain-of-function effect on platelets is striking and compatible with the increased thrombus formation in $\mathrm{PKC} \delta^{-1-}$ mice. All together, we come to a negative regulatory role of both PKC $\theta$ and $\delta$ in collagen-dependent thrombus formation, although both enzymes differ in their precise action mechanism. Given the most striking phenotype of $\mathrm{PKC}{ }^{-/}$platelets, it appears that this isoform is responsible for the established negative effect of general PKC inhibition on GPVI-induced platelet $\mathrm{Ca}^{2+}$ signaling and procoagulant activity (21), whereas $\mathrm{PKC} \alpha$ is the main positive regulator of secretion and integrin activation.

While in our hands both the pharmacological approach (human platelets) and genetic approach (mouse platelets) point to a negative role for $\mathrm{PKC} \theta$, different findings have been reported by the Kunapuli group (22). These authors found reduced thrombus formation in $\mathrm{PKC}^{-/}$mice in an in vivo model of $\mathrm{FeCl}_{3}$-induced carotid artery injury. This discrepancy may be due to a contribution of thrombin, for it was shown that PKC $\theta$ positively contributes to thrombin-induced signaling in platelets $(22,23)$. Indeed, next to collagen, also thrombin appears to control the thrombotic process in $\mathrm{FeCl}_{3}$-induced carotid injury (24). A next challenge is to establish the contribution of PKC $\theta$ in (patho)physiological thrombus formation in the human system.

In conclusion, our data indicate that the two conventional PKC isoforms $\alpha$ and $\beta$ are non-redundant and essential for collagen-induced granule secretion, a sustained $\mathrm{Ca}^{2+}$ response, and subsequent PS exposure, while especially the novel isoform PKC $\theta$ negatively regulates these processes. By implication, when conventional PKC signaling is restricted, there is no granule secretion for $\mathrm{PKC} \theta$ to regulate. In future work, it is important to find the molecular protein substrates by which these different PKC isoforms regulate platelet functions. 


\section{Determinants of $\mathrm{Ca}^{2+}$ signaling, PS exposure, and integrin closure in thrombus for- mation}

The results of chapter 6 support the literature identifying essential roles for the $\mathrm{Ca}^{2+}$ sensor protein Stimı (in the reticular membrane), and the $\mathrm{Ca}^{2+}$-selective ion channel Orai1 (in the platelet plasma membrane), in the process of collagen-dependent thrombus formation and PS exposure (Figure 2). These results, thus, provide a mechanistic explanation why arterial thrombus formation in vivo is impaired in mice with dysfunctional Stim1 (25) or Orai1 $(26,27)$. By comparing platelets deficient in either Stim1 or Orai1, and finding remarkably similar effects of either knockout, our findings also support the concept of direct interactions of both proteins. The store-operated $\mathrm{Ca}^{2+}$ entry (SOCE) pathway, mediated by Stim1 and Orai1, controls platelet function. In other words, the biochemical interaction between Stim 1 and Orai1, such as established in other cell types (28), translates to similar functional roles in platelet physiology.

The data of chapter 6 also show that both Stim1 and Orai1 tend to become redundant under conditions where high levels of thrombin are generated and a solid thrombus is formed, rich in PS-exposing platelets and fibrin clots. These findings point to the presence of other signaling mechanisms in thrombus formation, operating alongside of SOCE, under conditions of high thrombin generation and, likely, high thrombin-induced platelet activation.

There is indeed evidence for the presence of another $\mathrm{Ca}^{2+}$ entry pathway in platelets that acts in a thrombin receptor-operated fashion. For instance, the imidazole antagonist, SKF96365, which is a well-studied inhibitor of $\mathrm{Ca}^{2+}$ entry in platelets (29), still suppresses the $\mathrm{Ca}^{2+}$ and procoagulant responses of mouse platelets deficient in Stim1 or Orai1 (Chapter 6). Literature shows that thrombin-induced $\mathrm{Ca}^{2+}$ signaling and PS exposure in human platelets does not only rely on SOCE, but also on a mechanism of receptor-operated $\mathrm{Ca}^{2+}$ entry (ROCE) (30). The alternative $\mathrm{Ca}^{2+}$ entry route most likely does not involve the Trpc1 channel, as was proposed once by Rosado (31). Platelets from Trpc1 ${ }^{-1-}$ mice appear to have a completely intact $\mathrm{Ca}^{2+}$ signaling machinery and a normal activation repertoire (32). More likely targets of SKF96365 are the non-SOCE channels, Trpc6 and Orai3, both of which are expressed in platelets and act in a receptor-operated way $(33,34)$. In mice, a role of Trpc6 has recently been established in thrombin-induced ROCE, although the same study suggested that this platelet protein does not have a major role in hemostasis and thrombosis (35). Another, earlier proposal is that thrombin modulates the $\mathrm{Ca}^{2+}$ rises in platelets by transient activation of the $\mathrm{Na}^{+} / \mathrm{H}^{+}$exchanger (36). 


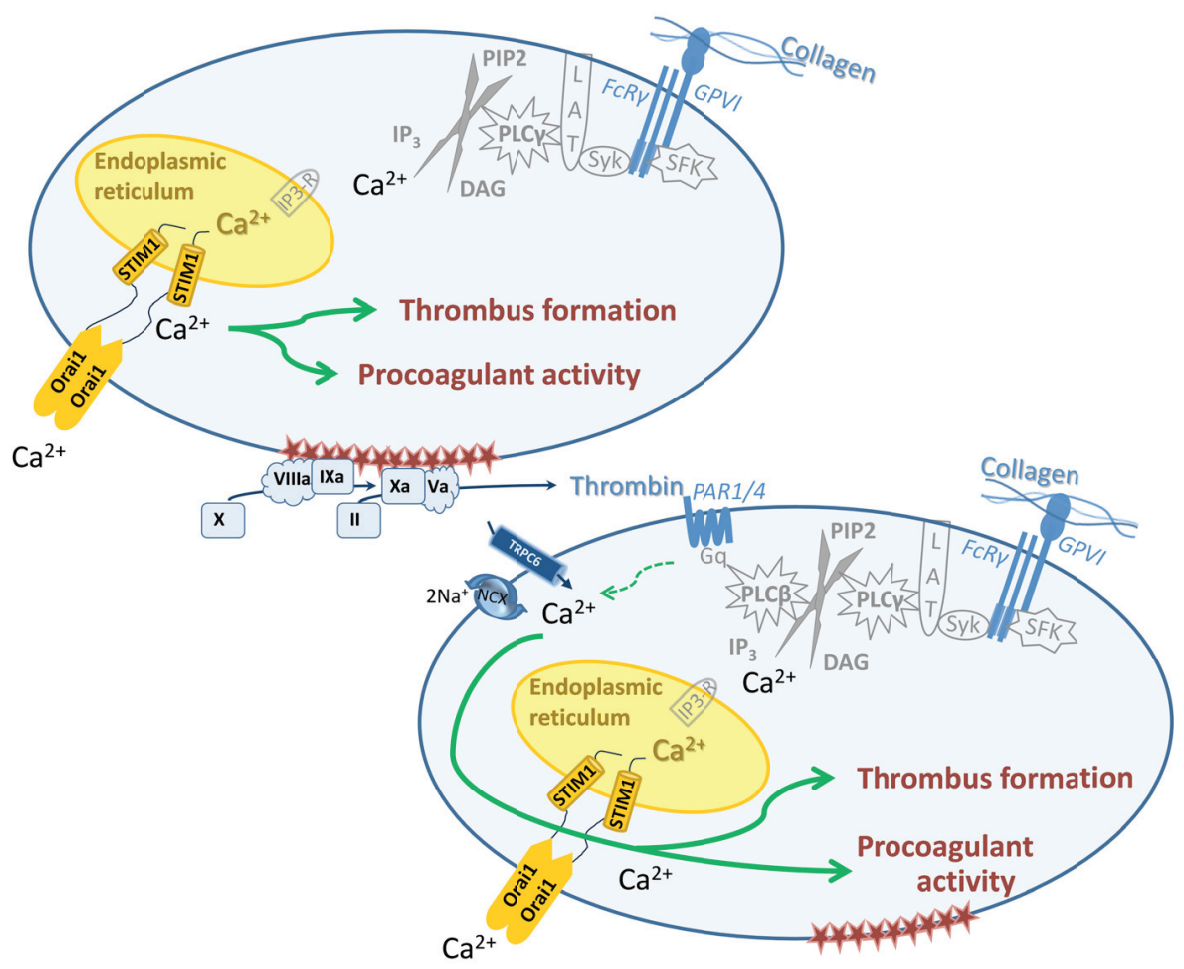

Figure 2. Calcium-modulating proteins in GPVI- and thrombin-dependent thrombus formation and procoagulant activity. Conclusive scheme from chapter 6 on the roles of Orai1 and STIM1 in platelet cytosolic $\mathrm{Ca}^{2+}$ rises, thrombus formation and procoagulant activity. Upper platelet: GPVI-induced platelet stimulation occurs via the LAT signalosome, involving the protein kinase Syk and Src-family kinases (SFK), resulting in activation of phospholipase $\mathrm{C}_{2}(\mathrm{PLC} \gamma)$ and $\mathrm{PP}_{3}$-mediated $\mathrm{Ca}^{2+}$ mobilization. Calcium entry via the SOCE channel Orai1, in interaction with $\mathrm{Ca}^{2+}$-sensor $\mathrm{STIM}_{1}$, is essential for thrombus formation under high-shear flow and for induction of platelet PS exposure. PS exposing platelets provide a procoagulant surface for activation of factor X (complex of factors VIIla, IXa and X), and activation of prothrombin (complex of factors II, Va and Xa). Lower platelet: In the presence of high thrombin concentrations, Orai1 and STIM 1 become redundant in GPVI-induced thrombus formation and procoagulant activity. Thrombin-receptor $\mathrm{PAR} 1 / 4$ stimulation via $\mathrm{Gq}$ and $\mathrm{PLC} \beta$ causes extra $\mathrm{Ca}^{2+}$ signal generation, which may include $\mathrm{Ca}^{2+}$ entry via the $\mathrm{Na}^{+} / \mathrm{Ca}^{2+}$ exchanger (NCX) or the Trpc6 nonSOCE channel. Other abbreviations: DAG, diacylglycerol; PIP 2, phosphatidylinositol 4,5-bisphosphate.

Since SOCE-devoid platelets are still capable to expose PS upon combined collagenand thrombin-receptor stimulation, we rule out a direct role of SOCE in the mechanism of transmembrane scrambling of phospholipids, i.e. the process responsible for PS exposure. This is in agreement with studies in which platelets from Scott syndrome patients, who lack the transmembrane protein TMEM16F (37), have a clear defect in PS exposure, but show unchanged $\mathrm{Ca}^{2+}$ signals in response to collagen and thrombin (38).

Inhibition of $\mathrm{Pl}_{3} \mathrm{~K} \alpha$ or $\beta$ in collagen-adhered platelets led to a reduced $\mathrm{Ca}^{2+}$ mobilization and a lower SOCE and PS exposure during whole-blood perfusion experiments (Chapter 3). Both pharmacological evidence (39) and data with mouse platelets (40) indicated that PKC $\alpha$ can indirectly enhance SOCE by regulating the $\mathrm{Na}^{+} / \mathrm{Ca}^{2+}$ exchanger. In this light, the 
isoform PKCO seems to be an exceptional kinase, because it was found to modify $\mathrm{Ca}^{2+}$ signaling and procoagulant activity in a non-SOCE fashion (41). In our experiments, we found that PKC $\theta$ modulates not only GPVI-induced $\mathrm{Ca}^{2+}$ signaling and subsequent PS exposure but also the closure of integrin $\alpha \mathrm{llb} \beta_{3}$ (Chapter 7).

Figure 3 schematizes the central regulatory function of cytosolic $\mathrm{Ca}^{2+}$, particularly with regard to the platelet responses studied in this thesis. Persistent $\mathrm{Ca}^{2+}$ elevation is a main controlling factor of the closure of activated integrins in PS-exposing platelets, stimulated with collagen and thrombin receptor-activating agonists. The integrin closure coincides with cleavage of the tyrosine kinase Src, a protein that is attached to the integrin $\beta_{3}$ subunit. However, the cleavage of Src appeared to be an epiphenomenon, because Src kinase blockers were without any effect on $\alpha$ llb $\beta_{3}$ closure. Earlier data show that Src is cleaved as a physiologic post-aggregation event, due to action of the $\mathrm{Ca}^{2+}$-dependent protease calpain (42). Furthermore, calpain also mediates cleavage of the integrin $\beta_{3}$ subunit $(43,44)$. Surprisingly, we found that calpain inhibitors were unable to completely suppress integrin closure. Accordingly, calpain-mediated remodeling of the actin cytoskeleton in platelets is not the main or only mechanism of integrin closure.

In many cell types, the mitochondria serve as a $\mathrm{Ca}^{2+}$ buffering system for avoiding cytosolic $\mathrm{Ca}^{2+}$ overload (45). Thus, also in platelets, mitochondria may help to dampen the high rises in cytosolic $\mathrm{Ca}^{2+}$ that are evoked by strong agonists. Formation of the mitochondrial permeability transition pore (MPTP) abolishes the $\mathrm{Ca}^{2+}$-dampening effect, because this allows leakage of the buffered $\mathrm{Ca}^{2+}$ out of the mitochondria (Figure 3). Compatible with this hypothesis, we found that inhibition of MPTP formation (cyclosporin A) or inability to MPTP formation (mice lacking the mPTP component cyclophilin D) resulted in impaired PS exposure and integrin closure. Findings in support of this, although made in different settings, have been published by at least two other groups $(46,47)$.

Although the role of mitochondria in the signaling machinery is only poorly understood, several pieces of evidence in the literature indicate that these organelles contribute to $\mathrm{Ca}^{2+}$ flux regulation and subsequent responses. In neuronal cells, mitochondrial release of $\mathrm{Ca}^{2+}$ due to mPTP formation was found to contribute to cytosolic $\mathrm{Ca}^{2+}$ overload and cellular stress responses (48). On the long-term, mitochondrial-derived $\mathrm{Ca}^{2+}$ signals appear to regulate PKC activity (49). Given that mitochondria are a major source of reactive oxygen species (50), they may also regulate $\mathrm{Ca}^{2+}$ fluxes and PKC activation via the release of hydrogen peroxide (51). Taken together, we envision a scheme that, in platelets stimulated with strong agonists, formation of the MPTP leads to a secondary rise in cytosolic $\mathrm{Ca}^{2+}$ levels, which potentiates PS exposure at the one hand, and activation of the $\mathrm{Ca}^{2+}$-dependent protease calpain and cleavage of Src and $\beta_{3}$ at the other hand. Both processes together, perhaps with an assistant role of PKC $\theta$, may lead to integrin closure (Figure 3 ). Whether PS exposure per se also contributes to integrin closure has not been investigated. 


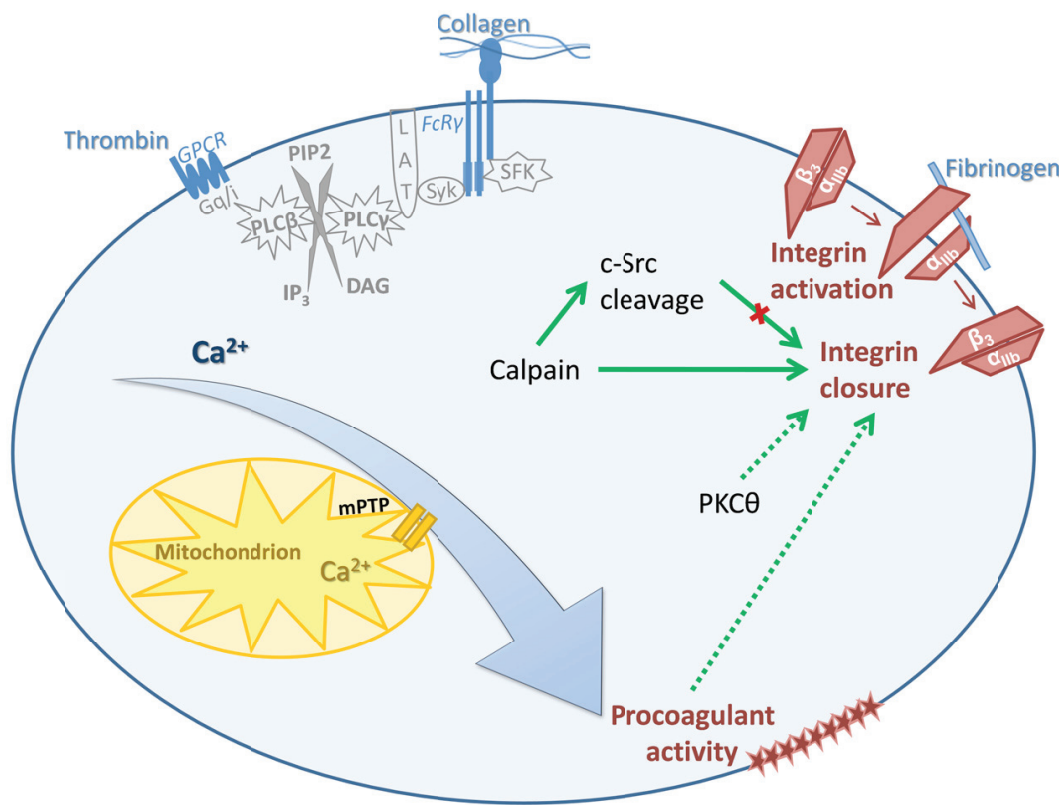

Figure 3. Proposed mechanism of integrin $\alpha \mathrm{llb} \beta_{3}$ closure in procoagulant platelets. As concluded in chapter 7, various routes can lead to integrin closure in PS-exposing platelets stimulated by collagen and thrombin receptors. Agonist-induced $\mathrm{Ca}^{2+}$ rises activate the protease calpain, which is responsible for c-Src kinase cleavage and in part for integrin closure. Mitochondrial-derived $\mathrm{Ca}^{2+}$ release via the mitochondrial permeability transition pore (mPTP) supports the general rise in $\mathrm{Ca}^{2+}$ leading to PS exposure, which process by itself may also contribute to integrin closure. An additional role of PKC $\theta$ is proposed as well. Abbreviations: see Figure 2.

\section{Clinical implications}

Basic medical science aims to disclose the molecular mechanisms of disease and treatment of disease in order to improve human well-being. Several of the platelet signaling proteins studied in this thesis may serve as molecular targets for the treatment of thrombosis or impaired hemostasis.

Although mostly investigated in genetically modified mice, the Orai1 channel has been postulated as a new pharmacological target to reduce platelet activation at sites of arterial thrombosis $(25,26)$. Recent work from our group has shown that SOCE blockers targeted at Orai1 can also suppress thrombus formation of human platelets in vitro, but improvement of the sensitivity and effectiveness of the current compounds appears to be needed before investigations can start towards the prevention of human thrombotic events (52).

Isoforms of both $\mathrm{PKC}$ and $\mathrm{Pl}_{3} \mathrm{~K}$ are among the most frequently upregulated kinases in human tumor tissues (53). Recent insight into the precise functions of $\mathrm{Pl}_{3} \mathrm{~K}$ (54) and $\mathrm{PKC}$ (55) isoforms has increased the interest for these kinases as therapeutic targets not only for anticancer strategies, but also for antithrombotic treatment. In fact, all current antiplatelet drugs (aspirin, $\mathrm{P}_{2} \mathrm{Y}_{12}$ inhibitors, integrin $\alpha$ llb $\beta_{3}$ antagonists) have as side effects impairment of normal hemostasis and a risk of bleeding complications (56). An ideal antithrom- 
botic therapy would selectively suppress the role of platelets in pathological thrombus formation but not affect hemostasis. Experimental thrombosis studies with mice and other laboratory animals have indicated that this might be the case for inhibitors of $\mathrm{Pl}_{3} \mathrm{~K} \beta$ and $\mathrm{Pl}_{3} \mathrm{~K} Y$ (57,58). The compound, AZD6482, a reversible isoform-selective inhibitor of $\mathrm{Pl}_{3} \mathrm{KK} \beta$ has been tested in phase 1 trials and was found to be acceptably safe with a satisfying tolerability profile (59). However, the clinical research with this compound has now been discontinued for commercial reasons. Recent literature demonstrates opposing roles of $\mathrm{Pl}_{3} \mathrm{~K} \alpha, \beta$ and $\delta$ isoforms in the migration of transformed epithelial cells (60), which also illustrates the possibilities in disease treatment of drugs directed against only one of these isoforms.

An interesting protein of the PKC family is PKC $\theta$, which has extensively been studied in relation to inflammatory diseases, such as asthma, arthritis, inflammatory bowel disease, multiple sclerosis, and allograft rejection (61). The present findings indicate that, when using a PKC $\theta$ inhibitor as an anti-inflammatory drug, one has to be aware of possible prothrombotic side-effects due to increased platelet activation. On the other hand, in cancer, $\mathrm{PKC} \alpha$ is already a used target for the drug aprinocarsen (an antisense oligonucleotide) in the treatment of specific neoplastic conditions $(62,63)$. The findings in this thesis suggest that this drug may cause platelet inhibition and suppress thrombus formation. Since arterial thrombosis is a known companion of several cancers and platelets can actively support tumor growth, it is conceivable that the curing effect of aprinocarsen in part occurs via platelet inhibition. However, this needs to be investigated in more detail.

Given the partial thrombo-protective effect of current antiplatelet medication and the significant risk of bleeding complications, it will still be important to test new (high affinity) inhibitors, such as those mentioned above, as future platelet-based antithrombotic drugs. Unfortunately, at these days, phase 2 and 3 clinical trials for new, selective therapeutics are often not performed, because the new drugs need to perform better than standard used drugs or combinations of drugs, resulting in a negative cost-effectiveness ratio. Interestingly, the roads leading to inflammation or cancer sometimes also lead to thrombus formation. Hence, a promising escape from this pitfall is to direct the medical research for antithrombotics towards testing of the drugs directed against platelet signaling molecules, such as those discussed above, that are in current use for anticancer or anti-inflammatory therapy. Such research is highly needed, in particular for patients who suffer from bleeding complications during the current antiplatelet therapy and vice versa for patients with cancer who suffer from thrombotic complications.

\section{References}

1 Farndale RW, Sixma JJ, Barnes MJ, \& de Groot PG. The role of collagen in thrombosis and hemostasis. J Thromb Haemost 2, 561-73 (2004).

2 Nieswandt B \& Watson SP. Platelet-collagen interaction: is GPVI the central receptor? Blood 102, 449-61 (2003).

3 Munnix IC, Kuijpers MJ, Auger J, Thomassen CM, Panizzi P, van Zandvoort MA, Rosing J, Bock PE, Watson SP, \& Heemskerk JW. Segregation of platelet aggregatory and procoagulant microdomains in thrombus formation: regulation by transient integrin activation. Arterioscler Thromb Vasc Biol 27, 2484-90 (2007). 
Pugh N, Simpson AM, Smethurst PA, de Groot PG, Raynal N, \& Farndale RW. Synergism between platelet collagen receptors defined using receptor-specific collagen-mimetic peptide substrata in flowing blood. Blood 115, 5069-79 (2010).

5 Atkinson BT, Jarvis GE, \& Watson SP. Activation of GPVI by collagen is regulated by $\alpha_{2} \beta_{1}$ and secondary mediators. J Thromb Haemost 1, 1278-87 (2003).

6 Chen $\mathrm{H} \&$ Kahn ML. Reciprocal signaling by integrin and nonintegrin receptors during collagen activation of platelets. Mol Cell Biol 23, 4764-77 (2003).

$7 \quad$ Siljander PR, Munnix IC, Smethurst PA, Deckmyn H, Lindhout T, Ouwehand WH, Farndale RW, \& Heemskerk JW. Platelet receptor interplay regulates collagen-induced thrombus formation in flowing human blood. Blood 103, 1333-41 (2004).

8 Asselin J, Knight CG, Farndale RW, Barnes MJ, \& Watson SP. Monomeric (glycine-proline-hy droxyproline)1o repeat sequence is a partial agonist of the platelet collagen receptor glycoprotein VI. Biochem J 339, 413-8 (1999).

9 Emsley J, Knight CG, Farndale RW, \& Barnes MJ. Structure of the integrin $\alpha_{2} \beta_{1}$-binding collagen peptide. J Mol Biol 335, 1019-28 (2004).

10 Inoue O, Suzuki-Inoue K, Dean WL, Frampton J, \& Watson SP. Integrin $\alpha_{2} \beta_{1}$ mediates outside-in regulation of platelet spreading on collagen through activation of Src kinases and PLC $\gamma_{2}$. J Cell Biol 160, 769-80 (2003).

11 Consonni A, Cipolla L, Guidetti G, Canobbio I, Ciraolo E, Hirsch E, Falasca M, Okigaki M, Balduini $C_{1}$ \& Torti M. Role and regulation of phosphatidylinositol 3-kinase $\beta$ in platelet integrin $\alpha_{2} \beta_{1}$ signaling. Blood 119, 847-56 (2012).

12 Hirsch E, Bosco O, Tropel P, Laffargue M, Calvez R, Altruda F, Wymann M, \& Montrucchio G. Resistance to thromboembolism in $\mathrm{Pl}_{3} \mathrm{~K} \gamma$-deficient mice. FASEB J 15, 2019-21 (2001).

13 Senis $Y A$, Atkinson BT, Pearce AC, Wonerow $P$, Auger JM, Okkenhaug K, Pearce W, Vigorito $E$, Vanhaesebroeck B, Turner M, \& Watson SP. Role of the p110 $\mathrm{PI}$ 3-kinase in integrin and ITAM receptor signalling in platelets. Platelets 16, 191-202 (2005).

14 Lu PJ, Hsu AL, Wang DS, \& Chen CS. Phosphatidylinositol 3,4,5-trisphosphate triggers platelet aggregation by activating $\mathrm{Ca}^{2+}$ influx. Biochemistry 37, 9776-83 (1998).

15 Pasquet JM, Quek L, Stevens C, Bobe R, Huber M, Duronio V, Krystal G, \& Watson SP. Phosphatidylinositol 3,4,5-trisphosphate regulates $\mathrm{Ca}^{2+}$ entry via $\mathrm{Btk}$ in platelets and megakaryocytes without increasing phospholipase C activity. EMBO J 19, 2793-802 (2000).

16 Lova P, Paganini S, Hirsch E, Barberis L, Wymann M, Sinigaglia F, Balduini C, \& Torti M. A selective role for phosphatidylinositol 3,4,5-trisphosphate in the Gi-dependent activation of platelet Rap1B. J Biol Chem 278, 131-8 (2003).

17 Woulfe $D$, Jiang $H$, Mortensen R, Yang J, \& Brass LF. Activation of Rap1B by Gi family members in platelets. J Biol Chem 277, 23382-90 (2002).

18 Konopatskaya O, Matthews SA, Harper MT, Gilio K, Cosemans JM, Williams CM, Navarro MN, Carter DA, Heemskerk JW, Leitges M, Cantrell D, \& Poole AW. Protein kinase C mediates platelet secretion and thrombus formation through protein kinase D2. Blood 118, 416-24 (2011).

19 Chari R, Getz T, Nagy B, Jr., Bhavaraju K, Mao Y, Bynagari YS, Murugappan S, Nakayama K, \& Kunapuli SP. Protein kinase $C \delta$ differentially regulates platelet functional responses. Arterioscler Thromb Vasc Biol 29, 699-705 (2009).

20 Pula G, Schuh K, Nakayama K, Nakayama KI, Walter U, \& Poole AW. PKC $\delta$ regulates collageninduced platelet aggregation through inhibition of VASP-mediated filopodia formation. Blood 108, 4035-44 (2006).

21 Strehl A, Munnix IC, Kuijpers MJ, van der Meijden PE, Cosemans JM, Feijge MA, Nieswandt B, \& Heemskerk JW. Dual role of platelet protein kinase $C$ in thrombus formation: stimulation of proaggregatory and suppression of procoagulant activity in platelets. J Biol Chem 282, 7046-55 (2007).

22 Nagy B, Jr., Bhavaraju K, Getz T, Bynagari YS, Kim S, \& Kunapuli SP. Impaired activation of platelets lacking protein kinase $C \theta$ isoform. Blood 113, 2557-67 (2009).

23 Cohen S, Braiman A, Shubinsky G, Ohayon A, Altman A, \& Isakov N. PKC $\theta$ is required for hemostasis and positive regulation of thrombin-induced platelet aggregation and $\alpha$-granule secretion. Biochem Biophys Res Commun 385, 22-7 (2009). 
Mangin P, Yap CL, Nonne C, Sturgeon SA, Goncalves I, Yuan Y, Schoenwaelder SM, Wright CE, Lanza F, \& Jackson SP. Thrombin overcomes the thrombosis defect associated with platelet GPVI/FcRy deficiency. Blood 107, 4346-53 (2006).

25 Varga-Szabo D, Braun A, Kleinschnitz C, Bender M, Pleines I, Pham M, Renne T, Stoll G, \& Nieswandt B. The calcium sensor STIM1 is an essential mediator of arterial thrombosis and ischemic brain infarction. J Exp Med 205, 1583-91 (2008).

26 Braun A, Varga-Szabo D, Kleinschnitz C, Pleines I, Bender M, Austinat M, Bosl M, Stoll G, \& Nieswandt B. Orai1 (CRACM1) is the platelet SOC channel and essential for pathological thrombus formation. Blood 113, 2056-63 (2009).

27 Bergmeier W, Oh-Hora M, McCarl CA, Roden RC, Bray PF, \& Feske S. R93W mutation in Orai1 causes impaired calcium influx in platelets. Blood 113, 675-8 (2009).

28 Zhang SL, Yu Y, Roos J, Kozak JA, Deerinck TJ, Ellisman MH, Stauderman KA, \& Cahalan MD. STIM1 is a $\mathrm{Ca}^{2+}$ sensor that activates CRAC channels and migrates from the $\mathrm{Ca}^{2+}$ store to the plasma membrane. Nature 437, 902-5 (2005).

29 Merritt JE, Armstrong WP, Benham CD, Hallam TJ, Jacob R, Jaxa-Chamiec A, Leigh BK, McCarthy $\mathrm{SA}$, Moores KE, \& Rink TJ. SKF96365, a novel inhibitor of receptor-mediated calcium entry. Biochem J 271, 515-22 (1990).

30 Harper MT \& Poole AW. Store-operated calcium entry and non-capacitative calcium entry have distinct roles in thrombin-induced calcium signalling in human platelets. Cell Calcium 50, 351-8 (2011).

31 Rosado JA, Brownlow SL, \& Sage SO. Endogenously expressed Trp1 is involved in store-mediated $\mathrm{Ca}^{2+}$ entry by conformational coupling in human platelets. J Biol Chem 277, 42157-63 (2002).

32 Varga-Szabo D, Authi KS, Braun A, Bender M, Ambily A, Hassock SR, Gudermann T, Dietrich A, \& Nieswandt $\mathrm{B}$. Store-operated $\mathrm{Ca}^{2+}$ entry in platelets occurs independently of transient receptor potential channel 1. Pflügers Arch 457, 377-87 (2008).

33 Hassock SR, Zhu MX, Trost C, Flockerzi V, \& Authi KS. Expression and role of TRPC proteins in human platelets: evidence that TRPC6 forms the store-independent calcium entry channel. Blood 100, 2801-11 (2002).

34 Zhang SL, Kozak JA, Jiang W, Yeromin AV, Chen J, Yu Y, Penna A, Shen W, Chi V, \& Cahalan MD. Store-dependent and -independent modes regulating $\mathrm{Ca}^{2+}$ release-activated $\mathrm{Ca}^{2+}$ channel activity of human Orai1 and Orai3. J Biol Chem 283, 17662-71 (2008).

Ramanathan G, Gupta S, Thielmann I, Pleines I, Varga-Szabo D, May F, Mannhalter C, Dietrich A, Nieswandt B, \& Braun A. Defective diacylglycerol-induced $\mathrm{Ca}^{2+}$ entry but normal agonist-induced activation responses in TRPC6 deficient mouse platelets. J Thromb Haemost 10, 419-29 (2012). Nieuwland R, van Willigen G, \& Akkerman JW. Different pathways for control of $\mathrm{Na}^{+} / \mathrm{H}^{+}$exchange via activation of the thrombin receptor. Biochem J 297, 47-52 (1994).

37 Suzuki J, Umeda M, Sims PJ, \& Nagata S. Calcium-dependent phospholipid scrambling by TMEM16F. Nature 468, 834-8 (2010).

38 Munnix IC, Harmsma M, Giddings JC, Collins PW, Feijge MA, Comfurius P, Heemskerk JW, \& Bevers EM. Store-mediated calcium entry in the regulation of phosphatidylserine exposure in blood cells from Scott patients. Thromb Haemost 89, 687-95 (2003).

39 Harper MT \& Sage SO. PAR-1-dependent pp6osrc activation is dependent on protein kinase C and increased $\left[\mathrm{Ca}^{2+}\right]$ : evidence that pp6osrc does not regulate PAR-1-dependent $\mathrm{Ca}^{2+}$ entry in human platelets. J Thromb Haemost 4, 2695-703 (2006).

40 Harper MT, Molkentin JD, \& Poole AW. Protein kinase C $\alpha$ enhances sodium-calcium exchange during store-operated calcium entry in mouse platelets. Cell Calcium 48, 333-40 (2010).

41 Harper MT \& Poole AW. Protein kinase $\mathrm{C} \theta$ negatively regulates store-independent $\mathrm{Ca}^{2+}$ entry and phosphatidylserine exposure downstream of glycoprotein VI in platelets. J Biol Chem 285, 1986573 (2010).

Schoenwaelder SM, Yuan Y, Cooray P, Salem HH, \& Jackson SP. Calpain cleavage of focal adhesion proteins regulates the cytoskeletal attachment of integrin $\alpha \mathrm{llb} \beta_{3}$ and the cellular retraction of fibrin clots. J Biol Chem 272, 1694-702 (1997).

43 Pfaff $M$, Du $X, \&$ Ginsberg MH. Calpain cleavage of integrinb cytoplasmic domains. FEBS Lett 460, 17-22 (1999). 
44 Xi X, Bodnar RJ, Li Z, Lam SC, \& Du X. Critical roles for the COOH-terminal NITY and RGT sequences of the integrin $\beta_{3}$ cytoplasmic domain in inside-out and outside-in signaling. J Cell Biol 162, 329-39 (2003).

45 Parekh AB. Mitochondrial regulation of intracellular $\mathrm{Ca}^{2+}$ signaling: more than just simple $\mathrm{Ca}^{2+}$ buffers. News Physiol Sci 18, 252-6 (2003).

46 Jobe SM, Wilson KM, Leo L, Raimondi A, Molkentin JD, Lentz SR, \& Di Paola J. Critical role for the mitochondrial permeability transition pore and cyclophilin $D$ in platelet activation and thrombosis. Blood 111, 1257-65 (2008).

47 Schoenwaelder SM, Yuan Y, Josefsson EC, White MJ, Yao Y, Mason KD, O'Reilly LA, Henley KJ, Ono A, Hsiao S, Willcox A, Roberts AW, Huang DC, Salem HH, Kile BT, \& Jackson SP. Two distinct pathways regulate platelet phosphatidylserine exposure and procoagulant function. Blood 114, 663-6 (2009).

48 Barsukova AG, Bourdette D, \& Forte M. Mitochondrial calcium and its regulation in neurodegeneration induced by oxidative stress. Eur J Neurosci 34, 437-47 (2011).

49 Pinton P, Leo S, Wieckowski MR, Di Benedetto G, \& Rizzuto R. Long-term modulation of mitochondrial $\mathrm{Ca}^{2+}$ signals by protein kinase $\mathrm{C}$ isozymes. J Cell Biol 165, 223-32 (2004).

50 Novo E \& Parola M. Redox mechanisms in hepatic chronic wound healing and fibrogenesis. Fibrogenesis Tissue Repair 1, 5 (2008).

51 Rosado JA, Lopez JJ, Harper AG, Harper MT, Redondo PC, Pariente JA, Sage SO, \& Salido GM. Two pathways for store-mediated calcium entry differentially dependent on the actin cytoskeleton in human platelets. J Biol Chem 279, 29231-5 (2004).

52 van Kruchten R, Braun A, Feijge MA, Kuijpers MJ, Rivera-Galdos R, Kraft P, Stoll G, Kleinschnitz C, Bevers EM, Nieswandt B, \& Heemskerk JW. Antithrombotic potential of blockers of storeoperated calcium channels in platelets. Arterioscler Thromb Vasc Biol 32, 1717-23 (2012).

53 Vogt PK. Drug-resistant phosphatidylinositol 3-kinase: Guidance for the preemptive strike. Cancer Cell 14, 107-8 (2008).

54 Paz-Ares L, Blanco-Aparicio C, Garcia-Carbonero R, \& Carnero A. Inhibiting Pl3K as a therapeutic strategy against cancer. Clin Transl Oncol 11, 572-9 (2009).

55 Bynagari-Settipalli YS, Chari R, Kilpatrick L, \& Kunapuli SP. Protein kinase C - possible therapeutic target to treat cardiovascular diseases. Cardiovasc Hematol Disord Drug Targets 10, 292-308 (2010).

56 Jackson SP \& Schoenwaelder SM. Antiplatelet therapy: in search of the 'magic bullet'. Nat Rev Drug Discov 2, 775-89 (2003).

57 Cosemans JM, Munnix IC, Wetzker R, Heller R, Jackson SP, \& Heemskerk JW. Continuous signal ing via $\mathrm{Pl}_{3} \mathrm{~K}$ isoforms $\beta$ and $\gamma$ is required for platelet ADP receptor function in dynamic thrombus stabilization. Blood 108, 3045-52 (2006).

58 Jackson SP, Schoenwaelder SM, Goncalves I, Nesbitt WS, Yap CL, Wright CE, Kenche V, Anderson KE, Dopheide SM, Yuan Y, Sturgeon SA, Prabaharan H, Thompson PE, Smith GD, Shepherd PR, Daniele N, Kulkarni S, Abbott B, Saylik D, Jones C, Lu L, Giuliano S, Hughan SC, Angus JA, Robertson AD, \& Salem HH. PI 3-kinase p110 $\beta$ : a new target for antithrombotic therapy. Nat Med 11, 507-14 (2005).

59 Jackson SP. Arterial thrombosis-insidious, unpredictable and deadly. Nat Med 17, 1423-36 (2011).

60 De Laurentiis A, Pardo OE, Palamidessi A, Jackson SP, Schoenwaelder SM, Reichmann E, Scita G, \& Arcaro $\mathrm{A}$. The catalytic class $\mathrm{IA} \mathrm{Pl}_{3} \mathrm{~K}$ isoforms play divergent roles in breast cancer cell migration. Cell Signal 23, 529-41 (2011).

61 Boschelli DH. Small molecule inhibitors of PKC $\theta$ as potential antiinflammatory therapeutics. Curr Top Med Chem 9, 640-54 (2009).

62 Hanauske AR, Sundell K, \& Lahn M. The role of protein kinase $\mathrm{C} \alpha$ in cancer and its modulation by the novel PKC $\alpha$-specific inhibitor aprinocarsen. Curr Pharm Des 10, 1923-36 (2004).

63 Lahn M, Sundell K, \& Kohler G. The role of protein kinase C $\alpha$ in hematologic malignancies. Acta Haematol 115, 1-8 (2006). 



\section{Summary}


Platelets are the principal blood cell components implicated in thrombosis and hemostasis. Following vascular injury or activation, platelets form a thrombus at the damaged site, causing the cessation of bleeding. In the pathophysiological process of thrombosis at the site of a ruptured atherosclerotic plaque, uncontrolled thrombus formation can lead to vessel occlusion and result in heart attack or stroke. In platelets, many intracellular signaling pathways have been identified that contribute to platelet activation processes, like integrin activation, secretion, platelet aggregation and procoagulant activity (phosphatidylserine exposure). Unraveling which of these signaling roads lead to thrombus formation was endeavored in this thesis.

Chapter 1 gives a general overview of relevant mechanisms of platelet receptor activation and intracellular signaling pathways, leading to integrin activation, secretion and procoagulant activity. The precise role of the signaling collagen receptor, glycoprotein $\mathrm{VI}$, in collagen-induced thrombus formation is extensively described, but that of the adhesive collagen receptor, integrin $\alpha_{2} \beta_{1}$, is less clear. In the signal transduction pathway induced by glycoprotein $\mathrm{VI}$, phosphoinositide 3 -kinase $\left(\mathrm{PI}_{3} \mathrm{~K}\right.$ ) plays a key role in integrin $\alpha$ llb $\beta_{3}$ activation and platelet aggregation. However, which of the several $\mathrm{Pl}_{3} \mathrm{~K}$ isoforms is responsible for these responses remains to be clarified. This also holds for participation of the small GTPase Rap1b, which operates downstream of $\mathrm{Pl}_{3} \mathrm{~K}$. The main phospholipid substrate of Pl3K, phosphatidylinositol 4,5-bisphosphate, also a substrate for phospholipase $C$, cleaving this into inositol trisphosphate and diacylglycerol. The latter activates members of the family of protein kinase C (PKC), which are implicated in almost all platelet responses. However, which of the various expressed PKC isoforms contributes to which platelet responses during thrombus formation, needs to be investigated. $\mathrm{As}^{2{ }^{2+}}$ is an important second messenger in the different stages of platelet activation, possible regulators of cytosolic $\mathrm{Ca}^{2+}$ mobilization are discussed as well.

To address the question whether collagen-mimetic peptides support flow-dependent thrombus formation, we used in chapter 2 triple-helical peptides consisting of hexapeptide sequences of the recognition site for integrin $\alpha_{2} \beta_{1}$, i.e. peptides with high-affinity (GF/LOGER), intermediate affinity (GLM/SGER), or low-affinity (GAO/SGER) binding. The hexapeptide sequence was incorporated in a backbone of repeated (GPP) ${ }_{n}$ sequences as a neutral control, or of (GPO) $)_{n}$ sequences to provide additional binding sites for glycoprotein VI. These triple-helical peptides were immobilized to glass in the presence or absence of von Willebrand factor to allow platelet interaction with glycoprotein Ib-V-IX. Whole blood perfusion at high shear rate showed that immobilized peptides containing both the high-affinity $\alpha_{2} \beta_{1}$-binding motif GFOGER and the (GPO) $)_{n}$ motif supported platelet aggregation and procoagulant activity, even in the absence of von Willebrand factor. With peptides containing only one of these motifs, co-immobilized von Willebrand factor was needed for thrombus formation. Peptides with intermediate affinity or low-affinity $\alpha_{2} \beta_{1}$-binding motifs formed procoagulant thrombi only if both $(\mathrm{GPO})_{n}$ and von Willebrand factor were present. At a low-shear rate, immobilized peptides with high- or low-affinity $\alpha_{2} \beta_{1}$-binding motifs mediated formation of thrombi with procoagulant platelets only in combination with $(\mathrm{GPO})_{n}$. Taken together, chapter 2 indicates that collagen-mimetic, triple-helical peptides 
effectively and efficiently can take over the functions of native collagens in mediating platelet adhesion and activation via the receptors glycoprotein $\mathrm{VI}$ and $\alpha_{2} \beta_{1}$. Herein, glycoprotein VI exerts a signaling rather than adhesive function, whereas $\alpha_{2} \beta_{1}$ and von Willebrand factor determine the adhesion of platelets under flow conditions.

In chapter 3 , we identified $\mathrm{Pl}_{3} \mathrm{~K} \alpha$ and $\beta$ as the main $\mathrm{Pl}_{3} \mathrm{~K}$ isoforms for full phospholipase $C$ activation, inositol trisphosphate formation and further signaling evoked by glycoprotein VI. We could not confirm a role of these isoforms in $\mathrm{Ca}^{2+}$ entry from the extracellular medium. In platelets from mice lacking p85 (i.e. a main regulatory subunit of $\mathrm{Pl}_{3} \mathrm{~K} \alpha / \beta$ ), collagen-induced $\mathrm{Ca}^{2+}$ signaling and thrombus formation were severely impaired. Applying a pharmacological approach with high affinity inhibitors of $\mathrm{Pl}_{3} \mathrm{~K} \alpha$ (PIK-75) or $\mathrm{Pl} 3 \mathrm{~K} \beta$ (TGX221), a functional non-redundancy of these isoforms in glycoprotein VI-induced platelet activation was found, since the inhibitors together did not have an additional effect on Akt phosphorylation, secretion, $\mathrm{Ca}^{2+}$ signaling, procoagulant activity, and thrombus formation. Furthermore, chapter 3 provides evidence of a direct role for glycoprotein VI in Rapıb activation in both human and mouse platelets. This direct contribution could be attributed to activation of the $\mathrm{Pl}_{3} \mathrm{~K} \alpha$ and $\beta$ isoforms. Because $\mathrm{Pl}_{3} \mathrm{~K}$ inhibitors still suppressed integrin $\alpha$ llb $\beta_{3}$ activation and platelet aggregation in Rap $1 b^{-1-}$ platelets, we concluded that there also exists a $\mathrm{Pl}_{3} \mathrm{~K}$-mediated, Rapıb-independent pathway of integrin activation. This pathway may involve $\mathrm{Pl}_{3} \mathrm{~K} \alpha / \beta$-dependent stimulation of phospholipase $\mathrm{C}_{\gamma 2}$, which possibly contributes to $\mathrm{Ca}^{2+}$ - and PKC-mediated activation of integrin $\alpha$ llb $\beta_{3}$. Together, these findings provide a new mechanistic explanation for the antithrombotic effect of $\mathrm{Pl}_{3} \mathrm{~K}$ inhibition and make $\mathrm{Pl}_{3} \mathrm{~K} \alpha / \beta$ interesting targets for anti-platelet therapy.

By applying both a genetic and pharmacological approach, in chapters 4 and 5 we identified the roles of the different PKC isoforms, i.e. the conventional (PKC $\alpha$ and $\beta$ ) and novel (PKC $\delta$ and $\theta$ ) isoforms, in the process of thrombus formation. Chapter 4 reveals a key role for $\mathrm{PKC} \alpha$ in regulating granule biogenesis and exocytosis. In addition, it is shown that glycoprotein VI- and thrombin-induced secretion of $\alpha$-granules, integrin $\alpha$ llb $\beta_{3}$ activation, and platelet aggregation are positively regulated by PKC $\alpha$. On the other hand, as presented in chapter 4 , PKC $\alpha$ does not affect platelet adhesion and spreading on collagen. The inhibitory effects of PKC $\alpha$ ablation on granule secretion and thrombus formation favor the thought of using PKC $\alpha$ as a drug target for antithrombotic therapy. In chapter 5, the comparison of mouse platelets lacking different isoforms of $\mathrm{PKC}$, by measuring thrombus formation under whole blood flow conditions, reveals distinct, non-redundant roles of the isoforms. It is concluded that the conventional PKCs (PKC $\alpha$ and $\beta$ ) promote, and the novel PKCs (PKC $\delta$ and $\theta$ ) inhibit thrombus formation on collagen. Considering $\mathrm{Ca}^{2+}$ signaling and procoagulant activity, these responses were both diminished in platelets from $\mathrm{PKCa}^{-\gamma_{-}}$and $\mathrm{PKC}^{-1-}$ mice. These findings are supported by pharmacological studies with human platelets, using Gö6976 and a PKC $\beta$ inhibitor, respectively. On the contrary, platelets from mice deficient in $\mathrm{PKC} \theta$, and to a lesser extent $\mathrm{PKC} \delta$, were increased in collagen-dependent granule secretion and thrombus formation. This was supported by enhanced $\mathrm{Ca}^{2+}$ responses and increased phosphatidylserine exposure of $\mathrm{PKC}^{-/}$platelets. Comparable results were obtained with human platelets pretreated with a PKC $\theta$ inhibitor or with the PKC $\delta$ inhibitor 
rottlerin. Regarding chapters 4 and 5, we propose that the conventional $P K C \alpha / \beta$ isoforms are essential for collagen-induced granule secretion, phosphatidylserine exposure, and thrombus formation, while especially PKC $\theta$ negatively regulates these processes.

We investigated in chapter 6 whether the $\mathrm{Ca}^{2+}$-sensing proteins STIM1 and 2 and the $\mathrm{Ca}^{2+}$-selective channel Orai1 are key $\mathrm{Ca}^{2+}$ regulatory components in platelet phosphatidylserine exposure and thrombus formation upon stimulation of the collagen and thrombin receptors. The studies were carried out using chimeric mice with platelets deficient in STIM, STIM2, or Orai1. It was demonstrated that deficiency in either STIM1 (but not STIM2) or Orai1 greatly reduced glycoprotein $\mathrm{VI}$-induced $\mathrm{Ca}^{2+}$ signaling, phosphatidylserine exposure, and thrombus formation under flow conditions in the absence of coagulation. In the presence of high thrombin concentrations, these platelet responses tended to normalize. These findings jointly suggest that the glycoprotein VI-induced $\mathrm{Ca}^{2+}$ entry pathway mediated by $\mathrm{STIM}_{1}$ and Orai1 becomes redundant in the regulation of thrombus formation under conditions where thrombin acts as co-agonist.

In chapter 7, the signaling processes were studied by which phosphatidylserineexposing platelets inactivate or close integrin $\alpha$ llb $\beta_{3}$. In particular, we examined the possible roles in integrin closure of elevated $\mathrm{Ca}^{2+}$, Src family kinases, mitochondrial permeability transition pore formation, and calpain-dependent protein cleavage. The data showed that both $\mathrm{Ca}^{2+}$ elevation (with likely a role of mitochondrial dysfunction) and calpain activity are involved in the control of integrin closure, although also the process of phosphatidylserine exposure itself may contribute to integrin closure. Key findings of this thesis as well as their possible clinical significance, in relation to the current literature, are discussed in chapter 8. It is argued that thrombus formation is delicately regulated by various cooperating signaling pathways with sometimes redundant, sometimes non-redundant roles of specific isoforms of $\mathrm{Pl}_{3} \mathrm{~K}, \mathrm{PKC}$ and $\mathrm{Ca}^{2+}$-regulating proteins in platelets. Overall, the studies in this thesis help to demystify the current uncertainty of precise signaling pathways towards thrombus formation. In anti-thrombotic treatment the present antiplatelet drugs are only limitedly effective and have as side effect an increased risk of bleeding. To develop personalized targetspecific drugs, which have lower side effects, more precise knowledge of the process of thrombus formation and platelet signaling is essential. The present insight, described in this thesis, hence may contribute to future improvements in the treatment of arterial thrombosis. 
Samenvatting 
Als kleinste cellulaire bloedcomponent hebben bloedplaatjes een centrale rol in de processen van trombose en hemostase. Wanneer een bloedvat beschadigd wordt, vormen geactiveerde bloedplaatjes aldaar een trombus waardoor de bloeding gestelpt wordt. De pathofysiologische tegenhanger van hemostase is trombose. Hierbij ontstaat ongecontroleerde trombusvorming, bijvoorbeeld ten gevolge van een ruptuur van een aterosclerotische plaque, mogelijk leidend tot bloedvatafsluiting resulterend in een hartinfarct of een beroerte. In bloedplaatjes zijn tot dusver vele intracellulaire signaleringspaden geïdentificeerd, die bijdragen aan de verschillende activatieprocessen van deze cellen, waaronder integrine-activatie, secretie, plaatjesaggregatie en stollingsbevorderende activiteit (oppervlakte-expositie van fosfatidylserine, PS). Het doel van deze thesis is meer inzicht te krijgen in het belang van die signaleringspaden leidend tot trombusvorming.

Hoofdstuk 1 geeft een algemeen overzicht van de relevante mechanismen van receptoractivatie en intracellulaire signaleringspaden in bloedplaatjes, betrokken bij integrineactivatie, secretie en procoagulante activiteit. De exacte rol van de signalerende collageenreceptor, glycoproteïne VI, in het proces van collageen-geïnduceerde trombusvorming was eerder al beschreven. Die van de adhesieve collageenreceptor integrine $\alpha_{2} \beta_{1}$ daarentegen is minder duidelijk. In het signaaltransductiepad geïnduceerd door glycoproteïne VI spelen eiwitten van de familie van fosfoïnositide 3 -kinase $\left(\mathrm{Pl}_{3} \mathrm{~K}\right.$ ) een centrale rol bij de activatie van integrine $\alpha \mathrm{llb} \beta_{3}$ en de daaropvolgende bloedplaatjesaggregatie. Maar welke van de verschillende $\mathrm{Pl}_{3} \mathrm{~K}$ isovormen verantwoordelijk zijn voor deze activatieresponsen dient nog onderzocht te worden. Dit geldt ook voor de bijdrage van het onder $\mathrm{Pl}_{3} \mathrm{~K}$ functionerende regeleiwit, de GTPase Rap1b. Het belangrijkste fosfolipidensubstraat van $\mathrm{Pl}_{3} \mathrm{~K}$, fosfatidylinositol 4,5-bifosfaat, is ook een substraat voor fosfolipase C, die dit fosfolipide knipt in diacylglycerol en inositol-trifosfaat. Diacylglycerol activeert eiwitten van de proteïne kinase $C$ (PKC) familie, die betrokken zijn bij zowat alle activatieresponsen in bloedplaatjes. Maar welke van de verschillende PKC isovormen betrokken zijn bij die verschillende responsen tijdens trombusvorming, dient nog uitgezocht te worden. Aangezien het ion $\mathrm{Ca}^{2+}$ bekend staat als een belangrijke intracellulaire boodschapper bij de verschillende fasen van bloedplaatjesactivatie, worden in dit hoofdstuk ook de mogelijke regulatoren van cytosolaire $\mathrm{Ca}^{2+}$-mobilisatie besproken.

Voor het oplossen van de vraag of collageen-nabootsende peptiden trombusvorming in stromend bloed ondersteunen, zijn in hoofdstuk 2 enige triple-helix peptiden gebruikt bestaande uit hexapeptidesequenties waaraan integrine $\alpha_{2} \beta_{1}$ kan binden, dat wil zeggen peptiden met een hoge (GF/LOGER), intermediaire (GLM/SGER) of lage (GAO/SGER) affiniteit voor dit integrine. Deze hexapeptidesequenties zijn ingebouwd in een structuur van herhaalde $(\mathrm{GPP})_{\mathrm{n}}$-sequenties als controle-eiwit, of van (GPO) $)_{n}$-sequenties om een additionele bindingsplaats voor glycoproteïne VI te creëren. De triple-helix peptiden werden op glas geïmmobiliseerd in de aan- of afwezigheid van von Willebrand factor (vWF), waardoor al dan niet interactie van bloedplaatjes met glycoproteïne Ib-V-IX mogelijk werd. Bloeddoorstroming bij hoge afschuifsnelheden over deze eitwitoppervlakken toonde aan dat geïmmobiliseerde peptiden, die zowel het hoge-affiniteit $\alpha_{2} \beta_{1}$-bindingsmotief GFOGER als de sequentie (GPO) n bevatten, de aggregatie en procoagulante activiteit van 
bloedplaatjes kunnen opwekken, zelfs in de afwezigheid van vWF. Peptiden die enkel een van beide motieven bevatten dienden gecoïmmobiliseerd te worden met vWF om een trombus te kunnen vormen. Peptiden met intermediaire of lage affiniteitsbinding voor $\alpha_{2} \beta_{1}$ stimuleerden de vorming van procoagulante trombi alleen in de aanwezigheid van (GPO) ${ }_{n}$ sequenties en vWF. Bij lage afschuifsnelheden stimuleerden geïmmobiliseerde peptiden met hoge- of lage-affiniteit $\alpha_{2} \beta_{1}$-bindende motief, enkel in combinatie met (GPO) ${ }_{n}$ de vorming van trombi met procoagulante bloedplaatjes. Samengevat toont hoofdstuk 2 aan dat collageen-nabootsende, triple-helix peptiden effectief en efficiënt de functie kunnen overnemen van collageen in bloedplaatjesadhesie en -activatie via glycoproteïne VI en integrine $\alpha_{2} \beta_{1}$. Hieruit blijkt dat glycoproteïne $\mathrm{VI}$ eerder een signalerende dan een adhesieve functie heeft, terwijl integrine $\alpha_{2} \beta_{1}$ en de vWF-receptor glycoproteïne Ib-V-IX zorgen voor adhesie van bloedplaatjes onder stromingsomstandigheden.

In hoofdstuk 3 zijn $\mathrm{Pl}_{3} \mathrm{~K} \alpha$ en $\beta$ geïdentificeerd als de belangrijkste $\mathrm{Pl}_{3} \mathrm{~K}$ isovormen noodzakelijk voor activatie van fosfolipase $C$, vorming van inositol-trifosfaat en daaropvolgende signalering opgewekt door glycoproteïne VI. Een mogelijke bijdrage van deze isovormen in de $\mathrm{Ca}^{2+}$-instroom vanuit het extracellulaire milieu kon niet worden bevestigd. In bloedplaatjes van muizen zonder het eiwit $\mathrm{p} 85 \alpha$ (dé regulatoire component van $\mathrm{Pl}_{3} \mathrm{~K} \alpha / \beta$ ) bleken de collageen-geïnduceerde $\mathrm{Ca}^{2+}$-signalering en trombusvorming ernstig verstoord. In een farmacologische benadering met selectieve, hoge affiniteits-remmers van $\mathrm{PI}_{3} \mathrm{~K} \alpha$ (PIK-75) of $\mathrm{Pl}_{3} \mathrm{~K} \beta$ (TGX-221) kon worden aangetoond dat de gelijktijdige werking van beide isovormen nodig is voor bloedplaatjesactivatie door glycoproteïne VI. Bewijs hiervoor was ondermeer dat de combinatie van deze remmers geen additioneel effect had op de processen van Akt fosforylatie, $\mathrm{Ca}^{2+}$-signalering, plaatjessecretie, procoagulante activiteit en trombusvorming. Bovendien laat hoofdstuk 3 zien dat er een directe rol is van glycoproteïne VI in de activatie van Rapıb, zowel bij humane plaatjes als bij muizenplaatjes. Deze bijdrage loopt via de isovormen $\mathrm{Pl}_{3} \mathrm{~K} \alpha$ en $\beta$. Omdat ook in $\mathrm{Rap}_{1} \mathrm{~b}^{-/}$muizen remming van $\mathrm{Pl}_{3} \mathrm{~K}$ nog steeds van invloed was op de $\alpha$ llb $\beta_{3}$-activatie en bloedplaatjesaggregatie, kan geconcludeerd worden dat er een alternatieve Rapıb-onafhankelijke signaleringsroute voor integrine-activatie bestaat middels $\mathrm{Pl}_{3} \mathrm{~K}$. Deze route zou kunnen verlopen door middel van $\mathrm{Pl}_{3} \mathrm{~K} \alpha / \beta$-afhankelijke stimulatie van fosfolipase $\mathrm{C}_{2}$, met als gevolg een $\mathrm{Ca}^{2+} / \mathrm{PKC}$ gemediëerde activatie van integrine $\alpha \mathrm{llb} \beta_{3}$. Recapitulerend geven bovenstaande bevindingen een nieuwe mechanistische verklaring voor het antitrombotische effect van $\mathrm{Pl} 3 \mathrm{~K} \alpha / \beta$ remming, waarmee deze eiwitten mogelijk een effectief doelwit zijn voor antibloedplaatjestherapie.

Door genetische en farmacologische benaderingen werden in de hoofdstukken 4 en 5 de functies van de verschillende isovormen van PKC geïdentificeerd in het proces van trombusvorming. Dit betreft de conventionele isovormen PKC $\alpha$ en $\beta$, en de 'nieuwe' isovormen PKC $\delta$ en $\theta$. Hoofdstuk 4 onthult een sleutelrol voor de isovorm PKC $\alpha$ in de biogenese van opslag-granula en in de secretie van de inhoud van deze granula. Aangetoond werd dat onder invloed van glycoproteïne VI en trombine de secretie van $\alpha$-granula, evenals de integrine $\alpha$ llb $\beta_{3}$-activatie en plaatjesaggregatie positief gereguleerd worden door PKC $\alpha$. Evenwel heeft PKC $\alpha$ geen invloed op bloedplaatjesadhesie en -spreiding over een collageenop- 
pervlak. De sterk stimulerende bijdrage van PKC $\alpha$ op bloedplatjesactivatie en trombusvorming ondersteunen het idee om te onderzoeken of PKC $\alpha$ een goed medicatiedoelwit is voor anti-trombotische therapie. In hoofdstuk 5 zijn muizenbloedplaatjes met een defecte expressie van één van de verschillende PKC isovormen met elkaar vergeleken door meting van de trombusvorming in stromend bloed. Op grond van de resultaten kan worden vastgesteld dat de conventionele en nieuwe isovormen functioneel niet uitwisselbaar zijn. De conventionele PKCs (PKC $\alpha$ en $\beta$ ) bevorderen de trombusvorming op collageen, terwijl de nieuwe PKCs (PKC $\delta$ and $\theta$ ) dit proces onderdrukken. Voorts waren de $\mathrm{Ca}^{2+}$-signalering en procoagulante activiteit gereduceerd in bloedplaatjes van muizen deficiënt in PKC $\alpha$ of PKC $\beta$. Deze bevindingen werden ondersteund door farmacologische studies met humane bloedplaatjes en remmers van deze isovormen (Gö6976 en PKC $\beta$ remmer). Anderzijds werd een toename geconstateerd van de granula-secretie en de trombusvorming bij muizen met een deficiëntie in $\mathrm{PKC} \theta$ of, in mindere mate, $\mathrm{PKC} \delta$. In overeenstemming hiermee waren de $\mathrm{Ca}^{2+}$-responsen en procoagulante activiteit toegenomen bij $\mathrm{PKC}^{-{ }^{-}}$. Vergelijkbare resultaten weren verkregen met humane bloedplaatjes behandeld met een PKC $\theta$ remmer of met de PKC $\delta$ remmer rottlerin. Op basis van de bevindingen in hoofdstukken 4 en 5 is gepostuleerd dat de conventionele $\mathrm{PKC} \alpha / \beta$ isovormen essentieel zijn voor collageengeïnduceerde granula-secretie, procoagulante activiteit (PS-expositie) en trombusvorming, terwijl vooral PKC $\theta$ deze processen negatief reguleert.

In hoofdstuk 6 is onderzocht of de $\mathrm{Ca}^{2+}$-gevoelige eiwitten STIM1 en STIM2 en het $\mathrm{Ca}^{2+}$ selectieve plasmamembraan-kanaal Oraiı een sleutelrol kunnen vervullen bij PS-expositie en trombusvorming bij bloedplaatjesstimulatie met collageen en trombine. Deze studies werden uitgevoerd met chimere muizen met bloedplaatjes die deficiënt waren in STIM1, $\mathrm{STIM}_{2}$ of Orai1. Aangetoond is dat deficiëntie in ofwel STIM1 (doch niet STIM2) ofwel Orai1 leidt tot een sterk verminderde glycoproteïne Vl-geïnduceerde $\mathrm{Ca}^{2+}$-signalering en trombusvorming in stromend bloed onder condities waarbij trombine geïnactiveerd is. Echter bij hoge trombinevorming (en bloedstolling) bleken deze responsen van bloedplaatjes terug normaal. Deze bevindingen wijzen op een compensatoire rol van trombine als 'backup' van de glycoproteïne VI-geïnduceerde $\mathrm{Ca}^{2+}{ }^{2+}$ instroom via Oraiı/STIM1 bij de trombusvorming.

Hoofdstuk 7 beschrijft onderzoek naar signaleringsprocessen die verantwoordelijk zijn voor de sluiting van geactiveerde integrine $\alpha$ llb $\beta_{3}$ in PS-exposerende bloedplaatjes. In het bijzonder werd de rol van een verhoogde $\mathrm{Ca}^{2+}$ concentratie bestudeerd als mogelijke regulator voor integrine-sluiting, maar daarnaast ook de mogelijke bijdragen van Src proteïne kinasen, vorming van de mitochondriële permeabiliteitstransitieporie en van het $\mathrm{Ca}^{2+}$ afhankelijk cellulaire protease calpaïne. De data tonen aan, dat zowel een blijvende verhoging van intracellulair $\mathrm{Ca}^{2+}$ (met vermoedelijk een rol van mitochondriële permeabiliteit) als de protease-activiteit van calpaïne betrokken zijn bij de controle van integrine-sluiting. Daarnaast is niet uitgesloten dat ook het proces van PS-expositie zelf bijdraagt aan het sluiten van de integrines.

De belangrijkste bevindingen van dit proefschrift en de mogelijke klinische relevantie daarvan worden in relatie tot de recente literatuur besproken in hoofdstuk 8 . Beargumenteerd wordt dat trombusvorming een delicaat gereguleerd proces is, waarbij verschillende 
signaleringspaden al dan niet samenwerken of overlappen, en waarbij de verschillende isovormen van $\mathrm{Pl}_{3} \mathrm{~K}, \mathrm{PKC}$ en STIM/Orai soms vervangbaar, soms complementair en soms tegengesteld zijn in werking. De huidige anti-trombotische medicatie gericht op bloedplaatjes is maar ten dele effectief, en heeft als belangrijke complicatie een verhoogd risico op bloedingen. Om een betere gepersonaliseerde medicatie tegen bloedplaatjes te ontwikkelen met minder bijwerkingen, is een goede kennis essentieel van het complexe proces van trombusvorming en de bloedplaatjesactivatie. De nieuwe inzichten beschreven in dit proefschrift kunnen mogelijk helpen om te komen tot een verbeterde behandeling van arteriële trombose. 

Dankwoord 
Promoveren is net als trombusvorming en het leven zelf, een resultaat van een complex samenspel van keuzes en de gevolgen daarvan, verwezenlijkingen op basis van de hierin gestoken energie en tijd, en de steun en hulp van zo velen die je op dit ervaringspad kruisen. Daarom wil ik allen die op een of andere wijze een bijdrage hebben geleverd aan de totstandkoming van dit proefschrift hiervoor bedanken.

\section{Mijn promotieteam}

Allereerst wil ik graag mijn promotor Johan Heemskerk bedanken voor de kansen die hij voor mij gecreëerd heeft. Dit begon al met een uitzonderlijke wijze van mijn aanstelling toen de langverwachte geldstroom voor mijn project op het laatste werd afgeblazen. Verder heb ik genoten van alle buitenlandse ervaringen en contacten die ik tijdens mijn jaren binnen de plaatjesgroep heb kunnen opdoen. Ook de urenlange gesprekken over mogelijke hypothesen en de discussies die daarbij horen, hebben een belangrijke bijdrage gehad aan mijn inzichten en ontwikkeling zowel binnen het kader van het onderzoek als daarbuiten. Hetgeen mij altijd erg motiveerde was uw betrokkenheid, inbreng en ondersteuning bij het testen van onze hypothesen. Ik ben tevreden dat we samen in staat geweest zijn dit promotieonderzoek tot een passend eindresultaat te brengen.

Judith, toen ik startte bij de plaatjesgroep was jij mijn kamergenoot en al bijna aan het einde van jouw promotietraject. De door jou gedeelde ervaring was in meerdere opzichten vaak erg bruikbaar. Onze samenwerking in Bristol was ook iedere keer erg gezellig, doch vermoeiend, maar telkens met veel succes. Nu ben je verder vitgegroeid tot een sterke, succesvolle en veelzijdige onderzoekster. Als copromotor faciliteerde je het efficiënte verloop van het afronden van mijn proefschrift, bedankt hiervoor. Ik wens je veel succes met de verdere uitbouw van jouw toekomst als universitair docent.

\section{Science abroad and succesfull collaborations}

I would like to thank Prof. Dr. Steve Watson, Prof. Dr. Alastair Poole, Prof. Dr. Bernhard Nieswandt, and their labmembers for welcoming me into their labs and all the help with the experiments and discussing several hypotheses. These visits have been a great learning experience for me, both scientific and personal. Also, I am greatly thankful for our longterm collaboration which has resulted in several publications. Furthermore, I would like to thank Prof. Dr. Richard Farndale, Prof. Dr. Shawn Jackson, Dr. Stewart Sage, and Dr. Shawn Jobe for the cooperation leading to several individual papers.

\section{Mijn paranimfjes}

Imke, vanaf onze eerste (werkgerelateerde) uitstap naar het buitenland zeer snel na de start van mijn promotietraject klikte het goed tussen ons. Jij leek wel mijn oudere 'zus', zo aan het einde van jouw promotietijd. Met jouw samenwerken was altijd een plezier! Ik ben heel blij dat we nog steeds contact hebben ondanks dat je na je post-doc periode de weg van de klinische chemie bent ingeslagen. Jij was ook de eerste om aan te geven dat ik ondanks mijn wetenschappelijke interesse toch eerder gemaakt ben voor een vervolgcarrièrre in de gezondheidszorg. Nu ik deze richting ben ingeslagen blijkt ook dat ik er zeer op mijn plaats zit. 
Ook tijdens de afrondingsfase van mijn proefschrift, terwijl jij reeds in de helft van je droomopleiding zat, gaf je altijd goede tips en heeft je kordate en eerlijke raad regelmatig nut gehad. Bedankt dat je mijn paranimf wilt zijn en hopelijk zal je succesvolle carrière in Nijmegen het onderhouden van onze gezellige bijkletsdates niet in de weg staan.

Saskia, tijdens de eindfase van jouw geneeskunde opleiding was je al volop gestart met jouw onderzoek. We werden kamergenootjes in deze eerste fase van onze prille onderzoekscarrière. Ik heb je altijd bewonderd om je gedrevenheid om zo snel en efficiënt mogelijk je doel te bereiken. Jij bent zo iemand die niet afwijkt van deadlines en hebt daardoor ook je promotietraject op zeer beperkte tijd succesvol afgerond om zo snel mogelijk je vervolgopleiding tot internist aan te vatten. Ik vind het super fijn dat we nog regelmatig samen een bijkletsdinertje of -lunch (in de kliniek) plannen en hoop dat we dit zullen blijven doen. Bedankt dat je mijn paranimf wilt zijn en veel succes met je carrière als internist en met de volgende fasen in je leven samen met Leon.

\section{Het plaatjeslab}

Allereerst wil ik de centrale spil van ons lab bedanken voor alles wat ze voor mij gedaan heeft. Marion, bij jou kon ik altijd terecht voor hulp bij moeilijke protocollen, inventiviteit bij het (ver)doen van experimenten en ook goede, vaak ook diepzinnige, gesprekken. Jouw onuitputtende interesse in het menselijke gedrag heeft vaak tot interessante gespreksonderwerpen geleid. Het was ook altijd fascinerend om te zien hoe mijn vaak te gecompliceerde denkweg tot eenzelfde uitkomst leidde als jouw recht-toe-recht-aan logica. Bedankt voor het doen van een poging om mij het 'boeren'verstand bij te brengen. Ik waardeer jouw eerlijkheid en volharding. Ik wens jou al het beste in alles wat je doet, onder andere met de verdere afronding van je studie en vervolgcarrièrre.

Marijke, van jou heb ik erg veel geleerd: in vitro experimenten tijdens mijn bachelorstage, dierexperimentele proeven aan het begin van mijn promotietijd en ook het durven maken van keuzes waarbij jij kiest en je niet laat kiezen. Veel geluk en succes in je verdere carrière en met je gezin.

Reyhan, it was really great sharing a room with you and learning to know you. I admire your courage, persistence and passion for your carrier. I am very glad you could finish your $\mathrm{PhD}$ period in time and that you are now reunited with your lovely, supportive housband. Good luck with your scientific carrier in Turkey and all the best for you and your family.

Mijn andere kamergenootjes, Roger en Moniek, wil ik bedanken voor de leuke babbels en het delen van de PhD-gerelateerde ergernissen. Roger, we hebben vaker samengewerkt met succesvolle resultaten. Jouw nieuwsgierigheid en onthoudcapaciteiten van de kleinste details zijn bewonderingswaardig. Ik wens je veel succes met het afronden van je proefschrift en de spannende en leuke veranderingen binnen je privéleven die er aankomen. Moniekje, ik vond het altijd erg amusant jouw manier van discussie en het uiten van eigen mening aan te horen, voornamelijk in discussie met Roger. Als 'schuchtere' Belg heb ik daar toch enige assertiviteitstraining aan opgedaan $(-)$. Veel succes met je vervolgcarrière!

Nadine, je was mijn eerste student die ik heb begeleid voor een langere periode in ons lab. Dit was een super fijne ervaring die grotendeels te wijten is aan jouw inzet en nauwkeu- 
righeid bij het doen van de experimenten. In die tijd waren de inhoudelijke discussies tussen Johan en mij die zeer in detail gingen soms moeilijk te volgen, maar we waren ervan overtuigd dat jij als PhD-student erg veel in je mars zou hebben. Dit heb je tot nu toe ook al laten blijken. Heel veel succes met het vervolg van je promotietraject binnen de plaatjesgroep.

Ook de andere (voor mij nieuwe) AIO's en medewerkers bij de plaatjesgroep waaronder Suzanne, Frauke en Roosje wil ik veel succes en plezier wensen in hun werk

Marisa, ondanks dat ge nu niet meer echt bij de plaatjesgroep hoort, was dat tijdens mijn PhD tijd wel het geval en zal ik, naast het feit dat ge mijn carpoolina was, dat ook zo blijven herinneren. Tijdens onze gezamelijke autoritten naar de uni was het altijd gezellig bijpraten over leuke dingen en ook de werkgerelateerde en andere frustraties konden we goed bij mekaar kwijt. Bedankt voor deze fijne tijd en het geduld als ik weer eens een beetje te laat was $(-)$. Ondertussen zijt ge al enorm opgeschoten met uw promotieonderzoek en kan ik $\mathrm{u}$ al succes wensen met de afronding van deze intensieve en veeleisende periode.

Paola, ook met jou heb ik een kamer gedeeld, experimenten gedaan en buitenlandse tripjes ondernomen. Jij bent ondertussen ook niet meer echt/volledig werkzaam bij de plaatjesgroep, maar voor mijn herinnering blijft dit wel zo. Veel succes met het vervolg van je carrière.

Ook mijn dank aan alle andere biochemiemedewerkers buiten de plaatjesgroep die op een of andere wijze hebben bijgedragen aan de fijne en productieve tijd die ik er heb doorgebracht!

\section{Ondersteuning vanuit mijn opleiding}

Graag wil ik ook de ondersteuning vanuit mijn opleiding bedanken voor flexibiliteit en bemiddeling bij het afronden van mijn proefschrift in combinatie met de master tot ArtsKlinisch Onderzoeker. In het bijzonder gaat mijn dank uit naar mijn counselor, Bas Verhoeven, studieadviseur en biochemiemedewerker, Guido Tans, en mijn medestudenten (cohort 2010). De interesse en de flexibiliteit die jullie toonden was erg fijn. Het wisselen van de stageplek leverde mij extra tijd op, bedankt hiervoor Benedikt.

\section{Ontspanningsgenootjes}

Behalve de drukke combinatie van studeren, een huis en gezin hebben, en het afronden van mijn proefschrift heb ik gelukkig ook nog af en toe heerlijk kunnen genieten van etentjes, bijkletsmomenten en feestjes met Gisi, mijn Kottreesjes en de Uylenspiegels met hun madammen. Bedankt voor het begrip als ik weer eens geen tijd had, maar nog harder bedankt voor de interesse en vooral de heerlijk ontspannende momenten samen.

\section{Familie}

Een zeer groot deel van mijn dank gaat uit naar mijn zorgzame en bijspringende ouders, schoonouders en zussen. Jullie interesse en steun hebben het mede mogelijk gemaakt dat ik de nodige tijd aan mijn carrièrre heb kunnen en kan besteden. 
Ilse, de relativerende babbels met jou, onder andere over het 'ivoren torentje', hebben een gunstige invloed gehad op de keuze voor mijn vervolgcarrièrre. Bedankt om er altijd voor mij te zijn.

Mama en papa, bedankt voor alle kansen die ik gekregen heb en die ik nu nog steeds krijg. Jullie hebben mij altijd de vrije keuze gelaten, gesteund in mijn beslissingen en blijven geloven in mijn kunnen. Op alle mogelijke manieren proberen jullie mij te helpen om toch maar mijn doel te bereiken. Zonder jullie had ik dit alles nooit kunnen verwezenlijken. Heel erg bedankt voor alles!

Liefste Willem, het grootste deel van mijn dank gaat uiteraard naar u. Al tijdens mijn studie Biomedische Wetenschappen in Diepenbeek, waar we ons ook hebben leren kennen, was je er altijd voor mij. Samen met u ben ik door dat moeilijke eerste jaar geraakt. En hebt ge ervoor gezorgd dat mijn zelfvertrouwen over de jaren heen steeds sterker werd. Al het geduld dat ge bij mijn eerste thesis hebt gehad... en mij dan toch nog aanmoedigen om nog een master te gaan doen om eindelijk dokter te kunnen worden. De werkzaamheden aan ons huis waren(zijn) nog niet volledig afgerond, een nieuwe studie was aangevat en toen moest dat proefschrift ook nog af. Er zijn momenten geweest waarop ik echt heb overwogen dit laatste niet te voltooien. Het is dan ook door uw steun, geduld en hulp dat dit boekje tot stand is gekomen. En dit terwijl ge zelf ook nog enkele ambitieuze nevenactiviteiten hebt aangenomen. Duizend maal bedankt voor het zijn van zo een geweldige echtgenoot! Ik hoop dat we binnenkort samen kunnen genieten van ons langverwachte gezinnetje. 

Curriculum Vitae 
Karen Gilio was born on July 14th 1983 in Maaseik, Belgium. In 1995, she started secondary school at the Heilig-Hart College in Maasmechelen, where she received her MathematicsScience degree in 2001. Then, she studied Biomedical Sciences at the University of Hasselt in Belgium. She obtained her Master's degree in Clinical and Molecular Sciences with magna cum laude on 7th July 2006. Subsequently, she started as a PhD student at the Cardiovascular Research Institute Maastricht (CARIM) at the Department of Biochemistry (Maastricht University, the Netherlands). Under the supervision of Prof. Dr. J.W.M. Heemskerk, she performed cellular research in the field of thrombosis and haemostasis (this thesis) and contributed to several teaching activities. During this four-year period she participated in congresses in Wittenberg-Lutherstadt (Germany), Geneva (Switzerland), London (United Kingdom), Boston (United States of America), and Nuremberg (Germany), to present her research. Part of her research was performed in the laboratories of Prof. Dr. S.P. Watson (Birmingham, United Kingdom), Prof. Dr. A.W. Poole (Bristol, United Kingdom), and Prof. Dr. B. Nieswandt (Wurzburg, Germany). To become a Medical Doctor, she started in September 2010 the four-year during Master in Medicine and Clinical Science at Maastricht University. 
Karen Gilio werd geboren op 14 juli 1983 te Maaseik, in België. In 1995 begon ze haar middelbare school opleiding aan het Heilig-Hart College in Maasmechelen. Ze behaalde haar diploma in Wetenschappen-Wiskunde in 2001. Vervolgens startte ze met de opleiding Biomedische Wetenschappen aan de Universiteit van Hasselt (België), waarvoor zij met grote onderscheiding haar Master diploma behaalde op 7 juli 2006. Aansluitend begon ze als PhDstudent bij het Departement Biochemie van het Cardiovascular Research Institute Maastricht (CARIM) aan de Universiteit van Maastricht (Nederland). Onder begeleiding van Prof. Dr. J.W.M. Heemskerk, verrichtte ze cellulair onderzoek binnen het domein van trombose en hemostase (dit proefschrift), alsook toonde ze een actieve bijdrage aan verschillende onderwijsactiviteiten. Gedurende deze vierjarige periode presenteerde ze haar onderzoek op congressen in Wittenberg-Lutherstadt (Duitsland), Genève (Zwitserland), Londen (Groot-Brittannië), Boston (Verenigde Staten) en Nuremberg (Duitsland). Een gedeelte van haar onderzoek verrichtte zij in de laboratoria van Prof. Dr. S.P. Watson (Birmingham, Groot-Brittannië), Prof. Dr. A.W. Poole (Bristol, Groot-Brittannië) en Prof. Dr. B. Nieswandt (Würzburg, Duitsland). In september 2010 startte ze met de vierjarige Master opleiding tot Arts en Klinisch Onderzoeker aan de Universiteit van Maastricht. 

Publications 


\section{Papers}

1. Munnix IC, Gilio K, Siljander PR, Raynal N, Feijge MA, Hackeng TM, Deckmyn H, Smethurst PA, Farndale RW, and Heemskerk JW. Collagen-mimetic peptides mediate flow-dependent thrombus formation by high- or low-affinity binding of integrin $\alpha_{2} \beta_{1}$ and glycoprotein VI. J Thromb Haemost. 2008;6:2132-42. Impact factor: 5.439

2. Hall KJ, Harper MT, Gilio K, Cosemans JM, Heemskerk JW, and Poole AW. Genetic analysis of the role of protein kinase $\mathrm{C} \theta$ in platelet function and thrombus formation. PLoS One. 2008;3:e3277. Impact Factor: 4.411

3. Kuijpers MJ, Gilio K, Reitsma S, Nergiz-Unal R, Prinzen L, Heeneman S, Lutgens E, van Zandvoort MA, Nieswandt B, Egbrink MG, and Heemskerk JW. Complementary roles of platelets and coagulation in thrombus formation on plaques acutely ruptured by targeted ultrasound treatment: a novel intravital model. J Thromb Haemost. 2009;7:152-61. Impact factor: 5.439

4. Konopatskaya O, Gilio K, Harper MT, Zhao Y, Cosemans JM, Karim ZA, Whiteheart SW, Molkentin JD, Verkade P, Watson SP, Heemskerk JW, and Poole AW. PKC $\alpha$ regulates platelet granule secretion and thrombus formation in mice. J Clin Invest. 2009;119:399-407. Impact factor: 14.152

5. Gilio K, Munnix IC, Mangin P, Cosemans JM, Feijge MA, van der Meijden PE, Olieslagers S, Chrzanowska-Wodnicka MB, Lillian R, Schoenwaelder S, Koyasu S, Sage SO, Jackson SP, and Heemskerk JW. Non-redundant roles of phosphoinositide 3kinase isoforms $\alpha$ and $\beta$ in glycoprotein VI-induced platelet signaling and thrombus formation. J Biol Chem. 2009;284:33750-62. Impact factor: 5.328

6. Gilio K, Harper MT, Cosemans JM, Hall KJ, Konopatskaya O, Munnix IC, Molkentin $J D$, Prinzen L, Leitges $M$, Heemskerk JW, and Poole AW. Functional divergence of platelet protein kinase $C$ isoforms in thrombus formation on collagen. J Biol Chem. 2010;285:23410-9. Impact factor: 5.328

7. Gilio K, van Kruchten R, Braun A, Berna-Erro A, Feijge MA, Stegner D, van der Meijden PE, Kuijpers MJ, Varga-Szabo D, Heemskerk JW, and Nieswandt B. Roles of platelet STIM1 and Orai1 in glycoprotein VI- and thrombin-dependent procoagulant activity and thrombus formation. J Biol Chem. 2010;285:23629-38. Impact factor: 5.328

8. Konopatskaya O, Matthews SA, Harper MT, Gilio K, Cosemans JM, Williams CM, Navarro MN, Carter DA, Heemskerk JW, Leitges M, Cantrell D, and Poole AW. Protein kinase $C$ mediates platelet secretion and thrombus formation through protein kinase D2. Blood. 2011;118:416-24. Impact factor: 10.558

9. Van der Meijden PE, Feijge MA, Swieringa F, Gilio K, Nergiz-Unal R, Hamulyák K, and Heemskerk JWM. Key role of integrin $\alpha$ llb $\beta_{3}$ signaling to Syk kinase in tissue factorinduced thrombin generation. Cell Mol Life Sci. 2012;69:3481-92. Impact factor: 7.047

10. Gilio K, Mattheij N, van Kruchten R, Feijge MA, Cosemans JM, Bevers EM, and Heemskerk JW. Regulation of integrin $\alpha$ llb $\beta_{3}$ closure in procoagulant platelets. Submitted for publication 


\section{Abstracts}

1. Kuijpers MJ, Gilio K, Megens RT, Lutgens E, Heeneman S, van Zandvoort MA, oude Egbrink MG, and Heemskerk JW. Visualization of thrombus formation on acutely ruptured plaques in vivo. J Thromb Haemost 2007; 5 Supplement 2: O-T-074.

2. Munnix IC, Gilio K, Raynal N, Feijge MA, Fletcher J, Siljander PR, Farndale RW, and Heemskerk JW. Flow induced thrombus formation on immobilized collagen receptorbinding peptides. J Thromb Haemost 2007; 5 Supplement 2: P-W-259.

3. Gilio K, Harper MT, Cosemans JM, Hall KJ, Konopatskaya O, Molkentin JD, Munnix IC, Littman DR, Poole AW, and Heemskerk JW. Opposite role of platelet PKC isoforms $\alpha$, $\delta$ and $\theta$ in flow-induced thrombus formation. Proceedings of the gth UK - 1st Netherlands Platelet Meeting, London, UK 2007.

4. Kuijpers MJ, Gilio K, Megens RT, Lutgens E, Heeneman S, van Zandvoort MA, Nieswandt B, oude Egbrink MG, and Heemskerk JW. A new model of thrombus formation on acutely ruptured plaques in vivo. Proceedings of the gth UK - 1st Netherlands Platelet Meeting, London, UK 2007.

5. Hall KJ, Harper MT, Gilio K, Cosemans JM, Heemskerk JW, and Poole AW. Genetic analysis of the role of protein kinase $\mathrm{C} \theta$ in platelet function and thrombus formation. Proceedings of the 1oth UK Platelet Meeting, Bradford, UK 2008.

6. Gilio K, van Kruchten R, Braun A, Elvers M, van der Meijden PE, Feijge MA, Kuijpers MJ, Stegner D, Heemskerk JW, and Nieswandt B. Role of platelet Orai1 and Stim1 in GPVI-dependent and GPVI-independent thrombus formation and procoagulant activity. J Thromb Haemost 2009;7: Supplement 2.

7. Gilio K, Harper MT, Cosemans JM, Hall KJ, Konopatskaya O, Munnix IC, Molkentin JD, Littman DR, Heemskerk JW, and Poole AW. Divergent roles of conventional and novel PKC isoforms in platelet activation towards thrombus formation. J Thromb Haemost 2009;7: Supplement 2.

8. Schols SE, Berny MA, Cosemans JM, Gilio K, Feijge MA, Deckmyn H, and Heemskerk JW. New role of platelets in inducing fibrin formation under flow. J Thromb Haemost 2009;7: Supplement 2.

9. Van der Meijden PE, Feijge MA, Gilio K, Hamulyak K, and Heemskerk JW. Integrin $\alpha$ llb $\beta_{3}$ outside-in signaling via syk induces platelet procoagulant activity. J Thromb Haemost 2009;7: supplement 2.

10. Gilio K, Mattheij N, Feijge MA, van Kruchten R, Cosemans JM, and Heemskerk JW. Regulation of integrin closure in procoagulant platelets. Hämostaseologie 2010; 30: Ag2.

11. Van Kruchten R, Gilio K, Feijge MA, Nieswandt B, Bevers EM, and Heemskerk JW. Inhibitors of store-operated calcium entry to target the platelet procoagulant response. Hämostaseologie 2010; 30: A93.

12. Mattheij N, Gilio K, van Kruchten R, Feijge MA, Bevers EM, Jobe S, Cosemans JM, and Heemskerk JW. Regulation of integrin closure in procoagulant platelets. J Thromb Haemost 2011;7: Supplement 2. 
13. Mattheij NJ, Gilio K, van Kruchten R, Jobe SM, Du X, Wieschhaus AJ, Chishti AH, Collins $\mathrm{P}$, Heemskerk JW, and Cosemans JM. Dual mechanism of integrin closure in procoagulant platelets. EUPLAN Conference 19-21 September 2012

\section{Oral presentations and awards}

1. Student award 2005 of the Faculty of Medicine for 'best poster presentation' on the biomedical research topic "Diamond-based biosensors", University of Hasselt, Belgium.

2. Travel award 2007 from Tebu Bio Innovative Lab Services \& Reagents, the Netherlands to visit the XXIst Congress of the International Society on Thrombosis and Haemostasis (ISTH), Geneva, Switzerland.

3. Oral presentation: Opposite role of platelet PKC isoforms $\alpha, \delta$ and $\theta$ in flow-induced thrombus formation. gth UK - 1st Netherlands Platelet Meeting, 2007 - London, UK.

4. Young investigator award 2009 of the International Society on Thrombosis and Haemostasis, XXIIth Congress ISTH, Boston, USA.

5. Oral presentation: Divergent roles of conventional and novel PKC isoforms in platelet activation towards thrombus formation. XXIIth Congress of the International Society on Thrombosis and Haemostasis (ISTH), 2009 - Boston, USA. 
Addendum

Color figures chapters 4, 5, and 7 


\section{Color figures chapter 4}

A

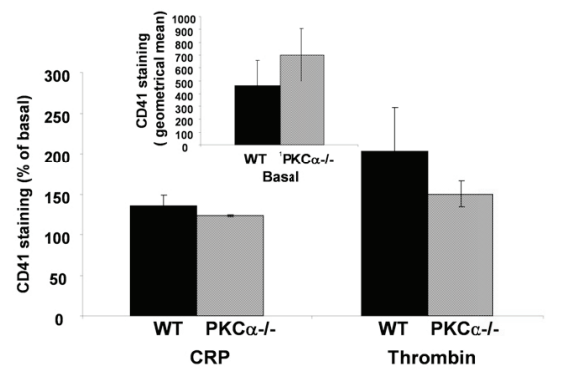

B
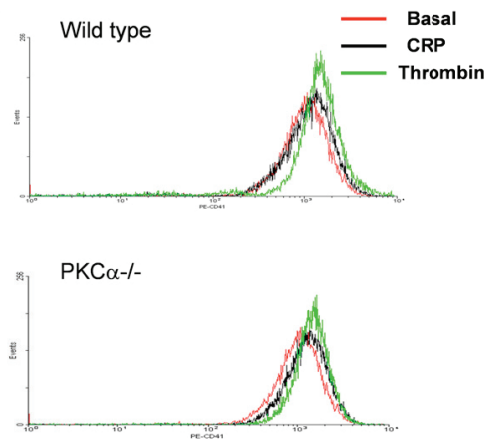

Supplementary Figure 2. Platelet surface expression of $C_{41}$ is not affected by PKC $\alpha$ knock-down. Washed platelets from wild type (WT) or Prkca ${ }^{-1-}$ mice were labelled with PE-anti-CD41 antibody, stimulated with CRP $(5 \mu \mathrm{g} / \mathrm{ml})$ or thrombin (1 unit/ml) for $15 \mathrm{~min}$ and immunofluorescence intensity measured by flow cytometry. In (A), the data presented are geometric means as percentages of basal nonstimulated levels (error bar represents SEM, $n=4$ ). Inset panell shows geometric mean of fluorescence (arbitrary fluorescence units) of basal platelets from either wild type or Prkca ${ }^{-1-}$ mice. No difference was seen between wild type and Prkca ${ }^{-/}$platelet expression of CD.41, and PKC $\alpha$ expression plays no role in regulating $C_{41}$ surface expression upon stimulation with thrombin or CRP. Data shown are mean \pm $\mathrm{SEM}, \mathrm{n}=4$. Additionally, no difference was seen between basal and CRP- or thrombin-stimulated platelets, from either wild type or Prkca ${ }^{--}$mice, in staining with an isotype-matched control antibody (PEconjugated rat IgG1; data not shown). In (B), representative frequency histograms are shown for $C_{41}$ expression from wild type (upper panel) and Prkca $^{-l_{-}}$(lower panel) platelets, either basal or stimulated with CRP $(5 \mu \mathrm{g} / \mathrm{ml})$ or thrombin ( $1 \mathrm{unit} / \mathrm{ml}$ ) for $15 \mathrm{~min}$ as indicated. Data are representative of 4 independent experiments. 
A

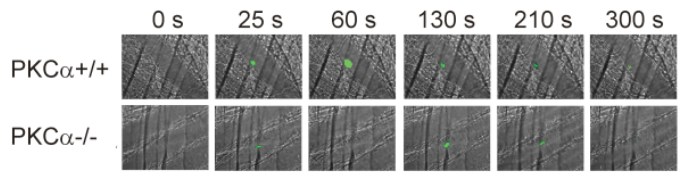

C

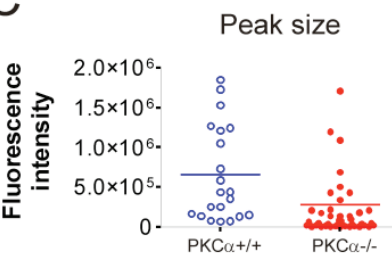

D

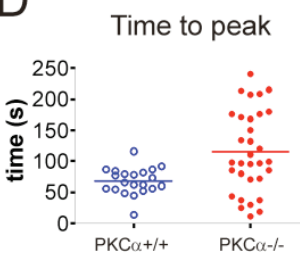

B

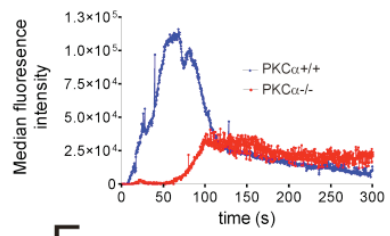

$\mathrm{E}$

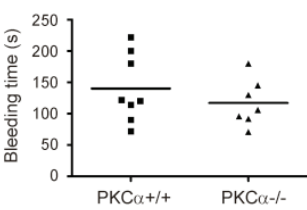

Figure 7. In vivo thrombus formation is impaired in the absence of $P K C \alpha$, but tail bleeding time is normal. (A)-(D) Mice were either Prkcal- or littermate-matched wild-type controls. Platelets were labelled in vivo with Alexa 488, as described in Methods. (A) Platelets (green) composited with brightfield images (black/white) of the cremaster arteriole were viewed at $\times 40$ magnification and images were acquired using a digital CCD camera (SensiCam II, Cooke Corporation) with a $640 \times 480$ pixel array. (B) Traces shown are median integrated platelet fluorescence of 15 thrombi induced in 3 or more mice for each group. Fluorescent intensity of platelets in arbitrary units is presented as a function of time. (C) Data are presented as a scatter diagram, and the horizontal bar is mean of 15 thrombi induced in at least 3 mice for each group. There is a significant reduction in thrombus intensity in Prkcal- by comparison with wild type controls (mean Prkca ${ }^{+/+}, 652200$; mean Prkca ${ }^{-1}, 279600 ; \mathrm{p}<0.05$ ). (D) Time to reach peak thrombus size also significantly differed from wild type controls, with mean data shown by the horizontal bars (mean Prkca ${ }^{+/+}, 68.1 \mathrm{~s} ;$ mean Prkca ${ }^{-1}, 115.2 \mathrm{~s} ; \mathrm{p}<0.01$ ). (E) Mice were anesthetized and a transverse incision made with a scalpel at a position where the diameter of the tail was 2.25 to $2.5 \mathrm{~mm}$. The tail was immersed in normal saline $\left(37^{\circ} \mathrm{C}\right)$ in a hand-held test tube. The time from incision to cessation of bleeding was recorded, and mean times are shown as horizontal bars. No significant difference was seen comparing wild type mice (mean $140 \mathrm{~s}$ ) with Prkca $^{-1 /}$ (mean $117 \mathrm{~s} ; \mathrm{p}>0.05$ ). 


\section{Color figures chapter 5}

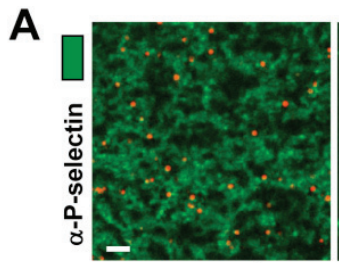

$\mathrm{PKCa}^{+/+}$

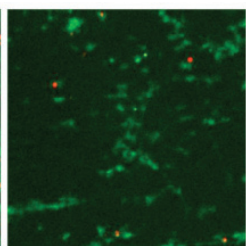

$\mathrm{PKC}^{-1-}$

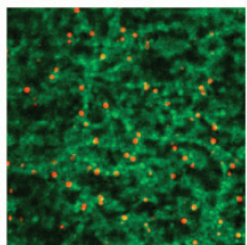

$\mathrm{PKC}^{+/+}$

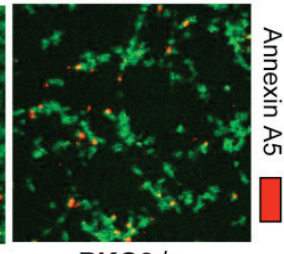

PKC $\beta^{-1-}$

B $P$-selectin
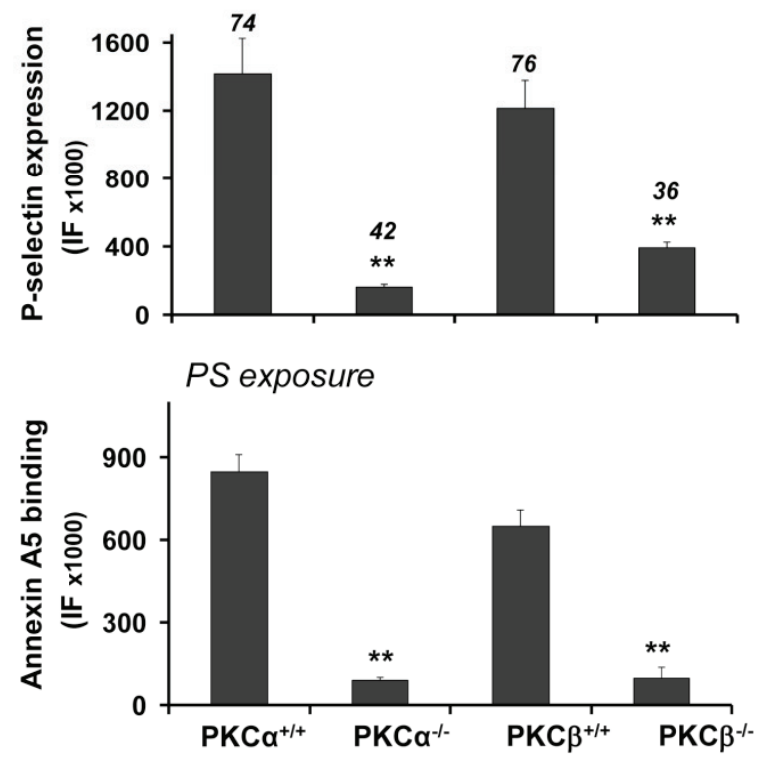

Figure 2. Deficiency of $\mathrm{PKC} \alpha$ or $\beta$ suppresses collagen-induced activation of platelets in thrombi. Whole blood from indicated mice was flowed over collagen (see Fig. 1), and platelet thrombi were double-stained with FITC-anti-mouse CD62P mAb (green) and AF647-annexin A5 (red). A, Representative confocal images $(180 \times 180 \mu \mathrm{m})$ after staining. $B$, Integrated fluorescence intensity presenting activated platelets in arbitrary units, to quantify cumulative expression of P-selectin and PS. Means \pm S.E.M. $(n=3-5) ; * * p<0.01$ vs. corresponding wildtypes. Numbers above each bar indicate the mean integrated pixel density of $\mathrm{P}$-selectin staining, an indication of staining per platelet. 


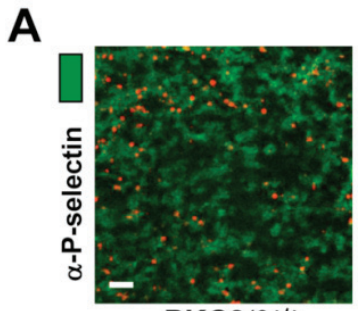

PKC $\delta / \theta^{+/+}$

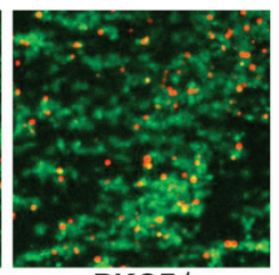

PKC ${ }^{-1-}$

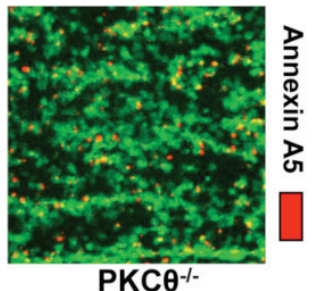

PKC ${ }^{-1-}$

B
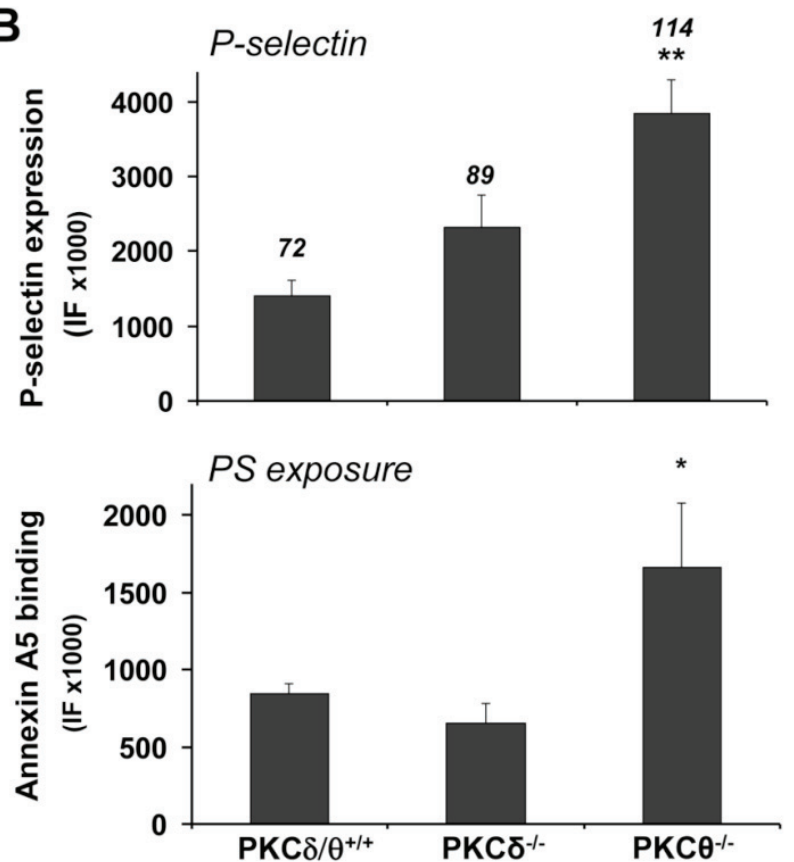

Figure 5. Deficiency of PKC $\theta$ but not $\delta$ increases collagen-induced activation of platelets in thrombi. Blood from $\mathrm{PKC} \delta^{--}, \mathrm{PKC} \theta^{-\sigma_{-}}$or matched wildtype mice was flowed over collagen, and platelet thrombi were double stained with FITC-anti-mouse CD62P mAb (green) and AF647-annexin A5 (red). A, Representative confocal images $(180 \times 180 \mu \mathrm{m})$ after staining. $B$, Integrated fluorescence intensity presenting activated platelets in arbitrary units, to quantify cumulative expression of P-selectin and PS. Means \pm S.E.M. $(n=3-5) ; * *<<0.01$ and $* p<0.05$ vs. corresponding wildtypes. Numbers above each bar indicate the mean integrated pixel density of P-selectin staining, an indication of staining per platelet. 


\section{Color figure chapter 7}

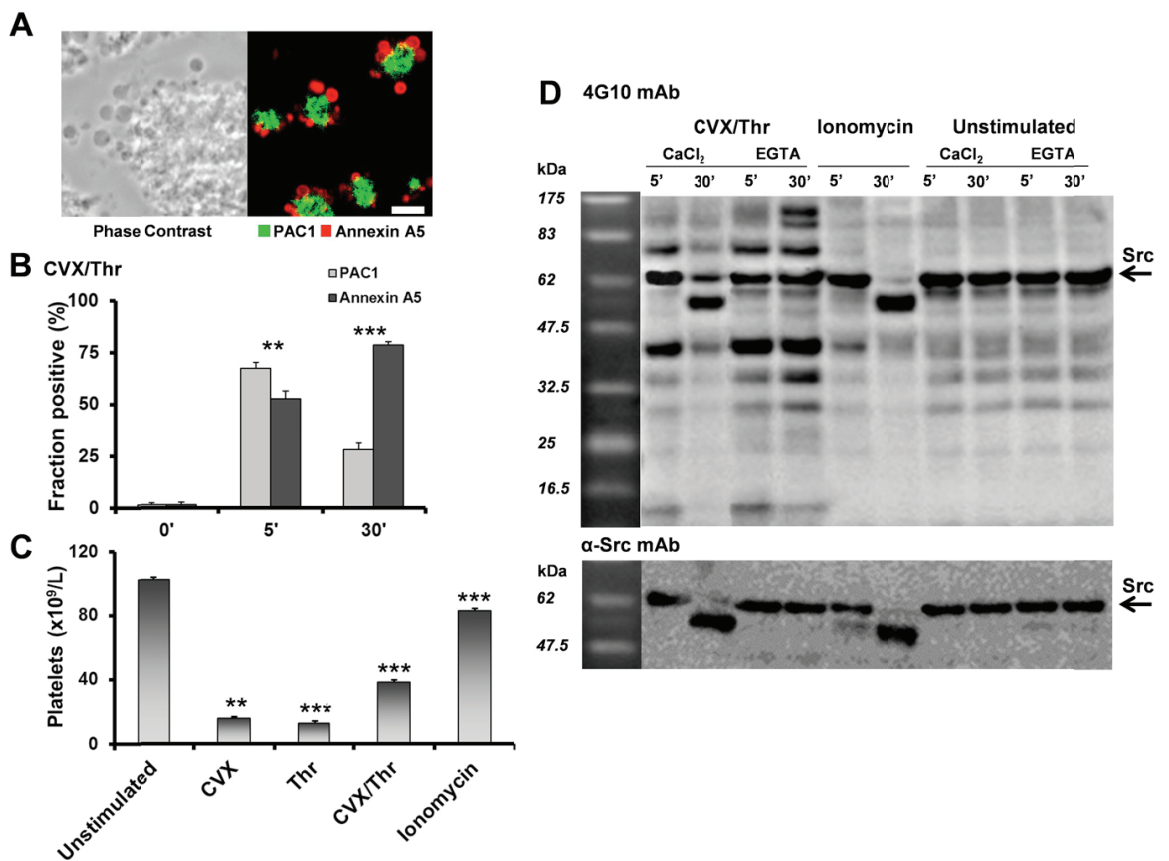

Figure 1. Integrin $\alpha$ llb $\beta_{3}$ closure in PS-exposing platelets is accompanied by Src kinase cleavage. A, Ex vivo flow experiments were performed with PPACK-anticoagulated human blood perfused over a collagen surface at $1000 \mathrm{~s}^{-1}$ for 4 minutes. Shown is a representative phase contrast image and a twocolor fluorescence image, after staining for activated integrin $\alpha \mathrm{llb} \beta_{3}$ (FITC-PAC1 mAb) and PS exposure (AF647-annexin A5); bar, $15 \mu \mathrm{m}$. B, Flow cytometric analysis of washed human platelets stimulated for 5-30 minutes with convulxin $(\mathrm{CVX}, 100 \mathrm{ng} / \mathrm{ml})$ plus thrombin $(\mathrm{Thr}, 4 \mathrm{nM})$ in the presence of $2 \mathrm{mM} \mathrm{CaCl} 2$. Shown are platelet fractions with activated $\alpha$ llb $\beta_{3}$ (FITC-PAC 1 positive) or exposed PS (FITC-annexin A5-positive). Mean \pm S.E. $(n=8-12) ; * *, p<0.02 ; * * *, p<0.001$. C, Single platelet counts after 5 minutes stimulation of platelets with CVX $(100 \mathrm{ng} / \mathrm{ml})$, $\operatorname{Thr}(4 \mathrm{nM})$, or ionomycin $(20 \mu \mathrm{M})$, in the presence of 2 $\mathrm{mM} \mathrm{CaCl}$. Mean \pm S.E. $(n=3) ; *, p<0.05$. D, Representative western blots from platelets stimulated for 5-30 minutes with CVX $(100 \mathrm{ng} / \mathrm{ml})$ and Thr $(4 \mathrm{nM})$ or ionomycin $(20 \mu \mathrm{M})$. Activations were in the presence of $2 \mathrm{mM} \mathrm{CaCl} 2$ or a $2 \mathrm{mM} \mathrm{EGTA}$, as indicated. Blots were immunostained with $4 \mathrm{G} 10 \mathrm{mAb}$ (upper), and reprobed with anti-Src Ab (lower panel). Arrows indicate position of Src family proteins. 

